


\title{
Process Modeling for the Integrated Nonthermal Treatment System (INTS) Study
}

\author{
Blaine W. Brown
}

Published April 1997

\author{
Idaho National Engineering Laboratory \\ Chemical and Environmental \\ Lockheed Martin Idaho Technologies Company \\ Idaho Falls, Idaho 83415
}

Prepared for the

U.S. Department of Energy

Under DOE Idaho Operations Office

Contract DE-AC07-94ID13223 


\section{DISClamaxi}

Portions of this dosument may be illegible in electranic image products. Images are produced from the best arailable origion docomenter 


\section{ABSTRACT}

This report describes the process modeling done in support of the Integrated Nonthermal Treatment System (INTS) study. This study was performed to supplement the Integrated Thermal Treatment System (ITTS) study and comprises five conceptual treatment systems that treat DOE contract-handled mixed lowlevel wastes (MLLW) at temperatures of less than $350^{\circ} \mathrm{F}$. ASPEN PLUS, a chemical process simulator, was used to model the systems. Nonthermal treatment systems were developed as part of the INTS study and include sufficient processing steps to treat the entire inventory of MLLW. The final result of the modeling is a process flowsheet with a detailed mass and energy balance. In contrast to the ITTS study, which modeled only the main treatment system, the INTS study modeled each of the various processing steps with ASPEN PLUS, release 9.3-1. Trace constituents, such as radionuclides and minor pollutant species, were not included in the calculations.

\section{DISCLAIMER}

\footnotetext{
This report was prepared as an account of work sponsored by an agency of the United States Government. Neither the United States Government nor any agency thereof, nor any of their employees, makes any warranty, express or implied, or assumes any legal liability or responsibility for the accuracy, completeness, or usefulness of any information, apparatus, product, or process disclosed, or represents that its use would not infringe privately owned rights. Reference herein to any specific commercial product, process, or service by trade name, trademark, manufacturer, or otherwise does not necessarily constitute or imply its endorsement, recommendation, or favoring by the United States Government or any agency thereof. The views and opinions of authors expressed herein do not necessarily state or reflect those of the United States Government or any agency thereof.
} 


\section{CONTENTS}

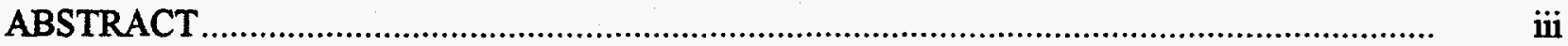

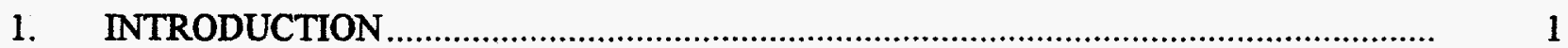

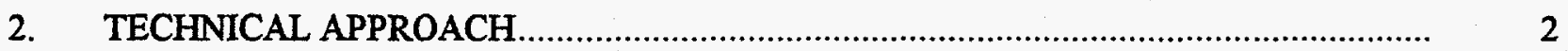

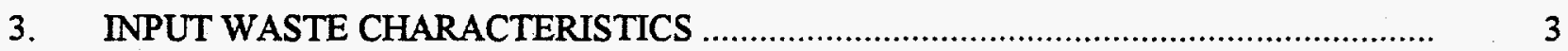

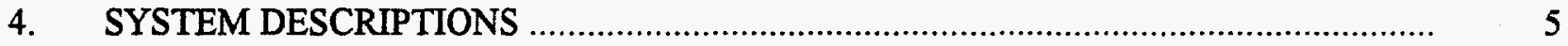

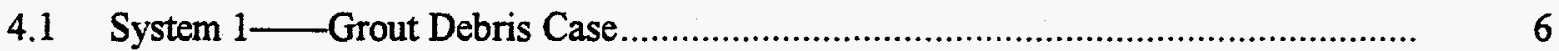

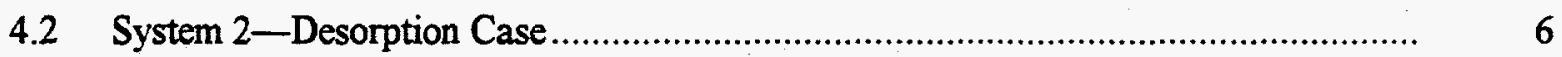

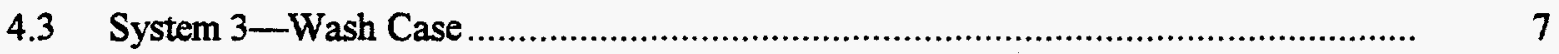

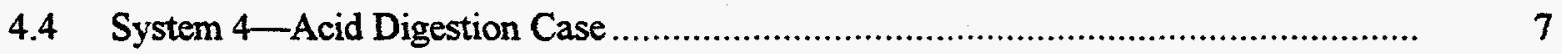

4.5 System 5-Catalytic Wet Oxidation Case ................................................. 8

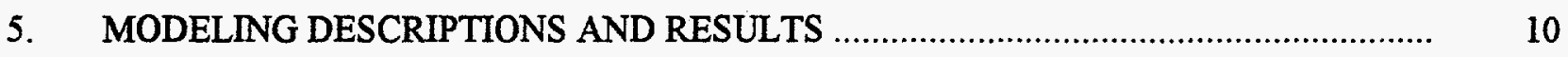

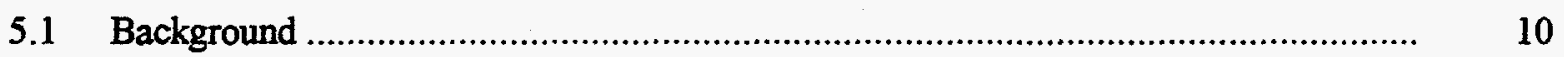

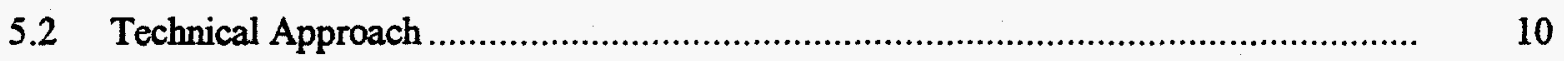

6. OVERALL MASS AND ENERGY BALANCES ....................................................... 12

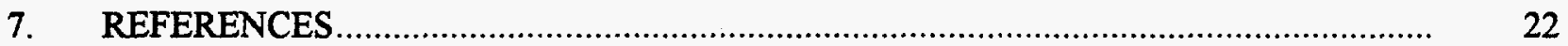

\section{TABLES}

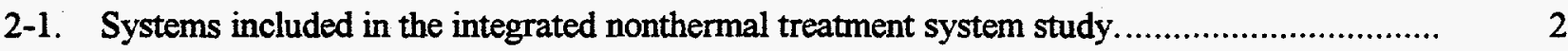

3-1. Input waste categories for the integrated nonthermal treatment system study....................... 3

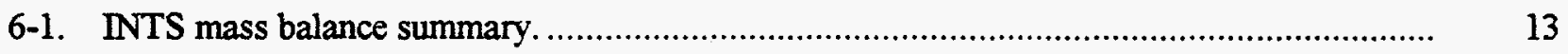

$6-2$. Integrated nonthermal treatment system study energy requirements............................... 21 


\section{Process Modeling for the Integrated Nonthermal Treatment System (INTS) Study}

\section{INTRODUCTION}

This report summarizes mass and energy balance calculations made for conceptual treatment systems analyzed by the Integrated Nonthermal Treatment System (INTS) study. The INTS study was a follow-on to the Integrated Thermal Treatment System (ITTS) study, wherein 19 different conceptual thermal treatment systems were analyzed for applicability to contact-handled mixed low-level radioactive waste (MLLW). The ITTS study Technical Support Group (TSG) reviewed the conclusions of the ITTS study and recommended that, to investigate all methods of treating the MLLW, some systems incorporating nonthermal treatment methods should also be analyzed. This report documents the process modeling only; neither process selection nor technological risks are discussed. More detailed descriptions of these issues are contained in the final report for the INTS study (Biagi et al., 1997). 


\section{TECHNICAL APPROACH}

Nonthermal methods for waste treatment are perceived by many to offer advantages over thermal methods in many areas, including safety, environmental emissions, and cost. The INTS study used the same analysis techniques as used in the ITTS study. For the sake of brevity, these techniques are not repeated here, but may be obtained in the respective references.

The ITTS study analyzed a total of 19 different systems; however, because nonthermal methods are not as mature as thermal methods for waste destruction, it was difficult to identify this many nonthermal systems for analysis. Consequently, only five nonthermal systems were analyzed. These are summarized in Table 2-1 and will be described in Section 4. Detailed descriptions are found in the INTS final report.

Table 2-1. Systems included in the integrated nonthermal treatment system study.

Grout Debris Case - mediated electrochemical oxidation of organic liquids and sludges; vacuum thermal desorption of process residue, inorganic sludges, and soil; grout stabilization of untreated debris and residual solids; polymer stabilization of salts and residual sludges

Desorption Case - catalytic wet oxidation of organic liquids and sludges; vacuum thermal desorption of process residue, inorganic sludges, debris, and soil; grout stabilization of residual solids; polymer stabilization of salts and residual sludges

Wash Case - mediated electrochemical oxidation of organic liquids and sludges; aqueous wash of process residue, inorganic sludges and soil; pressure wash of debris; grout stabilization of residual solids; polymer stabilization of residual sludges and salts

Acid Digestion Case - phosphoric/nitric acid digestion of organic liquids and sludges and soft debris; vacuum thermal desorption of process residue and inorganic sludges; aqueous wash of soil; high pressure wash of open debris; grout stabilization of complex debris; phosphate bonded ceramic stabilization of residual solids and salts

Catalytic Wet Oxidation Case - catalytic wet oxidation of organic liquids and sludges and soft debris; vacuum thermal desorption of process residue and inorganic sludges; aqueous wash of soil; high pressure wash of open debris; grout stabilization of complex debris and residual solids; polymer stabilization of salts and residual sludges 


\section{INPUT WASTE CHARACTERISTICS}

As with the ITTS study, the INTS study used a waste stream that was characteristic of all MLLW in the DOE system. The waste stream considered was identical to that considered in the ITTS study and the input feed rate of waste to the system ${ }^{1}$ was therefore the same as in the ITTS study. In the ITTS study, two thirds of the waste was treated by a single thermal process, such as incineration. For the INTS wastes, a variety of processes was required to treat the same waste. Thus in the INTS the wastes were categorized differently than in the ITTS study. This change in waste categories was one recommendation of the TSG. The waste categories used in the INTS study are shown in Table 3-1.

Table 3-1. Input waste categories for the integrated nonthermal treatment system study.

\section{Feed Rate}

Waste Category Designation $(\mathrm{lb} / \mathrm{h}) \quad$ Waste Description

\begin{tabular}{|c|c|c|c|}
\hline Aqueous waste & $\cdot 1000$ & 60.8 & Water, heavy metals \\
\hline Organic liquids, sludges & $2000 / 3000$ & 185.4 & $\begin{array}{l}\text { Organic sludge, oil, organic liquids, aqueous } \\
\text { organics }\end{array}$ \\
\hline $\begin{array}{l}\text { Process residue, } \\
\text { inorganic sludges }\end{array}$ & 3000 & 1007.2 & $\begin{array}{l}\text { Inorganic sludge, cemented organic and inorganic } \\
\text { sludge, absorbed liquids, concrete }\end{array}$ \\
\hline Salt waste & 3000 & 6.6 & Halide, nitrate, and sulfate salts \\
\hline Bulk Soil & 4000 & 328.5 & Soil/dirt \\
\hline Soft debris & 5000 & 434.8 & Paper, plastic, FRP boxes, cloth, cardboard \\
\hline Complex debris & 5000 & 177.4 & Metal drums, bulk metals \\
\hline Open debris & 5000 & 80.4 & Misc. glass \\
\hline Bulk metal & 5000 & 462.1 & Bulk nontoxic metals, metal drums \\
\hline Special waste & 6000 & 154.9 & $\mathrm{U}$ and $\mathrm{Be}$ compounds, labpacks, batteries, etc. \\
\hline Mercury Waste & 7000 & 2.5 & Bulk mercury \\
\hline Lead waste & 7000 & 26 & Lead shielding, leaded rubber \\
\hline Total & & 2927 & \\
\hline
\end{tabular}

\footnotetext{
${ }^{1}$ The Basis for the ITTS study was a nominal $2000 \mathrm{lb} / \mathrm{h}$ waste throughput in the main thermal treatment subsystem. The amount of waste that necessarily accompanies this waste is as follows: metallic waste $-149 \mathrm{lb} / \mathrm{h}$; metal to decontamination $468 \mathrm{lb} / \mathrm{h}$; lead waste $-26 \mathrm{lb} / \mathrm{h}$; mercury waste $-50 \mathrm{lb} / \mathrm{h}$; aqueous waste $-80 \mathrm{lb} / \mathrm{h}$; special wastes - $153 \mathrm{lb} / \mathrm{h}$; and halide saits $1 \mathrm{lb} / \mathrm{h}$, for a total of $2927 \mathrm{lb} / \mathrm{h}$ treated by the system.
} 
More detailed information concerning each of these waste streams can be found in INTS final report. In order to perform mass balance calculations it was necessary to compile detailed waste input tables for each of the waste categories. 


\section{SYSTEM DESCRIPTIONS}

This section provides brief descriptions of the five processing systems. More detailed descriptions of these systems and process flow diagrams (PFDs) are available in the INTS final report. It should be noted that the waste treatment technologies investigated in the INTS study are less technologically mature than the technologies investigated in the ITTS study. Consequently, the systems in the INTS study may undergo some refinement as further developments are made in nonthermal treatment technologies. The system descriptions provided herein can be only as descriptive of the state-of-the-art as it is understood at the time of the INTS study.

Certain subsystems are common to all of the processing systems considered in the INTS study. These subsystems are described below.

Receiving and Preparation. All INTS systems would necessarily incorporate a subsystem to receive waste and this subsystem would be the same for all of the systems in the INTS study. Because the treatment technologies in the INTS study can usually treat only specific wastes, receiving and preparation would be especially important for nonthermal systems. The MLLW would need to be sorted into numerous waste categories for treatment by the different nonthermal treatment technologies contained within the various INTS systems. Receiving and preparation includes unloading of the waste from storage containers, radioactive assay of the incoming waste, remote sorting, and, where appropriate, size reduction and shredding.

Aqueous Waste Treatment Subsystem. This subsystem would be identical for any of the systems in the INTS study. Aqueous waste treatment includes acid neutralization, removal of immiscible organics, precipitation of metals and concentration (evaporation) of scrubber bottoms. Photolytic UV oxidation is used for destruction of organics. Hydrogen peroxide $\left(\mathrm{H}_{2} \mathrm{O}_{2}\right)$ and ozone $\left(\mathrm{O}_{3}\right.$ are converted to hydoxyl radicals $(\mathrm{OH} \cdot)$ that combine with the organics to form $\mathrm{CO}_{2}$ and $\mathrm{H}_{2} \mathrm{O}$.

Metal Decontamination Subsystem. Bulk surface contaminated metals are decontaminated by abrasive blasting. Water and contaminants removed from the metal are sent for aqueous treatment; decontaminated metal is sent for certification and possible recycle within the DOE complex.

Special Waste Treatment Subsystem. As with the ITTS study, a certain portion of the waste is expected to be so diverse as to require treatment by methods that have yet to be identified. The treatment method or methods to be used in the special waste treatment subsystem will be defined as processes become available to treat these waste streams.

Mercury Amalgamation Subsystem. The bulk (elemental) mercury in the MLLW and mercury that can be separated from the rest of the MLLW is treated by amalgamation, wherein a stable copper-mercury amalgam is formed.

Lead Recovery Subsystem. The lead recovery subsystem separates different lead waste types. Lead brick or sheet is decontaminated by abrasive blasting. Lead with entrained contamination, such as leaded rubber gloves and similar leaded materials, is stabilized in grout.

Air Pollution Control Subsystem. Offgas from all subsystems is treated by the air pollution control subsystem. The gas is filtered to remove solids and fines and then is treated by a gas phase corona reactor which uses high voltage to destroy dilute gas-phase organics. The offgas is scrubbed with a caustic 
solution and the scrubber solution sent to aqueous waste treatment. Mist is eliminated from the offgas which is filtered and released.

\subsection{System 1-Grout Debris Case}

Organic destruction subsystem. In System 1, mediated electrochemical oxidation (MEO) is used for organic destruction. The MEO process combines a dilute stream of organic liquid waste in an electrolyte (the anolyte) containing $\mathrm{Ag}^{2+}$ ions and nitric acid (HNO3). The $\mathrm{Ag}^{2+}$ ions destroy the organic compounds by oxidation and in the process the silver is reduced to $\mathrm{Ag}^{+}$. As the electrolyte passes through an electrolytic cell, the $\mathrm{Ag}^{2+}$ ions are regenerated at the anode to continue the destruction of organic compounds. Carbon dioxide is produced from organics in the anolyte, while water is produced from oxidation of hydrogen ions $\left(\mathrm{H}^{+}\right)$at the cathode. Silver is recycled from the salt $(\mathrm{AgCl})$ that results from the process and nitric acid is recycled from the nitrous acid ( $\mathrm{HNO} 2)$ byproduct. The sodium chloride $(\mathrm{NaCl})$ solution from silver recovery is sent to the aqueous waste treatment subsystem. Offgas that results from organic destruction and nitric acid recovery is treated by the air pollution control subsystem.

Process residue and inorganic sludge treatment subsystem. In System 1, process residue and inorganic sludge is treated by a vacuum desorption process which subjects the waste to a high vacuum (1 psia) and temperatures of approximately $550^{\circ} \mathrm{F}$. The volatile material driven off is condensed; the water fraction is sent to aqueous waste treatment and the organic phase is sent to organic destruction. Noncondensible gases are treated by the offgas treatment subsystem. The solid residue is treated by a mercury leaching process and then treated by the polymer stabilization subsystem.

Bulk soil treatment subsystem. System 1 treats bulk soils by vacuum desorption described above.

Polymer stabilization subsystem. In System 1, polymer stabilization is used to treat the solid residue from vacuum desorption of process residue and inorganic sludge, salt waste in the MLLW, and salt sludge that results from the aqueous waste treatment subsystem. The incoming waste is dried to $5 \%$ moisture content, and then combined with a 1:1 ratio of polyethylene in a stabilization process. Recovered water is sent to aqueous waste treatment.

Grout stabilization subsystem. Grout stabilization, the combination of cement and waste in a 2:1 ratio, is used in System 1 as direct treatment for soft, open, and complex debris. Bulk soils, after treatment by vacuum desorption, and lead with entrained contamination are also treated by grout stabilization.

\subsection{System 2-Desorption Case}

System 2 is very similar to System 1, except for the treatment of organic liquids and sludges and soft, open, and complex debris.

Organic destruction subsystem. In System 2, catalytic wet oxidation is used for oxidation of the organic liquid and sludges. Catalytic wet oxidation uses $\mathrm{Fe}^{3+}$ in a catalyzed acid solution $\left(60 \% \mathrm{FeCl}_{3}\right.$ and $3.6 \% \mathrm{HCl}$ in a water solution with $0.001 \mathrm{M}$ of catalysts) for oxidation and destruction of organics.

Cxygen is used to regenerate $\mathrm{Fe}^{3+}$ from the $\mathrm{Fe}^{2+}$ produced in the organic oxidation reaction. The slurry from the reactor is filtered; the acid solution is recycled to the reactor and the solids are neutralized by the addition of caustic. Catalytic wet oxidation of organics results in offgas, salt solution, and solid residue, which are sent for treatment by the air pollution control, aqueous waste treatment, and polymer stabilization subsystems, respectively. The reactor operates at $301^{\circ} \mathrm{F}$ and $20 \mathrm{psig}$. 
Soft, open, and complex debris treatment subsystem. System 2 treats soft, open, and complex debris by vacuum desorption which is described above. Soft debris is treated slightly differently; vacuum desorption of soft debris is performed at $250^{\circ} \mathrm{F}$ to allow desorption of the organics and to avoid melting of the plastic waste matrix itself.

\subsection{System 3-Wash Case}

Organic destruction subsystem. System 3 treats organic liquids and sludges with MEO which is described above for System 1.

Process residue and inorganic sludge treatment subsystem. In System 3, process residue and inorganic sludge are treated by an aqueous wash process, which washes organics from the waste with a solution of water and surfactant. Caustic $(\mathrm{NaOH})$ is added to the wash water to neutralize acids, such as chlorides. The precipitate is filtered and then sent for treatment by polymer stabilization. Organics washed from the waste are separated from the wash water and sent to the organic destruction subsystem and the solid matrix is treated by polymer stabilization.

Bulk soil treatment subsystem. System 3 treats bulk soils by aqueous wash described above. Washed soil is dried and then sent to the grout stabilization subsystem. Moisture removed from the soil is treated by the aqueous waste treatment subsystem.

Soft debris treatment subsystem. Soft debris in System 3 is treated by low-pressure wash. Modeling of low-pressure wash is similar to modeling of aqueous wash. Washed debris is sent to the grout stabilization subsystem. The wash water with the washed organics is treated by the organic destruction subsystem. above.

Open debris treatment subsystem. Open debris is also treated by high pressure wash discussed

Complex debris treatment subsystem. Complex debris is also treated by low-pressure wash discussed above.

Polymer stabilization subsystem. Polymer stabilization is used to treat the solid residue from aqueous wash of process residue and inorganic sludge, salt waste in the MLLW, and salt sludge that results from the aqueous waste treatment subsystem.

Grout stabilization subsystem. Grout stabilization, the combination of cement and waste in a 2:1 ratio, is used in System 3 to treat the solid residue from washing of bulk soils, lead with entrained contamination, and soft, open, and complex debris.

\subsection{System 4-Acid Digestion Case}

Organic destruction subsystem. System 4 treats organic liquids and sludges with an acid digestion process, which uses a combination of phosphoric and nitric acid at $392^{\circ} \mathrm{F}$ and 14.7 psia to oxidize organics. The phosphoric acid serves mainly to keep the nitric acid from evaporating; the oxidation of the organics is due to the action of the nitric acid. Because phosphoric acid is not consumed by the reaction, it was not necessary to include it in the mass balance modeling. Oxygen is added to convert the nitrous acid byproduct $\left(\mathrm{HNO}_{2}\right)$ back to nitric acid which is recycled to the process. The offgas is neutralized with 
caustic $(\mathrm{NaOH})$ and the treated offgas, liquid, and solid residue are sent for treatment by the air pollution control, aqueous waste treatment, and phosphate-bonded ceramic stabilization subsystems, respectively.

Process residue and inorganic sludge treatment subsystem. In System 4, process residue and inorganic sludge is treated by vacuum desorption process, the same process used in Systems 1 and 2 and described above. Solid residue is treated by the phosphate-bonded ceramic stabilization subsystem.

Bulk soil treatment subsystem. System 4 treats bulk soils by aqueous wash, the same treatment used in System 3 and described above. The dried soil is treated by the phosphate-bonded ceramic stabilization subsystem. above.

Soft debris treatment subsystem. Soft debris in System 4 is treated by acid digestion described

Open debris treatment subsystem. Open debris is treated by high pressure wash, the same treatment used in System 3 and discussed above.

Complex debris treatment subsystem. Complex debris in System 4 is sent directly to grout stabilization, the same treatment used in System 1 and described above.

Phosphate-bonded ceramic stabilization subsystem. In System 4, solid residue from acid digestion of organic liquids and sludges and soft debris, vacuum desorption of process residue and inorganic sludge, aqueous wash of bulk soil, and salt sludge that results from the aqueous waste treatment subsystem is treated by phosphate-bonded ceramic stabilization. This process gives a high strength, low porosity solid that is an alternative to polymer stabilization.

Polymer stabilization subsystem. Polymer stabilization is used to treat the soluble salt waste in the MLLW.

Grout stabilization subsystem. Grout stabilization is used to treat the solid residue from washing of open debris, lead with entrained contamination, and complex debris.

\subsection{System 5-Catalytic Wet Oxidation Case}

Organic destruction subsystem. System 5 treats organic liquids and sludges by catalytic wet oxidation, the same process as is used in System 2. Catalytic wet oxidation uses $\mathrm{Fe}^{3+}$ in a catalyzed acid solution $\left(60 \% \mathrm{FeCl}_{3}\right.$ and $3.6 \% \mathrm{HCl}$ in a water solution with $0.001 \mathrm{M}$ of catalysts) for destruction of organics. Oxygen is added to regenerate $\mathrm{Fe}^{3+}$ from the $\mathrm{Fe}^{2+}$ byproduct. Caustic is added to neutralize acids; the precipitate is filtered from the liquid. The resulting offgas, salt solution, and solid residue are sent for treatment by the air pollution control, aqueous waste treatment, and polymer stabilization subsystems, respectively.

Process residue and inorganic sludge treatment subsystem. In System 5, process residue and inorganic sludge is treated by vacuum desorption process, the same process used in Systems 1, 2, and 4 and described above. Solid residue is treated by the polymer stabilization subsystem. 
Bulk soil treatment subsystem. System 5 treats bulk soils by aqueous wash, the same treatment used in Systems 3 and 4 and described above. The dried soil is treated by the grout stabilization subsystem.

Soft debris treatment subsystem. Soft debris in System 5 is treated by catalytic wet oxidation described above. Solid residue from this subsystem is sent to grout stabilization.

Open debris treatment subsystem. Open debris is treated by high pressure wash, the same as is Systems 3 and 4 discussed above.

Complex debris treatment subsystem. Complex debris in System 5 is sent directly to grout stabilization, the same treatment used in Systems 1 and 4 and described above.

Polymer stabilization subsystem. In System 5, polymer stabilization is used for treatment of solid residue from catalytic wet oxidation of organic liquids and sludges, vacuum desorption of process residue and inorganic sludge, salt waste, and salt sludge that results from the aqueous waste treatment subsystem.

Grout stabilization subsystem. Grout stabilization is used to treat the solid residue from catalytic wet oxidation of soft debris, washing of soil and open debris, lead with entrained contamination, and complex debris. 


\section{MODELING DESCRIPTIONS AND RESULTS}

\subsection{Background}

This work is an extension of the modeling that was performed for the 19 systems of the ITTS study. For mass and energy balance calculations, the database of MLLW used herein was the same as that used in the ITTS study. However, these wastes were categorized slightly differently for this study as mentioned in Section 3.

ASPEN PLUS was used to model the INTS systems. ASPEN PLUS is a chemical process simulator that allows mass and energy balances to be obtained from user-defined process flow diagrams. The code can model most unit operations in chemical engineering, such as chemical reactors, flow splitters, separators, mixers, distillation columns, etc. In addition, ASPEN PLUS allows for detailed feedback loops and design specifications that enable the user to specify certain design conditions and have the appropriate reaction conditions or feed requirements calculated by the code. Appendix B includes the ASCII input files for the ASPEN PLUS models for the INTS study. More details concerning ASPEN PLUS are found in the ITTS modeling report (Liebelt et al., 1995).

\subsection{Technical Approach}

As with the ITTS study, the objective of the ASPEN PLUS modeling was to achieve a mass balance for each system and to determine the energy requirements for treating the waste. These results would be used to make estimates on the life-cycle costs of MLLW treatment and the effluent from each system.

Because only five different systems were considered in the INTS study, compared to 19 systems in the ITTS study, it was decided to model each subsystem in the INTS study. ASPEN PLUS calculations for the ITTS study did not consider all of the subsystems because of the large number of systems being considered. In addition, modeling of the individual subsystems was necessary in the INTS study because they were more diverse than the subsystems of the ITTS study and the results from the mass balances would have more of an impact on the total system mass balance.

The special waste treatment subsystem was included in the systems modeled for the INTS study. However, consistent with the ITTS study, no calculations were performed on the special waste because its treatment has not been specified.

Flow diagrams generated by ASPEN PLUS for each of the five INTS models are included in Appendix A. These diagrams correspond to the respective PFDs for the INTS study (Biagi et al., 1996). The code had some restrictions that prevented the model from corresponding exactly to the PFDs. For example, ASPEN PLUS allows only one product stream from a reactor, which means that a chemical reactor with several output streams in the PFD must be modeled by two ASPEN PLUS blocks: a chemical reactor and a separator. The complexity of the systems being modeled also necessitated the use of simplifying assumptions to keep the calculation scheme manageable. Recycling of streams within a subsystem was often not included, particularly of aqueous waste. The overall balance remains correct, but more waste is sent to aqueous treatment than is shown in the PFDs. Modeling of the MEO subsystem also combines several operations that are separate in the PFDs, such as combining the anolyte and catholyte offgas streams. In some cases, it was possible to combine several unit operations within a single block, such as the leaching of solid waste with water. 
The mass balance summary table (Table 6-1; found in Section 6) summarizes the calculations for the five INTS models. As with the ITTS study, it was not deemed necessary to provide comprehensive tables of all streams considered in the ASPEN PLUS models. The detailed stream data were used to compile the summary tables. An abbreviated form of the comprehensive stream data (including major waste constituents such as organics, water, solids and gases, as well as data for each chemical species considered) will be provided in a separate document as a supplement to the PFDs. 


\section{OVERALL MASS AND ENERGY BALANCES}

Detailed results from the ASPEN PLUS modeling were output to a spreadsheet file (Lotus 1-2-3 format). Because this table was much more comprehensive than was necessary for evaluating the mass balance, total mass flow rates from this file were linked to another spreadsheet file (Excel format). The Excel file performed all of the necessary mass balance calculations. Results of the calculations are shown in Table 6-1.

Because the entire mass balance is performed by ASPEN PLUS, the closure on the mass balance is the same as that specified in the model input. This tolerance is usually 0.0001 or tighter. The INTS models included flow rates of approximately $10,000 \mathrm{lb} / \mathrm{hr}$, which results in the mass balance closing to within $1 \mathrm{lb}$.

Also included in Table 6-1 are calculations to determine the amount of mass reduction and volume reduction that the wastes might undergo if processed by these systems. The mass reduction for the INTS systems (mass waste input/mass solids output) ranges from approximately 0.61 to 0.78 . Thus, the mass of solid material that leaves the processing systems exceeds the mass of waste treated. The volume reduction ratio (volume waste input/volume solids output), which ranges from 1.05 to 1.24 , indicates that processing of the waste results in a smaller volume of solids to be disposed. This apparent dichotomy (a larger processed mass, but a smaller processed volume) is acceptable when it is understood that the density of the solidified waste is much greater than that of the unprocessed waste.

Calculated energy requirements of the systems INTS study are seen in Table 6-2. Electrical and thermal energy requirements are converted to energy costs using an assumption of $\$ 0.05 / \mathrm{kWh}$ and $\$ 2.00 / \mathrm{MMBtu}$. Energy costs range from about $\$ 2$ per hour to over $\$ 21$ per hour, which is less than $1 \%$ of the total treatment costs. 
Table 6.1

INTS Mass Balance Summary

\begin{tabular}{|c|c|c|c|c|c|c|}
\hline & A & C & D & $E$ & $\mathbf{F}$ & $\mathbf{G}$ \\
\hline \multicolumn{7}{|l|}{1} \\
\hline 2 & System & System 1 & System 2 & System 3 & System 4 & System 5 \\
\hline 3 & & Grout Debris & Desorption & Wash I & Acid Digestion & CWO \\
\hline 4 & 稵 & 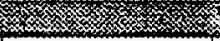 & \multicolumn{2}{|c|}{ WAST SNRYU } & 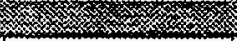 & - \\
\hline 5 & Aqueous Waste $\quad(1000)$ & 60.8 & 60.8 & 60.8 & 60.8 & 60.8 \\
\hline 6 & Organic Liquids, Sludges $(2000 / 3000)$ & 185.4 & 185.4 & 185.4 & 185.4 & 185.4 \\
\hline 7 & Residue, Inorg. Sludge $\quad(3000)$ & 1007.2 & 1007.2 & 1007.2 & 1007.2 & 1007.2 \\
\hline 8 & Salt Waste & 6.6 & 6.6 & 6.6 & 6.6 & 6.6 \\
\hline 9 & Bulk Soils & 328.5 & 328.5 & 328.5 & 328.5 & 328.5 \\
\hline 10 & Debris - Soft & 434.8 & 434.8 & 434.8 & 434.8 & 434.8 \\
\hline 11 & Debris - Complex $\quad(5000)$ & 177.4 & 177.4 & 177.4 & 177.4 & 177.4 \\
\hline 12 & Debris - Open $\quad(5000)$ & 80.4 & 80.4 & 80.4 & 80.4 & 80.4 \\
\hline 13 & Bulk Metal & 462.1 & 462.1 & 462.1 & 462.1 & 462.1 \\
\hline 14 & Special & 154.9 & 154.9 & 154.9 & 154.9 & 154.9 \\
\hline 15 & Mercury waste $\quad(7000)$ & 2.5 & 2.5 & 2.5 & 2.5 & 2.5 \\
\hline 16 & Lead Waste & 26.0 & 26.0 & 26.0 & 26.0 & 26.0 \\
\hline 17 & Total Input Waste & 2926.6 & 2926.6 & 2926.6 & 2926.6 & 2926.6 \\
\hline 18 & & & & & & \\
\hline 19 & & 28 & PROCESSINF & PUTS & & \\
\hline 20 & & & & & & \\
\hline 21 & Hg Amalgamation Subsystem & & & & & \\
\hline 22 & Waste Input & & & & & \\
\hline 23 & Mercury waste & 2.5 & 2.5 & 2.5 & 2.5 & 2.5 \\
\hline 24 & Process Inputs & & & & & \\
\hline 25 & \begin{tabular}{|c|} 
additives (copper) \\
\end{tabular} & 1.9 & 3.6 & 1.8 & 1.8 & 1.9 \\
\hline 26 & Input from other subsystems & & & & & \\
\hline 27 & process residue/inorganic sludge & 0.1 & 0.1 & & 0.1 & 0.1 \\
\hline 28 & debris treatment & & 2.5 & & & \\
\hline 29 & Total Subsystem Input & 4.5 & 8.8 & 4.3 & 4.5 & 4.5 \\
\hline 30 & & & & & & \\
\hline 31 & & & & & & \\
\hline 32 & Air Pollution Control Subsystem & & & & & \\
\hline 33 & Waste Input & & & & & \\
\hline 34 & Process Inputs & & & & & \\
\hline 35 & Air: 02 & 0.0 & 0.0 & 0.0 & 10.6 & 0.0 \\
\hline 36 & $\mathrm{N2}$ & 0.0 & 0.0 & 0.0 & 34.8 & 0.0 \\
\hline 37 & $\mathrm{NaOH}$ & 0.3 & 1.1 & 2.5 & 0.0 & 2.9 \\
\hline 38 & $\mathrm{H} 2 \mathrm{O}$ & 0.3 & 1.1 & 2.5 & 0.0 & 2.9 \\
\hline 39 & Input from other subsystems & & & & & \\
\hline 40 & aqueous waste treatment & 8.8 & 9.1 & 27.1 & 9.0 & 9.5 \\
\hline 41 & organic destruction - offgases & 580.5 & 652.6 & 799.8 & 542.4 & 561.6 \\
\hline 42 & process residue/inorganic sludge & 79.9 & 79.9 & & 79.9 & 79.9 \\
\hline 43 & debris treatment & & 55.2 & & 764.8 & 781.8 \\
\hline 44 & bulk soil treatment & 26.1 & 26.1 & & 0.0 & 0.0 \\
\hline 45 & & & & & & \\
\hline 46 & Total Subsystem Input & 695.8 & 825.0 & 831.9 & 1441.5 & 1438.5 \\
\hline 47 & & & & & & \\
\hline
\end{tabular}


Table 6.1

INTS Mass Balance Summary

\begin{tabular}{|c|c|c|c|c|c|c|}
\hline & A & $\mathrm{C}$ & D & $E$ & $F$ & $\mathbf{G}$ \\
\hline 2 & System & System 1 & System 2 & System 3 & System 4 & System 5 \\
\hline 3 & & Grout Debris & Desorption & Wash & Acid Digestion & CWO \\
\hline 48 & Aqueous Waste Treatment Subsystem & & & & & \\
\hline 49 & Waste Input & & & & & \\
\hline 50 & Aqueous Waste & 60.8 & 60.8 & 60.8 & 60.8 & 60.8 \\
\hline 51 & Process inputs & & & & & \\
\hline 52 & Air (for ozone generation) & 3.5 & 3.8 & 8.6 & 3.8 & 3.9 \\
\hline 53 & $\mathrm{H} 2 \mathrm{O} 2$ & 0.6 & 0.6 & 1.4 & 0.6 & 0.6 \\
\hline 54 & $\mathrm{H} 2 \mathrm{O}$ & 24.1 & 45.8 & 0.5 & 7.6 & 9.0 \\
\hline 55 & $\mathrm{NaOH}$ & 24.1 & 45.8 & 0.5 & 7.6 & 9.0 \\
\hline 56 & input from other subsystems & & & & & \\
\hline 57 & APC - moisture & 0.0 & 0.0 & 0.0 & 0.0 & 0.0 \\
\hline 58 & APC - concentrate & 0.3 & 2.0 & 5.0 & 0.1 & 5.2 \\
\hline 59 & process residue/inorganic sludge & 349.6 & 349.6 & 0.0 & 349.6 & 349.6 \\
\hline 60 & debris treatment & & 32.4 & 129.3 & 432.6 & 406.9 \\
\hline 61 & bulk soil treatment & 40.7 & 40.7 & 355.4 & 355.4 & 355.4 \\
\hline 62 & polymer stabilization & 267.5 & 265.7 & 456.7 & 238.3 & 265.5 \\
\hline 63. & metal decontamination & 126.2 & 126.2 & 126.2 & 126.2 & 126.2 \\
\hline 64 & organic destruction - liquids & 238.9 & 380.3 & 320.3 & 420.3 & 386.1 \\
\hline 65 & organic destruction - salts & 108.6 & & 178.7 & & \\
\hline 66 & Total Subsystem Input & 1244.8 & 1353.8 & 1643.3 & 2002.9 & 1978.1 \\
\hline \multicolumn{7}{|l|}{67} \\
\hline \multicolumn{7}{|c|}{ d Inorganic Sludge Treatment Subsystem } \\
\hline 69 & Waste input & & & & & \\
\hline 70 & Residue, Inorg. Sludge $\quad(3000)$ & 1007.2 & 1007.2 & 1007.2 & 1007.2 & 1007.2 \\
\hline 71 & Process Inputs & & & & & \\
\hline 72 & oxygen & & & & & \\
\hline 73 & N2 sweep gas & 80 & 80 & & 80 & 80 \\
\hline 74 & $\mathrm{H} 2 \mathrm{O}$ & 306 & 306 & 151.05 & 306 & 306 \\
\hline 75 & $\mathrm{NH3}$ & & & & & \\
\hline 76 & $\mathrm{NaOH}$ & & & & & \\
\hline 77 & Input from other subsystems & & & & & \\
\hline 78 & Pb recovery & 13.0 & 13.0 & 13.0 & 13.0 & 13.0 \\
\hline 79 & organic destruction & & & & & \\
\hline 80 & Total Subsystem Input & 1406.1 & 1406.1 & 1171.3 & 1406.1 & 1406.1 \\
\hline \multicolumn{7}{|c|}{81} \\
\hline 82 & Debris Treatment Subsystem & & & & & \\
\hline 83 & Waste Input & & & & & \\
\hline 84 & Debris - Soft & & 434.76 & 434.76 & 434.76 & 434.76 \\
\hline 85 & Debris - Complex & & 177.411 & 177.411 & & \\
\hline 86 & Debris - Open & & 80.4 & 80.4 & 80.4 & 80.4 \\
\hline \multicolumn{7}{|c|}{87 Process Inputs } \\
\hline 88 & Acid-in $(\mathrm{FeCl} 3, \mathrm{H} 2 \mathrm{O}, \mathrm{HCl})$ & & & & & 15.2 \\
\hline 89 & $\mathrm{HNO} 3$ & & & & 42.8 & \\
\hline 90 & oxygen & & & & 791.7 & 766.7 \\
\hline 91 & N2 sweep gas & & 54.9 & & & \\
\hline 92 & $\mathrm{H} 2 \mathrm{O}$ & & 207.9 & 103.8 & 49.7 & 42.9 \\
\hline 93 & $\mathrm{NH3}$ & & & & 3.9 & \\
\hline 94 & $\mathrm{NaOH}$ & & & & 37.7 & 30.9 \\
\hline 95 & Input from other subsystems & & & & & \\
\hline 96 & Pb recovery & & & & & \\
\hline 97 & organic destruction & & & & & \\
\hline 98 & Total Subsystem Input & 0.0 & 955.4 & 796.4 & 1441.0 & 1370.9 \\
\hline
\end{tabular}


Table 6.1

INTS Mass Balance Summary

\begin{tabular}{|c|c|c|c|c|c|c|}
\hline & A & C & D & $\mathbf{E}$ & $\bar{F}$ & $\mathbf{G}$ \\
\hline 2 & System & System 1 & System 2 & System 3: & System 4 & System 5 \\
\hline 3 & & Grout Debris & Desorption & Wash & Acid Digestion & CWO \\
\hline \multicolumn{7}{|c|}{ 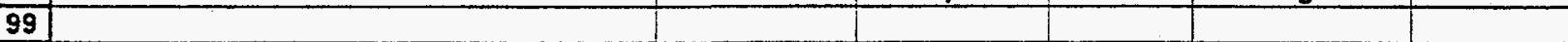 } \\
\hline \multicolumn{7}{|c|}{100 Grout Stabilization Subsystem } \\
\hline \multicolumn{7}{|c|}{101 Waste Input } \\
\hline 102 & Debris - Soft & 434.8 & & & & \\
\hline 103 & Debris-Complex & 177.4 & & & 177.4 & 177.4 \\
\hline 104 & Debris - Open & 80.4 & & & & \\
\hline 105 & Process Inputs & & & & & \\
\hline 106 & dry grout material (cement \& sand) & 1359.6 & 1290.0 & 1274.4 & 365.4 & 904.3 \\
\hline 107 & water required for grout & 471.5 & 246.4 & 498.9 & 152.4 & 383.4 \\
\hline \multicolumn{7}{|c|}{ 108 Input from other subsystems } \\
\hline 109 & bulk soil treatment & 384.8 & 384.8 & 285.5 & & 285.5 \\
\hline 110 & Pb recovery & 5.0 & 5.0 & 5.0 & 5.0 & 5.0 \\
\hline 111 & process residue/inorganic sludge & & & & & \\
\hline 112. & debris treatment & & 838.2 & 667.1 & 82.8 & 182.2 \\
\hline 113 & Total Subsystem Input & 2913.3 & 2764.3 & 2731.0 & 783.1 & 1937.8 \\
\hline \multicolumn{7}{|c|}{114} \\
\hline \multicolumn{7}{|c|}{115 Metal Decontamination Subsystem } \\
\hline \multicolumn{7}{|c|}{ 116 Waste input } \\
\hline 117 & Bulk Metal & 462.11 & 462.1 & 462.1 & 462.1 & 462.1 \\
\hline \multicolumn{7}{|c|}{ 118 Process Inputs } \\
\hline 119 & $\mathrm{H} 2 \mathrm{O}$ & 94.3 & 94.3 & 94.3 & 94.3 & 94.3 \\
\hline \multicolumn{7}{|c|}{\begin{tabular}{l|l}
120 & input from other subsystems \\
\end{tabular}} \\
\hline 121 & Total Subsystem Input & 556.4 & 556.4 & 556.4 & 556.4 & 556.4 \\
\hline \multicolumn{7}{|c|}{ 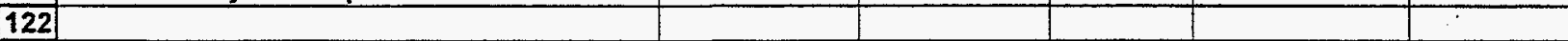 } \\
\hline \multicolumn{7}{|c|}{123 Organic Destruction Subsystem } \\
\hline \multicolumn{7}{|c|}{124 Waste Input } \\
\hline 125 & Organic Liquids, Sluodges $(2000 / 3000)$ & 185.4 & 185.4 & 185.4 & 185.4 & 185.4 \\
\hline \multicolumn{7}{|c|}{126 Process inputs } \\
\hline 127 & $\mathrm{O} 2$ & 566.7 & 701.0 & 775.6 & 575.7 & 604.6 \\
\hline 128 & HNO3 & 21.4 & & 28.8 & 30.9 & \\
\hline 129 & $\mathrm{H} 2 \mathrm{O}$ & 33.7 & 37.8 & 55.5 & 58.6 & 50.7 \\
\hline 130 & $\mathrm{NaOH}$-gas scrubbing & 0.0 & 37.8 & 0.0 & 58.6 & 50.7 \\
\hline 131 & $\mathrm{Ag}$ recovery & 33.7 & & 55.5 & & \\
\hline 132 & $\mathrm{H} 2 \mathrm{O} 2$ & 28.7 & & 47.2 & & \\
\hline 133 & $\mathrm{AgNO3}$ & 2.9 & & 4.7 & & \\
\hline 134 & Acid-in ( $\mathrm{FeCl} 3, \mathrm{H} 2 \mathrm{O}, \mathrm{HCl})$ & & 9.7 & & & 9.3 \\
\hline 135 & $\mathrm{HCl}-\mathrm{Ag}$ precipitation & 0.0 & & 0.0 & & \\
\hline 136 & $\mathrm{NH3}$ & 4.0 & & 5.5 & 2.8 & \\
\hline \multicolumn{7}{|c|}{137 Input from other subsystems } \\
\hline 138 & aqueous waste treatment - decanted orgs & 32.9 & 31.5 & 79.1 & 31.9 & 31.9 \\
\hline 139 & process residue/inorganic sludge & 17.0 & 22.6 & 45.4 & 22.6 & 22.6 \\
\hline 140 & debris treatment & & 27.1 & 0.0 & & \\
\hline 141 & bulk soil treatment & 1.6 & 1.6 & 16.1 & 16.1 & 16.1 \\
\hline 142 & Total Subsystem Input & 928.1 & 1054.5 & 1298.7 & 982.7 & 971.3 \\
\hline \multicolumn{7}{|l|}{143} \\
\hline \multicolumn{7}{|c|}{144 Lead Recovery Subsystem } \\
\hline \multicolumn{7}{|c|}{\begin{tabular}{|l|l|}
145 & Waste Input \\
\end{tabular}} \\
\hline 146 & Lead Waste & 26.0 & 26.0 & 26.0 & 26.0 & 26.0 \\
\hline \multicolumn{7}{|c|}{\begin{tabular}{l|l|}
147 & Process Inputs \\
\end{tabular}} \\
\hline 148 & $\mathrm{H} 2 \mathrm{O}$ & 13 & 13 & 13 & 13 & 13 \\
\hline 149 & Input from other subsystems & & & & & \\
\hline 150 & Total Subsystem input & 39.0 & 39.0 & 39.0 & 39.0 & 39.0 \\
\hline 151 & & & & & & \\
\hline
\end{tabular}


INTS Mass Balance Summary

\begin{tabular}{|c|c|c|c|c|c|c|}
\hline & A & $\mathbf{C}$ & D & \begin{tabular}{|l|}
$E$ \\
\end{tabular} & $\mathbf{F}$ & $\mathbf{G}$ \\
\hline 2 & System & System 1 & System 2 & System 3 & System 4 & System 5 \\
\hline 3 & & Grout Debris & Desorption & Wash & Acid Digestion & CWO \\
\hline \multicolumn{7}{|c|}{152 Polymer Stabilization Subsystem } \\
\hline 153 & Waste input & & & & & \\
\hline 154. & Salt Waste & 6.6 & 6.6 & 6.6 & 6.6 & 6.6 \\
\hline \multirow{2}{*}{$\frac{155}{156}$} & Process Inputs & & & & & \\
\hline & polyethylene or phosphate additive & 801.1 & 843.81 & 788.9 & 6.6 & 861.0 \\
\hline 157 & Input from other subsystems & & & & & \\
\hline 158 & process residue/inorganic sludge & 959.5 & 953.9 & 1125.9 & & 953.9 \\
\hline 159 & aqueous waste treatment - sludge & 102.2 & 125.9 & 108.1 & & 137.7 \\
\hline 160 & aqueous waste treatment - concentrate & 0.3 & 2.0 & 5.0 & & 5.2 \\
\hline 161 & organic destruction & & 21.2 & & & 23.0 \\
\hline 162 & bulk soil treatment & & & & & \\
\hline 163 & debris treatment & & & & & \\
\hline 164 & $\mathrm{APC}$ & 0.0 & 0.0 & 0.0 & & 0.0 \\
\hline 165 & Total Subsystem Input & 1869.7 & 1953.3 & 2034.5 & 13.2 & 1987.5 \\
\hline \multirow{2}{*}{$\frac{166}{167}$} & & & & & & \\
\hline & Phosphate Bonded Ceramic Stabilization & ubsystem & & & & \\
\hline 168 & Waste Input & & & & & \\
\hline \multirow{2}{*}{169} & Process Inputs & & & & & \\
\hline & phosphate additive & & & & 1354.0 & \\
\hline \multirow{2}{*}{$\frac{171}{172}$} & Input from other subsystems & & & & & \\
\hline & process residue/inorganic sludge & & & & 953.9 & \\
\hline \multirow{2}{*}{$\frac{173}{174}$} & aqueous waste treatment - sludge & & & & 172.5 & \\
\hline & aqueous waste treatment - concentrate & & & & 0.1 & \\
\hline 175 & organic destruction & & & & 19.4 & \\
\hline \multirow{2}{*}{$\frac{176}{177}$} & bulk soil treatment & & & & 285.5 & \\
\hline & debris treatment & & & & 160.9 & \\
\hline \multirow{2}{*}{$\frac{178}{175}$} & $\mathrm{APC}$ & & & & 0.0 & \\
\hline & Total Subsystem Input & & & & 2946.3 & \\
\hline \multicolumn{7}{|c|}{ (1) } \\
\hline \multicolumn{7}{|c|}{ 181 Bulk Soil Treatment Subsystem } \\
\hline \multicolumn{7}{|c|}{ 182 Waste Input } \\
\hline 183 & Bulk Soils & 328.5 & 328.5 & 328.5 & 328.5 & 328.5 \\
\hline \multirow{2}{*}{$\frac{184}{185}$} & Process Inputs & & & & & \\
\hline & $\mathrm{H} 2 \mathrm{O}$ & 98.55 & 98.55 & 328.5 & 328.5 & 328.5 \\
\hline 186 & N2 sweep gas & 26 & 26 & & & \\
\hline 187 & Input from other subsystems & & & & & \\
\hline \multirow{2}{*}{$\begin{array}{l}\frac{188}{189} \\
\end{array}$} & Total Subsystem Input & 453.1 & 453.1 & 657.0 & 657.0 & 657.0 \\
\hline & & & & & & \\
\hline 190 & Special Waste Treatment Subsystem & & & & & \\
\hline \multirow{2}{*}{\begin{tabular}{|l|}
191 \\
192 \\
\end{tabular}} & Waste Input & & & & & \\
\hline & Special & 154.9 & 154.9 & 154.9 & 154.9 & 154.9 \\
\hline \multirow{2}{*}{$\frac{193}{194}$} & Process Inputs & & & & & \\
\hline & Input from other subsystems & & & & & \\
\hline 195 & Total Subsystem Input & 154.9 & 154.9 & 154.9 & 154.9 & 154.9 \\
\hline 196 & & & & & & \\
\hline
\end{tabular}


Table 6.1

INTS Mass Balance Summary

\begin{tabular}{|c|c|c|c|c|c|c|}
\hline & A & $\mathrm{C}$ & $D$ & $E$ & $F$ & $\mathbf{G}$ \\
\hline 2 & System & System 1 & System 2 & System 3 & System 4 & System $\mathbf{5}$ \\
\hline 3 & & Grout Debris & Desorption & Wash & Acid Digestion & CWO \\
\hline 197 & 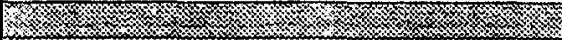 & & \multicolumn{2}{|c|}{ PROCESSOUTPUTS } & (1) & אर \\
\hline 199 & Hg Amalgamation Subsystem & & & & & \\
\hline 200 & Process Outputs. & 4.5 & 8.8 & 4.3 & 4.5 & 4.5 \\
\hline 201 & Output to other subsystems & & & & & \\
\hline 202 & Total Subsystem Output & 4.5 & 8.8 & 4.3 & 4.5 & 4.5 \\
\hline 203 & & & & & & \\
\hline 204 & Air Pollution Control Subsystem & & & & & \\
\hline 205 & Process Outputs & & & & & \\
\hline 206 & Offgas & & & & & \\
\hline 207 & 02 & 29.1 & 35.6 & 55.1 & 10.4 & 30.9 \\
\hline 208 & N2 & 117.9 & 165.1 & 18.9 & 132.1 & 84.2 \\
\hline 209 & NO & 0.8 & 0.7 & 0.8 & 0.2 & 0.7 \\
\hline 210 & $\mathrm{H} 2 \mathrm{O}$ & 9.8 & 1.8 & 15.2 & 10.5 & 3.8 \\
\hline 211 & $\mathrm{CO} 2$ & 537.9 & 619.8 & 737.0 & 1288.0 & 1313.6 \\
\hline 212 & $\mathrm{HNO} 2$ & 0.0 & & 0.0 & & \\
\hline 213 & total offgas & 695.5 & 823.0 & 827.1 & 1441.4 & 1433.3 \\
\hline 214 & Output to other subsystems & & & & & \\
\hline 215 & aqueous waste treatment - salts & 0.3 & 2.0 & 5.0 & 0.1 & 5.2 \\
\hline 216 & aqueous waste treatment - moisture & 0.0 & 0.0 & 0.0 & 0.0 & 0.0 \\
\hline 217 & polymer stabilization - metals & 0.0 & 0.0 & 0.0 & 0.0 & 0.0 \\
\hline 218 & Total Subsystem Output & 695.8 & 825.0 & 832.1 & 1441.5 & 1438.5 \\
\hline 219 & & & & & & \\
\hline 220 & Aqueous Waste Treatment Subsystem & & & & & \\
\hline 221 & Process Outputs & & & & & \\
\hline 222 & aqueous recycle & 1100.8 & 1186.1 & 1423.9 & 1789.9 & 1794.5 \\
\hline 223 & Output to other subsystems & & & & & \\
\hline 224 & organic destruction - decanted orgs & 32.9 & 31.5 & 79.1 & 31.9 & 31.9 \\
\hline 225 & polymer stabilization & 102.2 & 125.9 & 108.1 & 172.5 & 137.7 \\
\hline 226 & polymer stabilization - concentrate & 0.3 & 2.0 & 5.0 & 0.0 & 5.2 \\
\hline 227 & APC & 8.8 & 9.1 & 27.1 & 9.0 & 9.5 \\
\hline 228 & Total Subsystem Output & 1245.1 & 1354.5 & 1643.2 & 2003.4 & 1978.9 \\
\hline 229] & & & & & & \\
\hline 230 & Process Residue and Inorganic Sludge & atment Subsyst & tem & & & \\
\hline 231 & Process Outputs & & & & & \\
\hline 232 & Output to other subsystems & & & & & \\
\hline 233 & polymer stabilization & 959.5 & 953.9 & 1125.9 & 953.9 & 953.9 \\
\hline 234 & organic destruction & 17.0 & 22.6 & 45.4 & 22.6 & 22.6 \\
\hline 235 & aqueous waste treatment & 349.6 & 349.6 & 0.0 & 349.6 & 349.6 \\
\hline 236 & APC & 79.9 & 79.9 & 0.0 & 79.9 & 79.9 \\
\hline 237 & grout stabilization & 0.0 & 0.0 & 0.0 & 0.0 & 0.0 \\
\hline 238 & Hg amalgamation & 0.1 & 0.1 & 0.0 & 0.1 & 0.1 \\
\hline 239 & Total Subsystem Output & 1406.1 & 1406.1 & 1171.3 & 1406.1 & 1406.1 \\
\hline 240 & & & & & & \\
\hline 241 & Debris Treatment Subsystem & & & & & \\
\hline 242 & Process Outputs & & & & & \\
\hline 243 & Output to other subsystems & & & & & \\
\hline 244 & polymer stabilization & 0.0 & & & 160.9 & 0.0 \\
\hline 245 & organic destruction & 0.0 & 27.1 & 0.0 & 0.0 & 0.0 \\
\hline 246 & aqueous waste treatment & 0.0 & 32.4 & 129.3 & 432.6 & 406.9 \\
\hline 247 & APC & 0.0 & 55.2 & 0.0 & 764.8 & 781.8 \\
\hline 248 & grout stabilization & 0.0 & 838.2 & 667.1 & 82.8 & 182.2 \\
\hline 249 & Hg amalgamation & & 2.5 & & & \\
\hline 250 & Total Subsystem Output & 0.0 & 955.4 & 796.4 & 1441.0 & 1370.9 \\
\hline
\end{tabular}




\begin{tabular}{|c|c|c|c|c|c|c|}
\hline & A & $\mathrm{C}$ & D & $E$ & $F$ & $\mathbf{G}$ \\
\hline 2 & System & System 1 & System 2 & System 3 & System 4 & System 5 \\
\hline 3 & & Grout Debris & Desorption & Wash & Acid Digestion & CWO \\
\hline \multicolumn{7}{|c|}{252 Grout Stabilization Subsystem } \\
\hline \multirow{2}{*}{$\frac{253}{254}$} & Process Outputs & & & & & \\
\hline & grouted product & 2913.3 & 2764.3 & 2731.0 & 783.1 & 1937.8 \\
\hline \multirow{2}{*}{$\frac{255}{256}$} & Output to other subsystems & & & & & \\
\hline & Total Subsystem Output & 2913.3 & 2764.3 & 2731.0 & 783.1 & 1937.8 \\
\hline $258 \mid 1$ & Metal Decontamination Subsystem & & & & & \\
\hline 259 & Process Outputs & & & & & \\
\hline 260 & decontaminated metal & 430.3 & 430.3 & 430.3 & 430.3 & 430.3 \\
\hline \multirow{2}{*}{\begin{tabular}{|l|}
261 \\
262
\end{tabular}} & Output to other subsystems & & & & & \\
\hline & aqueous waste treatment & 126.2 & 126.2 & 126.2 & 126.2 & 126.2 \\
\hline 263 & Total Subsystem Output & 556.4 & 556.4 & 556.4 & 556.4 & 556.4 \\
\hline \multicolumn{7}{|c|}{ 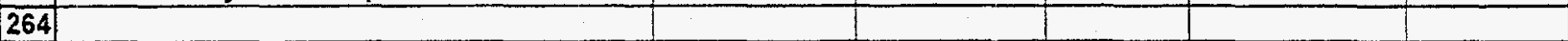 } \\
\hline \multicolumn{7}{|c|}{265 Organic Destruction Subsystem } \\
\hline \multicolumn{7}{|c|}{ 266) Process Outputs } \\
\hline \multirow{2}{*}{\begin{tabular}{|l|}
267 \\
268
\end{tabular}} & Output to other subsystems & & & & & \\
\hline & process residue/inorganic sludge & 0.0 & 0.0 & 0.0 & 0.0 & 0.0 \\
\hline 269 & aqueous waste treatment - water & 238.9 & 380.3 & 320.3 & 420.3 & 386.1 \\
\hline \multirow{2}{*}{$\frac{270}{271}$} & aqueous waste treatment - salts & 108.6 & 0.0 & 178.7 & 0.0 & 0.0 \\
\hline & APC-gases & 580.5 & 652.6 & 799.8 & 542.4 & 561.6 \\
\hline \multirow{2}{*}{$\frac{272}{273}$} & polymer stabilization & 0.0 & 21.2 & 0.0 & 19.4 & 23.0 \\
\hline & Total Subsystem Output & 928.1 & 1054.1 & 1298.8 & 982.1 & 970.8 \\
\hline \multicolumn{7}{|l|}{274} \\
\hline $275 \mid$ & Lead Recovery Subsystem & & & & & \\
\hline \multirow{2}{*}{\begin{tabular}{|l|}
276 \\
277
\end{tabular}} & Process Outputs & & & & & \\
\hline & lead out & 21 & 21 & 21 & 21 & 21 \\
\hline 278 & Output to other subsystems & & & & & \\
\hline 279 & process residue/inorganic sludge & 13.0 & 13.0 & 13.0 & 13.0 & 13.0 \\
\hline 280 & grout - gloves & 5.0 & 5.0 & 5.0 & 5.0 & 5.0 \\
\hline 281 & Total Subsystem Output & 39.0 & 39.0 & 39.0 & 39.0 & 39.0 \\
\hline 282 & & & & & & \\
\hline 283 & Polymer Stabilization Subsystem & & & & & \\
\hline 284 & Process Outputs & & & & & \\
\hline 285 & polymer stabilized waste & 1602.2 & 1687.6 & 1577.8 & 13.2 & 1722.0 \\
\hline 286 & Output to other subsystems & & & & & \\
\hline 287 & aqueous waste treatment & 267.5 & 265.7 & 456.7 & & 265.5 \\
\hline 288 & Total Subsystem Output & 1869.7 & 1953.3 & 2034.5 & 13.2 & 1987.5 \\
\hline 289 & & & & & & \\
\hline 290 & Phosphate Bonded Ceramic Stabilization St & ubsystem & & & & \\
\hline 291 & Process Outputs & & & & & \\
\hline 292 & phosphate bonded ceramic stabilized waste & & & & 2707.9 & \\
\hline 293 & Output to other subsystems & & & & & \\
\hline 294 & aqueous waste treatment & & & & 238.3 & \\
\hline 295 & Total Subsystem Output & & & & 2946.3 & \\
\hline 296 & & & & & & \\
\hline 297 & Bulk Soil Treatment Subsystem & & & & & \\
\hline 298 & Process Outputs & & & & & \\
\hline 299 & Output to other subsystems & & & & & \\
\hline 300 & $\mathrm{APC}$ & 26.1 & 26.1 & 0.0 & 0.0 & 0.0 \\
\hline 301 & aqueous waste treatment & 40.7 & 40.7 & 355.4 & 355.4 & 355.4 \\
\hline 302 & grout & 384.8 & 384.8 & 285.5 & 0.0 & 285.5 \\
\hline 303 & organic destruction & 1.6 & 1.6 & 16.1 & 16.1 & 16.1 \\
\hline 304 & polymer stabilization & 0.0 & 0.0 & 0.0 & 285.5 & 0.0 \\
\hline 305 & Total Subsystem Output & 453.1 & 4.53 .1 & 657.0 & 657.0 & 657.0 \\
\hline
\end{tabular}


Table 6.1

INTS Mass Balance Summary

\begin{tabular}{|c|c|c|c|c|c|c|}
\hline & A & C & D & E & $F$ & $\mathbf{G}$ \\
\hline 2 & System & System 1 & System 2 & System 3 & System 4 & System 5 \\
\hline 3 & & Grout Debris & Desorption & Wash & Acid Digestion & CWO \\
\hline \multicolumn{7}{|l|}{306} \\
\hline \multicolumn{7}{|c|}{ 307|Special Waste Treatment Subsystem } \\
\hline 308 & Process Outputs. & 154.9 & 154.9 & 154.9 & 154.9 & 154.9 \\
\hline 309 & Output to other subsystems & & & & & \\
\hline 310 & Total Subsystem Output & 154.9 & 154.9 & 154.9 & 154.9 & 154.9 \\
\hline \multicolumn{7}{|l|}{311} \\
\hline 321 & Reagents Input to Process (density, Ib/ft3) & & & & & \\
\hline 322 & Cu for $\mathrm{Hg}$ amalgamation & 1.9 & 3.6 & 1.8 & 1.8 & 1.9 \\
\hline 323 & $\mathrm{NaOH}$ & 58.0 & 84.7 & 58.5 & 104.0 & 93.4 \\
\hline 324 & acid solution $(\mathrm{FeCl} 3, \mathrm{H} 2 \mathrm{O}, \mathrm{HCl})(-62)$ & 0.0 & 9.7 & 0.0 & 0.0 & 24.5 \\
\hline 325 & NH3 $\quad$ (45) & 4.0 & 0.0 & 5.5 & 6.8 & 0.0 \\
\hline 326 & HNO3 & 21.4 & 0.0 & 28.8 & 73.7 & 0.0 \\
\hline 327 & $\mathrm{AgNO} 3$ & 2.9 & 0.0 & 4.7 & 0.0 & 0.0 \\
\hline 328 & $\mathrm{H} 2 \mathrm{O} 2$ & 29.2 & 0.6 & 48.6 & 0.6 & 0.6 \\
\hline 329 & grout material & 1359.6 & 1290.0 & 1274.4 & 365.4 & 904.3 \\
\hline 330 & water required for grout $\quad$ (62) & 471.5 & 246.4 & 498.9 & 152.4 & 383.4 \\
\hline 331 & polyethylene/phosphate ceramic $(\sim 80)$ & 801.1 & 843.8 & 788.9 & 1360.6 & 861.0 \\
\hline 332 & & & & & & \\
\hline
\end{tabular}


Table 6.1

INTS Mass Balance Summary

\begin{tabular}{|c|c|c|c|c|c|c|}
\hline & A & $\mathrm{C}$ & $\mathrm{D}$ & $\mathbf{E}$ & $F$ & $\mathbf{G}$ \\
\hline 2 & System & System 1 & System 2 & System 3: & System 4 & System 5 \\
\hline 3 & & Grout Debris & Desorption & Wash & Acid Digestion & CWO \\
\hline 333 & 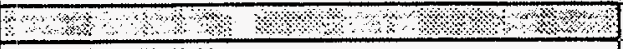 & \multicolumn{4}{|c|}{ MASS AND YOLUME REOUCTION CALCULATION } & 16/ \\
\hline 334 & Densities (lb/ft3) & & & & & \\
\hline 335 & Input Waste & 64 & 64 & 64 & 64 & 64 \\
\hline 336 & Soil & 67 & 67 & 67 & 67 & 67 \\
\hline 337 & Metal (Input) & 480 & 480 & 480 & 480 & 480 \\
\hline 338 & Metal (Output) & 480 & 480 & 480 & 480 & 480 \\
\hline 339 & Aqueous Liquid & 62 & 62 & 62 & 62 & 62 \\
\hline 340 & Slag & 187 & 187 & 187 & 187 & 187 \\
\hline 341 & Polymerized Salt & 80 & 80 & 80 & 80 & 80 \\
\hline 342 & Polymerized Ash/Metal/Salt & 94 & 94 & 94 & 94 & 94 \\
\hline 343 & Polymerized Lime & 96.5 & 96.5 & 96.5 & 96.5 & 96.5 \\
\hline 344 & Grouted Debris & 126.6 & 126.6 & 126.6 & 126.6 & 126.6 \\
\hline 345 & Stabilized $\mathrm{Hg}$ Amalgam & 733.0 & 733.0 & 733.0 & 733.0 & 733.0 \\
\hline \multirow{2}{*}{\multicolumn{7}{|c|}{347 Mass Flow Rate Calculation }} \\
\hline & & & & & & \\
\hline 348 & Mass Solids In (lb/hr, no aqueous) & 2865.8 & 2865.8 & 2865.8 & 2865.8 & 2865.8 \\
\hline 349 & Mass Grouted Waste Out (lb/hr) & 2913.3 & 2764.3 & 2731.0 & 783.1 & 1937.8 \\
\hline 350 & Mass Special Waste (not stabilized) & 154.9 & 154.9 & 154.9 & 154.9 & 154.9 \\
\hline 351 & Mass Metal/Pb Out (Ib/hr)clean & 451.3 & 451.3 & 451.3 & 451.3 & 451.3 \\
\hline 352 & Mass Polymerized Waste Out $(\mathrm{l} / \mathrm{h} /)$ & 1602.2 & 1687.6 & 1577.8 & 13.2 & 1722.0 \\
\hline 353 & Mass Phosphate Bonded Waste Out ( $(\mathrm{b} / \mathrm{hr})$ & & & & 2707.9 & \\
\hline 354 & Mass Stabilized Hg Amalgam Out & 4.5 & 8.8 & 4.3 & 4.5 & 4.5 \\
\hline 355 & $\begin{array}{l}\text { Total Mass Processed Wastes Out (lb/hr, no } \\
\text { metals) }\end{array}$ & 4675 & 4616 & 4468 & 3664 & 3819 \\
\hline 356 & Waste Mass Reduction Ratio (In/Out) & 0.61 & 0.62 & 0.64 & 0.78 & 0.75 \\
\hline \multicolumn{7}{|c|}{ 358 Volumetric Flow Rate $\mathrm{C}$} \\
\hline 359 & Volume Solids In (ft3/hr) & 45 & 45 & 45 & 45 & 45 \\
\hline 360 & Volume Grouted Waste Out (ft3/hr) & 23.0 & 21.8 & 21.6 & 6.2 & 15.3 \\
\hline 361 & $\begin{array}{l}\text { Volume Special Wastes (not stabilized) } \\
\text { (ft3/hr) }\end{array}$ & 2.4 & 2.4 & 2.4) & 2.4 & 2.4 \\
\hline 362 & Volume Metal/Pb Out (ft $3 / \mathrm{hr})$ & 0.9 & 0.9 & 0.9 & 0.9 & 0.9 \\
\hline 363 & Volume Polymerized Waste Out (ft3/hr) & 17.0 & 18.0 & 16.8 & 0.1 & 18.3 \\
\hline 364 & Volume Phosphate Waste Out (ft3/hr) & & & & 28.8 & \\
\hline 365 & Vol Stabilized Hg Amalgam Out & 0.0 & 0.0 & 0.0 & 0.0 & 0.0 \\
\hline 366 & $\begin{array}{l}\text { Total Volume Solids Out to Disposal } \\
\text { (ft3/hr, no metals) }\end{array}$ & 42.5 & 42.2 & 40.8 & 37.6 & 36.1 \\
\hline 367 & Volume Metal to Recycle (ft $3 / \mathrm{hr}$ ) & 0.9 & 0.9 & 0.9 & 0.9 & 0.9 \\
\hline 368 & Waste Volume Reduction Ratio (In/Out) & $5.7 \times 1.05$ & $10 \times 1.06$ & 3.1 .10 & $10 \times 1.19$ & $18 \times 1.24$ \\
\hline 370 & Total Water Requirements & & & & & \\
\hline 371 & Aqueous Waste Treatment & 24 & 46 & 1) & 8 & 9 \\
\hline 372 & Inorganic Sludge Washing / $\mathrm{Hg}$ Leaching & 306 & 306 & 151 & 306 & 306 \\
\hline 373 & Lead Recovery & 13 & 13 & 13 & 13 & 13 \\
\hline 374 & Debris Treatment / Hg Leaching & of & 208 & 104 & 50 & 43 \\
\hline 375 & Metal Decontamination & 94 & 94 & 94 & 94 & 94 \\
\hline 376 & Organic Destruction & 34 & 38 & 55 & 59 & 51 \\
\hline 377 & Soil Washing / Hg Leaching & 99 & 99 & 329 & 329 & 329 \\
\hline 378 & Air Pollution Control - scrubber water & 0 & 1 & 3 & of & 3 \\
\hline 379 & Water for grout ( 30 weight $\%$ ) & 471 & 246 & 499 & 152 & 383 \\
\hline 380 & Total & 1041 & 1051 & 1248 & 1010 & 1231 \\
\hline 381 & Total Water Available for Recycle & 1101 & 1186 & 1424 & 1790 & 1795 \\
\hline 382 & Net Water Generation Rate (recycie - required) & 59 & 135 & 176 & 780 & 564 \\
\hline
\end{tabular}




\begin{tabular}{|c|c|c|c|c|c|c|}
\hline \multicolumn{6}{|c|}{ Energy Requirements of Waste Treatment for Five INTS Systems (Btu/h) } & \\
\hline & & & \multicolumn{3}{|c|}{ (basis: $2927 \mathrm{lb} / \mathrm{h}$ waste feed) } & \\
\hline & & & & & & \\
\hline & & System 1 & System 2 & System 3 & System 4 & System 5 \\
\hline & Energy Type & Baseline & Desorption & Wash & Acid Digestion & Combination \\
\hline NOx removal & cooling water & $-78.8 \mathrm{E}+3$ & & $-107.7 E+3$ & & \\
\hline Gas Phase Corona Reactor (GPCR) & electricity & $20.6 \mathrm{E}+3$ & $24.4 E+3$ & $22.9 E+3$ & $40.3 \mathrm{E}+3$ & $39.1 E+3$ \\
\hline Neutral3 - acid gas neutralization & cooling water & $-12.1 E+3$ & $-52.2 E+3$ & $-11.2 E+3$ & $-105.1 E+3$ & $-98.6 E+3$ \\
\hline HEPA preheaters & gas & $31.6 \mathrm{E}+3$ & $37.1 E+3$ & $37.1 E+3$ & $64.4 E+3$ & $63.4 E+3$ \\
\hline Ozone Generation for photo-UV & electricity & $1.1 \mathrm{E}+3$ & $1.2 E+3$ & $2.6 E+3$ & $1.2 E+3$ & $1.2 E+3$ \\
\hline $\mathrm{OH}$-generator (from $\mathrm{O} 3$ and $\mathrm{H} 2 \mathrm{O} 2$ ) & cooling water & $-82.2 E+3$ & $11.7 \mathrm{E}+3$ & $-160.1 E+3$ & $2.7 E+3$ & $1.1 E+3$ \\
\hline Photo-UV reactor & cooling water & $-17.7 E+3$ & $-18.2 E+3$ & $-42.6 E+3$ & $-24.4 E+3$ & $-22.8 E+3$ \\
\hline Ozone decomposition & cooling water & $-46.4 E+3$ & $-91.6 E+3$ & $-2.1 E+3$ & $-17.0 E+3$ & $-222 E+3$ \\
\hline Salt concentrator (evaporator) & electricity & $10.0 E+0$ & $43.0 E+0$ & $111.0 \mathrm{E}+0$ & $2.0 E+0$ & $112.0 E+0$ \\
\hline Vacuum thermal desorption of inorganic sludge & gas & $540.2 E+3$ & $515.6 E+3$ & & $515.6 E+3$ & $515.6 \mathrm{E}+3$ \\
\hline HNO2 recovery & cooling water & $-736.0 E+3$ & & $-1.0 E+6$ & $-1.1 E+6$ & \\
\hline MEO reactor - anode & electricity & $4.6 \mathrm{E}+6$ & & $6.4 \mathrm{E}+6$ & & \\
\hline MEO reactor - cathode & cooling water & & & & & \\
\hline MEO reactor - Ag ppt & cooling water & & & & & \\
\hline Ag recovery from MEO process & cooling water & $140.4 E+3$ & & $761.4 \mathrm{E}+3$ & & \\
\hline Dryer (before polymer stabilization) & gas if +10 if - & $-19.5 E+3$ & $79.3 E+3$ & $91.4 E+3$ & $104.0 E+3$ & $14.6 E+3$ \\
\hline Vacuum thermal desorption of soil & gas & $54.9 E+3$ & $54.9 E+3$ & & & \\
\hline Vacuum thermal desorption of debris & gas & & $518.6 E+3$ & & & \\
\hline Catalytic wet oxidation of organic liquids & cooling water & & $-3.7 E+6$ & & & $-3.3 E+6$ \\
\hline gas equilibration after organic destruction & cooling water & & $-7.3 E+3$ & & & $-11.9 \mathrm{E}+3$ \\
\hline gas equilibration after debris treatment & cooling water & & & & & $506.0 \mathrm{E}+0$ \\
\hline acid gas neutralization after organic destruction ( & cooling water & $-265.0 E+0$ & $-364.0 E+3$ & $-406.0 E+0$ & $-419.8 E+3$ & $-351.8 E+3$ \\
\hline Catalytic wet oxidation of debris & cooling water & & & & & $-4.6 E+6$ \\
\hline Acid digestion of debris & cooling water & & & & $-29.3 E+6$ & \\
\hline HNO2 recovery after debris treatment & cooling water & & & & $-1.5 E+6$ & \\
\hline acid gas neutralization after debris treatment & cooling water & & & & $-470.4 E+3$ & $-388.0 E+3$ \\
\hline Acid digestion of organic liquids & cooling water & & & & $-2.2 E+6$ & \\
\hline Soll dryer after washing & gas & & & $54.6 \mathrm{E}+3$ & $54.6 \mathrm{E}+3$ & $54.6 \mathrm{E}+3$ \\
\hline totals (Btu) & electricity & $4.6 E+6$ & $25.6 \mathrm{E}+3$ & $6.4 E+6$ & $41.5 \mathrm{E}+3$ & $40.5 E+3$ \\
\hline & gas & $626.8 E+3$ & $1.2 \mathrm{E}+6$ & 183.1E+3 & $738.6 E+3$ & $648.2 E+3$ \\
\hline & cooling water & $-833.2 E+3$ & $-4.2 E+6$ & $-568.7 E+3$ & $-35.1 E+6$ & $-8.8 E+6$ \\
\hline & & & & & & \\
\hline Costs $(\$)$ & $e(\$ 0.05 / \mathrm{kWh})$ & $\$ 67.98$ & $\$ .38$ & $\$ 93.52$ & $\$ .61$ & $\$ .59$ \\
\hline & $g(\$ 2.00$ MMBtu $)$ & $\$ 1.25$ & $\$ 2.41$ & $\$ .37$ & $\$ 1.48$ & $\$ 1.3$ \\
\hline & $\mathrm{CW}(\mathrm{SO})$ & $\$$. & $\$$. & $\$$. & $\$$. & $\$$ \\
\hline total & & $\$ 69.23$ & $\$ 2.79$ & $\$ 93.89$ & $\$ 2.08$ & $\$ 1.89$ \\
\hline - energy requirements for MEO process are & $63 / \mathrm{kg}$ waste @ \$0 & & & & & \\
\hline
\end{tabular}




\section{REFERENCES}

Biagi, Charles et al., "Integrated Nonthermal Treatment System Study, Final Draft," INEL/EXT-96-0273, Idaho National Engineering Laboratory, Idaho Falls, ID, January 1997

Chiba, Z, letter to M. Adamson, "Loss of Silver in the Mediated Electrochemical Oxidation Process," Lawrence Livermore National Laboratory, January 11, 1996.

Dhooge, P. M. and L. B. Hakim, Development Studies for a Novel Wet Oxidation Process, Delphi Research, Inc., DOE Contract DE-AC21-92MC29107, January 1994, p. 8.

Lawrence Livermore National Laboratory, Mediated Electrochemical Oxidation Treatment for Rocky Flats Combustible Low-Level Mixed Waste, FY1994 Final Report, CRL-ID-118679, September 1994.

Liebelt, K. H. , B. W. Brown, and W. J. Quapp, Process Modeling for the Integrated Thermal Treatment System (ITTS) Study, Idaho National Engineering Laboratory, INEL-95/0335, September 1995.

Pierce, R. A. and J. R. Smith, "Nitric-Phosphoric Acid Oxidation of Organic Waste Materials," Westinghouse Savannah River Company, presented at ASME Mixed Waste Symposium, August 1995.

Schwinkendorf, William E.et al., Alternatives to Incineration Technical Area Status Report, BDM Federal, Albuquerque, NM, April 1995. 
Appendix A

Model Assumptions and ASPEN PLUS

Process Flow Diagrams 
System 1 - Grout Debris Case

\begin{tabular}{|c|c|c|c|}
\hline \multirow{2}{*}{\multicolumn{4}{|c|}{$\begin{array}{l}\text { ASSUMPTIONS and ENERG } \\
\text { GENERAL COMMENTS: }\end{array}$}} \\
\hline & & & \\
\hline $\begin{array}{l}\text { Unit Operation / } \\
\text { ASPEN PLUS } \\
\text { Block Name }\end{array}$ & $\begin{array}{l}\text { Outlet } \\
\text { Temp. } \\
\left({ }^{\circ} \mathrm{F}\right)\end{array}$ & $\begin{array}{l}\text { Heat Duty } \\
\text { (MMBtu/hr) }\end{array}$ & Description and Assumptions \\
\hline $\begin{array}{l}\text { APC filter / } \\
\text { FIL TER (SEP) }\end{array}$ & 65 & - & $\begin{array}{l}\text { Filter to separate heavy metals and other solids } \\
\text { from the gas stream. Gas streams entering the } \\
\text { APC system usually have all particulates removed } \\
\text { beforehand. }\end{array}$ \\
\hline $\begin{array}{l}\text { Gas phase corona } \\
\text { reactor / } \\
\text { GPCR (RSTOIC) }\end{array}$ & 180 & 20649 & 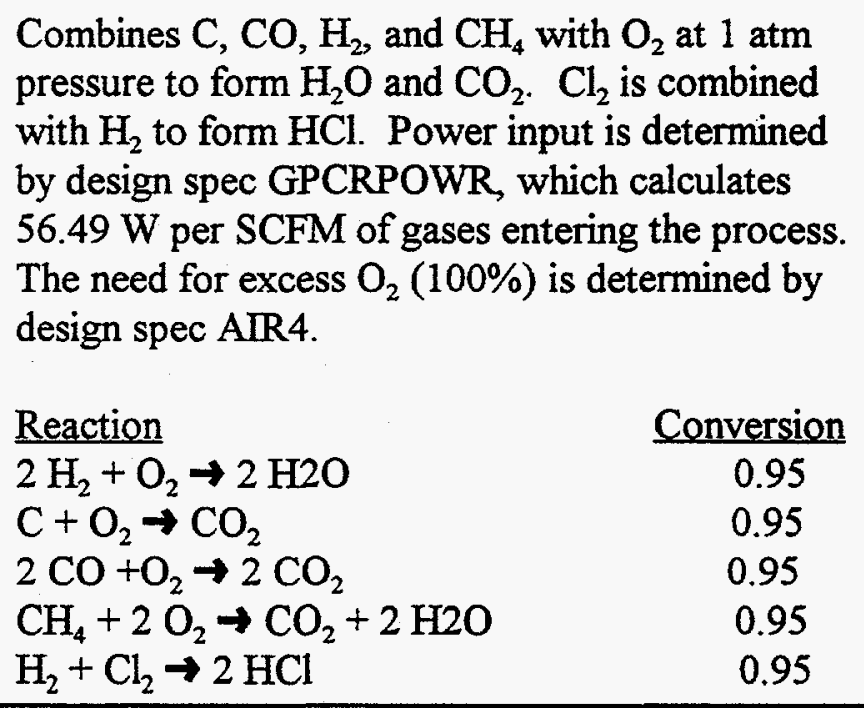 \\
\hline $\begin{array}{l}\text { Acid gas scrubber } \\
\text { / NEUTRAL3 } \\
\text { (RSTOIC) }\end{array}$ & 100 & -12149 & $\begin{array}{l}\text { Neutralizes } \mathrm{SO}_{2} \text { with } \mathrm{NaOH} \text { at } 1 \text { atm pressure and } \\
100^{\circ} \mathrm{T} \text { to form } \mathrm{Na}_{2} \mathrm{SO}_{4} \text { at a conversion level of } \\
99 \% \text {. Excess } \mathrm{NaOH}(1 \%) \text { is determined by } \\
\text { design spec } \mathrm{NAOH}-3 \text {. } \\
\text { Reaction } \\
2 \mathrm{SO}_{2}+4 \mathrm{NaOH}+\mathrm{O}_{2} \rightarrow 2 \mathrm{Na}_{2} \mathrm{SO}_{4}+2 \mathrm{H}_{2} \mathrm{O} \quad 0.99\end{array}$ \\
\hline $\begin{array}{l}\text { Scrubber liquor } \\
\text { sump / } \\
\text { BLOWDOWN } \\
\text { (SEP) }\end{array}$ & 100 & - & $\begin{array}{l}\text { Separates salts formed in the scrubber. } \mathrm{Na}_{2} \mathrm{SO}_{4} \\
\text { and } \mathrm{NaOH} \text { are sent with some water to the } \\
\text { aqueous waste treatment subsystem. Water flow } \\
\text { rate calculated for } 20 \% \text { salt solution by design } \\
\text { spec SALTSOLN. }\end{array}$ \\
\hline
\end{tabular}




\begin{tabular}{|c|c|c|c|}
\hline $\begin{array}{l}\text { Mist eliminator / } \\
\text { FLASH } \\
\text { (FLASH2) }\end{array}$ & 100 & 0 & $\begin{array}{l}\text { Two-phase flash to separate out additional } \\
\text { moisture. Flash determines vapor-liquid } \\
\text { equilibrium at } 100^{\circ} \mathrm{F} \text { and } 1 \text { atm. Moisture sent to } \\
\text { the aqueous waste treatment subsystem. }\end{array}$ \\
\hline $\begin{array}{l}\text { Filter / } \\
\text { HETRHEPA } \\
\text { (HEATER) }\end{array}$ & 300 & 31630 & $\begin{array}{l}\text { Heater to raise temperature of the gas stream to } \\
300^{\circ} \mathrm{F} \text { to avoid condensate in the HEPA filter. }\end{array}$ \\
\hline $\begin{array}{l}\text { Ozone generator / } \\
\text { OZ-GEN } \\
\text { (RSTOIC) }\end{array}$ & 100 & 1053 & $\begin{array}{l}\mathrm{O}_{3} \text { generated from } \mathrm{O}_{2} \text { at } 100^{\circ} \mathrm{F} \text { and } 1 \mathrm{~atm} . \\
\frac{\text { Reaction }}{3 \mathrm{O}_{2} \rightarrow 2 \mathrm{O}_{3}} \quad \frac{\text { Conversion }}{0.99}\end{array}$ \\
\hline $\begin{array}{l}\text { Aqueous liquids } \\
\text { sorting / } \\
\text { PHOTOMIX } \\
\text { (MIXER) }\end{array}$ & 94 & - & $\begin{array}{l}\text { Combines all streams entering the aqueous waste } \\
\text { treatment subsystem. }\end{array}$ \\
\hline $\begin{array}{l}\text { Gross organics } \\
\text { removal / } \\
\text { OLLSEP (SEP) }\end{array}$ & 94 & - & $\begin{array}{l}\text { Oil-water separator to remove } \mathrm{C} \text { and } \mathrm{H}_{2} \text { from the } \\
\text { liquid stream at an assumed efficiency of } 99 \% \text {. }\end{array}$ \\
\hline $\begin{array}{l}\text { OH generator / } \\
\text { OH-GEN } \\
\text { (RSTOIC) }\end{array}$ & 100 & -82246 & 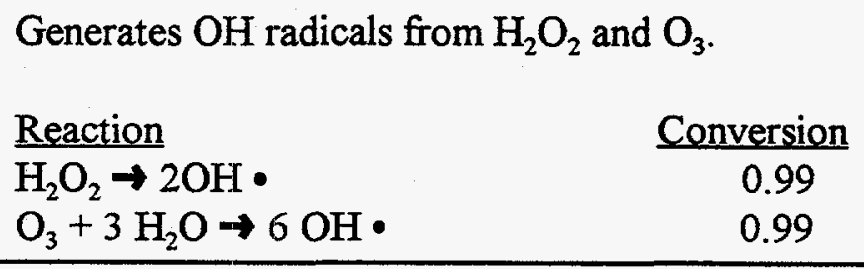 \\
\hline $\begin{array}{l}\text { UV reactor / } \\
\text { PHOTO-UV } \\
\text { (RSTOIC) }\end{array}$ & 100 & -17740 & 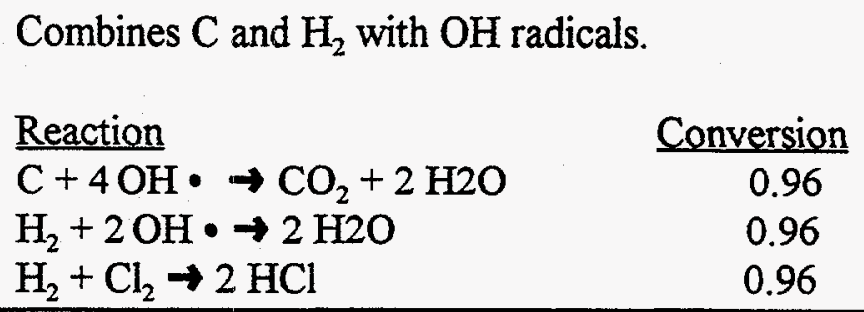 \\
\hline
\end{tabular}




\begin{tabular}{|c|c|c|c|}
\hline $\begin{array}{l}\text { Ozone } \\
\text { decomposition / } \\
\text { OZ-DECOM } \\
\text { (RSTOIC) }\end{array}$ & 100 & -46396 & $\begin{array}{l}\text { Decomposes unreacted ozone and neutralizes } \\
\text { acids at } 100^{\circ} \mathrm{F} \text { and } 1 \mathrm{~atm} \text {. Excess } \mathrm{NaOH}(1 \%) \text { is } \\
\text { determined by design spec XX-NAOH. } \\
\begin{array}{lc}\text { Reaction } & \text { Conversion } \\
2 \mathrm{O}_{3} \rightarrow 3 \mathrm{O}_{2} & 0.999 \\
\mathrm{NaOH}+\mathrm{HCl} \rightarrow \mathrm{NaCl}+\mathrm{H} 2 \mathrm{O} & 0.99 \\
4 \mathrm{NaOH}+2 \mathrm{SO}_{2}+\mathrm{O}_{2} \rightarrow 2 \mathrm{Na}_{2} \mathrm{SO}_{4}+2 \mathrm{H}_{2} \mathrm{O} & 0.99 \\
4 \mathrm{NaOH}+2 \mathrm{Cl}_{2} \rightarrow 4 \mathrm{NaCl}_{2}+\mathrm{H}_{2} \mathrm{O}+\mathrm{O}_{2} & 0.99 \\
2 \mathrm{NaOH}+\mathrm{H}_{2} \mathrm{SO}_{4} \rightarrow \mathrm{Na}_{2} \mathrm{SO}_{4}+2 \mathrm{H}_{2} \mathrm{O} & 0.99 \\
\end{array}\end{array}$ \\
\hline $\begin{array}{l}\text { Gas, water, } \\
\text { sludge separator / } \\
\text { AQ-SPLIT (SEP) }\end{array}$ & 100 & - & $\begin{array}{l}\text { Separates gas, liquid, and sludge from aqueous } \\
\text { waste treatment. Gases }\left(\mathrm{O}_{2}, \mathrm{H}_{2}, \mathrm{~N}_{2}, \mathrm{NO}, \mathrm{CO}_{2} \text {, }\right. \\
\left.\mathrm{O}_{3}\right) \text { are sent to the } \mathrm{APC} \mathrm{subsystem.} \mathrm{Liquids} \\
\left(\mathrm{H}_{2} \mathrm{O}, \mathrm{SO}_{2}, \mathrm{FeCl}_{3}, \mathrm{H}_{2} \mathrm{SO}_{4}, \mathrm{HNO}_{3}, \mathrm{H}^{+}, \mathrm{e}^{-}, \mathrm{H}_{2} \mathrm{O}_{2},\right. \\
\left.\mathrm{OH} \text { radicals, } 1 \% \text { of C, } 1 \% \text { of } \mathrm{SiO}_{2}, 1 \% \text { of } \mathrm{Al}_{2} \mathrm{O}_{3}\right) \\
\text { are available for recycle in the system. Solids }(\mathrm{Fe} \text {, } \\
\mathrm{As}, \mathrm{Ba}, \mathrm{Cd}, \mathrm{Cr}, \mathrm{Pb}, \mathrm{Se}, \mathrm{Ag}, \mathrm{NaCl}, \mathrm{AgCl}, \mathrm{NaNO}_{3} \text {, } \\
\left.\mathrm{Na}_{2} \mathrm{SO}_{4}, 99 \% \text { of C, } 99 \% \text { of } \mathrm{SiO}_{2}, 99 \% \text { of } \mathrm{Al}_{2} \mathrm{O}_{3}\right) \\
\text { are routed to polymer stabilization subsystem. }\end{array}$ \\
\hline $\begin{array}{l}\text { Evaporator / } \\
\text { CONCENT8 } \\
\text { (FLASH2) }\end{array}$ & 150 & 10 & $\begin{array}{l}\text { Models evaporation of scrubber liquor from APC } \\
\text { subsystem. Two-phase flash determines vapor- } \\
\text { liquid equilibrium at } 150^{\circ} \mathrm{F} \text { and } 1 \text { atm. Vapor } \\
\text { sent to PHOTOMIX, liquid/solid phase sent to } \\
\text { polymer stabilization subsystem. }\end{array}$ \\
\hline $\begin{array}{l}\text { Soil dryer / } \\
\text { VACTRAX3 } \\
\text { (RGIBBS) }\end{array}$ & 550 & 54911 & $\begin{array}{l}\text { Models the vacuum desorber for bulk soils. } \\
\text { Operates at } 550^{\circ} \mathrm{F} \text { and } 1 \text { psig. Equilibrium is } \\
\text { assumed for species entered in the RGIBBS } \\
\text { block: } \mathrm{HCl} \text { and } \mathrm{CH}_{4} \text { are assumed to form from } \\
\text { feed components. } \mathrm{O}_{2} \text { is excluded from reaction. } \\
\text { A nitrogen sweep gas is used at a rate of } 1.09 \mathrm{ft}^{3} \\
\text { per lb waste ( }\left(0.0793 \mathrm{lb} \mathrm{N} \mathrm{N}_{2} / \mathrm{b} \text { waste) as }\right. \\
\text { determined by FORTRAN block N2SWEEP. }\end{array}$ \\
\hline
\end{tabular}




\begin{tabular}{|c|c|c|c|}
\hline $\begin{array}{l}\text { Mercury leaching } \\
\text { / VACSEP-3 } \\
\text { (SEP) }\end{array}$ & 91 & - & $\begin{array}{l}\text { Mercury leaching of soils by addition of leach } \\
\text { water ( } 30 \% \text { of mass of incoming waste). Also } \\
\text { includes separation of gases, liquids, organics, } \\
\text { Hg-contaminated liquids, and solids. } \\
\text { Gases }\left(\mathrm{N}_{2}\right) \text { are sent to APC subsystem. Solids } \\
\left(\mathrm{H}_{2} \mathrm{O} \text { in leach water, } 50 \% \text { of } \mathrm{H}_{2}, 50 \% \text { of } \mathrm{C}, \mathrm{SiO}_{2} \text {, }\right. \\
\left.\mathrm{Al}_{2} \mathrm{O}_{3}\right) \text { are routed to grout stabilization } \\
\left.\text { subsystem. Liquids (balance of } \mathrm{H}_{2} \mathrm{O}, \mathrm{HCl}\right) \text { are sent } \\
\text { to aqueous waste treatment. Organics }(50 \% \text { of } \\
\mathrm{H}_{2}, \mathrm{CH}_{4}, 50 \% \text { of } \mathrm{C} \text { ) are routed to the organic } \\
\text { destruction subsystem. }\end{array}$ \\
\hline $\begin{array}{l}\text { Grout } \\
\text { stabilization feed } \\
\text { preparation / } \\
\text { GROUTMIX } \\
\text { (MIXER) }\end{array}$ & 74 & - & $\begin{array}{l}\text { Combines all waste feed to grout stabilization, } \\
\text { including soft, open, and complex debris. }\end{array}$ \\
\hline $\begin{array}{l}\text { Micro- } \\
\text { solidification / } \\
\text { GROUTER } \\
\text { (MIXER) }\end{array}$ & 68 & - & $\begin{array}{l}\text { Combines cement and water with incoming waste } \\
\text { feed. Amount of dry cement is determined by } \\
\text { design spec GROUT. Total grout:waste ratio is } \\
2: 1 \text { and dry grout:water ratio is } 0.7: 0.3 \text {. Amount } \\
\text { of water is determined by design spec } \\
\text { GROUTH2O according to the above ratio. }\end{array}$ \\
\hline $\begin{array}{l}\text { Mercury } \\
\text { amalgamation / } \\
\text { HG-AMALG } \\
\text { (MIXER) }\end{array}$ & 68 & - & $\begin{array}{l}\text { Amalgamation of mercury wastes. Addition of } \\
\text { copper in ratio of } 0.7: 1 \text { (Cu:waste) as determined } \\
\text { by FORTRAN block ADDITIVE. }\end{array}$ \\
\hline $\begin{array}{l}\text { Metal } \\
\text { decontamination / } \\
\text { MET-DCON } \\
\text { (SEP) }\end{array}$ & 64 & - & $\begin{array}{l}\text { Decontamination of metal with abrasive blasting. } \\
\text { Assumes water input in a ratio of } 112 \mathrm{lb} \text { per } 468 \\
\mathrm{lb} \text { waste. Assumes all grit recycled (no make-up } \\
\text { grit added to process). Liquid stream }\left(\mathrm{H}_{2} \mathrm{O}, \mathrm{H}_{2} \text {, }\right. \\
\left.\mathrm{C}, \mathrm{Al}_{2} \mathrm{O}_{3} \text {, and } \mathrm{SiO}_{2}\right) \text { is sent to aqueous waste } \\
\text { treatment. Clean metal }(\mathrm{Fe}) \text { exits the process. }\end{array}$ \\
\hline $\begin{array}{l}\text { Organic } \\
\text { destruction feed } \\
\text { preparation / } \\
\text { MEO-MIXR } \\
\text { (MIXER) }\end{array}$ & 183 & - & $\begin{array}{l}\text { Mixes all feed material to MEO process, including } \\
\text { waste input, reagents, and recycle streams (water } \\
\text { and nitric acid). Make-up silver nitrate is } \\
\text { determined by FORTRAN block AGNO3-IN. } \\
\text { Make-up nitric acid is determined by FORTRAN } \\
\text { block HNO3-IN2. }\end{array}$ \\
\hline
\end{tabular}




\begin{tabular}{|c|c|c|c|}
\hline $\begin{array}{l}\text { MEO process - } \\
\text { anode / } \\
\text { MEO-ANOD } \\
\text { (RSTOIC) }\end{array}$ & 176 & 47505764 & $\begin{array}{l}\text { Reacts at } 80^{\circ} \mathrm{C} \text { and } 35 \text { psig. } \\
\text { Reaction } \\
\mathrm{C}+2 \mathrm{H}_{2} \mathrm{O} \rightarrow \mathrm{CO}_{2}+4 \mathrm{H}^{+}+4 \mathrm{e}^{-} \\
\mathrm{H}_{2} \rightarrow 2 \mathrm{H}^{+}+2 \mathrm{e}^{-} \\
\mathrm{Cl}_{2}+2 \mathrm{H}^{+}+2 \mathrm{e}^{-} \rightarrow 2 \mathrm{HCl} \\
2 \mathrm{~S}+2 \mathrm{H}_{2} \mathrm{O}+3 \mathrm{O}_{2} \rightarrow 2 \mathrm{H}_{2} \mathrm{SO}_{4} \\
2 \mathrm{~N}_{2}+2 \mathrm{H}_{2} \mathrm{O}+5 \mathrm{O}_{2} \rightarrow 4 \mathrm{HNO}_{3}\end{array}$ \\
\hline $\begin{array}{l}\text { MEO process - } \\
\text { cathode / } \\
\text { MEO-CATH } \\
\text { (RSTOIC) }\end{array}$ & 176 & -50040844 & $\begin{array}{l}\text { Reacts at } 80^{\circ} \mathrm{C} \text { and } 35 \text { psig at } 100 \% \text { conversion. } \\
\begin{array}{lc}\text { Reaction } & \text { Conversion } \\
\mathrm{HNO}_{3}+2 \mathrm{H}^{+}+2 \mathrm{e}^{-} \rightarrow \mathrm{H}_{2} \mathrm{O}+\mathrm{HNO}_{2} & 1.00 \\
\mathrm{AgNO}_{3}+\mathrm{HCl} \rightarrow \mathrm{AgCl}+\mathrm{HNO}_{3} & 0.99\end{array}\end{array}$ \\
\hline $\begin{array}{l}\text { Silver } \\
\text { precipitation / } \\
\text { AG-PPT } \\
\text { (RSTOIC) }\end{array}$ & 176 & 0 & $\begin{array}{l}\text { Precipitation of remaining } \mathrm{AgNO}_{3} \text { with addition } \\
\text { of } \mathrm{HCl} \text {. Reacts and } 80^{\circ} \mathrm{C} \text { and } 35 \text { psig at } 100 \% \\
\text { conversion. }\end{array}$ \\
\hline $\begin{array}{l}\text { MEO process / } \\
\text { MEO3 (RSTOIC) }\end{array}$ & 176 & -300 & $\begin{array}{l}\text { Converts remaining nitrate to } \mathrm{HNO}_{2} \text { at } 80^{\circ} \mathrm{C} \text { and } \\
35 \text { psig. } \\
\begin{array}{l}\text { Reaction } \\
\begin{array}{l}4 \mathrm{H}^{+}+2 \mathrm{NO}_{3}^{-}+2 \mathrm{e}^{-} \rightarrow \\
\quad \mathrm{HNO}_{3}+\mathrm{HNO}_{2}+\mathrm{H}_{2} \mathrm{O}\end{array} \\
\mathrm{H}++\mathrm{Cl}-\rightarrow \mathrm{HCl}\end{array}\end{array}$ \\
\hline $\begin{array}{l}\text { Centrifuge- } \\
\text { Evaporator / } \\
\text { MEO-SPLT } \\
\text { (SEP) }\end{array}$ & 176 & - & $\begin{array}{l}\text { Separates gases, solids, and recycle streams. } \\
\text { Some } \mathrm{H}_{2} \mathrm{O} \text { and all } \mathrm{HCl} \text { is recycled to the } \mathrm{MEO} \\
\text { process and is controlled by design spec } \mathrm{H} 2 \mathrm{O}-\mathrm{IN} \text {. } \\
\text { The remaining } \mathrm{H}_{2} \mathrm{O}, \mathrm{H}_{2}, \mathrm{Cl}_{2}, \mathrm{H}_{2} \mathrm{SO}_{4} \text {, and } \mathrm{HCl} \text { is } \\
\text { routed aqueous waste treatment. Gases }\left(\mathrm{O}_{2}, \mathrm{CO}_{2} \text {, }\right. \\
\left.\mathrm{CH}_{4} \text {, and } \mathrm{HNO}_{2}\right) \text { are routed to nitrous acid } \\
\text { conversion and } \mathrm{NO}_{\mathrm{x}} \text { removal. } \mathrm{AgCl}, \mathrm{AgNO}_{3} \text {, } \\
\mathrm{HNO}_{3}, \mathrm{NO}_{3^{-}}, \mathrm{Ag}^{+}, \mathrm{Cl} \text {, and e are routed to silver } \\
\text { conversion. } \mathrm{S}, \mathrm{F}_{2}, \mathrm{Ba}, \mathrm{Fe}, \mathrm{Cd}, \mathrm{Cr}, \mathrm{C}, \mathrm{SiO}_{2}, \mathrm{Al}_{2} \mathrm{O}_{3} \text {, } \\
\text { and } 10 \text { ppm of } \mathrm{HNO}_{3} \text { are routed to neutralization. }\end{array}$ \\
\hline
\end{tabular}




\begin{tabular}{|c|c|c|c|}
\hline $\begin{array}{l}\text { Nitrous acid } \\
\text { conversion / } \\
\text { FINO2RECV } \\
\text { (RSTOIC) }\end{array}$ & 302 & -735561 & $\begin{array}{l}\text { Converts } \mathrm{HNO}_{2} \text { to } \mathrm{HNO}_{3} \text { and to } \mathrm{NO} \text { at } 423 \mathrm{~K} \text { and } \\
35 \text { psig. } \mathrm{O}_{2} \text { input }(1 \% \text { excess) is determined by } \\
\text { design spec } \mathrm{O} 2-\mathrm{HNO} \text {. } \\
\begin{array}{lc}\text { Reaction } & \text { Conversion } \\
2 \mathrm{HNO}_{2}+\mathrm{O}_{2} \rightarrow 2 \mathrm{HNO}_{3} & 0.99 \\
4 \mathrm{HNO}_{2} \rightarrow 2 \mathrm{H}_{2} \mathrm{O}+4 \mathrm{NO}+\mathrm{O}_{2} & 0.01\end{array}\end{array}$ \\
\hline $\begin{array}{l}\text { Nitrous acid } \\
\text { conversion / } \\
\text { NOX-SPLT } \\
\text { (SEP) }\end{array}$ & 302 & - & $\begin{array}{l}\text { Separates } \mathrm{HNO}_{3} \text { for recycle back to MEO } \\
\text { reactor. }\end{array}$ \\
\hline $\begin{array}{l}\text { NOx removal / } \\
\text { NOX-CONV } \\
\text { (RSTOIC) }\end{array}$ & 68 & -78750 & $\begin{array}{l}\text { Reacts } \mathrm{NO} \text { at } 68^{\circ} \mathrm{F} \text { and } 1 \text { atm and } 99 \% \\
\text { conversion. Reaction is. Stoichiometric } \mathrm{NH}_{3} \\
\text { input is determined by FORTRAN block X-NH3. } \\
\text { Gases are then routed to APC. } \\
\begin{array}{l}\text { Reaction } \\
6 \mathrm{NO}+4 \mathrm{NH}_{3} \rightarrow 5 \mathrm{~N}_{2}+6 \mathrm{H}_{2} \mathrm{O} \quad \frac{\text { Conversion }}{0.99}\end{array}\end{array}$ \\
\hline $\begin{array}{l}\text { Silver conversion- } \\
\text { silver dissolving / } \\
\text { AGRECOVR } \\
\text { (RSTOIC) }\end{array}$ & 100 & 140361 & 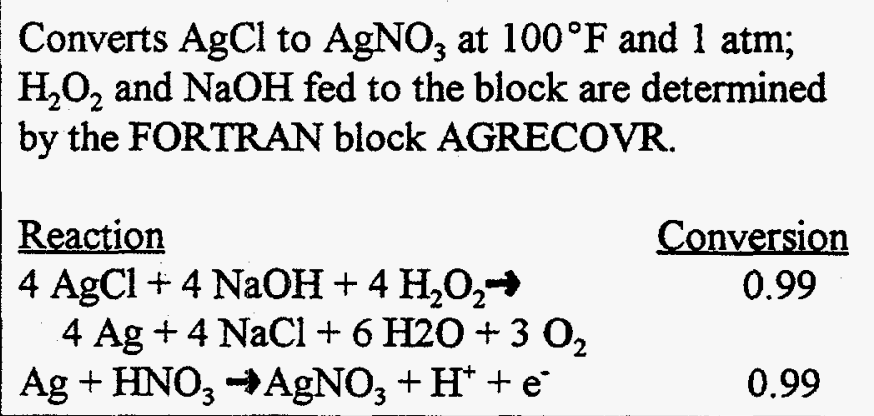 \\
\hline $\begin{array}{l}\text { Centrifuge / } \\
\text { AG-SPLT (SEP) }\end{array}$ & 100 & - & $\begin{array}{l}\text { Separates } \mathrm{AgNO}_{3} \text { and } \mathrm{HNO}_{3} \text { for recycle to MEO } \\
\text { reactor. } \mathrm{Gases}\left(\mathrm{O}_{2}\right) \text { are routed to } \mathrm{APC} \text { system. } \\
\text { Liquids }\left(\mathrm{H}_{2} \mathrm{O}, \mathrm{Ag}, \mathrm{NaOH}, \mathrm{NaCl}, \mathrm{H}^{+}, \mathrm{e}^{-}, \mathrm{H}_{2} \mathrm{O}_{2} \text {, }\right. \\
\mathrm{AgCl}) \text { are routed to aqueous waste treatment. }\end{array}$ \\
\hline $\begin{array}{l}\text { Neutralization / } \\
\text { NEUTRAL } \\
\text { (RSTOIC) }\end{array}$ & 68 & -265 & 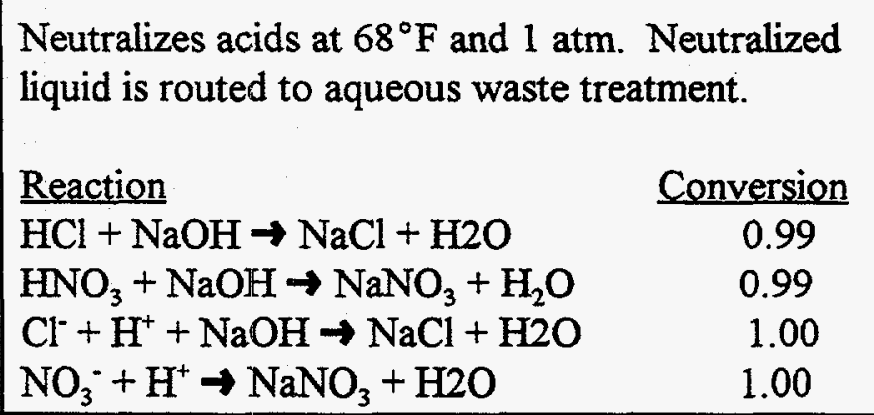 \\
\hline
\end{tabular}




\begin{tabular}{|c|c|c|c|}
\hline $\begin{array}{l}\text { Lead recovery- } \\
\text { separation / } \\
\text { PB-SPLIT (SEP) }\end{array}$ & 68 & - & $\begin{array}{l}\text { Separates brick or sheet lead (assumed to be } 81 \% \\
\text { of total) for decontamination. Remaining lead } \\
\text { (leaded gloves, blankets, shot) is routed to grout } \\
\text { stabilization. }\end{array}$ \\
\hline $\begin{array}{l}\text { Lead } \\
\text { decontamination / } \\
\text { PB-DECON } \\
\text { (SEP) }\end{array}$ & 68 & - & $\begin{array}{l}\text { Decontaminates lead with water wash, separates } \\
\text { clean lead, which exits the system. Liquid and } \\
\text { sludge are routed to process residue treatment } \\
\text { subsystem. }\end{array}$ \\
\hline $\begin{array}{l}\text { Polymer feed } \\
\text { preparation / } \\
\text { POLY-MIX } \\
\text { (MIXER) }\end{array}$ & 101 & - & Mixes incoming waste for polymer stabilization. \\
\hline $\begin{array}{l}\text { Dryer / DRYER } \\
\text { (HEATER) }\end{array}$ & 200 & -19532 & $\begin{array}{l}\text { Removes excess water prior to polymer } \\
\text { stabilization. }\end{array}$ \\
\hline $\begin{array}{l}\text { Dryer / } \\
\text { WATERSEP } \\
\text { (SEP) }\end{array}$ & 200 & - & $\begin{array}{l}\text { Separates water, input to polymer stabilization is } \\
\text { dried to } 5 \% \text { moisture content, as determined by } \\
\text { design spec DRYER. Excess } \mathrm{H}_{2} \mathrm{O}, \mathrm{NO} \text {, and } \mathrm{SO}_{2} \\
\text { are sent to aqueous waste treatment subsystem. }\end{array}$ \\
\hline $\begin{array}{l}\text { Mixing / } \\
\text { POLYSTAB } \\
\text { (MIXER) }\end{array}$ & & & $\begin{array}{l}\text { Adds polyethylene to encapsulate the waste. } \\
\text { Polymer:waste ratio is } 1: 1 \text {. }\end{array}$ \\
\hline $\begin{array}{l}\text { Process residue } \\
\text { dryer / } \\
\text { VACTRAX1 } \\
\text { (RGIBBS) }\end{array}$ & 550 & 540225 & $\begin{array}{l}\text { Models the vacuum desorber for process residue } \\
\text { and inorganic sludges. Operates at } 550^{\circ} \mathrm{F} \text { and } 1 \\
\text { psig. Equilibrium is assumed for species entered } \\
\text { in the RGIBBS block: } \mathrm{H}_{2} \mathrm{SO}_{4} \text { and } \mathrm{CH}_{4} \text { are } \\
\text { assumed to form from feed components. } \mathrm{O}_{2} \text { is } \\
\text { excluded from reaction. A nitrogen sweep gas is } \\
\text { used at a rate of } 1.09 \mathrm{ft}^{3} \text { per lb waste }(0.0793 \mathrm{lb} \\
\mathrm{N}_{2} / \mathrm{b} \text { waste) as determined by FORTRAN block } \\
\text { N2SWEEP. }\end{array}$ \\
\hline
\end{tabular}




\begin{tabular}{|c|c|c|c|}
\hline $\begin{array}{l}\text { Mercury leaching } \\
\text { / VACSEP-1 } \\
\text { (SEP) }\end{array}$ & 97 & - & $\begin{array}{l}\text { Mercury leaching of process residue and inorganic } \\
\text { sludges by addition of leach water ( } 30 \% \text { of mass } \\
\text { of incoming waste). Also includes separation of } \\
\text { gases, liquids, organics, } \mathrm{Hg} \text {-contaminated liquids, } \\
\text { and solids. } \\
\text { Gases }\left(\mathrm{N}_{2} \text { ) are sent to APC subsystem. Solids }\right. \\
\left(\mathrm{H}_{2} \mathrm{O} \text { in leach water, } 50 \% \text { of } \mathrm{H}_{2}, \mathrm{O}_{2}, \mathrm{~S}, 50 \% \text { of C, }\right. \\
\mathrm{SiO}_{2}, \mathrm{Al}_{2} \mathrm{O}_{3}, \mathrm{Ba}, \mathrm{As}, \mathrm{Pb}, \mathrm{Fe}, \mathrm{Cd}, \mathrm{Cr}, \mathrm{Se}, \mathrm{Ag} \text { ) are } \\
\text { routed to grout stabilization subsystem. Liquids } \\
\text { (balance of } \mathrm{H}_{2} \mathrm{O}, \mathrm{H}_{2} \mathrm{SO}_{4} \text { ) are sent to aqueous } \\
\text { waste treatment. Organics ( } 50 \% \text { of } \mathrm{H}_{2}, 50 \% \text { of } \\
\mathrm{C}, 50 \% \text { of } \mathrm{Cl}_{2} \text { ) are routed to the organic } \\
\text { destruction subsystem. Mercury separated is } \\
\text { routed to mercury amalgamation. }\end{array}$ \\
\hline Special waste & 68 & - & $\begin{array}{l}\text { As with the ITTS study, no model was } \\
\text { constructed for special waste. }\end{array}$ \\
\hline
\end{tabular}




\section{System 2 - Desorption Case}

\begin{tabular}{|c|c|c|c|}
\hline \multicolumn{4}{|c|}{ ASSUMPTIONS and ENERGY BALANCES for DESORPTION CASE, System 2} \\
\hline \multicolumn{4}{|c|}{ GENERAL COMMENTS: } \\
\hline $\begin{array}{l}\text { Unit Operation / } \\
\text { ASPEN PLUS } \\
\text { Block Name }\end{array}$ & $\begin{array}{l}\text { Outle } \\
t \\
\text { Temp } \\
\left({ }^{\circ} \mathrm{F}\right) \\
\end{array}$ & $\begin{array}{l}\text { Heat Duty } \\
\text { (MMBtu/hr) }\end{array}$ & Description and Assumptions \\
\hline $\begin{array}{l}\text { APC filter / } \\
\text { FULTER (SEP) }\end{array}$ & 251 & - & $\begin{array}{l}\text { Filter to separate heavy metals and other solids } \\
\text { from gas stream. Gas streams entering the APC } \\
\text { system usually have all particulates removed } \\
\text { beforehand. }\end{array}$ \\
\hline $\begin{array}{l}\text { Gas phase corona } \\
\text { reactor / } \\
\text { GPCR (RSTOIC) }\end{array}$ & 378 & 24416 & 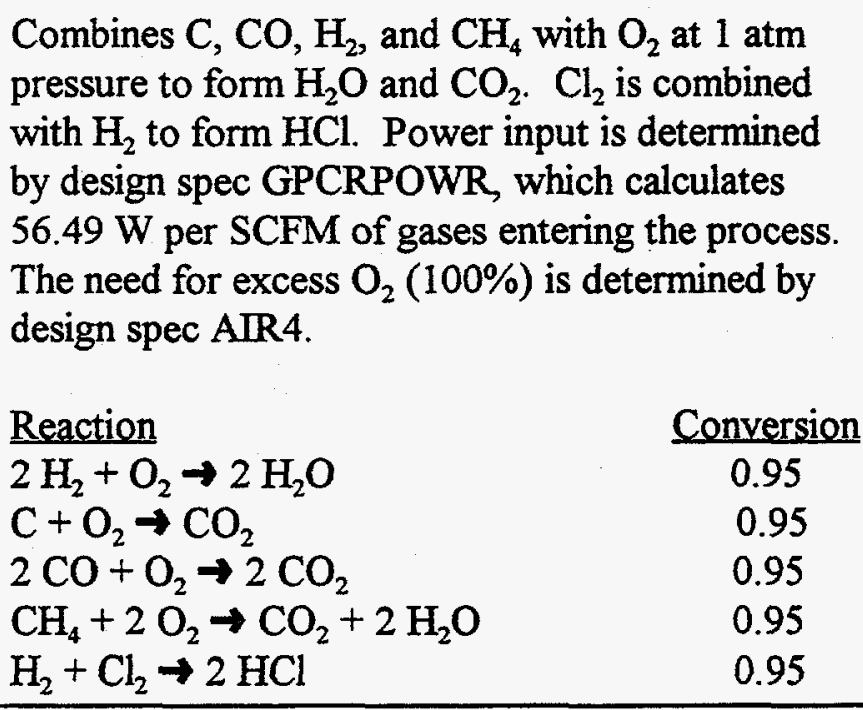 \\
\hline $\begin{array}{l}\text { Acid gas scrubber } \\
\text { / NEUTRAL3 } \\
\text { (RSTOIC) }\end{array}$ & 100 & -52239 & $\begin{array}{l}\text { Neutralizes } \mathrm{HCl}, \mathrm{Cl}_{2} \text {, and } \mathrm{SO}_{2} \text { with } \mathrm{NaOH} \text { at } 1 \text { atm } \\
\text { pressure and } 100^{\circ} \mathrm{F} \text { to form } \mathrm{NaCl} \text { and } \mathrm{Na}_{2} \mathrm{SO}_{4} \text {. } \\
\text { Excess } \mathrm{NaOH}(1 \%) \text { is determined by design spec } \\
\text { NAOH-3. } \\
\begin{array}{ll}\text { Reaction } & \text { Conversion } \\
\mathrm{HCl}+\mathrm{NaOH} \rightarrow \mathrm{NaCl}+\mathrm{H}_{2} \mathrm{O} & 0.99 \\
2 \mathrm{Cl}_{2}+4 \mathrm{NaOH} \rightarrow 4 \mathrm{NaCl}+2 \mathrm{H}_{2} \mathrm{O}+\mathrm{O}_{2} & 0.99 \\
2 \mathrm{SO}_{2}+4 \mathrm{NaOH}+\mathrm{O}_{2} \rightarrow 2 \mathrm{Na}_{2} \mathrm{SO}_{4}+2 \mathrm{H}_{2} \mathrm{O} & 0.99\end{array}\end{array}$ \\
\hline
\end{tabular}




\begin{tabular}{|c|c|c|c|}
\hline $\begin{array}{l}\text { Scrubber liquor } \\
\text { sump / } \\
\text { BLOWDOWN } \\
\text { (SEP) }\end{array}$ & 100 & - & $\begin{array}{l}\text { Separates salts formed in scrubber. } \mathrm{NaCl}, \mathrm{NaOH} \text {, } \\
\text { and } \mathrm{Na}_{2} \mathrm{SO}_{4} \text { are sent with some water to aqueous } \\
\text { waste treatment subsystem. Water flow rate } \\
\text { calculated for } 20 \% \text { salt solution by design spec } \\
\text { SALTSOLN. }\end{array}$ \\
\hline $\begin{array}{l}\text { Mist eliminator/ } \\
\text { FLASH } \\
\text { (FLASH2) }\end{array}$ & 100 & 0 & $\begin{array}{l}\text { Two-phase flash to separate out additional } \\
\text { moisture. Flash determines vapor-liquid } \\
\text { equilibrium at } 100^{\circ} \mathrm{F} \text { and } 1 \text { atm. Moisture sent to } \\
\text { aqueous waste treatment subsystem. }\end{array}$ \\
\hline $\begin{array}{l}\text { Filter / } \\
\text { HETRHEPA } \\
\text { (HEATER) }\end{array}$ & 300 & 37124 & $\begin{array}{l}\text { Heater to raise temperature of gas stream to } 300^{\circ} \mathrm{F} \\
\text { to avoid condensate in the HEPA filter. }\end{array}$ \\
\hline $\begin{array}{l}\text { Ozone generator / } \\
\text { OZ-GEN } \\
\text { (RSTOIC) }\end{array}$ & 100 & 1162 & $\begin{array}{l}\mathrm{O}_{3} \text { generated from } \mathrm{O}_{2} \text { at } 100^{\circ} \mathrm{F} \text { and } 1 \mathrm{~atm} . \\
\frac{\text { Reaction }}{3 \mathrm{O}_{2} \rightarrow 2 \mathrm{O}_{3}} \\
\end{array}$ \\
\hline $\begin{array}{l}\text { Aqueous liquids } \\
\text { sorting / } \\
\text { PHOTOMIX } \\
\text { (MIXER) }\end{array}$ & 77 & - & $\begin{array}{l}\text { Combines all streams entering aqueous waste } \\
\text { treatment subsystem. }\end{array}$ \\
\hline $\begin{array}{l}\text { Gross organics } \\
\text { removal / OILSEP } \\
\text { (SEP) }\end{array}$ & 77 & - & $\begin{array}{l}\text { Oil-water separator to remove } \mathrm{C} \text { and } \mathrm{H}_{2} \text { from } \\
\text { liquid stream at an assumed efficiency of } 99 \% \text {. }\end{array}$ \\
\hline $\begin{array}{l}\text { OH generator / } \\
\text { OH-GEN } \\
\text { (RSTOIC) }\end{array}$ & 100 & 11659 & $\begin{array}{l}\text { Generates } \mathrm{OH} \text { radicals from } \mathrm{H}_{2} \mathrm{O}_{2} \text { and } \mathrm{O}_{3} \text {. } \\
\begin{array}{lc}\text { Reaction } & \text { Conversion } \\
\mathrm{H}_{2} \mathrm{O}_{2} \rightarrow 2 \mathrm{OH} \bullet & 0.99 \\
\mathrm{O}_{3}+3 \mathrm{H}_{2} \mathrm{O} \rightarrow 6 \mathrm{OH} \cdot & 0.99 \\
\end{array}\end{array}$ \\
\hline $\begin{array}{l}\text { UV reactor / } \\
\text { PHOTO-UV } \\
\text { (RSTOIC) }\end{array}$ & 100 & -18198 & $\begin{array}{l}\text { Combines } \mathrm{C}, \mathrm{H}_{2} \text { with } \mathrm{OH} \text { radicals. } \\
\text { Reaction } \\
\mathrm{C}+4 \mathrm{OH} \cdot \rightarrow \mathrm{CO}_{2}+2 \mathrm{H} 2 \mathrm{O} \\
\mathrm{H}_{2}+4 \mathrm{OH} \cdot \rightarrow 2 \mathrm{H} 2 \mathrm{O} \\
\mathrm{H}_{2}+\mathrm{Cl}_{2} \rightarrow 2 \mathrm{HCl}\end{array}$ \\
\hline
\end{tabular}




\begin{tabular}{|c|c|c|c|}
\hline $\begin{array}{l}\text { Ozone } \\
\text { decomposition / } \\
\text { OZ-DECOM } \\
\text { (RSTOIC) }\end{array}$ & 100 & -91558 & $\begin{array}{l}\text { Decomposes unreacted ozone and neutralizes acids } \\
\text { at } 100^{\circ} \mathrm{F} \text { and } 1 \text { atm. Excess } \mathrm{NaOH}(1 \%) \text { is } \\
\text { determined by design spec XX-NAOH. } \\
\begin{array}{lc}\text { Reaction } & \text { Conversion } \\
2 \mathrm{O}_{3} \rightarrow 3 \mathrm{O}_{2} & 0.999 \\
\mathrm{NaOH}+\mathrm{HCl} \rightarrow \mathrm{NaCl}+\mathrm{H}_{2} \mathrm{O} & 0.99 \\
4 \mathrm{NaOH}+2 \mathrm{SO}_{2}+\mathrm{O}_{2} \rightarrow 2 \mathrm{Na}_{2} \mathrm{SO}_{4}+2 \mathrm{H}_{2} \mathrm{O} & 0.99 \\
4 \mathrm{NaOH}+2 \mathrm{Cl}_{2} \rightarrow 4 \mathrm{NaCl}_{2} \mathrm{H}_{2} \mathrm{O}+\mathrm{O}_{2} & 0.99 \\
2 \mathrm{NaOH}+\mathrm{H}_{2} \mathrm{SO}_{4} \rightarrow \mathrm{Na}_{2} \mathrm{SO}_{4}+2 \mathrm{H}_{2} \mathrm{O} & 0.99 \\
\end{array}\end{array}$ \\
\hline $\begin{array}{l}\text { Gas, water, sludge } \\
\text { separator / AQ- } \\
\text { SPLIT (SEP) }\end{array}$ & 100 & - & $\begin{array}{l}\text { Separates gas, liquid, and sludge from aqueous } \\
\text { waste treatment. Gases }\left(\mathrm{O}_{2}, \mathrm{H}_{2}, \mathrm{~N}_{2}, \mathrm{NO}, \mathrm{CO}_{2}, \mathrm{O}_{3}\right) \\
\text { are sent to } \mathrm{APC} \text { subsystem. Liquids }\left(\mathrm{H}_{2} \mathrm{O}, \mathrm{SO}_{2} \text {, }\right. \\
\mathrm{H}_{2} \mathrm{SO}_{4}, \mathrm{HCl}, \mathrm{H}_{2} \mathrm{O}_{2}, \mathrm{OH} \text { radicals, } 1 \% \text { of C, } 1 \% \text { of } \\
\left.\mathrm{SiO}_{2}, 1 \% \text { of } \mathrm{Al}_{2} \mathrm{O}_{3}\right) \text { are available for recycle in the } \\
\text { system. Solids }(\mathrm{As}, \mathrm{Ba}, \mathrm{Cd}, \mathrm{Cr}, \mathrm{Pb}, \mathrm{Se}, \mathrm{Ag}, \mathrm{NaCl} \text {, } \\
\mathrm{FeCl}_{3}, \mathrm{AgCl}, \mathrm{NaOH}, \mathrm{Na}_{2} \mathrm{SO}_{4}, 99 \% \text { of } \mathrm{C}, 99 \% \text { of } \\
\left.\mathrm{SiO}_{2}, 99 \% \text { of } \mathrm{Al}_{2} \mathrm{O}_{3}\right) \text { are routed to polymer } \\
\text { stabilization subsystem. }\end{array}$ \\
\hline $\begin{array}{l}\text { Evaporator / } \\
\text { CONCENT8 } \\
\text { (FLASH2) }\end{array}$ & 150 & 43 & $\begin{array}{l}\text { Models evaporation of scrubber liquor from APC } \\
\text { subsystem. Two-phase flash determines vapor- } \\
\text { liquid equilibrium at } 150^{\circ} \mathrm{F} \text { and } 1 \mathrm{~atm} \text {. Vapor sent } \\
\text { to PHOTOMIX, liquid/solid phase sent to polymer } \\
\text { stabilization subsystem. }\end{array}$ \\
\hline $\begin{array}{l}\text { Soil dryer / } \\
\text { VACTRAX3 } \\
\text { (RGIBBS) }\end{array}$ & 550 & 54911 & $\begin{array}{l}\text { Models the vacuum desorber for bulk soils. } \\
\text { Operates at } 550^{\circ} \mathrm{F} \text { and } 1 \text { psig. Equilibrium is } \\
\text { assumed for species entered in the RGIBBS block: } \\
\mathrm{HCl} \text { and } \mathrm{CH}_{4} \text { are assumed to form from feed } \\
\text { components. } \mathrm{O}_{2} \text { is excluded from reaction. A } \\
\text { nitrogen sweep gas is used at a rate of } 1.09 \mathrm{f}^{3} \text { per } \\
\text { lb waste }(0.0793 \mathrm{lb} \mathrm{N} / \mathrm{lb} \text { waste) as determined by } \\
\text { FORTRAN block N2SWEEP. }\end{array}$ \\
\hline
\end{tabular}




\begin{tabular}{|c|c|c|c|}
\hline $\begin{array}{l}\text { Mercury leaching / } \\
\text { VACSEP-3 (SEP) }\end{array}$ & 91 & - & $\begin{array}{l}\text { Mercury leaching of soils by addition of leach } \\
\text { water ( } 30 \% \text { of mass of incoming waste). Also } \\
\text { includes separation of gases, liquids, organics, } \mathrm{Hg} \text { - } \\
\text { contaminated liquids, and solids. } \\
\text { Gases }\left(\mathrm{N}_{2}\right) \text { are sent to APC subsystem. Solids } \\
\left(\mathrm{H}_{2} \mathrm{O} \text { in leach water, } 50 \% \text { of } \mathrm{H}_{2}, 50 \% \text { of } \mathrm{C}, \mathrm{SiO}_{2} \text {, }\right. \\
\left.\mathrm{Al}_{2} \mathrm{O}_{3}\right) \text { are routed to grout stabilization subsystem. } \\
\left.\mathrm{Liquids} \text { (balance of } \mathrm{H}_{2} \mathrm{O}, \mathrm{HCl}\right) \text { are sent to aqueous } \\
\text { waste treatment. Organics }\left(50 \% \text { of } \mathrm{H}_{2}, \mathrm{CH}_{4}, 50 \%\right. \\
\text { of } \mathrm{C} \text { ) are routed to the organic destruction } \\
\text { subsystem. }\end{array}$ \\
\hline $\begin{array}{l}\text { Grout stabilization } \\
\text { feed preparation / } \\
\text { GROUTMIX } \\
\text { (MIXER) }\end{array}$ & 71 & - & Combines all waste feed to grout stabilization. \\
\hline $\begin{array}{l}\text { Microsolidification } \\
\text { / GROUTER } \\
\text { (MIXER) }\end{array}$ & 69 & - & $\begin{array}{l}\text { Combines cement and water with incoming waste } \\
\text { feed. Amount of cement is determined by design } \\
\text { spec GROUT. Total grout:waste ratio is } 2: 1 \text { and } \\
\text { dry grout:water ratio is } 0.7: 0.3 \text {. Amount of water } \\
\text { is determined by design spec GROUTH } 20 \\
\text { according to the above ratio. }\end{array}$ \\
\hline $\begin{array}{l}\text { Mercury } \\
\text { amalgamation / } \\
\text { HG-AMALG } \\
\text { (MIXER) }\end{array}$ & 68 & - & $\begin{array}{l}\text { Amalgamation of mercury wastes. Addition of } \\
\text { copper in ratio of } 0.7: 1 \text { (Cu:waste) as determined } \\
\text { by FORTRAN block ADDITIVE. }\end{array}$ \\
\hline $\begin{array}{l}\text { Metal } \\
\text { decontamination / } \\
\text { MET-DCON } \\
\text { (SEP) }\end{array}$ & 64 & - & $\begin{array}{l}\text { Decontamination of metal with abrasive blasting. } \\
\text { Assumes water input in a ratio of } 112 \mathrm{lb} \text { per } 468 \mathrm{lb} \\
\text { waste. Assumes all grit recycled (no make-up grit } \\
\text { added to process). Liquid stream }\left(\mathrm{H}_{2} \mathrm{O}, \mathrm{H}_{2}, \mathrm{C} \text {, }\right. \\
\mathrm{Al}_{2} \mathrm{O}_{3} \text {, and } \mathrm{SiO}_{2} \text { ) is sent to aqueous waste } \\
\text { treatment. Clean metal }(\mathrm{Fe} \text { ) exits the process. }\end{array}$ \\
\hline
\end{tabular}




\begin{tabular}{|c|c|c|c|}
\hline $\begin{array}{l}\text { Organic } \\
\text { destruction / } \\
\text { CWO-1 } \\
\text { (RSTOIC) }\end{array}$ & 302 & -3724699 & 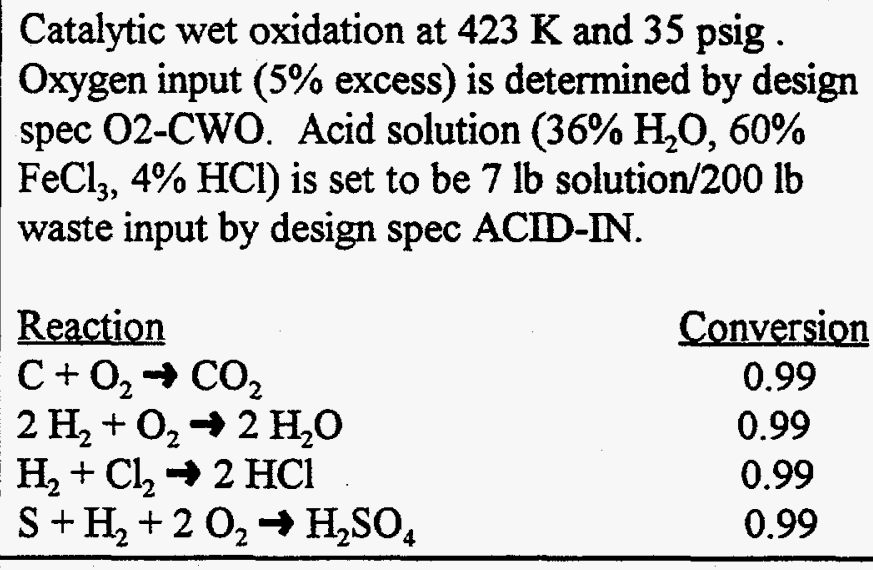 \\
\hline $\begin{array}{l}\text { Organic } \\
\text { destruction filter / } \\
\text { CWOSPLT (SEP) }\end{array}$ & 299 & - & $\begin{array}{l}\text { Separation of products from CWO reactor. Solids } \\
\left(\mathrm{S}, \mathrm{Fe}, \mathrm{Ba}, \mathrm{Cd}, \mathrm{Cr}, 50 \% \text { of } \mathrm{Fe}(\mathrm{OH})_{3}, \mathrm{C}, \mathrm{SiO}_{2}\right. \\
\left.\mathrm{Al}_{2} \mathrm{O}_{3}\right) \text { are sent to polymer stabilization. Liquids } \\
\left(\mathrm{H}_{2} \mathrm{O}, \mathrm{HCl}, \mathrm{NaCl}, \mathrm{FeCl}_{3}, \mathrm{H}_{2} \mathrm{SO}_{4} \text {, and } 50 \% \text { of }\right. \\
\left.\mathrm{Fe}(\mathrm{OH})_{3}\right) \text { are sent for neutralization. Gases }\left(\mathrm{O}_{2} \text {, }\right. \\
\left.\mathrm{Cl}_{2}, \mathrm{~F}_{2}, \mathrm{CO}_{2}, \mathrm{CH}_{4}\right) \text { are subject to equilibration and } \\
\text { then sent to the APC subsystem. }\end{array}$ \\
\hline $\begin{array}{l}\text { Gas equilibration / } \\
\text { EQUIL-1 } \\
\text { (RGIBBS) }\end{array}$ & 302 & -7324 & $\begin{array}{l}\text { This block equilibrates the gas leaving the } \mathrm{CWO} \\
\text { system to account for the non-equilibrium of the } \\
\mathrm{CWO} \text { reactor. It reacts the small amount of } \mathrm{CH}_{4} \\
\text { remaining with } \mathrm{O}_{2} \text { to form } \mathrm{H}_{2} \mathrm{O} \text { and } \mathrm{CO}_{2} . \mathrm{Cl}_{2} \text { is } \\
\text { combined with } \mathrm{H}_{2} \text { to form } \mathrm{HCl} \text {. }\end{array}$ \\
\hline $\begin{array}{l}\text { Neutralization / } \\
\text { NEUTRAL1 } \\
\text { (RSTOIC) }\end{array}$ & 100 & -364010 & 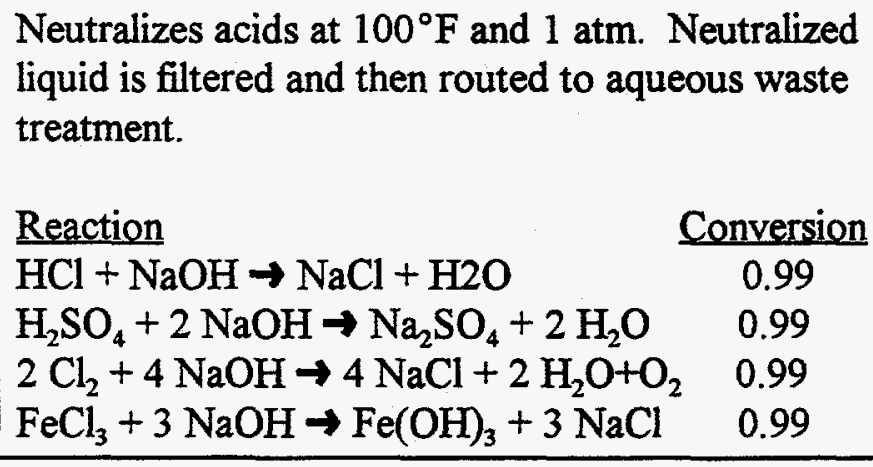 \\
\hline $\begin{array}{l}\text { Precipitation filter } \\
\text { / SALTFLTR }\end{array}$ & 100 & - & $\begin{array}{l}\text { Removes } \mathrm{Fe}(\mathrm{OH})_{3} \text { from aqueous stream. Solid is } \\
\text { routed to polymer stabilization. }\end{array}$ \\
\hline $\begin{array}{l}\text { Lead recovery- } \\
\text { separation / } \\
\text { PB-SPLIT (SEP) }\end{array}$ & 68 & - & $\begin{array}{l}\text { Separates brick or sheet lead (assumed to be } 81 \% \\
\text { of total) for decontamination. Remaining lead } \\
\text { (leaded gloves, blankets, shot) is routed to grout } \\
\text { stabilization. }\end{array}$ \\
\hline
\end{tabular}




\begin{tabular}{|c|c|c|c|}
\hline $\begin{array}{l}\text { Lead } \\
\text { decontamination / } \\
\text { PB-DECON } \\
\text { (SEP) }\end{array}$ & 63 & - & $\begin{array}{l}\text { Decontaminates lead with water wash, separates } \\
\text { clean lead, which exits the system. Liquid and } \\
\text { sludge are routed to process residue treatment } \\
\text { subsystem. }\end{array}$ \\
\hline $\begin{array}{l}\text { Polymer feed } \\
\text { preparation / } \\
\text { POLY-MIX } \\
\text { (MIXER) }\end{array}$ & 73 & - & Mixes incoming waste for polymer stabilization. \\
\hline $\begin{array}{l}\text { Dryer / DRYER } \\
\text { (HEATER) }\end{array}$ & 200 & 79322 & $\begin{array}{l}\text { Removes excess water prior to polymer } \\
\text { stabilization. }\end{array}$ \\
\hline $\begin{array}{l}\text { Dryer / } \\
\text { WATERSEP } \\
\text { (SEP) }\end{array}$ & 200 & - & $\begin{array}{l}\text { Separates water, input to polymer stabilization is } \\
\text { dried to } 5 \% \text { moisture content, as determined by } \\
\text { design spec DRYER. Excess } \mathrm{H}_{2} \mathrm{O}, \mathrm{NO} \text {, and } \mathrm{SO}_{2} \\
\text { are sent to aqueous waste treatment subsystem. }\end{array}$ \\
\hline $\begin{array}{l}\text { Mixing / } \\
\text { POLYSTAB } \\
\text { (MIXER) }\end{array}$ & 102 & - & $\begin{array}{l}\text { Adds polyethylene to encapsulate the waste. } \\
\text { Polymer:waste ratio is } 1: 1 \text {. }\end{array}$ \\
\hline $\begin{array}{l}\text { Process residue } \\
\text { dryer / } \\
\text { VACTRAX1 } \\
\text { (RGIBBS) }\end{array}$ & 550 & 515629 & $\begin{array}{l}\text { Models the vacuum desorber for process residue } \\
\text { and inorganic sludges. Operates at } 550^{\circ} \mathrm{F} \text { and } 1 \\
\text { psig. Equilibrium is assumed for species entered in } \\
\text { the RGIBBS block: } \mathrm{H}_{2} \mathrm{SO}_{4}, \mathrm{HCl} \text {, and } \mathrm{CH}_{4} \text { are } \\
\text { assumed to form from feed components. } \mathrm{O}_{2} \text { is } \\
\text { excluded from reaction. A nitrogen } 5 w e e p \text { gas is } \\
\text { used at a rate of } 1.09 \mathrm{ft}^{3} \text { per lb waste }(0.0793 \mathrm{lb} \\
\mathrm{N}_{2} / \mathrm{b} \text { waste) as determined by FORTRAN block } \\
\text { N2SWEEP. }\end{array}$ \\
\hline
\end{tabular}




\begin{tabular}{|c|c|c|c|}
\hline $\begin{array}{l}\text { Mercury leaching / } \\
\text { VACSEP-1 (SEP) }\end{array}$ & 68 & - & $\begin{array}{l}\text { Mercury leaching of process residue and inorganic } \\
\text { sludges by addition of leach water ( } 30 \% \text { of mass of } \\
\text { incoming waste). Also includes separation of } \\
\text { gases, liquids, organics, } \mathrm{Hg} \text {-contaminated liquids, } \\
\text { and solids. } \\
\\
\text { Gases }\left(\mathrm{N}_{2}\right) \text { are sent to } \mathrm{APC} \text { subsystem. Solids } \\
\left(\mathrm{H}_{2} \mathrm{O} \text { in leach water, } \mathrm{S}, 50 \% \text { of } \mathrm{H}_{2}, \mathrm{O}_{2}, 50 \% \text { of C, }\right. \\
\mathrm{SiO}_{2}, \mathrm{Al}_{2} \mathrm{O}_{3}, \mathrm{Ba}, \mathrm{As}, \mathrm{Pb}, \mathrm{Fe}, \mathrm{Cd}, \mathrm{Cr}, \mathrm{Se}, \mathrm{Ag} \text { ) are } \\
\text { routed to polymer stabilization subsystem. Liquids } \\
\left.\text { (balance of } \mathrm{H}_{2} \mathrm{O}, \mathrm{HCl}, \mathrm{H}_{2} \mathrm{SO} \mathrm{S}_{4}\right) \text { are sent to aqueous } \\
\text { waste treatment. Organics }\left(50 \% \text { of } \mathrm{H}_{2}, 50 \% \text { of C, }\right. \\
\left.\mathrm{CH}_{4}\right) \text { are routed to the organic destruction } \\
\text { subsystem. Mercury separated is routed to } \\
\text { mercury amalgamation. }\end{array}$ \\
\hline $\begin{array}{l}\text { Soft, open, } \\
\text { complex debris } \\
\text { dryer / } \\
\text { VACTRAX2 } \\
\text { (RGIBBS) }\end{array}$ & 250 & 518600 & $\begin{array}{l}\text { Models the vacuum desorber for soft, open, and } \\
\text { complex debris. Operates at } 250^{\circ} \mathrm{F} \text { and } 1 \text { psig. } \\
\text { Equilibrium is assumed for species entered in the } \\
\text { RGIBBS block: } \mathrm{HCl} \text { is assumed to form from feed } \\
\text { components. } \mathrm{O}_{2} \text { and } \mathrm{H}_{2} \mathrm{O} \text { are excluded from } \\
\text { reaction. A nitrogen sweep gas is used at a rate of } \\
1.09 \mathrm{ft}^{3} \text { per lb waste }(0.0793 \mathrm{lb} \mathrm{N} / \mathrm{lb} \text { waste) as } \\
\text { determined by FORTRAN block N2SWEEP. }\end{array}$ \\
\hline $\begin{array}{l}\text { Mercury leaching / } \\
\text { VACSEP-2 (SEP) }\end{array}$ & 68 & - & $\begin{array}{l}\text { Mercury leaching of soft, open, and complex } \\
\text { debris. Addition of leach water ( } 30 \% \text { of mass of } \\
\text { incoming waste). Also includes separation of } \\
\text { gases, liquids, organics, Hg-contaminated liquids, } \\
\text { and solids. } \\
\text { Gases }\left(\mathrm{N}_{2}\right) \text { are sent to APC subsystem. Solids } \\
\left(\mathrm{H}_{2} \mathrm{O} \text { in leach water, } \mathrm{S}, 90 \% \text { of } \mathrm{H}_{2}, \mathrm{O}_{2}, 90 \% \text { of } \mathrm{C} \text {, }\right. \\
\left.90 \% \text { of } \mathrm{F}_{2}, 90 \% \text { of } \mathrm{Cl}, \mathrm{SiO}_{2}, \mathrm{Al}_{2} \mathrm{O}_{3}, \mathrm{Fe}\right) \text { are routed } \\
\text { to grout stabilization subsystem. Liquids (balance } \\
\left.\text { of } \mathrm{H}_{2} \mathrm{O}, \mathrm{HCl}\right) \text { are sent to aqueous waste treatment. } \\
\text { Organics }\left(10 \% \text { of } \mathrm{H}_{2}, 10 \% \text { of C, } 10 \% \text { of } \mathrm{F}_{2}, 10 \%\right. \\
\text { of } \mathrm{Cl}_{2} \text {, and } \mathrm{SiO}_{2}, \text { ) are routed to the organic } \\
\text { destruction subsystem. Mercury separated is } \\
\text { routed to mercury amalgamation. }\end{array}$ \\
\hline Special waste & 68 & - & $\begin{array}{l}\text { As with the ITTS study, no model was constructed } \\
\text { for special waste. }\end{array}$ \\
\hline
\end{tabular}




\section{System 3 - Wash Case}

\begin{tabular}{|c|c|c|c|}
\hline \multicolumn{4}{|c|}{ ASSUMPTIONS and ENERGY BALANCES for WASH CASE, System 3} \\
\hline \multicolumn{4}{|c|}{ GENERAL COMMENTS: } \\
\hline $\begin{array}{l}\text { Unit Operation / } \\
\text { ASPEN PLUS } \\
\text { Block Name }\end{array}$ & $\begin{array}{l}\text { Outlet } \\
\text { Temp. } \\
\left({ }^{\circ} \mathrm{F}\right) \\
\end{array}$ & $\begin{array}{l}\text { Heat Duty } \\
(\mathrm{MMBtu} / \mathrm{hr})\end{array}$ & Description and Assumptions \\
\hline $\begin{array}{l}\text { APC filter / } \\
\text { FILTER (SEP) }\end{array}$ & 67 & - & $\begin{array}{l}\text { Filter to separate heavy metals and other solids } \\
\text { from gas stream. Gas streams entering the APC } \\
\text { system usually have all particulates removed } \\
\text { beforehand. }\end{array}$ \\
\hline $\begin{array}{l}\text { Gas phase corona } \\
\text { reactor / } \\
\text { GPCR (RSTOIC) }\end{array}$ & 165 & 22933 & 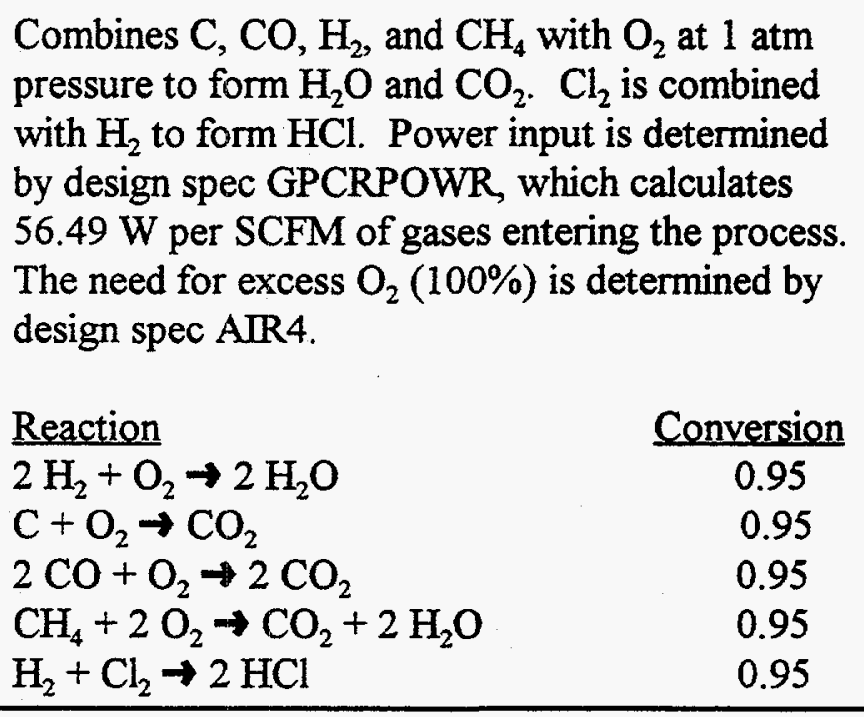 \\
\hline $\begin{array}{l}\text { Acid gas scrubber } \\
\text { / NEUTRAL3 } \\
\text { (RSTOIC) }\end{array}$ & 100 & -15633 & $\begin{array}{l}\text { Neutralizes } \mathrm{SO}_{2} \text { with } \mathrm{NaOH} \text { at } 1 \text { atm pressure and } \\
100^{\circ} \mathrm{F} \text { to form } \mathrm{Na}_{2} \mathrm{SO}_{4} \text { at a conversion level of } \\
99 \% \text {. Excess } \mathrm{NaOH}(1 \%) \text { is determined by } \\
\text { design spec } \mathrm{NAOH}-3 \text {. } \\
\text { Reaction } \\
2 \mathrm{SO}_{2}+4 \mathrm{NaOH}+\mathrm{O}_{2} \rightarrow 2 \mathrm{Na}_{2} \mathrm{SO}_{4}+2 \mathrm{H}_{2} \mathrm{O} 0.99\end{array}$ \\
\hline $\begin{array}{l}\text { Scrubber liquor } \\
\text { sump / } \\
\text { BLOWDOWN } \\
\text { (SEP) }\end{array}$ & 100 & - & $\begin{array}{l}\text { Separates salts formed in scrubber. } \mathrm{Na}_{2} \mathrm{SO}_{4} \text { and } \\
\mathrm{NaOH} \text { are sent with some water to aqueous waste } \\
\text { treatment subsystem. Water flow rate calculated } \\
\text { for } 20 \% \text { salt solution by design spec SALTSOLN. }\end{array}$ \\
\hline
\end{tabular}




\begin{tabular}{|c|c|c|c|}
\hline $\begin{array}{l}\text { Mist eliminator / } \\
\text { FLASH } \\
\text { (FLASH2) }\end{array}$ & 100 & 0 & $\begin{array}{l}\text { Two-phase flash to separate out additional } \\
\text { moisture. Flash determines vapor-liquid } \\
\text { equilibrium at } 100^{\circ} \mathrm{F} \text { and } 1 \text { atm. Moisture sent to } \\
\text { aqueous waste treatment subsystem. }\end{array}$ \\
\hline $\begin{array}{l}\text { Filter / } \\
\text { HETRHEPA } \\
\text { (HEATER) }\end{array}$ & 300 & 37070 & $\begin{array}{l}\text { Heater to raise temperature of gas stream to } \\
300^{\circ} \mathrm{F} \text { to avoid condensate in HEPA filter. }\end{array}$ \\
\hline $\begin{array}{l}\text { Ozone generator / } \\
\text { OZ-GEN } \\
\text { (RSTOIC) }\end{array}$ & 100 & 2621 & $\begin{array}{l}\mathrm{O}_{3} \text { generated from } \mathrm{O}_{2} \text { at } 100^{\circ} \mathrm{F} \text { and } 1 \mathrm{~atm} . \\
\frac{\text { Reaction }}{3 \mathrm{O}_{2} \rightarrow 2 \mathrm{O}_{3}} \quad \frac{\text { Conversion }}{0.99}\end{array}$ \\
\hline $\begin{array}{l}\text { Aqueous liquids } \\
\text { sorting / } \\
\text { PHOTOMTX } \\
\text { (MIXER) }\end{array}$ & 188 & - & $\begin{array}{l}\text { Combines all streams entering aqueous waste } \\
\text { treatment subsystem. }\end{array}$ \\
\hline $\begin{array}{l}\text { Gross organics } \\
\text { removal / } \\
\text { OILSEP (SEP) }\end{array}$ & 188 & - & $\begin{array}{l}\text { Oil-water separator to remove } \mathrm{C} \text { and } \mathrm{H}_{2} \text { from } \\
\text { liquid stream at an assumed efficiency of } 99 \% \text {. }\end{array}$ \\
\hline $\begin{array}{l}\text { OH generator / } \\
\text { OH-GEN } \\
\text { (RSTOIC) }\end{array}$ & 100 & -160080 & $\begin{array}{l}\text { Generates } \mathrm{OH} \text { radicals from } \mathrm{H}_{2} \mathrm{O}_{2} \text { and } \mathrm{O}_{3} \text {. } \\
\begin{array}{lc}\text { Reaction } & \text { Conversion } \\
\mathrm{H}_{2} \mathrm{O}_{2} \rightarrow 2 \mathrm{OH} & 0.99 \\
\mathrm{O}_{3}+3 \mathrm{H}_{2} \mathrm{O} \rightarrow 6 \mathrm{OH} & 0.99\end{array}\end{array}$ \\
\hline $\begin{array}{l}\text { UV reactor / } \\
\text { PHOTO-UV } \\
\text { (RSTOIC) }\end{array}$ & 100 & -42590 & 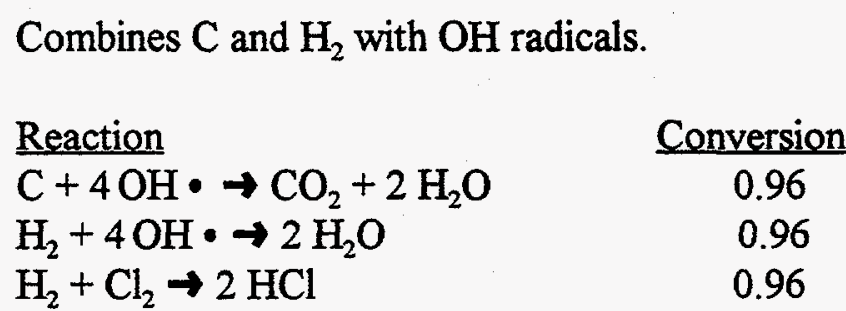 \\
\hline
\end{tabular}




\begin{tabular}{|c|c|c|c|}
\hline $\begin{array}{l}\text { Ozone } \\
\text { decomposition / } \\
\text { OZ-DECOM } \\
\text { (RSTOIC) }\end{array}$ & 100 & -2100 & 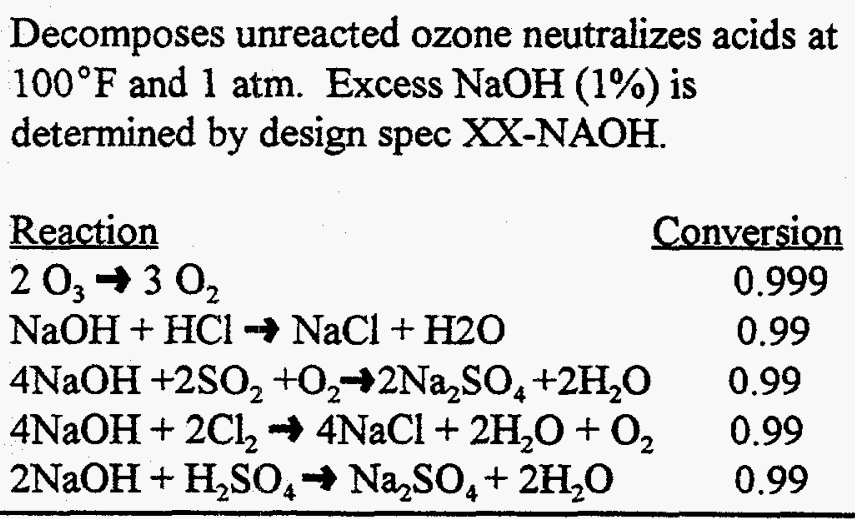 \\
\hline $\begin{array}{l}\text { Gas, water, } \\
\text { sludge separator/ } \\
\text { AQ-SPLIT (SEP) }\end{array}$ & 100 & - & $\begin{array}{l}\text { Separates gas, liquid, and sludge from aqueous } \\
\text { waste treatment. Gases }\left(\mathrm{O}_{2}, \mathrm{H}_{2}, \mathrm{~N}_{2}, \mathrm{~F}_{2}, \mathrm{NO}, \mathrm{CO} \text {, }\right. \\
\mathrm{O}_{3} \text { ) are sent to } \mathrm{APC} \text { subsystem. } \mathrm{Liquids}\left(\mathrm{H}_{2} \mathrm{O},\right. \\
\mathrm{SO}_{2}, \mathrm{H}_{2} \mathrm{SO}_{4}, \mathrm{HCl}, \mathrm{HNO}_{3}, \mathrm{H}_{2} \mathrm{O}_{2}, \mathrm{OH} \text { radicals, } 1 \% \\
\left.\text { of } \mathrm{C}, 1 \% \text { of } \mathrm{SiO}_{2}, 1 \% \text { of } \mathrm{Al}_{2} \mathrm{O}_{3}\right) \text { are available for } \\
\text { recycle in the system. } \mathrm{Solids}(\mathrm{Fe}, \mathrm{As}, \mathrm{Ba}, \mathrm{Cd}, \mathrm{Cr} \text {, } \\
\mathrm{Pb}, \mathrm{Hg}, \mathrm{Se}, \mathrm{Ag}, \mathrm{NaCl}, \mathrm{FeCl} \mathrm{FeCl}_{3}, \mathrm{AgCl}, \mathrm{NaOH} \\
\mathrm{Na}_{2} \mathrm{SO}_{4}, \mathrm{NaNO}_{3}, 99 \% \text { of } \mathrm{C}, 99 \% \text { of } \mathrm{SiO}, 99 \% \text { of } \\
\mathrm{Al}_{2} \mathrm{O}_{3} \text { ) are routed to polymer stabilization } \\
\text { subsystem. }\end{array}$ \\
\hline $\begin{array}{l}\text { Evaporator / } \\
\text { CONCENT8 } \\
\text { (FLASH2) }\end{array}$ & 150 & 111 & $\begin{array}{l}\text { Models evaporation of scrubber liquor from APC } \\
\text { subsystem. Two-phase flash determines vapor- } \\
\text { liquid equilibrium at } 150^{\circ} \mathrm{F} \text { and } 1 \text { atm. Vapor } \\
\text { sent to PHOTOMIX, liquid/solid phase sent to } \\
\text { polymer stabilization subsystem. }\end{array}$ \\
\hline $\begin{array}{l}\text { Soil wash / } \\
\text { AQ-WASH1 } \\
\text { (SEP) }\end{array}$ & 65 & - & $\begin{array}{l}\text { Models the aqueous wash for bulk soils. } \\
\text { Operates at } 65^{\circ} \mathrm{F} \text { and } 1 \mathrm{~atm} \text {. Water is added in a } \\
1: 1 \text { ratio with the incoming soil. } 95 \% \text { of the } \mathrm{H}_{2} \text {, } \\
95 \% \text { of the } \mathrm{Cl}_{2} \text {, and } 95 \% \text { of the } \mathrm{C} \text { are removed by } \\
\text { washing and are routed to the organic destruction } \\
\text { subsystem. }\end{array}$ \\
\hline $\begin{array}{l}\text { Soil dryer / } \\
\text { SOLDRYER } \\
\text { (HEATER) }\end{array}$ & 200 & 54566 & $\begin{array}{l}\text { Dryer removes water entrained in soil during } \\
\text { washing. }\end{array}$ \\
\hline $\begin{array}{l}\text { Soil dryer / } \\
\text { H2OSEP (SEP) }\end{array}$ & 200 & - & $\begin{array}{l}\text { Separates water dried from soil. Water is sent to } \\
\text { the aqueous waste treatment subsystem. Dried } \\
\text { soil is routed to the grout stabilization subsystem. }\end{array}$ \\
\hline
\end{tabular}




\begin{tabular}{|c|c|c|c|}
\hline $\begin{array}{l}\text { Grout } \\
\text { stabilization feed } \\
\text { preparation / } \\
\text { GROUTMIX } \\
\text { (MIXER) }\end{array}$ & 88 & - & Combines all waste feed to grout stabilization. \\
\hline $\begin{array}{l}\text { Micro- } \\
\text { solidification / } \\
\text { GROUTER } \\
\text { (MOXER) }\end{array}$ & 72 & - & $\begin{array}{l}\text { Combines cement and water with incoming waste } \\
\text { feed. Amount of cement is determined by design } \\
\text { spec GROUT. Total grout:waste ratio is } 2: 1 \text { and } \\
\text { dry grout:water ratio is } 0.7: 0.3 \text {. Amount of water } \\
\text { is determined by design spec GROUTH } 20 \\
\text { according to the above ratio. }\end{array}$ \\
\hline $\begin{array}{l}\text { Mercury } \\
\text { amalgamation / } \\
\text { HG-AMALG } \\
\text { (MIXER) }\end{array}$ & 68 & - & $\begin{array}{l}\text { Amalgamation of mercury wastes. Addition of } \\
\text { copper in ratio of } 0.7: 1 \text { (Cu:waste) as determined } \\
\text { by FORTRAN block ADDITIVE. }\end{array}$ \\
\hline $\begin{array}{l}\text { Metal } \\
\text { decontamination / } \\
\text { MET-DCON } \\
\text { (SEP) }\end{array}$ & 64 & - & $\begin{array}{l}\text { Decontamination of metal with abrasive blasting. } \\
\text { Assumes water input in a ratio of } 112 \mathrm{lb} \text { per } 468 \\
\text { lb waste. Assumes all grit recycled (no make-up } \\
\text { grit added to process). Liquid stream }\left(\mathrm{H}_{2} \mathrm{O}, \mathrm{H}_{2} \text {, }\right. \\
\mathrm{C}, \mathrm{Al}_{2} \mathrm{O}_{3} \text {, and } \mathrm{SiO}_{2} \text { ) is sent to aqueous waste } \\
\text { treatment. Clean metal ( } \mathrm{Fe} \text { ) exits the process. }\end{array}$ \\
\hline $\begin{array}{l}\text { Organic } \\
\text { destruction feed } \\
\text { preparation / } \\
\text { MEO-MIXR } \\
\text { (MLXER) }\end{array}$ & 184 & - & $\begin{array}{l}\text { Mixes all feed material to MEO process, including } \\
\text { waste input, reagents, and recycle streams (water } \\
\text { and nitric acid). Make-up silver nitrate is } \\
\text { determined by FORTRAN block AGNO3-IN. } \\
\text { Make-up nitric acid is determined by FORTRAN } \\
\text { block HNO3-IN2. }\end{array}$ \\
\hline $\begin{array}{l}\text { MEO process - } \\
\text { anode / MEO- } \\
\text { ANOD } \\
\text { (RSTOIC) }\end{array}$ & 176 & 64958648 & $\begin{array}{lc}\text { Reacts at } 80^{\circ} \mathrm{C} \text { and } 35 \text { psig. } \\
\text { Reaction } & \\
\mathrm{C}+2 \mathrm{H}_{2} \mathrm{O} \rightarrow \mathrm{CO}_{2}+4 \mathrm{H}^{+}+4 \mathrm{e}^{-} & \text {Conversion } \\
\mathrm{H}_{2} \rightarrow 2 \mathrm{H}^{+} & 0.99 \\
\mathrm{Cl}_{2}+2 \mathrm{H}^{+}+2 \mathrm{e}^{-} \rightarrow 2 \mathrm{HCl} & 1.00 \\
2 \mathrm{~S}+2 \mathrm{H}_{2} \mathrm{O}+3 \mathrm{O}_{2} \rightarrow \mathrm{H}_{2} \mathrm{SO}_{4} & 1.00 \\
2 \mathrm{~N}_{2}+2 \mathrm{H}_{2} \mathrm{O}+5 \mathrm{O}_{2} \rightarrow 4 \mathrm{HNO}_{3} & 1.00 \\
& 1.00\end{array}$ \\
\hline
\end{tabular}




\begin{tabular}{|c|c|c|c|}
\hline $\begin{array}{l}\text { MEO process - } \\
\text { cathode / MEO- } \\
\text { CATH (RSTOIC) }\end{array}$ & 176 & -68479920 & $\begin{array}{l}\text { Reacts at } 80^{\circ} \mathrm{C} \text { and } 35 \text { psig.. } \\
\begin{array}{lc}\text { Reaction } & \text { Conversion } \\
\mathrm{HNO}_{3}+2 \mathrm{H}^{+}+2 \mathrm{e}^{-} \rightarrow \mathrm{H}_{2} \mathrm{O}+\mathrm{HNO}_{2} & 1.00 \\
\mathrm{AgNO}_{3}+\mathrm{HCl} \rightarrow \mathrm{AgCl}+\mathrm{HNO}_{3} & 0.99\end{array}\end{array}$ \\
\hline $\begin{array}{l}\text { Silver } \\
\text { precipitation / } \\
\text { AG-PPT } \\
\text { (RSTOIC) }\end{array}$ & 176 & -496 & $\begin{array}{l}\text { Precipitation of remaining } \mathrm{AgNO}_{3} \text { with addition } \\
\text { of } \mathrm{HCl} \text {. Reacts and } 80^{\circ} \mathrm{C} \text { and } 35 \text { psig at } 100 \% \\
\text { conversion. }\end{array}$ \\
\hline $\begin{array}{l}\text { MEO process / } \\
\text { MEO3 (RSTOIC) }\end{array}$ & 176 & 0 & 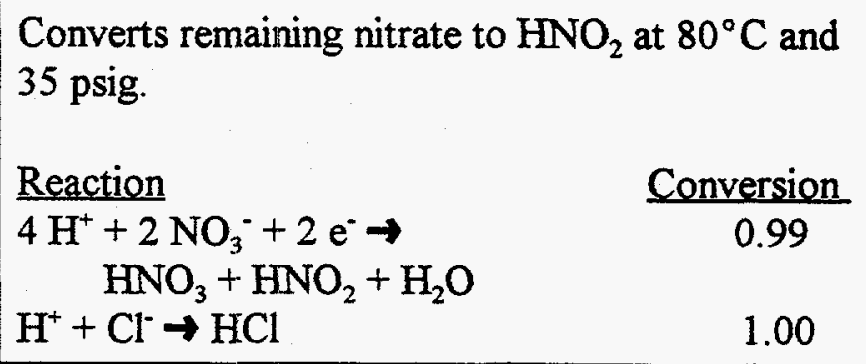 \\
\hline $\begin{array}{l}\text { Centrifuge- } \\
\text { Evaporator / } \\
\text { MEO-SPLT } \\
\text { (SEP) }\end{array}$ & 176 & - & $\begin{array}{l}\text { Separates gases, solids, and recycle streams. } \\
\text { Some } \mathrm{H}_{2} \mathrm{O} \text { and all } \mathrm{HCl} \text { is recycled to } \mathrm{MEO} \\
\text { process and is controlled by design spec } \mathrm{H} 2 \mathrm{O}-\mathrm{IN} \text {. } \\
\text { Remaining } \mathrm{H}_{2} \mathrm{O} \text { is routed aqueous waste } \\
\text { treatment. } \mathrm{Gases} \text { are routed to nitrous acid } \\
\text { conversion and } \mathrm{NO}_{x} \text { removal. } \mathrm{AgCl} \text { and } \mathrm{HNO}_{3} \\
\text { are routed to silver conversion. } \mathrm{F}_{2}, \mathrm{Ba}, \mathrm{Fe}, \mathrm{Cd} \text {, } \\
\mathrm{Cr}, \mathrm{C}, \mathrm{SiO}_{2}, \mathrm{Al}_{2} \mathrm{O}_{3} \text {, and } 10 \mathrm{ppm} \text { of } \mathrm{HNO}_{3} \text { are } \\
\text { routed to neutralization. }\end{array}$ \\
\hline $\begin{array}{l}\text { Nitrous acid } \\
\text { conversion / } \\
\text { HNO2RECV } \\
\text { (RSTOIC) }\end{array}$ & 302 & -1006403 & 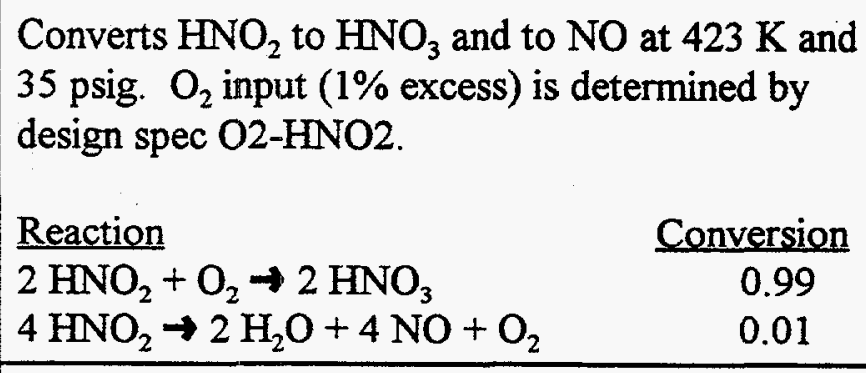 \\
\hline $\begin{array}{l}\text { Nitrous acid } \\
\text { conversion / } \\
\text { NOX-SPLT } \\
\text { (SEP) }\end{array}$ & 302 & - & $\begin{array}{l}\text { Separates } \mathrm{HNO}_{3} \text { for recycle back to MEO } \\
\text { reactor. }\end{array}$ \\
\hline
\end{tabular}




\begin{tabular}{|c|c|c|c|}
\hline $\begin{array}{l}\text { NOx removal / } \\
\text { NOX-CONV } \\
\text { (RSTOIC) }\end{array}$ & 68 & -107747 & $\begin{array}{l}\text { Reacts } \mathrm{NO} \text { at } 68^{\circ} \mathrm{F} \text { and } 1 \text { atm and } 99 \% \\
\text { conversion. Reaction is. Stoichiometric } \mathrm{NH}_{3} \\
\text { input is determined by FORTRAN block X-NH3. } \\
\text { Gases are then routed to APC. } \\
\frac{\text { Reaction }}{6 \mathrm{NO}+4 \mathrm{NH}_{3} \rightarrow 5 \mathrm{~N}_{2}+6 \mathrm{H}_{2} \mathrm{O}} \frac{\text { Conversion }}{0.99}\end{array}$ \\
\hline $\begin{array}{l}\text { Silver conversion- } \\
\text { silver dissolving / } \\
\text { AGRECOVR } \\
\text { (RSTOIC) }\end{array}$ & 100 & 761415 & $\begin{array}{l}\text { Converts } \mathrm{AgCl} \text { to } \mathrm{AgNO}_{3} \text { at } 100^{\circ} \mathrm{F} \text { and } 1 \mathrm{~atm} \text {; } \\
\mathrm{H}_{2} \mathrm{O}_{2} \text { and } \mathrm{NaOH} \text { fed to the block are determined } \\
\text { by the FORTRAN block AGRECOVR. } \\
\begin{array}{l}\text { Reaction } \\
4 \mathrm{AgCl}+4 \mathrm{NaOH}+4 \mathrm{H}_{2} \mathrm{O}_{2} \rightarrow\end{array} \quad \frac{\text { Conversion }}{4 \mathrm{Ag}+4 \mathrm{NaCl}+6 \mathrm{H}_{2} \mathrm{O}+3 \mathrm{O}_{2}} \\
\begin{array}{l}\mathrm{Ag}+\mathrm{HNO}_{3} \rightarrow \mathrm{AgNO}_{3}+\mathrm{H}^{+}+\mathrm{e}^{-} \\
\mathrm{e}^{-}\end{array}\end{array}$ \\
\hline $\begin{array}{l}\text { Centrifuge / } \\
\text { AG-SPLT (SEP) }\end{array}$ & 100 & - & $\begin{array}{l}\text { Separates } \mathrm{AgNO}_{3} \text { and } \mathrm{HNO}_{3} \text { for recycle to MEO } \\
\text { reactor. } \mathrm{Gases}\left(\mathrm{O}_{2}\right) \text { are routed to APC system. } \\
\text { Liquids }\left(\mathrm{H}_{2} \mathrm{O}, \mathrm{Ag}, \mathrm{NaOH}, \mathrm{NaCl}, \mathrm{H}^{+}, \mathrm{e}^{-}, \mathrm{H}_{2} \mathrm{O}_{2} \text {, }\right. \\
\mathrm{AgCl}) \text { are routed to aqueous waste treatment. }\end{array}$ \\
\hline $\begin{array}{l}\text { Neutralization / } \\
\text { NEUTRAL } \\
\text { (RSTOIC) }\end{array}$ & 68 & -406 & 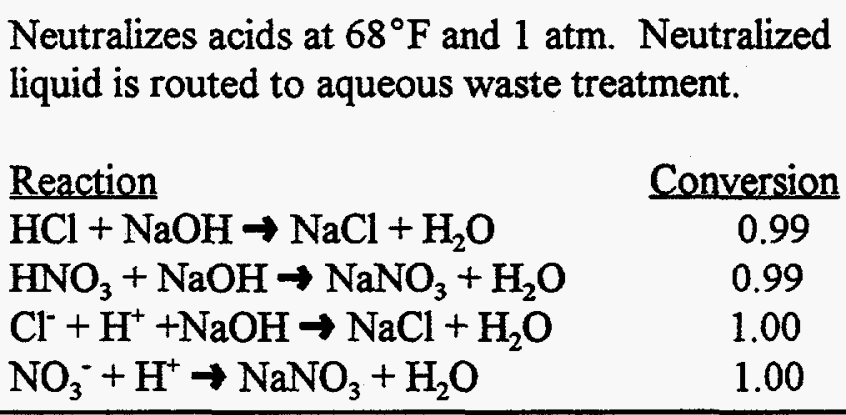 \\
\hline $\begin{array}{l}\text { Lead recovery- } \\
\text { separation / } \\
\text { PB-SPLIT (SEP) }\end{array}$ & 68 & - & $\begin{array}{l}\text { Separates brick or sheet lead (assumed to be } 81 \% \\
\text { of total) for decontamination. Remaining lead } \\
\text { (leaded gloves, blankets, shot) is routed to grout } \\
\text { stabilization. }\end{array}$ \\
\hline $\begin{array}{l}\text { Lead } \\
\text { decontamination / } \\
\text { PB-DECON } \\
\text { (SEP) }\end{array}$ & 63 & - & $\begin{array}{l}\text { Decontaminates lead with water wash, separates } \\
\text { clean lead, which exits the system. Liquid and } \\
\text { sludge are routed to process residue treatment } \\
\text { subsystem. }\end{array}$ \\
\hline $\begin{array}{l}\text { Polymer feed } \\
\text { preparation / } \\
\text { POLY-MIX } \\
\text { (MIXER) }\end{array}$ & 70 & - & Mixes incoming waste for polymer stabilization. \\
\hline
\end{tabular}




\begin{tabular}{|c|c|c|c|}
\hline $\begin{array}{l}\text { Dryer / DRYER } \\
\text { (HEATER) }\end{array}$ & 200 & 91425 & $\begin{array}{l}\text { Removes excess water prior to polymer } \\
\text { stabilization. }\end{array}$ \\
\hline $\begin{array}{l}\text { Dryer / } \\
\text { WATERSEP } \\
\text { (SEP) }\end{array}$ & 200 & - & $\begin{array}{l}\text { Separates water, input to polymer stabilization is } \\
\text { dried to } 5 \% \text { moisture content, as determined by } \\
\text { design spec DRYER. Excess } \mathrm{H}_{2} \mathrm{O}, \mathrm{NO} \text {, and } \mathrm{SO}_{2} \\
\text { are sent to aqueous waste treatment subsystem. }\end{array}$ \\
\hline $\begin{array}{l}\text { Mixing / } \\
\text { POLYSTAB } \\
\text { (MIXER) }\end{array}$ & 96 & - & $\begin{array}{l}\text { Adds polyethylene to encapsulate the waste. } \\
\text { Polymer:waste ratio is } 1: 1 \text {. }\end{array}$ \\
\hline $\begin{array}{l}\text { Process residue } \\
\text { wash / } \\
\text { AQ-WASH2 } \\
\text { (SEP) }\end{array}$ & 67 & - & $\begin{array}{l}\text { Models the aqueous wash for process residue and } \\
\text { inorganic sludges. Make-up water of } 15 \% \text { of } \\
\text { mass of incoming waste is used. The washing } \\
\text { process produces an organic stream }\left(95 \% \text { of } \mathrm{O}_{2} \text {, }\right. \\
95 \% \text { of } \mathrm{H}_{2}, 95 \% \text { of } \mathrm{Cl}_{2}, 95 \% \text { of } \mathrm{C}, 1 \% \text { of } \mathrm{SiO}_{2} \text {, } \\
\left.\text { and } 1 \% \text { of } \mathrm{Al}_{2} \mathrm{O}_{3}\right) \text { that is sent to organic } \\
\text { destruction. The remaining solids } \mathrm{H}_{2} \mathrm{O}, 5 \% \text { of } \\
\mathrm{O}_{2}, 5 \% \text { of } \mathrm{H}_{2}, \mathrm{~N}_{2}, \mathrm{~S}, 5 \% \text { of } \mathrm{Cl}_{2}, \mathrm{Fe}, \mathrm{As}, \mathrm{Ba}, \mathrm{Cd} \text {, } \\
\mathrm{Cr}, \mathrm{Pb}, \mathrm{Hg}, \mathrm{Se}, \mathrm{Ag}, 5 \% \text { of } \mathrm{C}, 99 \% \text { of } \mathrm{SiO}_{2} \text {, and } \\
99 \% \text { of } \mathrm{Al}_{2} \mathrm{O}_{3} \text { ) are routed to the polymer } \\
\text { stabilization subsystem. }\end{array}$ \\
\hline $\begin{array}{l}\text { Soft debris wash } \\
\text { / AQ-WASH3 } \\
\text { (SEP) }\end{array}$ & 64 & - & $\begin{array}{l}\text { Models the aqueous wash for soft debris. Make- } \\
\text { up water of } 15 \% \text { of mass of incoming waste is } \\
\text { used. Two-thirds of the make-up water will be } \\
\text { routed to aqueous waste treatment; one-third of } \\
\text { the water is assumed to be entrained with the } \\
\text { waste after washing. The washing process } \\
\text { produces an aqueous stream ( } 10 \% \text { of } \mathrm{O}_{2}, 10 \% \text { of } \\
\mathrm{H}_{2}, 10 \% \text { of } \mathrm{Cl}_{2}, 10 \% \text { of } \mathrm{F}_{2}, 10 \% \text { of } \mathrm{Hg}, 10 \% \text { of } \mathrm{C} \text {, } \\
1 \% \text { of } \mathrm{SiO}_{2}, \text { and } 1 \% \text { of } \mathrm{Al}_{2} \mathrm{O}_{3} \text {, and two-thirds of } \\
\text { the wash } \mathrm{H}_{2} \mathrm{O} \text { ) that is sent to aqueous waste } \\
\text { treatment. The remaining solids (remainder of } \\
\mathrm{H}_{2} \mathrm{O}, 90 \% \text { of } \mathrm{O}_{2}, 90 \% \text { of } \mathrm{H}_{2}, \mathrm{~N}_{2}, \mathrm{~S}, 90 \% \text { of } \mathrm{Cl}_{2} \text {, } \\
90 \% \text { of } \mathrm{F}_{2}, \mathrm{Fe}, \mathrm{Pb}, 90 \% \text { of } \mathrm{Hg}, \mathrm{Se}_{\mathrm{Ag}}, 90 \% \text { of } \\
\mathrm{C}, 99 \% \text { of } \mathrm{SiO}_{2} \text {, and } 99 \% \text { of } \mathrm{Al}_{2} \mathrm{O}_{3} \text { ) are routed to } \\
\text { the grout stabilization subsystem. }\end{array}$ \\
\hline
\end{tabular}




\begin{tabular}{|c|c|c|c|}
\hline $\begin{array}{l}\text { Open debris wash } \\
\text { / AQ-WASH } 4 \\
\text { (SEP) }\end{array}$ & 66 & - & $\begin{array}{l}\text { Models the aqueous wash for open debris. Make- } \\
\text { up water of } 15 \% \text { of mass of incoming waste is } \\
\text { used. Two-thirds of the make-up water will be } \\
\text { routed to aqueous waste treatment; one-third of } \\
\text { the water is assumed to be entrained with the } \\
\text { waste after washing. The washing process } \\
\text { produces an aqueous stream ( } 98 \% \text { of } \mathrm{O}_{2}, 98 \% \text { of } \\
\mathrm{H}_{2}, 98 \% \text { of } \mathrm{C} \text {, two-thirds of the wash } \mathrm{H}_{2} \mathrm{O}, 1 \% \text { of } \\
\mathrm{SiO}_{2} \text {, and } 1 \% \text { of } \mathrm{Al}_{2} \mathrm{O}_{3} \text { ) that is sent to aqueous } \\
\text { waste treatment. The remaining solids } \\
\text { (remainder of } \mathrm{H}_{2} \mathrm{O}, 2 \% \text { of } \mathrm{O}_{2}, 2 \% \text { of } \mathrm{H}_{2}, \mathrm{~N}_{2}, \mathrm{~S} \text {, } \\
2 \% \text { of } \mathrm{Cl}_{2}, \mathrm{Fe}, \mathrm{As}, \mathrm{Ba}, \mathrm{Cd}, \mathrm{Cr}, \mathrm{Pb}, \mathrm{Hg}_{,} \mathrm{Se}, \mathrm{Ag} \text {, } \\
2 \% \text { of } \mathrm{C}, 99 \% \text { of } \mathrm{SiO}, \text { and } 99 \% \text { of } \mathrm{Al}_{2} \mathrm{O}_{3} \text { ) are } \\
\text { routed to the polymer stabilization subsystem. }\end{array}$ \\
\hline $\begin{array}{l}\text { Complex debris } \\
\text { wash / } \\
\text { AQ-WASH5 } \\
\text { (SEP) }\end{array}$ & 63 & - & $\begin{array}{l}\text { Models the aqueous wash for complex debris. } \\
\text { Make-up water of } 15 \% \text { of mass of incoming } \\
\text { waste is used. Two-thirds of the make-up water } \\
\text { will be routed to aqueous waste treatment; one- } \\
\text { third of the water is assumed to be entrained with } \\
\text { the waste after washing. The washing process } \\
\text { produces an aqueous stream ( } 98 \% \text { of } \mathrm{H}_{2}, 98 \% \text { of } \\
\mathrm{C} \text {, two-thirds of the wash } \mathrm{H}_{2} \mathrm{O}, 1 \% \text { of } \mathrm{SiO}_{2} \text {, and } \\
1 \% \text { of } \mathrm{Al}_{2} \mathrm{O}_{3} \text {.) that is sent to aqueous waste } \\
\text { treatment. The remaining solids (remainder of } \\
\mathrm{H}_{2} \mathrm{O}, 2 \% \text { of } \mathrm{H}_{2}, \mathrm{Fe}, \mathrm{Hg}, 2 \% \text { of C, } 99 \% \text { of } \mathrm{SiO}_{2} \text {, } \\
\text { and } 99 \% \text { of } \mathrm{Al}_{2} \mathrm{O}_{3} \text { ) are routed to the polymer } \\
\text { stabilization subsystem. }\end{array}$ \\
\hline Special waste & 68 & - & $\begin{array}{l}\text { As with the ITTS study, no model was } \\
\text { constructed for special waste. }\end{array}$ \\
\hline
\end{tabular}


System 4 - Acid Digestion Case

\begin{tabular}{|c|c|c|c|}
\hline \multicolumn{4}{|c|}{ ASSUMPTIONS and ENERGY BALANCES for ACID DIGESTION CASE, System 4} \\
\hline \multicolumn{4}{|c|}{ GENERAL COMMENTS: } \\
\hline $\begin{array}{l}\text { Unit Operation / } \\
\text { ASPEN PLUS } \\
\text { Block Name }\end{array}$ & $\begin{array}{l}\text { Outlet } \\
\text { Temp. } \\
\left({ }^{\circ} \mathrm{F}\right) \\
\end{array}$ & $\begin{array}{l}\text { Heat Duty } \\
\text { (MMBtu/hr) }\end{array}$ & Description and Assumptions \\
\hline $\begin{array}{l}\text { APC filter / } \\
\text { FIL TER (SEP) }\end{array}$ & 100 & - & $\begin{array}{l}\text { Filter to separate heavy metals and other solids } \\
\text { from gas stream. Gas streams entering the APC } \\
\text { system usually have all particulates removed } \\
\text { beforehand. }\end{array}$ \\
\hline $\begin{array}{l}\text { Gas phase corona } \\
\text { reactor / GPCR } \\
\text { (RSTOIC) }\end{array}$ & 419 & 40292 &  \\
\hline $\begin{array}{l}\text { Acid gas scrubber } \\
\text { / NEUTRAL3 } \\
\text { (RSTOIC) }\end{array}$ & 100 & -105058 & $\begin{array}{l}\text { Neutralizes } \mathrm{SO}_{2} \text { with } \mathrm{NaOH} \text { at } 1 \text { atm pressure and } \\
100^{\circ} \mathrm{F} \text { to form } \mathrm{Na}_{2} \mathrm{SO}_{4} \text { at a conversion level of } \\
99 \% \text {. Excess } \mathrm{NaOH}(1 \%) \text { is determined by } \\
\text { design spec } \mathrm{NAOH}-3 \text {. } \\
\frac{\text { Reaction }}{2 \mathrm{SO}_{2}+4 \mathrm{NaOH}+\mathrm{O}_{2} \rightarrow 2 \mathrm{Na}_{2} \mathrm{SO}_{4}+2 \mathrm{H}_{2} \mathrm{O}} \frac{\text { Conversion }}{0.99}\end{array}$ \\
\hline $\begin{array}{l}\text { Scrubber liquor } \\
\text { sump / } \\
\text { BLOWDOWN } \\
\text { (SEP) }\end{array}$ & 100 & - & $\begin{array}{l}\text { Separates salts formed in scrubber. } \mathrm{NaOH} \text { and } \\
\mathrm{NaCl} \text { are sent with some water to aqueous waste } \\
\text { treatment subsystem. Water flow rate calculated } \\
\text { for } 20 \% \text { salt solution by design spec SALTSOLN. }\end{array}$ \\
\hline
\end{tabular}




\begin{tabular}{|c|c|c|c|}
\hline $\begin{array}{l}\text { Mist eliminator / } \\
\text { FLASH } \\
\text { (FLASH2) }\end{array}$ & 100 & 0 & $\begin{array}{l}\text { Two-phase flash to separate out additional } \\
\text { moisture. Flash determines vapor-liquid } \\
\text { equilibrium at } 100^{\circ} \mathrm{F} \text { and } 1 \mathrm{~atm} \text {. Moisture sent to } \\
\text { aqueous waste treatment subsystem. }\end{array}$ \\
\hline $\begin{array}{l}\text { Filter / } \\
\text { HETRHEPA } \\
\text { (HEATER) }\end{array}$ & 300 & 64397 & $\begin{array}{l}\text { Heater to raise temperature of gas stream to } \\
300^{\circ} \mathrm{F} \text { to avoid condensate in HEPA filter. }\end{array}$ \\
\hline $\begin{array}{l}\text { Ozone generator / } \\
\text { OZ-GEN } \\
\text { (RSTOIC) }\end{array}$ & 100 & 1165 & $\begin{array}{l}\mathrm{O}_{3} \text { generated from } \mathrm{O}_{2} \text { at } 100^{\circ} \mathrm{F} \text { and } 1 \mathrm{~atm} . \\
\frac{\text { Reaction }}{3 \mathrm{O}_{2} \rightarrow 2 \mathrm{O}_{3}} \quad \frac{\text { Conversion }}{0.99}\end{array}$ \\
\hline $\begin{array}{l}\text { Aqueous liquids } \\
\text { sorting / } \\
\text { PHOTOMIX } \\
\text { (MIXER) }\end{array}$ & 89 & - & $\begin{array}{l}\text { Combines all streams entering aqueous waste } \\
\text { treatment subsystem. }\end{array}$ \\
\hline $\begin{array}{l}\text { Gross organics } \\
\text { removal / } \\
\text { OILSEP (SEP) }\end{array}$ & 89 & - & $\begin{array}{l}\text { Oil-water separator to remove } \mathrm{C} \text { and } \mathrm{H}_{2} \text { from } \\
\text { liquid stream at an assumed efficiency of } 99 \% \text {. }\end{array}$ \\
\hline $\begin{array}{l}\text { OH generator / } \\
\text { OH-GEN } \\
\text { (RSTOIC) }\end{array}$ & 100 & 2671 & 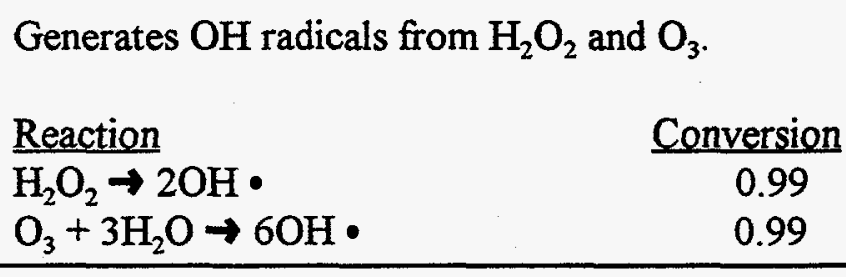 \\
\hline $\begin{array}{l}\text { UV reactor / } \\
\text { PHOTO-UV } \\
\text { (RSTOIC) }\end{array}$ & 100 & -24360 & $\begin{array}{l}\text { Combines } \mathrm{C} \text { and } \mathrm{H}_{2} \text { with } \mathrm{OH} \text { radicals. } \\
\begin{array}{lc}\text { Reaction } & \text { Conversion } \\
\mathrm{C}+4 \mathrm{OH} \cdot \rightarrow \mathrm{CO}_{2}+2 \mathrm{H}_{2} \mathrm{O} & 0.96 \\
\mathrm{H}_{2}+4 \mathrm{OH} \cdot \rightarrow 2 \mathrm{H}_{2} \mathrm{O} & 0.96 \\
\mathrm{H}_{2}+\mathrm{Cl}_{2} \rightarrow 2 \mathrm{HCl} & 0.96 \\
\end{array}\end{array}$ \\
\hline $\begin{array}{l}\text { Ozone } \\
\text { decomposition / } \\
\text { OZ-DECOM } \\
\text { (RSTOIC) }\end{array}$ & 100 & -17033 & 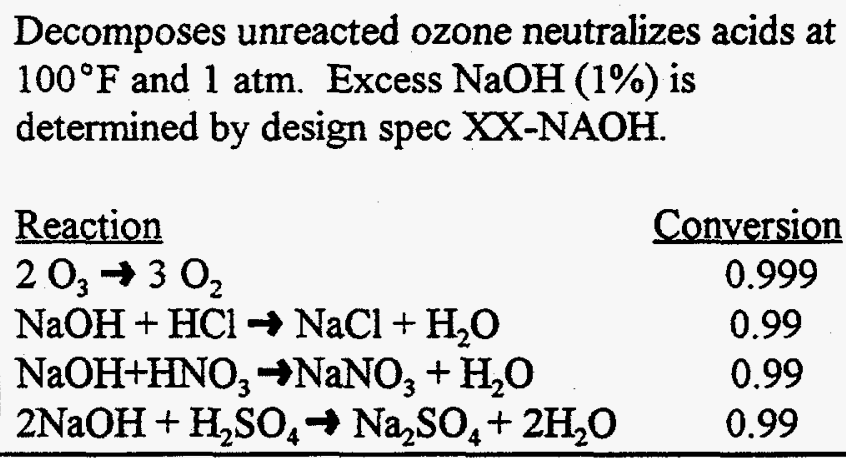 \\
\hline
\end{tabular}




\begin{tabular}{|c|c|c|c|}
\hline $\begin{array}{l}\text { Gas, water, } \\
\text { sludge separator / } \\
\text { AQ-SPLIT (SEP) }\end{array}$ & 100 & - & $\begin{array}{l}\text { Separates gas, liquid, and sludge from aqueous } \\
\text { waste treatment. Gases }\left(\mathrm{O}_{2}, \mathrm{H}_{2}, \mathrm{~N}_{2}, \mathrm{CO}_{2}, \mathrm{O}_{3}\right) \text { are } \\
\text { sent to } \mathrm{APC} \text { subsystem. } \mathrm{Liquids}_{2}\left(\mathrm{H}_{2} \mathrm{O}, \mathrm{SO}_{2},\right. \\
\mathrm{H}_{2} \mathrm{SO}_{4}, \mathrm{HCl}, \mathrm{HNO}_{3}, \mathrm{H}_{2} \mathrm{O}_{2}, \mathrm{NH}_{3}, \mathrm{OH} \text { radicals, } 1 \% \\
\left.\text { of C, } 1 \% \text { of } \mathrm{SiO}_{2}, 1 \% \text { of } \mathrm{Al}_{2} \mathrm{O}_{3}\right) \text { are available for } \\
\text { recycle in the system. Solids }(\mathrm{As}, \mathrm{Ba}, \mathrm{Cd}, \mathrm{Cr}, \mathrm{Pb} \text {, } \\
\mathrm{Hg}, \mathrm{Se}, \mathrm{Ag}, \mathrm{NaCl}, \mathrm{AgCl}, \mathrm{NaOH}, \mathrm{Na}_{2} \mathrm{SO}_{4}, \\
\left.\mathrm{NaNO}_{3}, 99 \% \text { of } \mathrm{C}, 99 \% \text { of } \mathrm{SiO}_{2}, 99 \% \text { of } \mathrm{Al}_{2} \mathrm{O}_{3}\right) \\
\text { are routed to polymer stabilization subsystem. }\end{array}$ \\
\hline $\begin{array}{l}\text { Evaporator } / \\
\text { CONCENT8 } \\
\text { (FLASH2) }\end{array}$ & 150 & 2 & $\begin{array}{l}\text { Models evaporation of scrubber liquor from APC } \\
\text { subsystem. Two-phase flash determines vapor- } \\
\text { liquid equilibrium at } 150^{\circ} \mathrm{F} \text { and } 1 \text { atm. Vapor } \\
\text { sent to PHOTOMIX, liquid/solid phase sent to } \\
\text { polymer stabilization subsystem. }\end{array}$ \\
\hline $\begin{array}{l}\text { Soil wash / AQ- } \\
\text { WASH1 (SEP) }\end{array}$ & 65 & - & $\begin{array}{l}\text { Models the aqueous wash for bulk soils. } \\
\text { Operates at } 65^{\circ} \mathrm{F} \text { and } 1 \text { atm. Water is added in a } \\
1: 1 \text { ratio with the incoming soil. } 95 \% \text { of the } \mathrm{H}_{2} \text {, } \\
95 \% \text { of the } \mathrm{Cl}_{2} \text {, and } 95 \% \text { of the } \mathrm{C} \text { are removed by } \\
\text { washing and are routed to the organic destruction } \\
\text { subsystem. }\end{array}$ \\
\hline $\begin{array}{l}\text { Soil dryer / } \\
\text { SOLDRYER } \\
\text { (HEATER) }\end{array}$ & 200 & 54566 & $\begin{array}{l}\text { Dryer removes water entrained in soil during } \\
\text { washing. }\end{array}$ \\
\hline $\begin{array}{l}\text { Soil dryer / } \\
\text { H2OSEP (SEP) }\end{array}$ & 200 & - & $\begin{array}{l}\text { Separates water dried from soil. Water is sent to } \\
\text { the aqueous waste treatment subsystem. Dried } \\
\text { soil is routed to the grout stabilization subsystem. }\end{array}$ \\
\hline $\begin{array}{l}\text { Grout } \\
\text { stabilization feed } \\
\text { preparation / } \\
\text { GROUTMIX } \\
\text { (MIXER) }\end{array}$ & 68 & - & $\begin{array}{l}\text { Combines all waste feed to grout stabilization, } \\
\text { including complex debris. }\end{array}$ \\
\hline $\begin{array}{l}\text { Micro- } \\
\text { solidification / } \\
\text { GROUTER } \\
\text { (MIXER) }\end{array}$ & 56 & - & $\begin{array}{l}\text { Combines cement and water with incoming waste } \\
\text { feed. Amount of cement is determined by design } \\
\text { spec GROUT. Total grout:waste ratio is } 2: 1 \text { and } \\
\text { dry grout:water ratio is } 0.7: 0.3 \text {. Amount of water } \\
\text { is determined by design spec GROUTH } 20 \\
\text { according to the above ratio. }\end{array}$ \\
\hline
\end{tabular}




\begin{tabular}{|c|c|c|c|}
\hline $\begin{array}{l}\text { Mercury } \\
\text { amalgamation / } \\
\text { HG-AMALG } \\
\text { (MIXER) }\end{array}$ & 68 & - & $\begin{array}{l}\text { Amalgamation of mercury wastes. Addition of } \\
\text { copper in ratio of } 0.7: 1 \text { (Cu:waste) as determined } \\
\text { by FORTRAN block ADDITIVE. }\end{array}$ \\
\hline $\begin{array}{l}\text { Metal } \\
\text { decontamination / } \\
\text { MET-DCON } \\
\text { (SEP) }\end{array}$ & 64 & - & $\begin{array}{l}\text { Decontamination of metal with abrasive blasting. } \\
\text { Assumes water input in a ratio of } 112 \mathrm{lb} \text { per } 468 \\
\mathrm{lb} \text { waste. Assumes all grit recycled (no make-up } \\
\text { grit added to process). Liquid stream }\left(\mathrm{H}_{2} \mathrm{O}, \mathrm{H}_{2} \text {, }\right. \\
\left.\mathrm{C}, \mathrm{Al}_{2} \mathrm{O}_{3} \text {, and } \mathrm{SiO}_{2}\right) \text { is sent to aqueous waste } \\
\text { treatment. Clean metal }(\mathrm{Fe} \text { ) exits the process. }\end{array}$ \\
\hline $\begin{array}{l}\text { Digestion reactor } \\
\text { / ACD-DGl } \\
\text { (RSTOIC) }\end{array}$ & 392 & -2118832 & 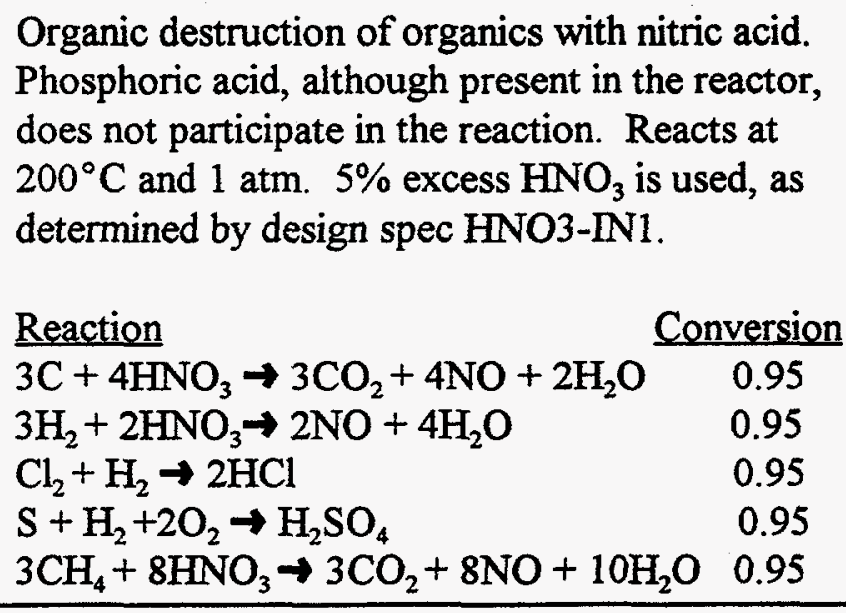 \\
\hline $\begin{array}{l}\text { Acid recovery/ } \\
\text { HNO2RECV } \\
\text { (RSTOIC) }\end{array}$ & 302 & -1093339 & $\begin{array}{l}\text { Recovers nitric acid from NO produced in } \\
\text { digestion reactor. Reaction proceeds at } 423 \mathrm{~K} \\
\text { and } 35 \text { psig. Amount of } \mathrm{O}_{2}(1 \% \text { excess }) \\
\text { determined by design spec OXYGEN1. } \\
\frac{\text { Reaction }}{4 \mathrm{NO}+3 \mathrm{O}_{2}}+2 \mathrm{H}_{2} \mathrm{O} \rightarrow 4 \mathrm{HNO}_{3} \quad \frac{\text { Conversion }}{0.99}\end{array}$ \\
\hline $\begin{array}{l}\text { Acid recovery / } \\
\text { HNO3-SEP } \\
\text { (SEP) }\end{array}$ & 302 & - & $\begin{array}{l}\text { Separates } \mathrm{HNO}_{3} \text { for recycle to digestion reactor. } \\
\text { Assumes a } 99 \% \text { separation of } \mathrm{HNO}_{3} \text {. }\end{array}$ \\
\hline
\end{tabular}




\begin{tabular}{|c|c|c|c|}
\hline $\begin{array}{l}\text { Acid gas scrubber } \\
\text { / NEUTRAL1 } \\
\text { (RSTOIC) }\end{array}$ & 100 & -419798 & 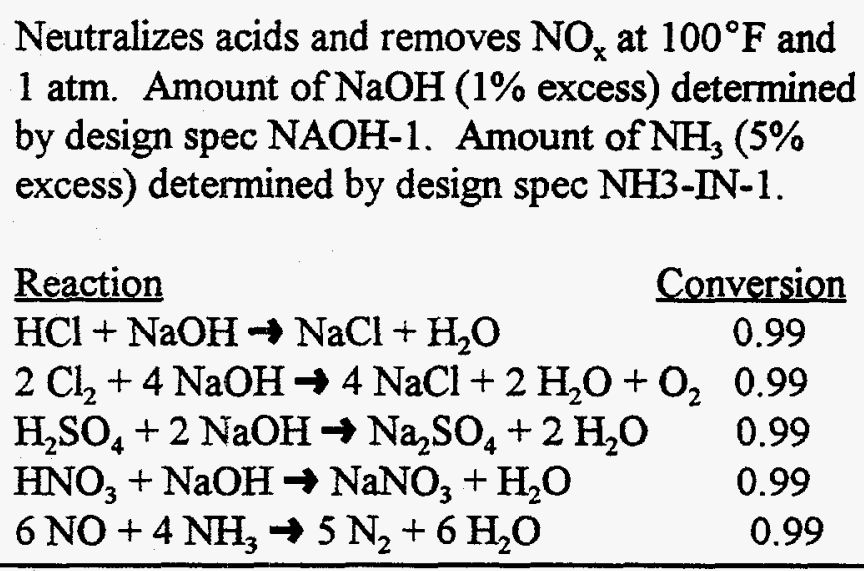 \\
\hline $\begin{array}{l}\text { Acid gas scrubber } \\
\text { / DG-SPLT1 } \\
\text { (SEP) }\end{array}$ & 100 & - & $\begin{array}{l}\text { Separates products of reaction from acid } \\
\text { digestion process. Gases }\left(\mathrm{N}_{2}, \mathrm{O}_{2}, \mathrm{Cl}_{2}, \mathrm{NO}, \mathrm{CO}_{2} \text {, }\right. \\
\left.\text { and } \mathrm{CH}_{4}\right) \text { are routed to the APC subsystem. } \\
\mathrm{Liquids}\left(\mathrm{H}_{2} \mathrm{O}, \mathrm{NaOH}, \mathrm{NaCl}, \mathrm{HCl}, \mathrm{H}_{2} \mathrm{SO}_{4},\right. \\
\left.\mathrm{Na}_{2} \mathrm{SO}_{4}, \mathrm{HNO}_{3}, \mathrm{NaNO}_{3}, \mathrm{NH}_{3}\right) \text { are sent to the } \\
\text { aqueous waste treatment subsystem. Solids }(\mathrm{S}, \\
\left.\mathrm{Fe}, \mathrm{Ba}, \mathrm{Cd}, \mathrm{Cr}, \mathrm{C}, \mathrm{SiO}_{2}, \mathrm{Al}_{2} \mathrm{O}_{3}\right) \text { are sent to } \\
\text { phosphate bonded ceramic stabilization. }\end{array}$ \\
\hline $\begin{array}{l}\text { Lead recovery- } \\
\text { separation / } \\
\text { PB-SPLIT (SEP) }\end{array}$ & 68 & - & $\begin{array}{l}\text { Separates brick or sheet lead (assumed to be } 81 \% \\
\text { of total) for decontamination. Remaining lead } \\
\text { (leaded gloves, blankets, shot) is routed to grout } \\
\text { stabilization. }\end{array}$ \\
\hline $\begin{array}{l}\text { Lead } \\
\text { decontamination / } \\
\text { PB-DECON } \\
\text { (SEP) }\end{array}$ & 63 & - & $\begin{array}{l}\text { Decontaminates lead with water wash, separates } \\
\text { clean lead, which exits the system. Liquid and } \\
\text { sludge are routed to process residue treatment } \\
\text { subsystem. }\end{array}$ \\
\hline $\begin{array}{l}\text { Phosphate } \\
\text { bonded ceramic } \\
\text { feed preparation / } \\
\text { PHOS-MIX } \\
\text { (MIXER) }\end{array}$ & 160 & - & Mixes incoming waste for polymer stabilization. \\
\hline $\begin{array}{l}\text { Dryer / DRYER } \\
\text { (HEATER) }\end{array}$ & 200 & 104038 & $\begin{array}{l}\text { Removes excess water prior to polymer } \\
\text { stabilization. }\end{array}$ \\
\hline $\begin{array}{l}\text { Dryer / } \\
\text { WATERSEP } \\
\text { (SEP) }\end{array}$ & 200 & - & $\begin{array}{l}\text { Separates water, input to polymer stabilization is } \\
\text { dried to } 5 \% \text { moisture content, as determined by } \\
\text { design spec DRYER. Excess } \mathrm{H}_{2} \mathrm{O}, \mathrm{NO} \text {, and } \mathrm{SO}_{2} \\
\text { are sent to aqueous waste treatment subsystem. }\end{array}$ \\
\hline
\end{tabular}




\begin{tabular}{|c|c|c|c|}
\hline $\begin{array}{l}\text { Mixing / } \\
\text { POLYSTAB } \\
\text { (MIXER) }\end{array}$ & 154 & - & $\begin{array}{l}\text { Adds polyethylene to encapsulate the waste. } \\
\text { Polymer:waste ratio is } 1: 1 \text {. }\end{array}$ \\
\hline $\begin{array}{l}\text { Process residue } \\
\text { dryer / } \\
\text { VACTRAX1 } \\
\text { (RGIBBS) }\end{array}$ & 550 & 515629 & $\begin{array}{l}\text { Models the vacuum desorber for process residue } \\
\text { and inorganic sludges. Operates at } 550^{\circ} \mathrm{F} \text { and } 1 \\
\text { psig. Equilibrium is assumed for species entered } \\
\text { in the RGIBBS block: } \mathrm{H}_{2} \mathrm{SO}_{4}, \mathrm{HCl} \text {, and } \mathrm{CH}_{4} \text { are } \\
\text { assumed to form from feed components. } \mathrm{O}_{2} \text { is } \\
\text { excluded from reaction. A nitrogen sweep gas is } \\
\text { used at a rate of } 1.09 \mathrm{ft}^{3} \text { per lb waste }(0.0793 \mathrm{lb} \\
\mathrm{N}_{2} / \mathrm{b} \text { waste) as determined by FORTRAN block } \\
\mathrm{N} 2 \mathrm{SWEEP} \text {. }\end{array}$ \\
\hline $\begin{array}{l}\text { Mercury leaching } \\
\text { / VACSEP-1 } \\
\text { (SEP) }\end{array}$ & 97 & - & $\begin{array}{l}\text { Mercury leaching of process residue and inorganic } \\
\text { sludges by addition of leach water ( } 30 \% \text { of mass } \\
\text { of incoming waste). Also includes separation of } \\
\text { gases, liquids, organics, Hg-contaminated liquids, } \\
\text { and solids. } \\
\text { Gases }\left(\mathrm{N}_{2}\right) \text { are sent to } \mathrm{APC} \text { subsystem. Solids } \\
\left(\mathrm{H}_{2} \mathrm{O} \text { in leach water, } 50 \% \text { of } \mathrm{H}_{2}, \mathrm{O}_{2}, \mathrm{~S}, 50 \% \text { of C, }\right. \\
\mathrm{SiO}_{2}, \mathrm{Al}_{2} \mathrm{O}_{3}, \mathrm{Ba}, \mathrm{As}, \mathrm{Pb}, \mathrm{Fe}, \mathrm{Cd}, \mathrm{Cr}, 5 \% \text { of } \mathrm{Hg} \text {, } \\
\mathrm{Se}, \mathrm{Ag} \text { ) are routed to grout stabilization } \\
\left.\text { subsystem. Liquids (balance of } \mathrm{H}_{2} \mathrm{O}, \mathrm{H}_{2} \mathrm{SO}_{4}\right) \text { are } \\
\text { sent to aqueous waste treatment. Organics }(50 \% \\
\text { of } \mathrm{H}_{2}, 50 \% \text { of } \mathrm{C} \text { ) are routed to the organic } \\
\text { destruction subsystem. Mercury separated }(95 \%) \\
\text { is routed to mercury amalgamation. }\end{array}$ \\
\hline $\begin{array}{l}\text { Soft debris } \\
\text { digestion reactor / } \\
\text { ACID-DG2 } \\
\text { (RSTOIC) }\end{array}$ & 392 & -29288296 & 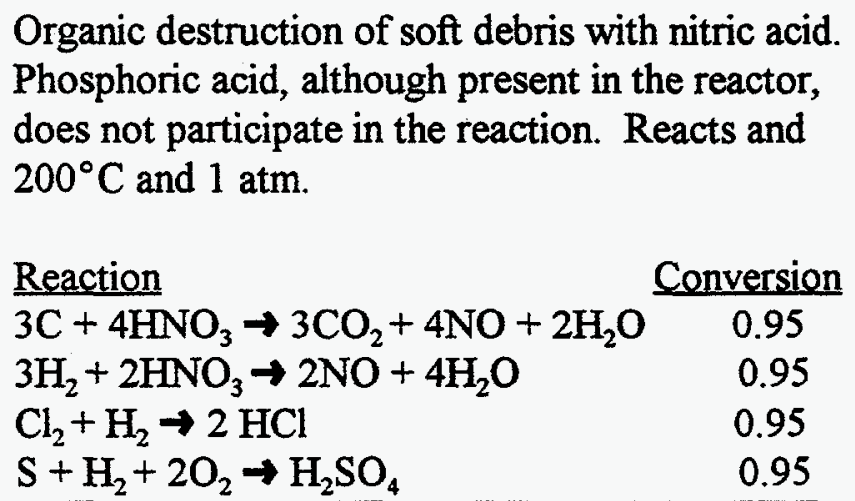 \\
\hline
\end{tabular}




\begin{tabular}{|c|c|c|c|}
\hline $\begin{array}{l}\text { Acid recovery / } \\
\text { HNO2REC2 } \\
\text { (RSTOIC) }\end{array}$ & 302 & -1518081 & $\begin{array}{l}\text { Recovers nitric acid from NO produced in } \\
\text { digestion reactor. Reaction proceeds at } 423 \mathrm{~K} \\
\text { and } 35 \text { psig. Amount of } \mathrm{O}_{2}(0 \% \text { excess) is } \\
\text { determined by design spec } \mathrm{OXYGEN} 2 \text {. Oxygen } \\
\text { in soft debris not available for conversion to } \\
\mathrm{HNO}_{3} \text {. } \\
\frac{\text { Reaction }}{4 \mathrm{NO}+3 \mathrm{O}_{2}+2 \mathrm{H}_{2} \mathrm{O} \rightarrow 4 \mathrm{HNO}_{3} \quad \frac{\text { Conversion }}{0.99}}\end{array}$ \\
\hline $\begin{array}{l}\text { Acid recovery / } \\
\text { HNO3SEP2 } \\
\text { (SEP) }\end{array}$ & 302 & - & $\begin{array}{l}\text { Separates } \mathrm{HNO}_{3} \text { for recycle to digestion reactor. } \\
\text { Assumes a } 99 \% \text { separation of } \mathrm{HNO}_{3} \text {. }\end{array}$ \\
\hline $\begin{array}{l}\text { Acid gas scrubber } \\
\text { / NEUTRAL2 } \\
\text { (RSTOIC) }\end{array}$ & 100 & -470362 & 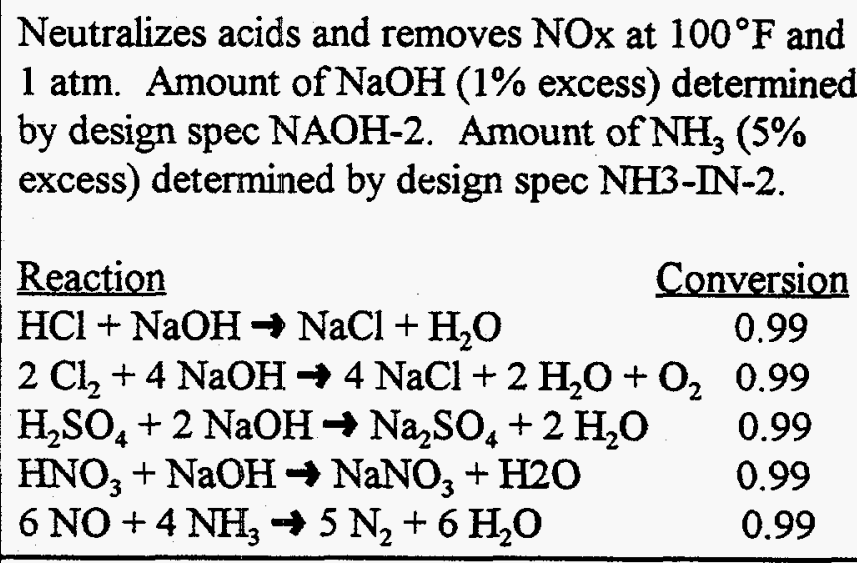 \\
\hline $\begin{array}{l}\text { Acid gas scrubber } \\
\text { / DG-SPLT2 } \\
\text { (SEP) }\end{array}$ & 100 & - & $\begin{array}{l}\text { Separates products of reaction from acid } \\
\text { digestion process. Gases }\left(\mathrm{H}_{2}, \mathrm{~N}_{2}, \mathrm{Cl}_{2}, \mathrm{~F}_{2}, \mathrm{NO} \text {, }\right. \\
\left.\mathrm{CO}_{2}\right) \text { are routed to the } \mathrm{APC} \text { subsystem. Liquids } \\
\left(\mathrm{H}_{2} \mathrm{O}, 5 \% \text { of } \mathrm{Hg}, \mathrm{NaOH}, \mathrm{NaCl}, \mathrm{HCl}, \mathrm{H}_{2} \mathrm{SO}_{4}\right. \\
\left.\mathrm{Na}_{2} \mathrm{SO}_{4}, \mathrm{HNO}_{3}, \mathrm{NaNO}_{3}, \mathrm{NH}_{3}\right) \text { are sent to the } \\
\text { aqueous waste treatment subsystem. Solids }\left(\mathrm{O}_{2} \text {, }\right. \\
\left.\mathrm{S}, \mathrm{Fe}, 95 \% \text { of } \mathrm{Hg}, \mathrm{C}, \mathrm{SiO}_{2}, \mathrm{Al}_{2} \mathrm{O}_{3}\right) \text { are sent to } \\
\text { phosphate bonded ceramic stabilization. }\end{array}$ \\
\hline
\end{tabular}




\begin{tabular}{|l|l|l|l|}
\hline $\begin{array}{l}\text { Open debris wash } \\
\text { / HP-WASH } \\
\text { (SEP) }\end{array}$ & 66 & $\begin{array}{l}\text { Models the aqueous wash for open debris. Make- } \\
\text { up water of } 15 \% \text { of mass of incoming waste is } \\
\text { used. Two-thirds of the make-up water will be } \\
\text { routed to aqueous waste treatment; one-third of } \\
\text { the water is assumed to be entrained with the } \\
\text { waste after washing. The washing process } \\
\text { produces an aqueous stream }\left(98 \% \text { of } \mathrm{O}_{2}, 98 \% \text { of }\right. \\
\mathrm{H}_{2}, 98 \% \text { of } \mathrm{C}, \mathrm{two}-\mathrm{thirds} \text { of the wash water, } 1 \% \\
\text { of } \mathrm{SiO}_{2}, \text { and } 1 \% \text { of } \mathrm{Al}_{2} \mathrm{O}_{3} \text { ) that is sent to aqueous } \\
\text { waste treatment. The remaining solids } \\
\text { (remainder of } \mathrm{H}_{2} \mathrm{O}, 2 \% \text { of } \mathrm{O}_{2}, 2 \% \text { of } \mathrm{H}_{2}, \mathrm{~N}_{2}, \mathrm{~S}, \\
2 \% \text { of } \mathrm{Cl}_{2}, \mathrm{Fe}, \mathrm{As}, \mathrm{Ba}, \mathrm{Cd}, \mathrm{Cr}, \mathrm{Pb}, \mathrm{Hg}, \mathrm{Se}, \mathrm{Ag}, \\
\left.2 \% \text { of } \mathrm{C}, 99 \% \text { of } \mathrm{SiO}, \text { and } 99 \% \text { of } \mathrm{Al} \mathrm{O}_{3}\right) \text { are } \\
\text { routed to the grout stabilization subsystem. }\end{array}$ \\
\hline Special waste & 68 & $\begin{array}{l}\text { As with the ITTS study, no model was } \\
\text { constructed for special waste. }\end{array}$ \\
\hline
\end{tabular}




\section{System 5 - Catalytic Wet Oxidation Case}

\begin{tabular}{|c|c|c|c|}
\hline \multicolumn{4}{|c|}{$\begin{array}{l}\text { ASSUMPTIONS and ENERGY BALANCES for CATALYTIC WET OXIDATION CASE, } \\
\text { System } 5\end{array}$} \\
\hline \multicolumn{4}{|c|}{ GENERAL COMMENTS: } \\
\hline $\begin{array}{l}\text { Unit Operation/ } \\
\text { ASPEN PLUS } \\
\text { Block Name }\end{array}$ & $\begin{array}{l}\text { Outlet } \\
\text { Temp. } \\
\left({ }^{\circ} \mathrm{F}\right) \\
\end{array}$ & $\begin{array}{l}\text { Heat Duty } \\
\text { (MMBtu/hr) }\end{array}$ & Description and Assumptions \\
\hline $\begin{array}{l}\text { APC filter / } \\
\text { FILTER (SEP) }\end{array}$ & 288 & - & $\begin{array}{l}\text { Filter to separate heavy metals and other solids } \\
\text { from gas stream. Gas streams entering the APC } \\
\text { system usually have all particulates removed } \\
\text { beforehand. }\end{array}$ \\
\hline $\begin{array}{l}\text { Gas phase corona } \\
\text { reactor / } \\
\text { GPCR (RSTOIC) }\end{array}$ & 405 & 39148 & $\begin{array}{l}\text { Combines } \mathrm{C}, \mathrm{CO}, \mathrm{H}_{2} \text {, and } \mathrm{CH}_{4} \text { with } \mathrm{O}_{2} \text { at } 1 \text { atm } \\
\text { pressure to form } \mathrm{H}_{2} \mathrm{O} \text { and } \mathrm{CO}_{2} \text {. } \mathrm{Cl}_{2} \text { is combined } \\
\text { with } \mathrm{H}_{2} \text { to form } \mathrm{HCl} \text {. Power input is determined } \\
\text { by design spec } \mathrm{GPCRPOWR} \text {, which calculates } \\
56.49 \mathrm{~W} \text { per } \mathrm{SCFM} \text { of gases entering the process. } \\
\text { The need for excess } \mathrm{O}_{2}(100 \%) \text { is determined by } \\
\text { design spec AIR4. } \\
\begin{array}{l}\text { Reaction } \\
2 \mathrm{H}_{2}+\mathrm{O}_{2} \rightarrow 2 \mathrm{H}_{2} \mathrm{O}\end{array} \\
\mathrm{C}+\mathrm{O}_{2} \rightarrow \mathrm{CO}_{2} \\
2 \mathrm{CO}^{2} \mathrm{O}_{2} \rightarrow 2 \mathrm{CO}_{2} \\
\mathrm{CH}_{4}+2 \mathrm{O}_{2} \rightarrow \mathrm{CO}_{2}+2 \mathrm{H}_{2} \mathrm{O}\end{array}$ \\
\hline $\begin{array}{l}\text { Acid gas scrubber } \\
\text { / NEUTRAL3 } \\
\text { (RSTOIC) }\end{array}$ & 100 & -98578 & 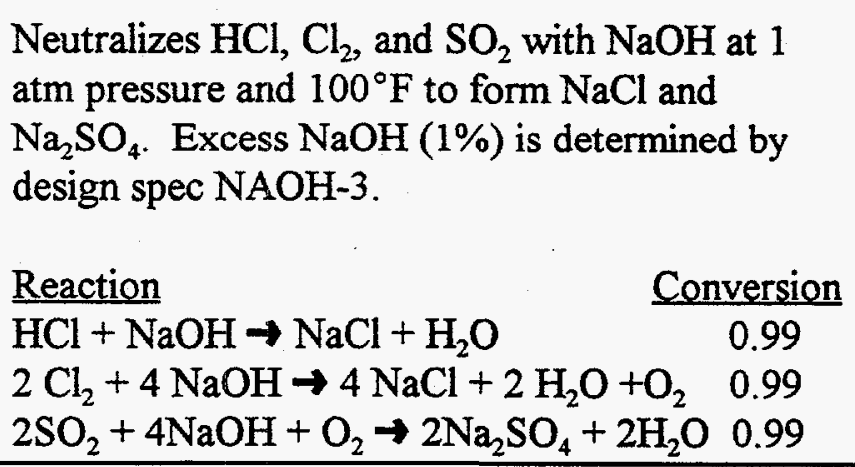 \\
\hline
\end{tabular}




\begin{tabular}{|c|c|c|c|}
\hline $\begin{array}{l}\text { Scrubber liquor } \\
\text { sump / } \\
\text { BLOWDOWN } \\
\text { (SEP) }\end{array}$ & 100 & - & $\begin{array}{l}\text { Separates salts formed in scrubber. } \mathrm{NaCl}, \mathrm{NaOH} \text {, } \\
\text { and } \mathrm{Na}_{2} \mathrm{SO}_{4} \text { are sent with some water to aqueous } \\
\text { waste treatment subsystem. Water flow rate } \\
\text { calculated for } 20 \% \text { salt solution by design spec } \\
\text { SALTSOLN. }\end{array}$ \\
\hline $\begin{array}{l}\text { Mist eliminator / } \\
\text { FLASH } \\
\text { (FLASH2) }\end{array}$ & 100 & 0 & $\begin{array}{l}\text { Two-phase flash to separate out additional } \\
\text { moisture. Flash determines vapor-liquid } \\
\text { equilibrium at } 100^{\circ} \mathrm{F} \text { and } 1 \mathrm{~atm} \text {. Moisture sent to } \\
\text { aqueous waste treatment subsystem. }\end{array}$ \\
\hline $\begin{array}{l}\text { Filter / } \\
\text { HETRHEPA } \\
\text { (HEATER) }\end{array}$ & 300 & 63421 & $\begin{array}{l}\text { Heater to raise temperature of gas stream to } \\
300^{\circ} \mathrm{F} \text { to avoid condensate in HEPA filter. }\end{array}$ \\
\hline $\begin{array}{l}\text { Ozone generator / } \\
\text { OZ-GEN } \\
\text { (RSTOIC) }\end{array}$ & 100 & 1196 & $\begin{array}{l}\mathrm{O}_{3} \text { generated from } \mathrm{O}_{2} \text { at } 100^{\circ} \mathrm{F} \text { and } 1 \mathrm{~atm} . \\
\frac{\text { Reaction }}{3 \mathrm{O}_{2} \rightarrow 2 \mathrm{O}_{3}} \quad \frac{\text { Conversion }}{0.99}\end{array}$ \\
\hline $\begin{array}{l}\text { Aqueous liquids } \\
\text { sorting / } \\
\text { PHOTOMIX } \\
\text { (MIXER) }\end{array}$ & 89 & - & $\begin{array}{l}\text { Combines all streams entering aqueous waste } \\
\text { treatment subsystem. }\end{array}$ \\
\hline $\begin{array}{l}\text { Gross organics } \\
\text { removal / } \\
\text { OILSEP (SEP) }\end{array}$ & 89 & - & $\begin{array}{l}\text { Oil-water separator to remove } \mathrm{C} \text { and } \mathrm{H}_{2} \text { from } \\
\text { liquid stream at an assumed efficiency of } 99 \% \text {. }\end{array}$ \\
\hline $\begin{array}{l}\text { OH generator / } \\
\text { OH-GEN } \\
\text { (RSTOIC) }\end{array}$ & 100 & 1097 & 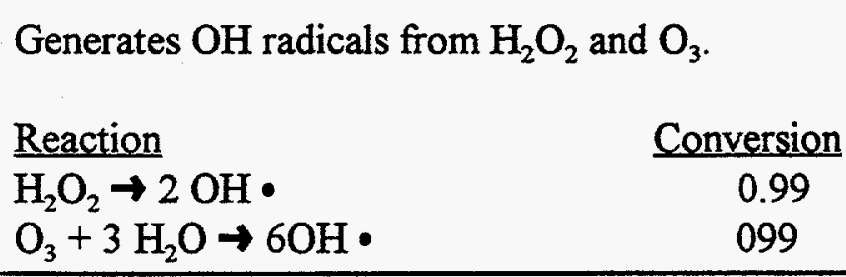 \\
\hline $\begin{array}{l}\text { UV reactor / } \\
\text { PHOTO-UV } \\
\text { (RSTOIC) }\end{array}$ & 100 & -22756 & 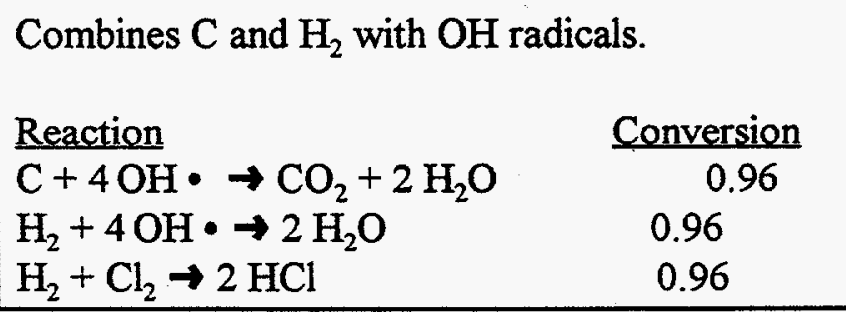 \\
\hline
\end{tabular}




\begin{tabular}{|c|c|c|c|}
\hline $\begin{array}{l}\text { Ozone } \\
\text { decomposition / } \\
\text { OZ-DECOM } \\
\text { (RSTOIC) }\end{array}$ & 100 & -22116 & 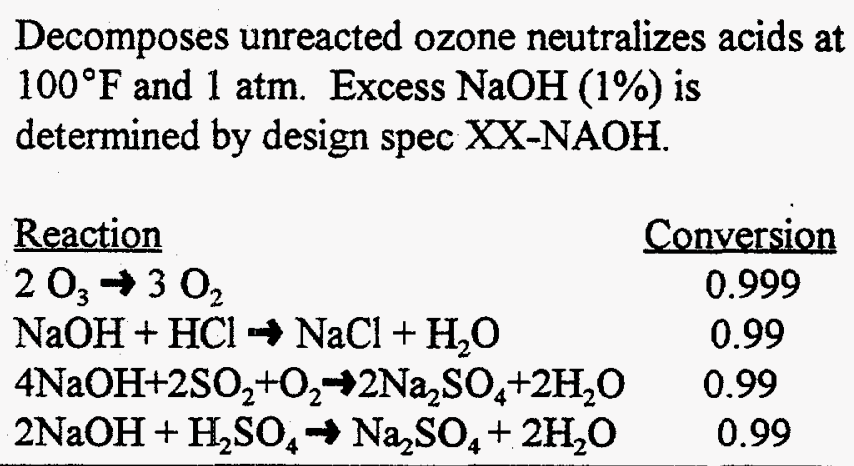 \\
\hline $\begin{array}{l}\text { Gas, water, } \\
\text { sludge separator / } \\
\text { AQ-SPLIT (SEP) }\end{array}$ & 100 & - & $\begin{array}{l}\text { Separates gas, liquid, and sludge from aqueous } \\
\text { waste treatment. Gases }\left(\mathrm{O}_{2}, \mathrm{H}_{2}, \mathrm{~N}_{2}, \mathrm{NO}, \mathrm{CO}_{2} \text {, }\right. \\
\mathrm{O}_{3} \text { ) are sent to } \mathrm{APC} \text { subsystem. Liquids }\left(\mathrm{H}_{2} \mathrm{O} \text {, }\right. \\
\mathrm{SO}_{2}, \mathrm{H}_{2} \mathrm{SO}_{4}, \mathrm{HCl}, \mathrm{H}_{2} \mathrm{O}_{2}, \mathrm{OH} \text { radicals, } 1 \% \text { of } \mathrm{C}, \\
\left.1 \% \text { of } \mathrm{SiO}_{2}, 1 \% \text { of } \mathrm{Al}_{2} \mathrm{O}_{3}\right) \text { are available for recycle } \\
\text { in the system. Solids }(\mathrm{As}, \mathrm{Ba}, \mathrm{Cd}, \mathrm{Cr}, \mathrm{Pb}, \mathrm{Se}, \mathrm{Ag} \text {, } \\
\mathrm{NaCl}, \mathrm{FeCl}_{3}, \mathrm{AgCl}, \mathrm{NaOH}, \mathrm{Na}_{2} \mathrm{SO}_{4}, 99 \% \text { of } \mathrm{C}, \\
\left.99 \% \text { of } \mathrm{SiO}_{2}, 99 \% \text { of } \mathrm{Al}_{2} \mathrm{O}_{3}\right) \text { are routed to } \\
\text { polymer stabilization subsystem. }\end{array}$ \\
\hline $\begin{array}{l}\text { Evaporator / } \\
\text { CONCENT8 } \\
\text { (FLASH2) }\end{array}$ & 150 & 112 & $\begin{array}{l}\text { Models evaporation of scrubber liquor from APC } \\
\text { subsystem. Two-phase flash determines vapor- } \\
\text { liquid equilibrium at } 150^{\circ} \mathrm{F} \text { and } 1 \text { atm. Vapor } \\
\text { sent to PHOTOMIX, liquid/solid phase sent to } \\
\text { polymer stabilization subsystem. }\end{array}$ \\
\hline $\begin{array}{l}\text { Soil wash / AQ- } \\
\text { WASH1 (SEP) }\end{array}$ & 65 & - & $\begin{array}{l}\text { Models the aqueous wash for bulk soils. } \\
\text { Operates at } 65^{\circ} \mathrm{F} \text { and } 1 \text { atm. Water is added in a } \\
1: 1 \text { ratio with the incoming soil. } 95 \% \text { of the } \mathrm{H}_{2} \text {, } \\
95 \% \text { of the } \mathrm{Cl}_{2} \text {, and } 95 \% \text { of the } \mathrm{C} \text { are removed by } \\
\text { washing and are routed to the organic destruction } \\
\text { subsystem. }\end{array}$ \\
\hline $\begin{array}{l}\text { Soil dryer / } \\
\text { SOLDRYER } \\
\text { (HEATER) }\end{array}$ & 200 & 54566 & $\begin{array}{l}\text { Dryer removes water entrained in soil during } \\
\text { washing. }\end{array}$ \\
\hline $\begin{array}{l}\text { Soil dryer / } \\
\text { H2OSEP (SEP) }\end{array}$ & 200 & - & $\begin{array}{l}\text { Separates water dried from soil. Water is sent to } \\
\text { the aqueous waste treatment subsystem. Dried } \\
\text { soil is routed to the grout stabilization subsystem. }\end{array}$ \\
\hline
\end{tabular}




\begin{tabular}{|c|c|c|c|}
\hline $\begin{array}{l}\text { Grout } \\
\text { stabilization feed } \\
\text { preparation / } \\
\text { GROUTMTX } \\
\text { (MIXER) }\end{array}$ & 152 & - & $\begin{array}{l}\text { Combines all waste feed to grout stabilization, } \\
\text { including complex debris. }\end{array}$ \\
\hline $\begin{array}{l}\text { Micro- } \\
\text { solidification / } \\
\text { GROUTER } \\
\text { (MIXER) }\end{array}$ & 83 & - & $\begin{array}{l}\text { Combines cement and water with incoming waste } \\
\text { feed. Amount of cement is determined by design } \\
\text { spec GROUT. Total grout:waste ratio is } 2: 1 \text { and } \\
\text { dry grout:water ratio is } 0.7: 0.3 \text {. Amount of water } \\
\text { is determined by design spec GROUTH } 20 \\
\text { according to the above ratio. }\end{array}$ \\
\hline $\begin{array}{l}\text { Mercury } \\
\text { amalgamation / } \\
\text { HG-AMALG } \\
\text { (MOXER) }\end{array}$ & 68 & - & $\begin{array}{l}\text { Amalgamation of mercury wastes. Addition of } \\
\text { copper in ratio of } 0.7: 1 \text { (Cu:waste) as determined } \\
\text { by FORTRAN block ADDITIVE. }\end{array}$ \\
\hline $\begin{array}{l}\text { Metal } \\
\text { decontamination / } \\
\text { MET-DCON } \\
\text { (SEP) }\end{array}$ & 64 & - & $\begin{array}{l}\text { Decontamination of metal with abrasive blasting. } \\
\text { Assumes water input in a ratio of } 112 \mathrm{lb} \text { per } 468 \\
\mathrm{lb} \text { waste. Assumes all grit recycled (no make-up } \\
\text { grit added to process). Liquid stream }\left(\mathrm{H}_{2} \mathrm{O}, \mathrm{H}_{2} \text {, }\right. \\
\left.\mathrm{C}, \mathrm{Al}_{2} \mathrm{O}_{3} \text {, and } \mathrm{SiO}_{2}\right) \text { is sent to aqueous waste } \\
\text { treatment. Clean metal }(\mathrm{Fe} \text { ) exits the process. }\end{array}$ \\
\hline $\begin{array}{l}\text { Organic } \\
\text { destruction / } \\
\text { CWO-1 } \\
\text { (RSTOIC) }\end{array}$ & 302 & -3252045 & 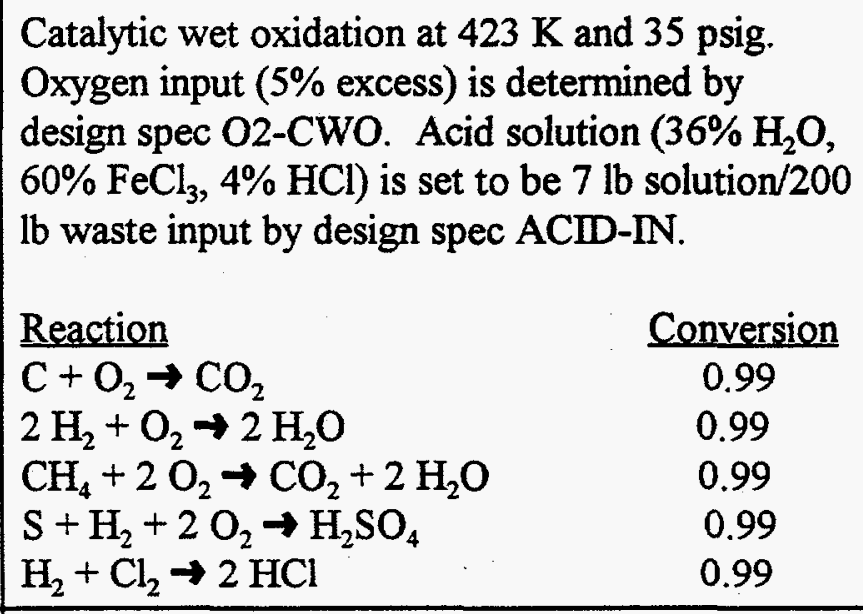 \\
\hline $\begin{array}{l}\text { Organic } \\
\text { destruction filter / } \\
\text { CWOSPLT } \\
\text { (SEP) }\end{array}$ & 299 & - & $\begin{array}{l}\text { Separation of products from CWO reactor. } \\
\text { Solids }\left(\mathrm{S}, \mathrm{Fe}, \mathrm{Ba}, \mathrm{Cd}, \mathrm{Cr}, \mathrm{Fe}(\mathrm{OH})_{3}, \mathrm{C}, \mathrm{SiO} \mathrm{O}_{2} \text {, }\right. \\
\left.\mathrm{Al}_{2} \mathrm{O}_{3}\right) \text { are sent to polymer stabilization. Liquids } \\
\left(\mathrm{H}_{2} \mathrm{O}, \mathrm{HCl}, \mathrm{FeCl} \mathrm{H}_{3}, \mathrm{H}_{2} \mathrm{SO}_{4}\right) \text { are sent for } \\
\text { nutralization. } \mathrm{Gases}\left(\mathrm{O}_{2}, \mathrm{Cl}_{2}, \mathrm{~F}_{2}, \mathrm{CO}_{2}, \mathrm{CH}_{4}\right) \text { are } \\
\text { subject to equilibration and then sent to the APC } \\
\text { subsystem. }\end{array}$ \\
\hline
\end{tabular}




\begin{tabular}{|c|c|c|c|}
\hline $\begin{array}{l}\text { Gas equilibration } \\
\text { / EQULL-1 } \\
\text { (RGIBBS) }\end{array}$ & 302 & -11916 & $\begin{array}{l}\text { This block equilibrates the gas leaving the } \mathrm{CWO} \\
\text { system to account for the non-equilibrium of the } \\
\mathrm{CWO} \text { reactor. It reacts the small amount of } \mathrm{CH}_{4} \\
\text { remaining with } \mathrm{O}_{2} \text { to form } \mathrm{H}_{2} \mathrm{O} \text { and } \mathrm{CO}_{2} . \mathrm{Cl}_{2} \text { is } \\
\text { combined with } \mathrm{H}_{2} \text { to form } \mathrm{HCl} \text {. }\end{array}$ \\
\hline $\begin{array}{l}\text { Neutralization / } \\
\text { NEUTRAL1 } \\
\text { (RSTOIC) }\end{array}$ & 100 & -351812 & 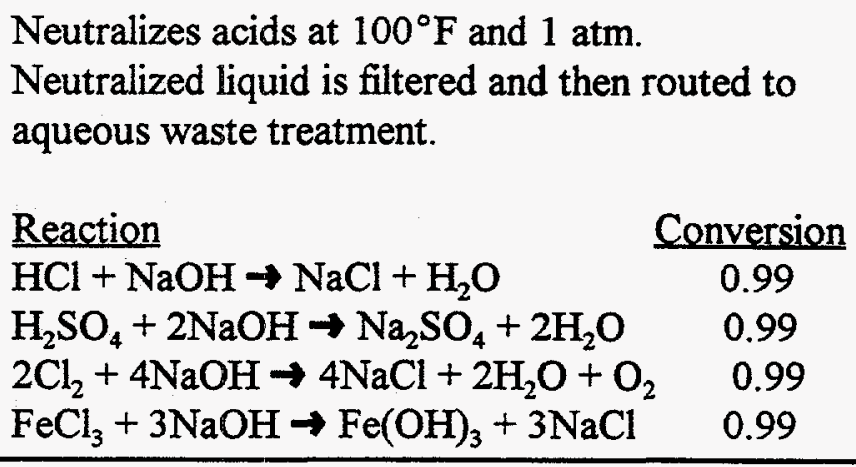 \\
\hline $\begin{array}{l}\text { Precipitation filter } \\
\text { / SALTFLTR }\end{array}$ & 100 & - & $\begin{array}{l}\text { Filters insoluble precipitate } \mathrm{Fe}(\mathrm{OH})_{3} \text { from } \\
\text { aqueous stream. Solid is routed to polymer } \\
\text { stabilization. }\end{array}$ \\
\hline $\begin{array}{l}\text { Lead recovery- } \\
\text { separation / } \\
\text { PB-SPLIT (SEP) }\end{array}$ & 68 & - & $\begin{array}{l}\text { Separates brick or sheet lead (assumed to be } 81 \% \\
\text { of total) for decontamination. Remaining lead } \\
\text { (leaded gloves, blankets, shot) is routed to grout } \\
\text { stabilization. }\end{array}$ \\
\hline $\begin{array}{l}\text { Lead } \\
\text { decontamination / } \\
\text { PB-DECON } \\
\text { (SEP) }\end{array}$ & 63 & - & $\begin{array}{l}\text { Decontaminates lead with water wash, separates } \\
\text { clean lead, which exits the system. Liquid and } \\
\text { sludge are routed to process residue treatment } \\
\text { subsystem. }\end{array}$ \\
\hline $\begin{array}{l}\text { Polymer feed } \\
\text { preparation / } \\
\text { POLY-MIX } \\
\text { (MIXER) }\end{array}$ & 188 & - & Mixes incoming waste for polymer stabilization. \\
\hline $\begin{array}{l}\text { Dryer / DRYER } \\
\text { (HEATER) }\end{array}$ & 200 & 14613 & $\begin{array}{l}\text { Removes excess water prior to polymer } \\
\text { stabilization. }\end{array}$ \\
\hline $\begin{array}{l}\text { Dryer / } \\
\text { WATERSEP } \\
\text { (SEP) }\end{array}$ & 200 & - & $\begin{array}{l}\text { Separates water, input to polymer stabilization is } \\
\text { dried to } 5 \% \text { moisture content, as determined by } \\
\text { design spec DRYER. Excess } \mathrm{H}_{2} \mathrm{O}, \mathrm{NO}, \mathrm{SO}_{2} \text { ) are } \\
\text { sent to aqueous waste treatment subsystem. }\end{array}$ \\
\hline $\begin{array}{l}\text { Mixing / } \\
\text { POLYSTAB } \\
\text { (MIXER) }\end{array}$ & 103 & - & $\begin{array}{l}\text { Adds polyethylene to encapsulate the waste. } \\
\text { Polymer:waste ratio is } 1: 1 \text {. }\end{array}$ \\
\hline
\end{tabular}




\begin{tabular}{|c|c|c|c|}
\hline $\begin{array}{l}\text { Process residue } \\
\text { dryer / } \\
\text { VACTRAX1 } \\
\text { (RGIBBS) }\end{array}$ & 550 & 515629 & $\begin{array}{l}\text { Models the vacuum desorber for process residue } \\
\text { and inorganic sludges. Operates at } 550^{\circ} \mathrm{F} \text { and } 1 \\
\text { psig. Equilibrium is assumed for species entered } \\
\text { in the RGIBBS block: } \mathrm{H}_{2} \mathrm{SO}_{4}, \mathrm{HCl} \text {, and } \mathrm{CH}_{4} \text { are } \\
\text { assumed to form from feed components. } \mathrm{O}_{2} \text { is } \\
\text { excluded from reaction. A nitrogen sweep gas is } \\
\text { used at a rate of } 1.09 \mathrm{ft}^{3} \text { per lb waste }(0.0793 \mathrm{lb} \\
\mathrm{N}_{2} / \mathrm{lb} \text { waste) as determined by FORTRAN block } \\
\text { N2SWEEP. }\end{array}$ \\
\hline $\begin{array}{l}\text { Mercury leaching } \\
\text { / VACSEP-1 } \\
\text { (SEP) }\end{array}$ & 97 & - & $\begin{array}{l}\text { Mercury leaching of process residue and inorganic } \\
\text { sludges by addition of leach water ( } 30 \% \text { of mass } \\
\text { of incoming waste). Also includes separation of } \\
\text { gases, liquids, organics, } \mathrm{Hg} \text {-contaminated liquids, } \\
\text { and solids. } \\
\text { Gases }\left(\mathrm{N}_{2}\right) \text { are sent to } \mathrm{APC} \text { subsystem. Solids } \\
\left(\mathrm{H}_{2} \mathrm{O} \text { in leach water, } \mathrm{S}, 50 \% \text { of } \mathrm{H}_{2}, \mathrm{O}_{2}, 50 \% \text { of C, }\right. \\
\mathrm{SiO}_{2}, \mathrm{Al}_{2} \mathrm{O}_{3}, \mathrm{Ba}, \mathrm{As}, \mathrm{Pb}, \mathrm{Fe}, \mathrm{Cd}, \mathrm{Cr}, \mathrm{Se}, \mathrm{Ag} \text { ) are } \\
\text { routed to polymer stabilization subsystem. Liquids } \\
\left.\text { (balance of } \mathrm{H}_{2} \mathrm{O}, \mathrm{HCl}, \mathrm{H}_{2} \mathrm{SO}\right) \text { are sent to aqueous } \\
\text { waste treatment. Organics ( } 50 \% \text { of } \mathrm{H}_{2}, 50 \% \text { of } \\
\mathrm{C}, \mathrm{CH})_{4} \text { are routed to the organic destruction } \\
\text { subsystem. Mercury separated is routed to } \\
\text { mercury amalgamation. }\end{array}$ \\
\hline $\begin{array}{l}\text { Open debris wash } \\
\text { / HP-WASH } \\
\text { (SEP) }\end{array}$ & 66 & - & $\begin{array}{l}\text { Models the aqueous wash for open debris. Make- } \\
\text { up water of } 15 \% \text { of mass of incoming waste is } \\
\text { used. Two-thirds of the make-up water will be } \\
\text { routed to aqueous waste treatment; one-third of } \\
\text { the water is assumed to be entrained with the } \\
\text { waste after washing. The washing process } \\
\text { produces an aqueous stream ( } 98 \% \text { of } \mathrm{O}_{2}, 98 \% \text { of } \\
\mathrm{H}_{2}, 98 \% \text { of } \mathrm{C} \text {, two-thirds of the wash water, } 1 \% \\
\text { of } \mathrm{SiO}_{2} \text {, and } 1 \% \text { of } \mathrm{Al}_{2} \mathrm{O}_{3} \text { ) that is sent to aqueous } \\
\text { waste treatment. The remaining solids } \\
\text { (remainder of } \mathrm{H}_{2} \mathrm{O}, 2 \% \text { of } \mathrm{O}_{2}, 2 \% \text { of } \mathrm{H}_{2}, \mathrm{~N}_{2}, \mathrm{~S} \text {, } \\
2 \% \text { of } \mathrm{Cl}_{2}, \mathrm{Fe}, \mathrm{As}, \mathrm{Ba}, \mathrm{Cd}, \mathrm{Cr}, \mathrm{Pb}, \mathrm{Hg}_{\mathrm{S}} \mathrm{Se}, \mathrm{Ag} \text {, } \\
2 \% \text { of C, } 99 \% \text { of } \mathrm{SiO}_{2} \text {, and } 99 \% \text { of } \mathrm{Al}_{2} \mathrm{O}_{3} \text { ) are } \\
\text { routed to the grout stabilization subsystem. }\end{array}$ \\
\hline
\end{tabular}




\begin{tabular}{|c|c|c|c|}
\hline $\begin{array}{l}\text { Soft debris } \\
\text { destruction / } \\
\text { CWO-2 } \\
\text { (RSTOIC) }\end{array}$ & 302 & -4625466 & 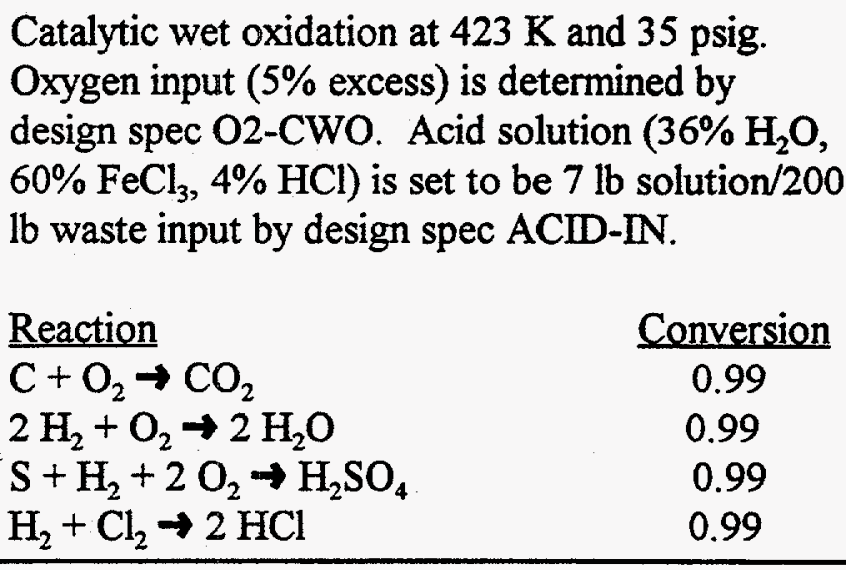 \\
\hline $\begin{array}{l}\text { Organic } \\
\text { destruction filter / } \\
\text { CWOSPL2 (SEP) }\end{array}$ & 299 & - & $\begin{array}{l}\text { Separation of products from } \mathrm{CWO} \text { reactor. } \\
\text { Solids }\left(\mathrm{O}_{2}, \mathrm{~S}, \mathrm{Fe}, 95 \% \text { of } \mathrm{Hg}, \mathrm{Fe}(\mathrm{OH})_{3}, \mathrm{C}, \mathrm{SiO}_{2} \text {, }\right. \\
\left.\mathrm{Al}_{2} \mathrm{O}_{3}\right) \text { are sent to grout stabilization. } \mathrm{Liquids} \\
\left(\mathrm{H}_{2} \mathrm{O}, 5 \% \text { of } \mathrm{Hg}, \mathrm{HCl}, \mathrm{FeCl}_{3}, \mathrm{H}_{2} \mathrm{SO}_{4}, \mathrm{Na}_{2} \mathrm{SO}_{4},\right. \\
\mathrm{NaOH} \text {, and } \mathrm{NaCl}) \text { are sent for neutralization. } \\
\left.\mathrm{Gases} \mathrm{N}_{2}, \mathrm{Cl}_{2}, \mathrm{~F}_{2}, \mathrm{CO}_{2}\right) \text { are subject to } \\
\text { equilibration and then sent to the APC subsystem. }\end{array}$ \\
\hline $\begin{array}{l}\text { Gas equilibration } \\
\text { / EQUIL-2 } \\
\text { (RGIBBS) }\end{array}$ & 302 & 506 & $\begin{array}{l}\text { This block equilibrates the gas leaving the CWO } \\
\text { system to account for possible non-equilibrium of } \\
\text { the CWO reactor. In this case, it only serves as a } \\
\text { heater to raise the temperature to } 302^{\circ} \mathrm{F} \text {. }\end{array}$ \\
\hline $\begin{array}{l}\text { Neutralization / } \\
\text { NEUTRAL2 } \\
\text { (RSTOIC) }\end{array}$ & 100 & -388029 & 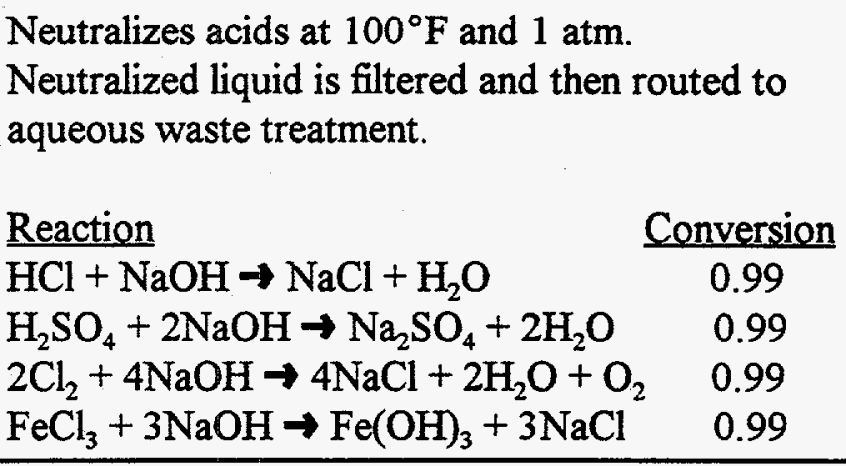 \\
\hline $\begin{array}{l}\text { Precipitation filter } \\
\text { / SALTFLTR }\end{array}$ & 100 & - & $\begin{array}{l}\operatorname{Removes} \mathrm{Fe}(\mathrm{OH})_{3} \text { from aqueous stream. Solid is } \\
\text { routed to polymer stabilization. }\end{array}$ \\
\hline Special waste & 68 & - & $\begin{array}{l}\text { As with the ITTS study, no model was } \\
\text { constructed for special waste. }\end{array}$ \\
\hline
\end{tabular}




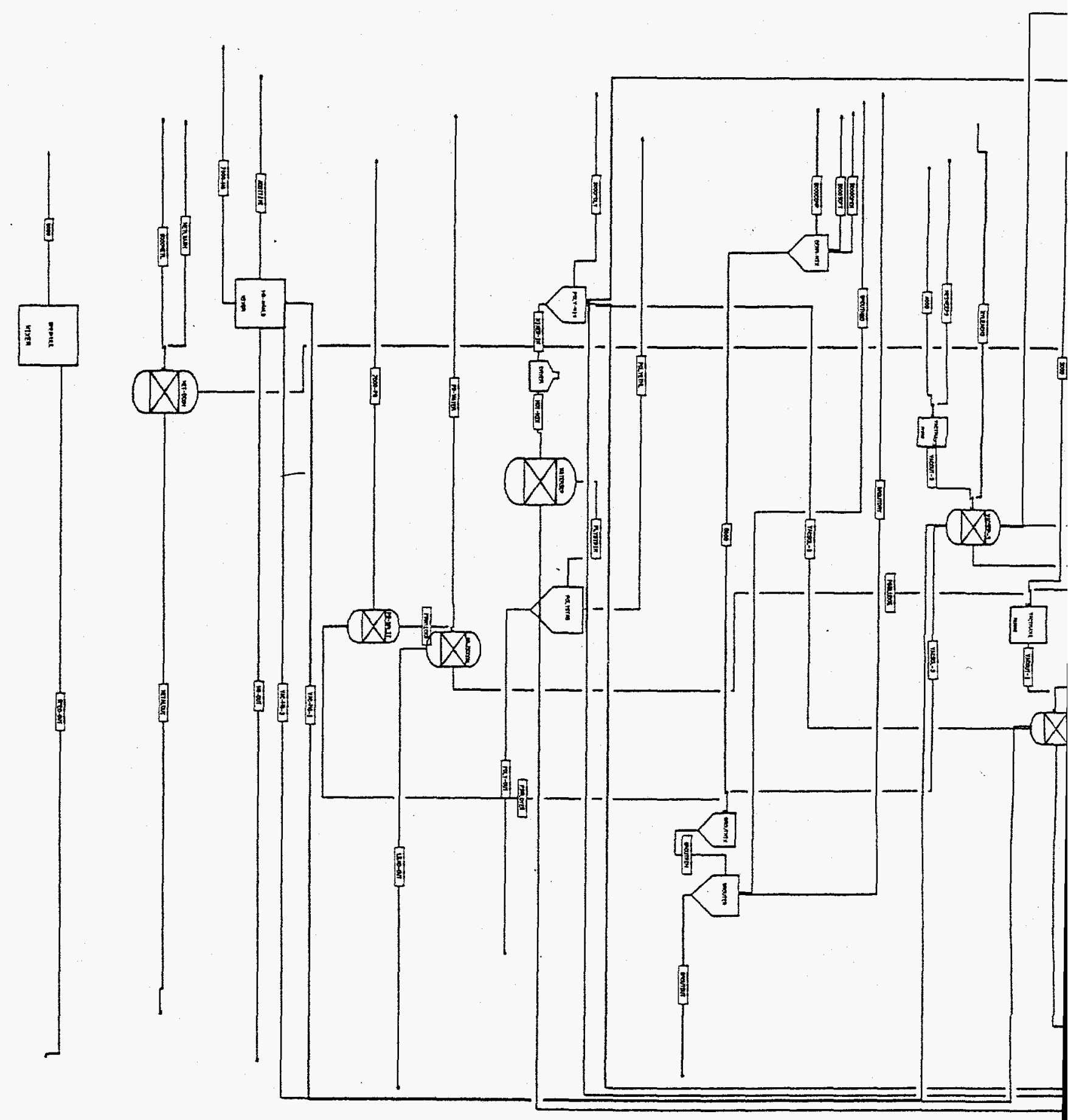

INTS System 1 


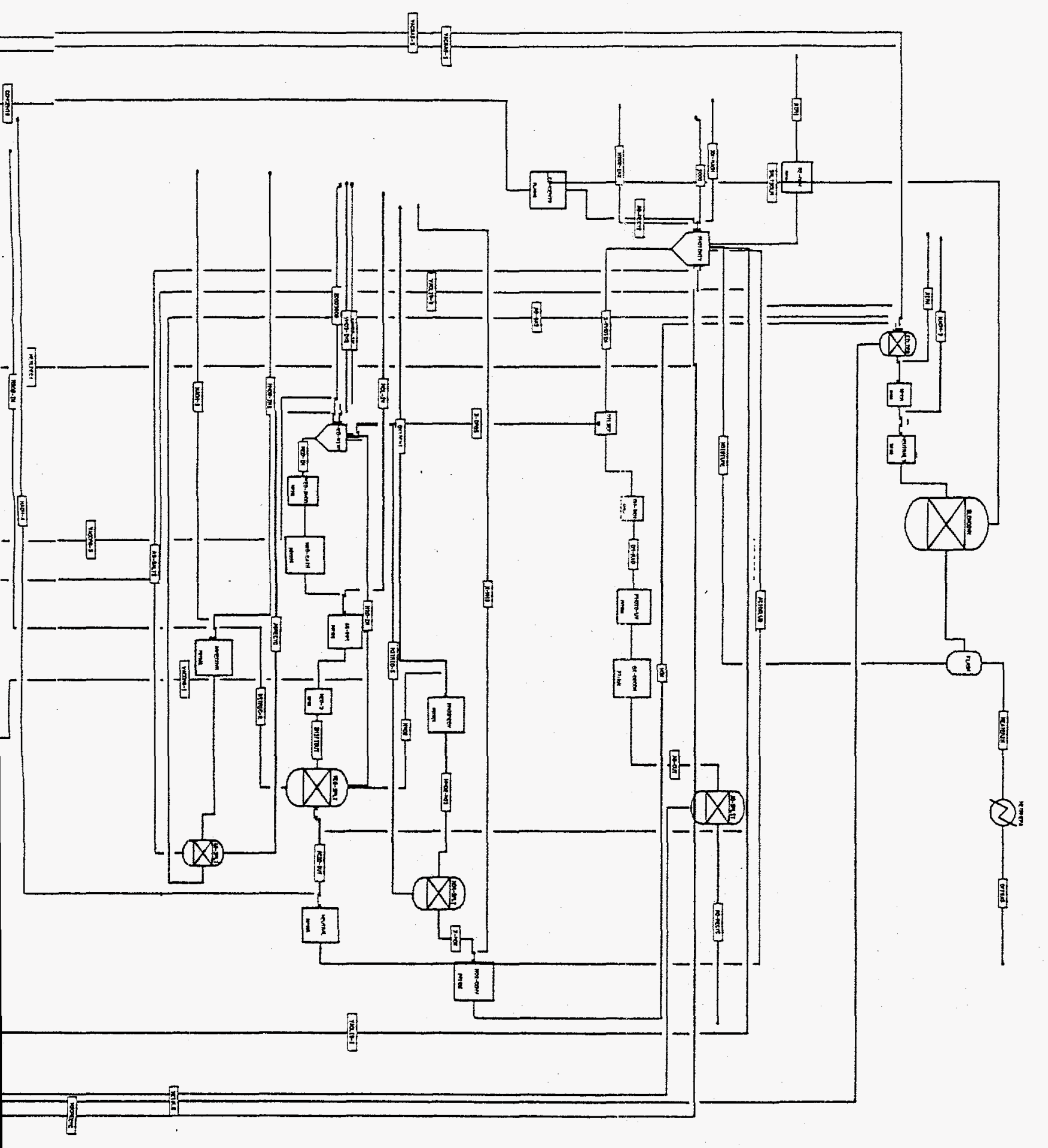




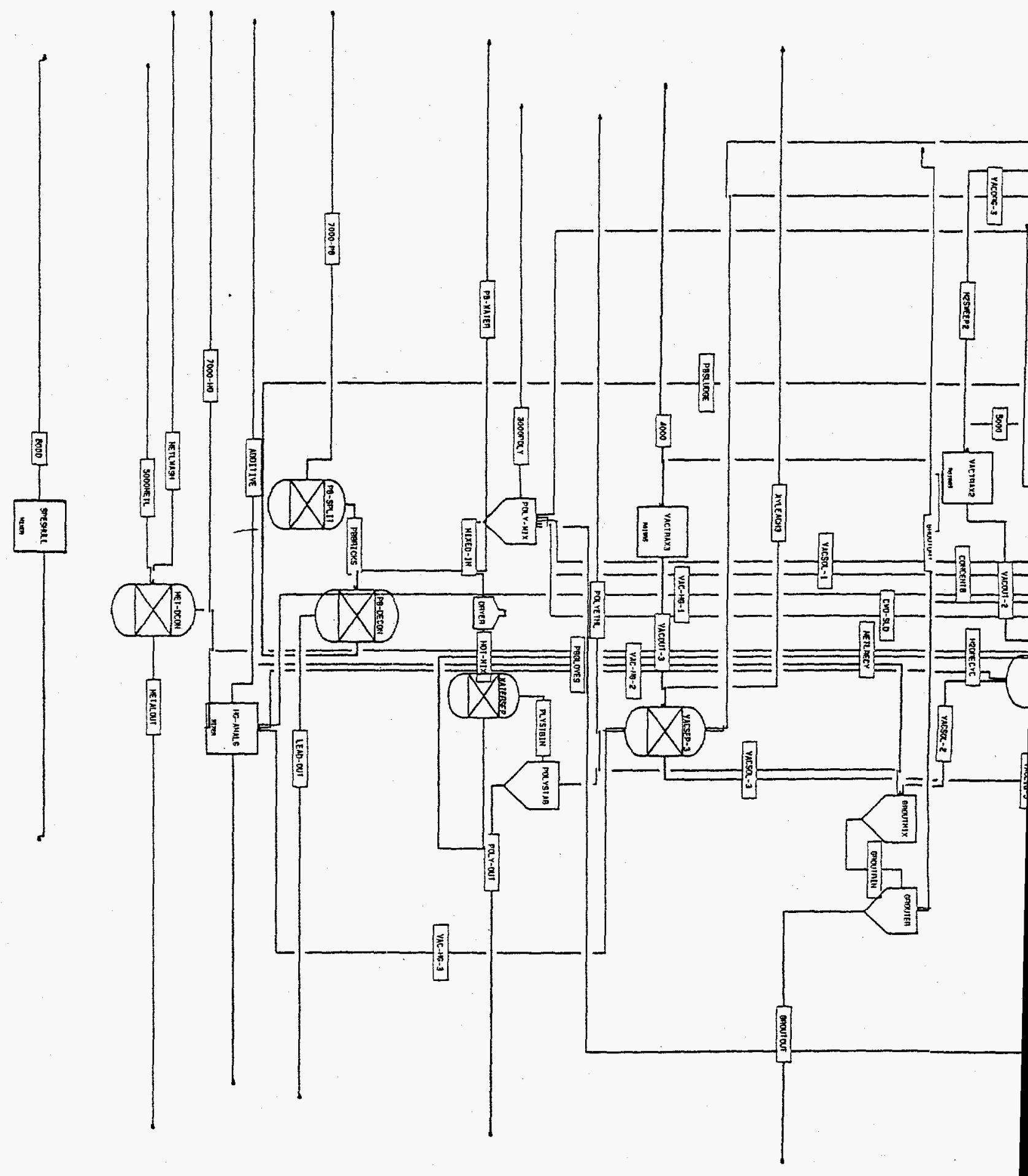

INTS System 2 


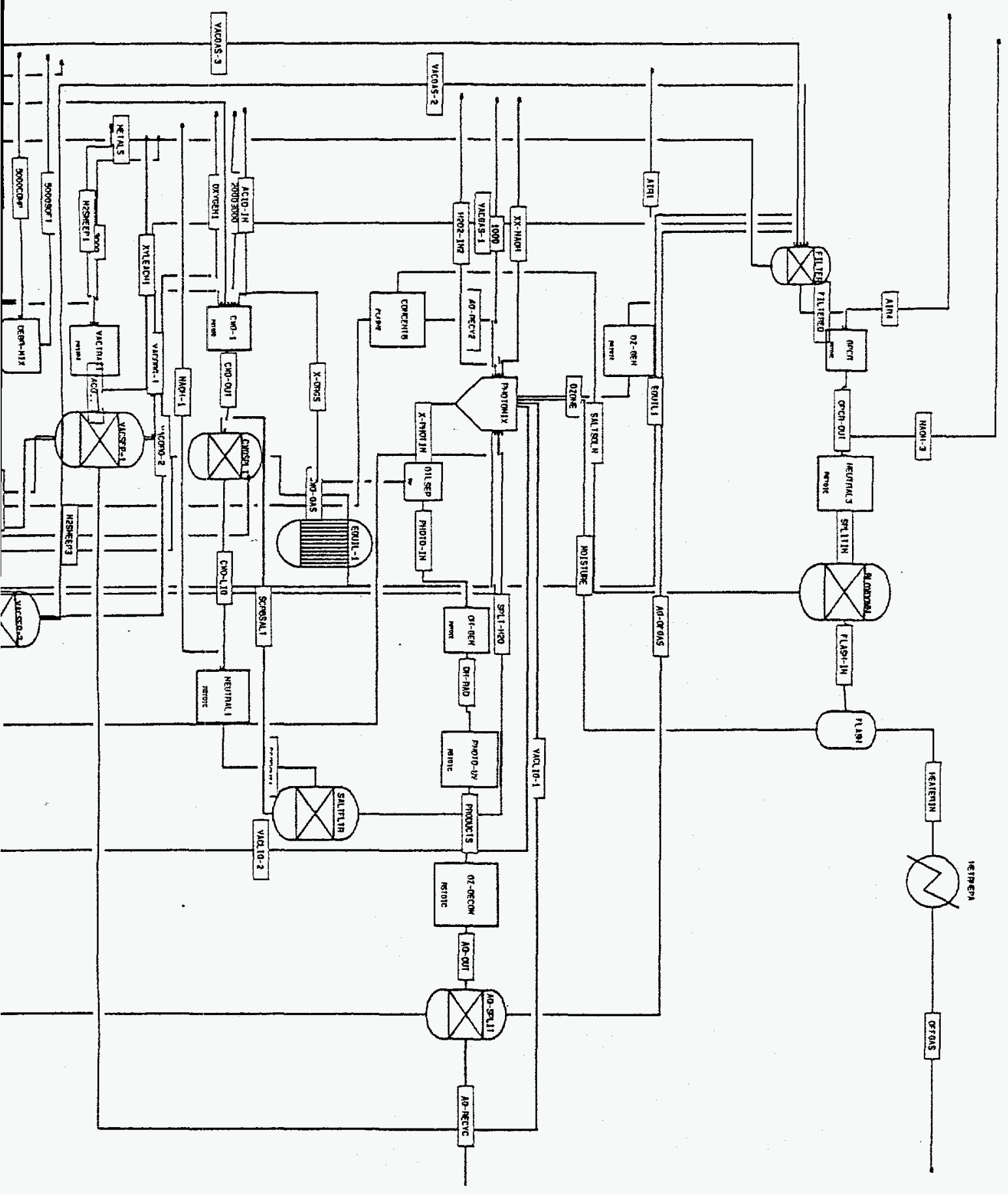




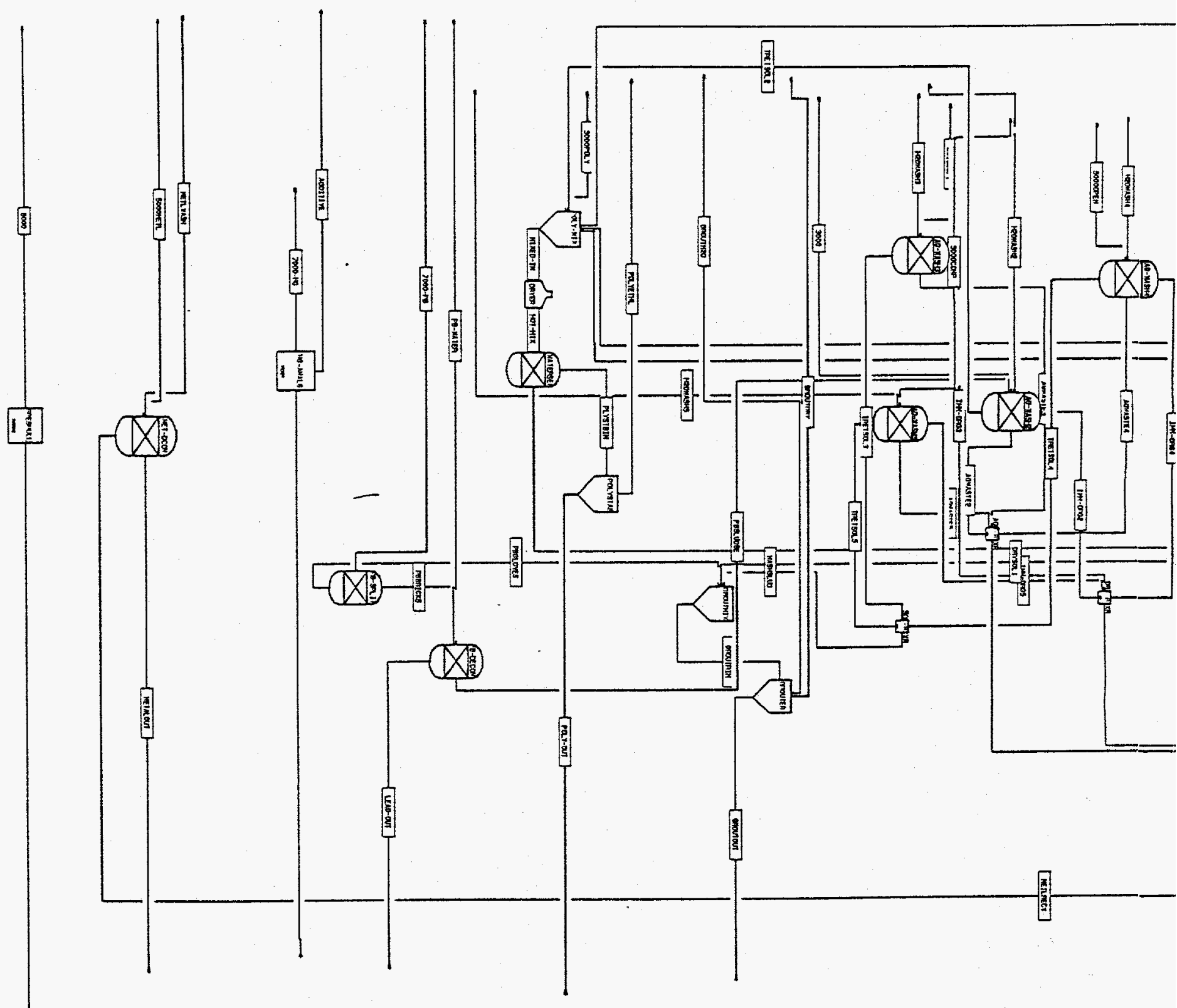

INTS System 3 


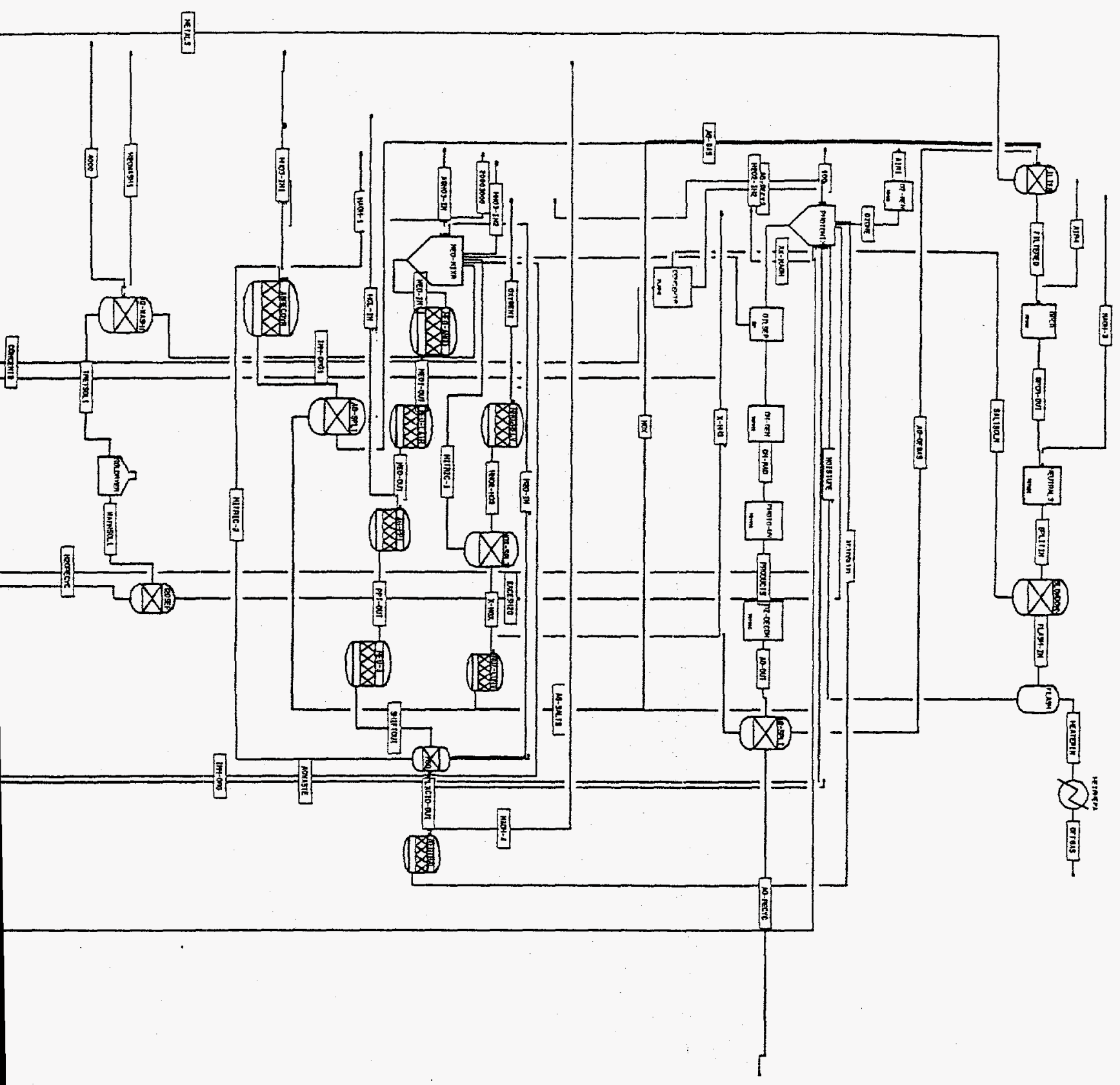




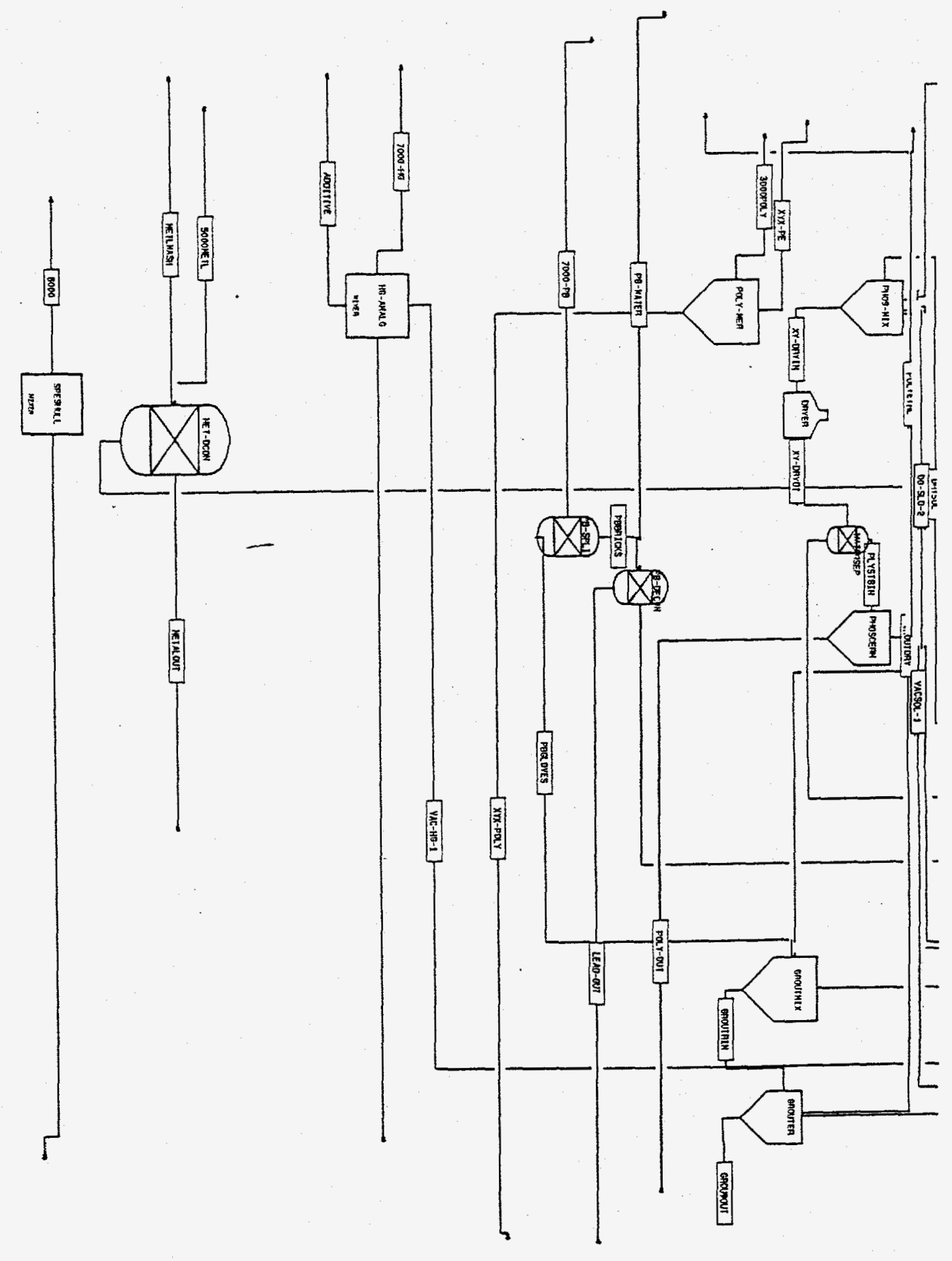

INTS System 4 


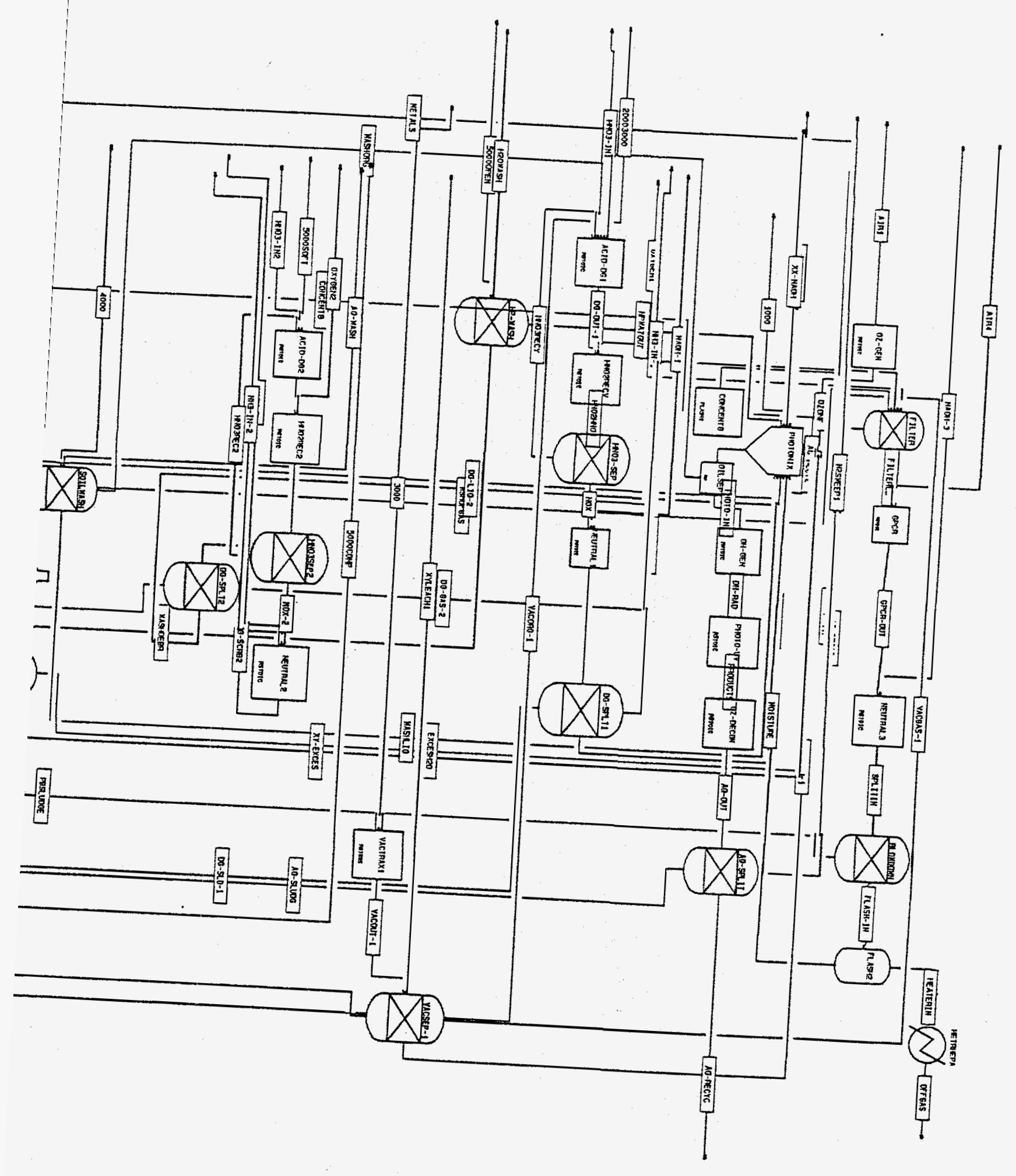




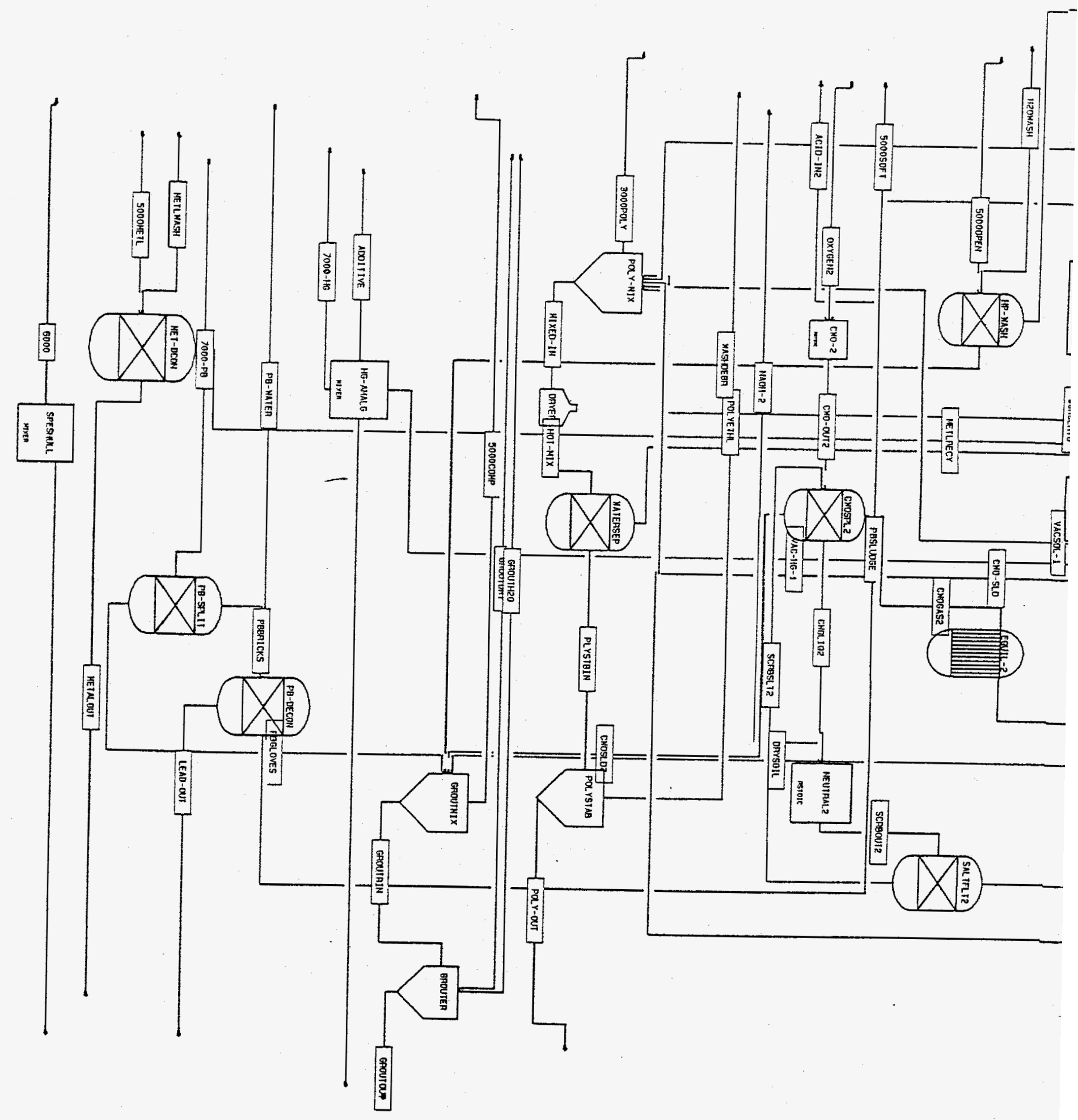

INTS System 5 


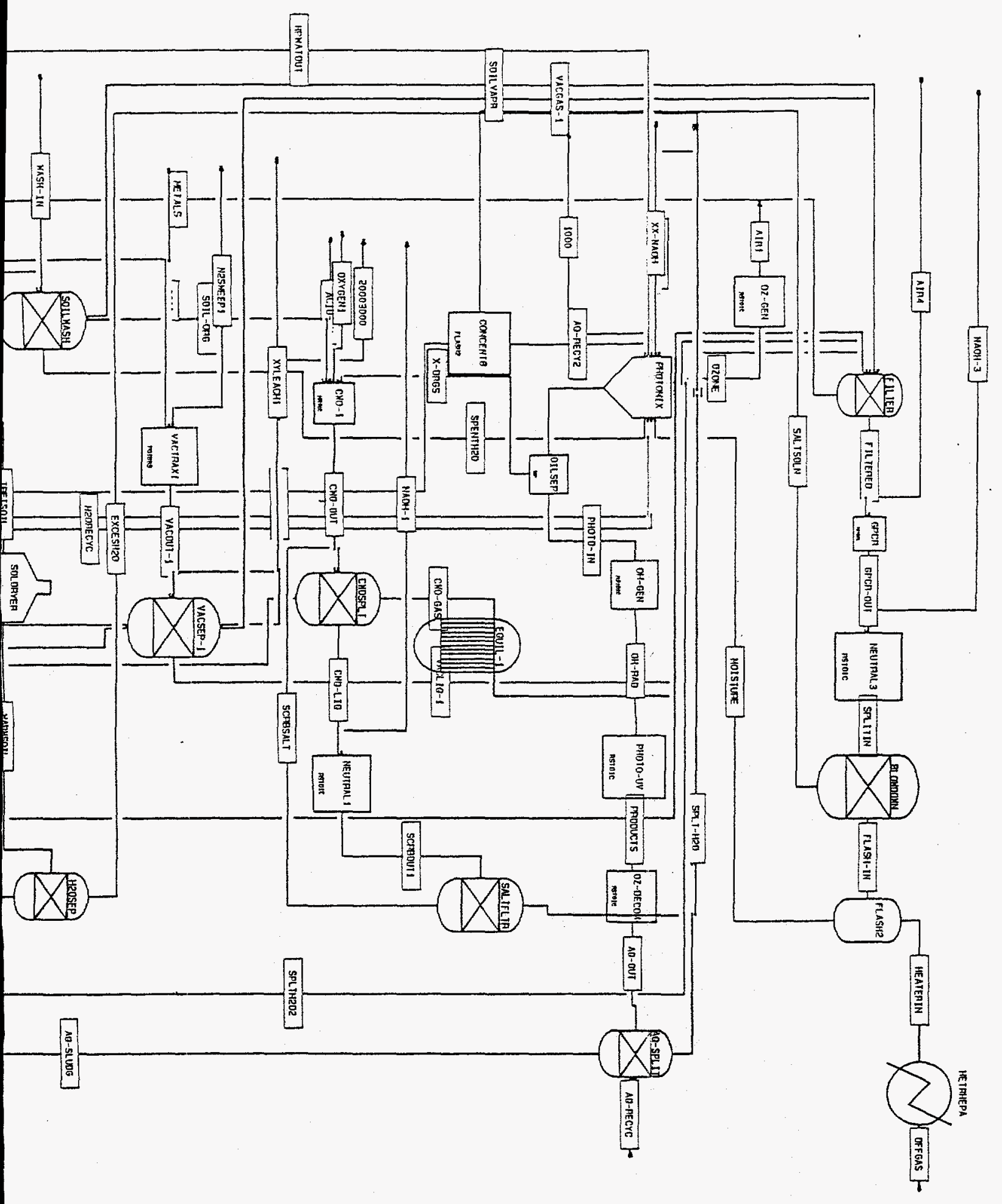


Appendix B

ASPEN PLUS Input Files 


\section{ASPEN PLUS Input Files}

The following input files are can be entered as .INP (input) files to ASPEN PLUS to reproduce the results obtained herein. Convergence may be difficult, if not impossible, with the code as listed below; it may be necessary to remove and then successively reintroduce the design specifications from the model (using the HIDE and REVEAL features of Model Manager, or by commenting out the coded lines) in order to obtain converged runs. 


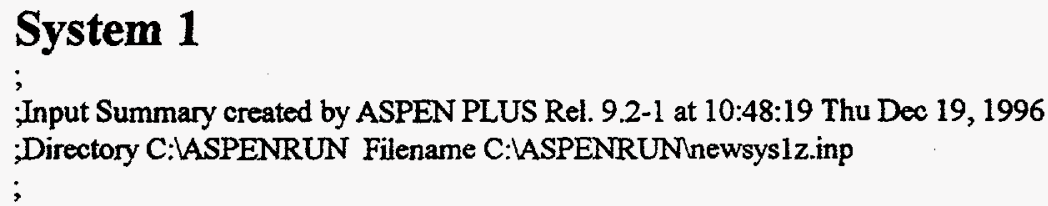

PROP-SOURCES COMBUST / INORGANIC / PURECOMP / SOLDS / \& AQUEOUS / PURE856 / ASPENPCD

;Cement approximated by CaAl2SiO6 (CaOAl2O3.SiO2), Shreve \& Brink p. 162

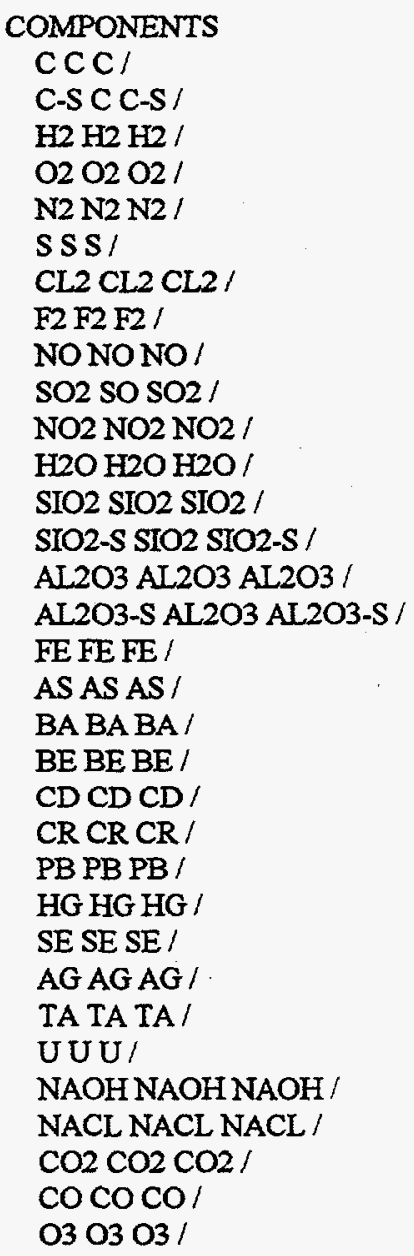


HCL HCL HCL /

FECL3 FECL3 FECL3/

$\mathrm{H} 2 \mathrm{SO} 4 \mathrm{H}_{2} \mathrm{SO} 4 \mathrm{H}_{2} \mathrm{SO} 4 /$

NA2SO4 NA2SO4 NA2SO4 /

$\mathrm{CH} 4 \mathrm{CH} 4 \mathrm{CH} 4$ /

POLYMER C2H4 POLYMER /

CEMENT CAAL2SIO6 CEMENT /

CASO4 CASO4 CASO4/

AGNO3 AGNO3 AGNO3/

HNO3 HNO3 HNO3/

$\mathrm{HNO} 2 \mathrm{HNO} 2 \mathrm{HNO} 2 /$

NO3-NO3-NO3- 1

$\mathrm{H}+\mathrm{H}+\mathrm{H}+1$

E-E-E-

$\mathrm{AG}+\mathrm{AG}+\mathrm{AG}+1$

$\mathrm{AG}++\mathrm{AG}+2 \mathrm{AG}++1$

$\mathrm{H} 2 \mathrm{O} 2 \mathrm{H}_{2} \mathrm{O} 2 \mathrm{H} 2 \mathrm{O} 2$ /

AGCL AGCL AGCL/

CL- CL- CL- /

CUCUCU/

OH-RAD OH OH-RAD /

NH3 H3N NH3 !

NANO3 NANO3 NANO3

FLOWSHEET APC-SYS

BLOCK GPCR IN=FIL TERED AIR4 OUT=GPCR-OUT

BLOCK FLTER IN=AQ-OFGAS VACGAS-1 VACGAS-3 AG-GAS NOX \& OUT=FILTERED METALS

BLOCK NEUTRAL3 IN=NAOH-3 GPCR-OUT OUT=SPLITN

BLOCK BLOWDOWN IN=SPLITIN OUT=FLASH-IN SALTSOLN

BLOCK FLASH IN=FLASH-IN OUT=HEATERIN MOISTURE

BLOCK HETRHEPA IN=HEATERIN OUT=OFFGAS

FLOWSHEET AQUEOUS

BLOCK AQ-SPLIT IN=AQ-OUT OUT=AQ-SLUDG AQ-OFGAS AQ-RECYC

BLOCK PHOTO-UV $\mathrm{N}=\mathrm{OH}-\mathrm{RAD}$ OUT $=$ PRODUCTS

BLOCK OZ-GEN IN=AIRI OUT=OZONE

BLOCK OZ-DECOM IN=PRODUCTS OUT $=A Q-O U T$

BLOCK CONCENT8 IN=SALTSOLN OUT $=A Q-R E C Y 2$ CONCENT 8

BLOCK PHOTOMIX IN=VACLIQ-1 METLRECY 1000 VACLIQ-3 H2ORECYC \& OZONE MEO-LIQ AG-SALTS MOISTURE H2O2-IN2 ACIDSLUD \& $X X-N A O H$ AQ-RECY2 OUT $=X-P H O T I N$

BLOCK OH-GEN IN $=$ PHOTO-IN OUT $=O H-R A D$

BLOCK OILSEP IN=X-PHOTIN OUT=PHOTO-IN X-ORGS

FLOWSHEET BULKSOIL

BLOCK VACSEP-3 IN=VACOUT-3 XYLEACH 3 OUT=VACLIQ-3 VACGAS-3 \&

VACSOL-3 VACORG-3 VAC-HG-3

BLOCK VACTRAX $3 \mathrm{IN}=4000$ N2SWEEP3 OUT=VACOUT -3

FLOWSHEET GROUT

BLOCK GROUTMIX IN=PBGLOVES VACSOL-3 5000 OUT=GROUTRIN

BLOCK GROUTER IN=GROUTDRY GROUTRIN GROUTH2O OUT=GROUTOUT

BLOCK DEBR-MIX IN=5000COMP 5000SOFT 5000OPEN OUT $=5000$

FLOWSHEET HG-AMAL

BLOCK HG-AMALG $\mathbb{N}=$ =VAC-HG-3 VAC-HG-1 ADDITIVE 7000-HG OUT $=$ \& HG-OUT 


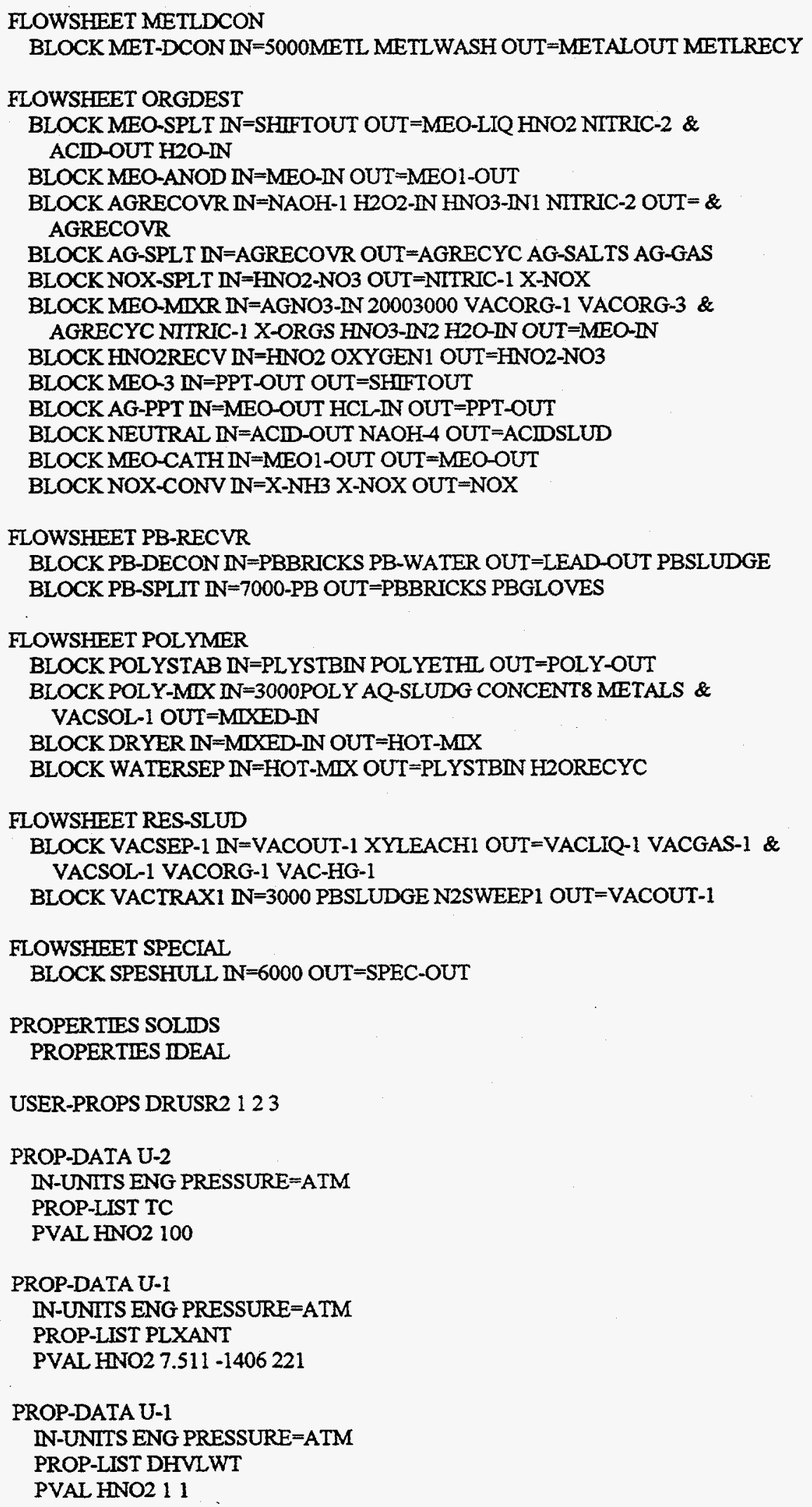


;Tables dated 04/24/96 from Daryoush Bahar, MK, (415) 442-7688

;Glass inerts assumed to be $50 \% \mathrm{SiO} 2$ and $50 \% \mathrm{Al} 2 \mathrm{O} 3$ for all waste streams.

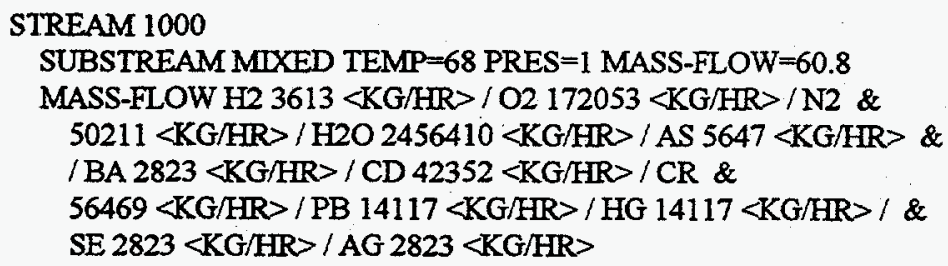

;Tables dated 04/24/96 from MK

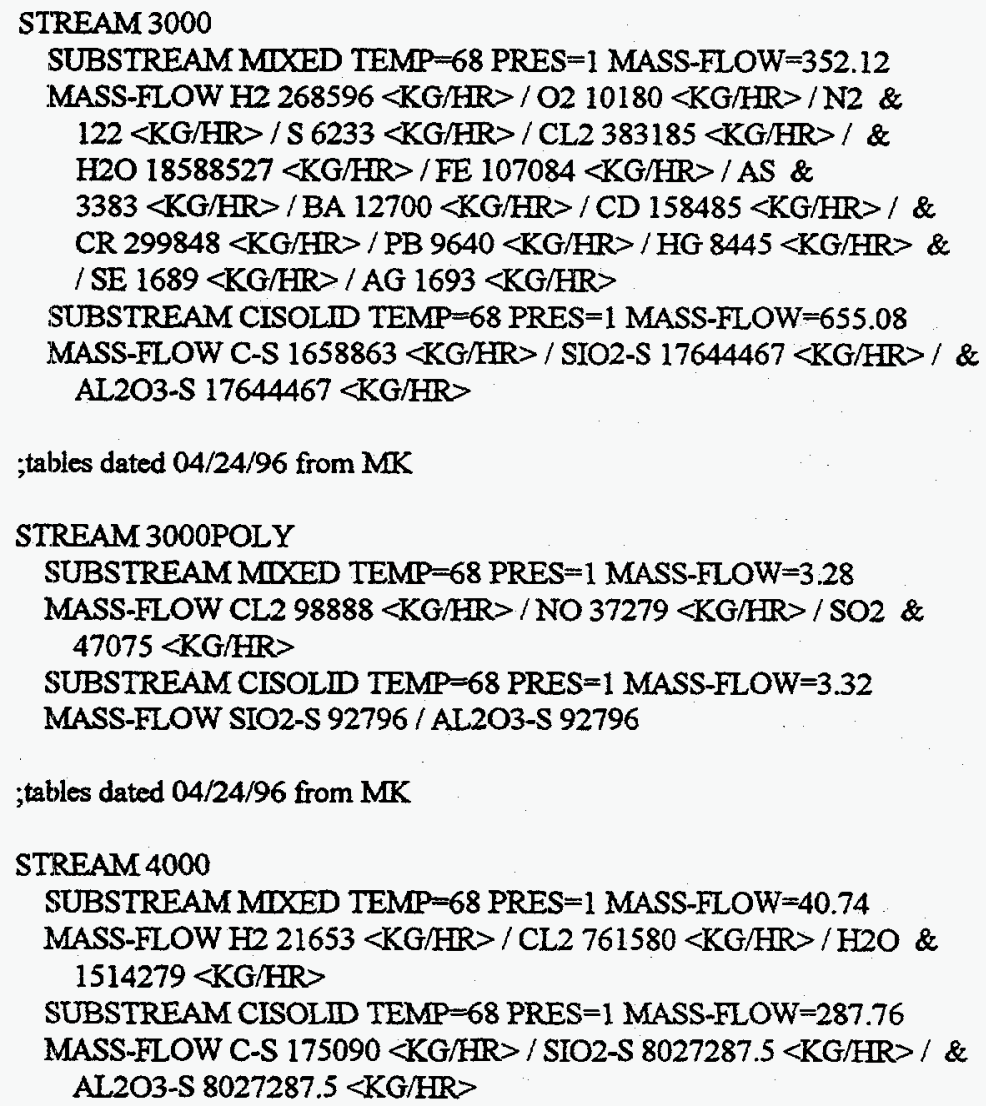

;tables dated 04/24/96 from MK

Data table dated 04/24/96. Supplied by Daryoush Bahar, MIK (415) 442-7688

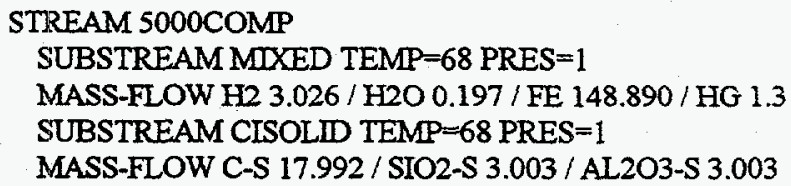

; tables dated 04/24/96 from MK

STREAM 5000METL

SURSTREAM MIXED TEMP $=68$ PRES $=1$ MASS-FLOW=434.82

MASS-FLOW H2 141789 / FE 13383118 
$1229766<\mathrm{KG} / \mathrm{HR}>/$ FE $325702<\mathrm{KG} / \mathrm{HR}>/ \mathrm{BA} 32570<\mathrm{KG} / \mathrm{HR}>$ \& / CD $32570<\mathrm{KG} / \mathrm{HR}>$ / CR $65140<\mathrm{KG} / \mathrm{HR}>$

SUBSTREAM CISOLID TEMP=68 PRES $=1$ MASS-FLOW=107.12 \& FLASH-OPTION $=$ NOFLASH

MASS-FLOW C-S $5723044<$ KG/HR $>$ / SIO2-S $103248<$ KG/HR $>$ / \& AL 203-S $103248<\mathrm{KG} / \mathrm{HR}>$

STREAM ADDITIVE SUBSTREAM MIXED TEMP $=68$ PRES $=1$

MASS-FLOW CU 1.8548

STREAM AGNO3-N

SUBSTREAM MIXED TEMP=68 PRES=1 MASS-FLOW=2.5

MASS-FRAC AGNO3 1

STREAM AGRECYC

SUBSTREAM MIXED TEMP=100 PRES $=1$ MASS-FLOW $=43861$

MASS-FRAC HNO3 1

STREAM AIRI

SUBSTREAM MIXED TEMP=68 PRES=1 MASS-FLOW=5200

MOLE-FRAC O2 $0.21 / \mathrm{N} 20.79$

STREAM AIR4

SUBSTREAM MIXED TEMP $=68$ PRES $=1$ MASS-FLOW $=0.001$

MOLE-FRAC O2 $0.21 /$ N2 0.79

STREAM GROUTDRY

SUBSTREAM MIXED TEMP $=68$ PRES $=1$

SUBSTREAM CISOLID TEMP $=68$ PRES $=1$ MASS-FLOW $=1$

MASS-FRAC CEMENT 1

STREAM GROUTH2O

SUBSTREAM MDXED TEMP $=68$ PRES $=1$ MASS-FLOW $=.00001$

MASS-FRAC $\mathrm{H} 2 \mathrm{O} 1$

STREAM H2O-N

SUBSTREAM MIXED TEMP $=68$ PRES $=1$ MASS-FLOW $=0.000001$

MASS-FRAC $\mathrm{H}_{2} \mathrm{O}_{1}$

STREAM H2O2-IN

SUBSTREAM MIXED TEMP $=68$ PRES $=1$ MASS-FLOW=1

MASS-FRAC $\mathrm{H} 2 \mathrm{O} 21$

STREAM H2O2-IN2̀

SUBSTREAM MIXED TEMP=68 PRES=1 MOLE-FLOW=1

MASS-FRAC $\mathrm{H} 2 \mathrm{O} 21$

STREAM HCL-IN

SUBSTREAM MTXED TEMP $=68$ PRES $=1$ MASS-FLOW $=0.00000001$

MASS-FRAC HCL 1

STREAM HG-OUT

SUBSTREAM MIXED TEMP $=68$ PRES $=1$

MASS-FLOW HG 2.64 / CU 1.8548

STREAM FNO3-IN1

SUBSTREAM MIXED TEMP=68 PRES=1 MASS-FLOW=1000

B-8 


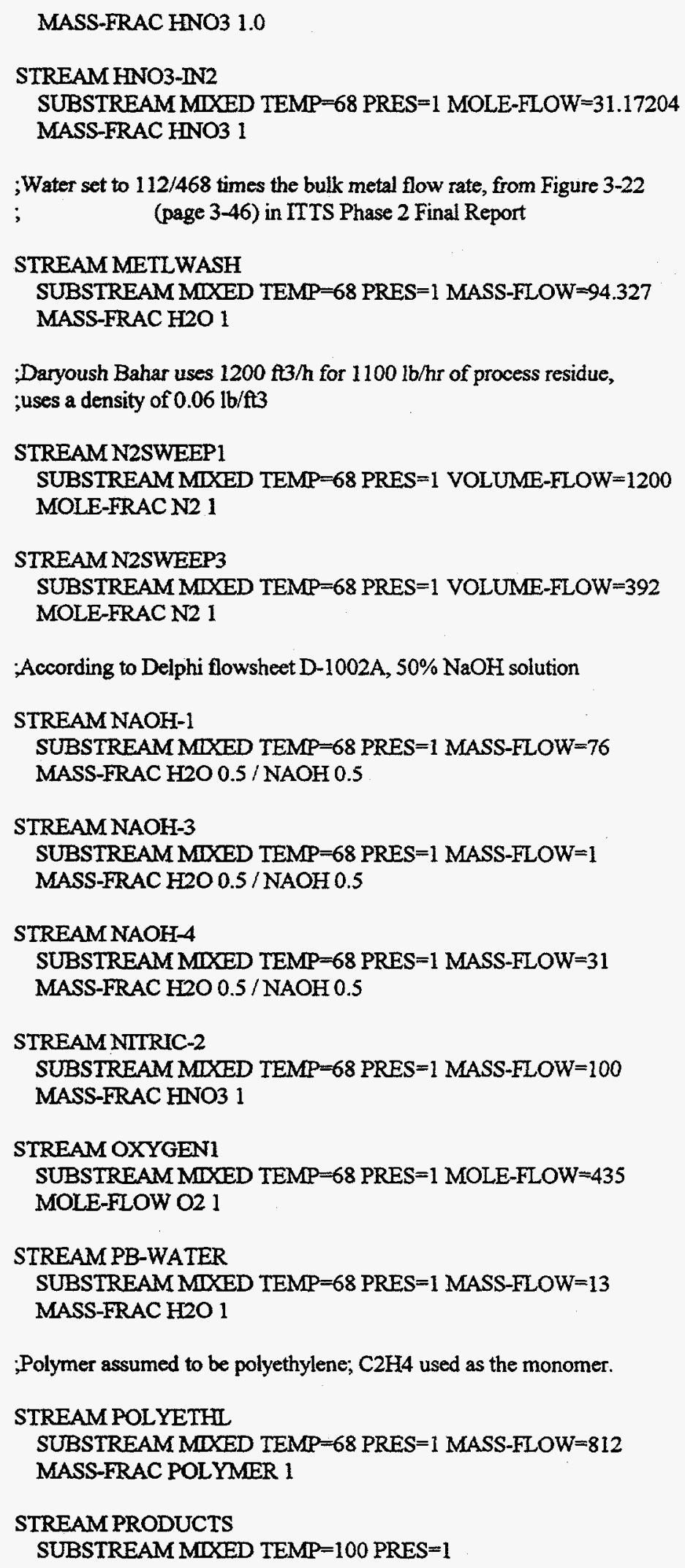

;Polymer assumed to be polyethylene; $\mathrm{C} 2 \mathrm{H} 4$ used as the monomer.

STREAM POLYETHL SUBSTREAM MIXED TEMP=68 PRES $=1$ MASS-FLOW $=812$ MASS-FRAC POLYMER 1

STREAM PRODUCTS SUBSTREAM MIXED TEMP=100 PRES=1 


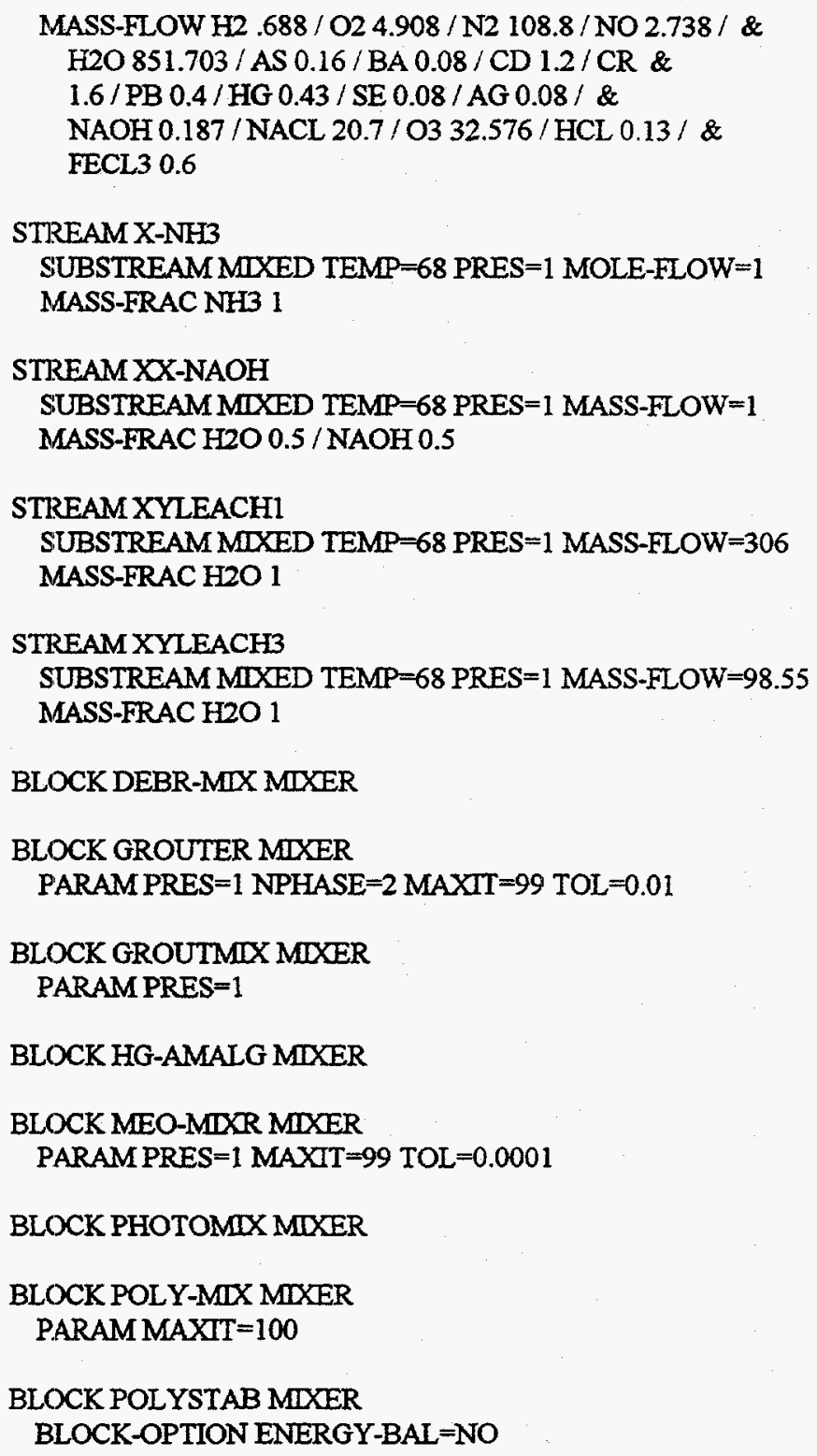


S CL2 F2 NO H2O SIO2 SIO2-S AL2O3 AL2O3-S FE AS BA \& CD CR PB HG SE AG NAOH NACL CO2 $\mathrm{CO} O 3$ HCL FECL 3 \& $\mathrm{H} 2 \mathrm{SO} 4 \mathrm{NA} 2 \mathrm{SO} 4 \mathrm{CH} 4 \mathrm{POLYMER}$ CEMENT CASO4 AGNO3 $\mathrm{HNO} 3 \mathrm{HNO} 2$ \& $\mathrm{NO} 3-\mathrm{H}+\mathrm{E}-\mathrm{AG}+\mathrm{AG}+\mathrm{H} 2 \mathrm{O} 2 \mathrm{AGCL}$ FRACS $=001110$ \& $110000000000000000011 \&$ 1000010000000000000

FRAC STREAM=AG-GAS SUBSTREAM $=$ CISOLID COMPS $=$ C-S SIO2-S \& AL2O3-S CEMENT FRACS $=0000$

FLASH-SPECS AGRECYC TEMP=100 PRES $=1$

FLASH-SPECS AG-SALTS TEMP $=100$ PRES $=1$ NPHASE $=1$

FLASH-SPECS AG-GAS TEMP $=100$ PRES $=1$

BLOCK AQ-SPLIT SEP

DESCRIPTION "this separates water, gases, and solids"

FRAC STREAM=AQ-SLUDG SUBSTREAM=MIXED COMPS=C C-S H2 O2 \& N2 S CL2 F2 NO SO2 H2O SIO2 SIO2-S AL2O3 AL2O3-S \&

FE AS BA CD CR PB HG SE AG NAOH NACL $\mathrm{CO} 2 \mathrm{CO} O 3$ \& HCL FECL3 H2SO4 NA2SO4 CH4 POLYMER CEMENT CASO4 AGNO3 \& HNO3 HNO2 NO3- $\mathrm{H}+\mathrm{E}-\mathrm{AG}+\mathrm{AG}+\mathrm{H} 2 \mathrm{O} 2$ AGCL CL-CU \&

FRACS $=11000110000000.990 .990 .99$ \&

0.991111111111100001010 \&

111100000000101

FRAC STREAM=AQ-SLUDG SUBSTREAM=CISOLID COMPS=C-S SIO2-S \& AL2O3-S CEMENT FRACS $=0.990 .990 .990 .99$

FRAC STREAM=AQ-OFGAS SUBSTREAM=MIXED COMPS $=C$ C-S H2 O2 \& N2 S CL2 F2 NO SO2 NO2 H2O SIO2 SIO2-S AL2O3 \&

AL2O3-S FE AS BA CD CR PB HG SE AG NAOH NACL CO2 \& CO O3 HCL FECL3 H2SO4 NA2SO4 CH4 POLYMER CEMENT \& CASO4 FRACS $=0011101110100000 \&$ $000000000001110000100 \&$ 0

FRAC STREAM=AQ-OFGAS SUBSTREAM=CISOLID COMPS=C-S SIO2-S \& AL2O3-S CEMENT FRACS $=0000$

BLOCK BLOWDOWN SEP

FRAC STREAM=SALTSOLN SUBSTREAM=MIXED COMPS=C C-S H2 O2 \& N2 S CL2 $\mathrm{F} 2$ NO SO2 NO2 H2O SIO2 SIO2-S AL2O3 \& AL203-S FE AS BA BE CD CR PB HG SE AG TA UNAOH \& NACL CO2 $\mathrm{CO} O 3 \mathrm{HCL}$ FECL3 H2SO4 NA2SO4 CH4 POLYMER \& CEMENT CASO4 AGNO3 HNO3 HNO2 NO3-H+E-AG+AG++ \& H2O2 AGCL CL- CU OH-RAD NH3 NANO3 FRACS $=000000$ \& $0000000.20000000000000 \&$ $000110000001000000000 \&$ 0000000001

FRAC STREAM=SALTSOLN SUBSTREAM=CISOLD COMPS=C-S SIO2-S \& AL2O3-S CEMENT FRACS $=1111$

BLOCK FILTER SEP

PARAM PRES $=1$

FRAC STREAM $=$ METALS SUBSTREAM $=$ MIXED COMPS $=\mathrm{C} C-\mathrm{S}$ H2 O2 N2 \& S CL2 F2 NO SO2 H2O SIO2 SIO2-S AL2O3 AL2O3-S FE \& AS BA CD CR PB HG SE AG NAOH NACL $\mathrm{CO} 2 \mathrm{CO} 03 \mathrm{HCL} \&$ FECL3 $\mathrm{H} 2 \mathrm{SO} 4 \mathrm{NA} 2 \mathrm{SO} 4 \mathrm{CH} 4$ POLYMER CEMENT CASO4 AGNO3 \& HNO3 HNO2 NO3- H+ E- AG+ AG+ + 2202 AGCL CL- CU \& OH-RAD NH3 NANO3 FRACS $=000000000001 \&$

$111111111111000000101 \&$

0111000000000101001

FRAC STREAM=METALS SUBSTREAM=CISOLD COMPS=C-S SIO2-S \& 


\section{AL2O3-S CEMENT FRACS $=1111$}

BLOCK MEO-SPLT SEP

DESCRIPTION "for the moment, assumes complete separation"

FRAC STREAM $=$ MEO-LIQ SUBSTREAM $=$ MIXED COMPS=C C-S H2 O2 \& N2 S CL2 F2 NO NO2 H2O SIO2 SIO2-S AL2O3 AL2O3-S \& FE AS BA CD CR PB HG SE AG NAOH NACL CO2 $\mathrm{CO} O 3$ \& HCL FECL3 H2SO4 NA2SO4 CH4 POLYMER CEMENT CASO4 AGNO3 \& HNO3 $\mathrm{HNO} 2 \mathrm{NO} 3-\mathrm{H}+\mathrm{E}-\mathrm{AG}+\mathrm{AG}++\mathrm{H} 2 \mathrm{O} 2 \mathrm{AGCL}$ FRACS $=00$ \& $100010001000000000000 \&$ $000000001000000000100 \&$ 000

FRAC STREAM=MEO-LIQ SUBSTREAM $=$ CISOLD COMPS $=$ C-S SIO2-S \& AL2O3-S CEMENT FRACS $=0000$

FRAC STREAM $=$ HNO2 SUBSTREAM $=$ MIXED COMPS $=\mathrm{C} C-\mathrm{S}$ H2 $\mathrm{O} 2 \mathrm{~N} 2$ \& $S$ CL2 F2 NO H2O SIO2 SIO2-S AL2O3 AL2O3-S FE AS BA \& CD CR PB HG SE AG NAOH NACL CO2 CO O3 HCL FECL3 \& H2SO4 NA2SO4 CH4 POLYMER CEMENT CASO4 AGNO3 HNO3 HNO2 \& NO3- $\mathrm{H}+\mathrm{E}-\mathrm{AG}+\mathrm{AG}+\mathrm{H} 2 \mathrm{O} 2 \mathrm{AGCL}$ FRACS $=000100$ \& $000000000000000000010 \&$ 0000010000010000000

FRAC STREAM $=$ HNO2 SUBSTREAM $=$ CISOLID COMPS $=\mathrm{C}-\mathrm{S}$ SIO2-S \& AL2O3-S CEMENT FRACS $=0000$

FRAC STREAM $=$ NITRIC-2 SUBSTREAM $=$ MIXED $C O M P S=C$ C-S H2 O2 \& N2 S CL2 F2 NO H2O SIO2 SIO2-S AL2O3 AL2O3-S FE AS \& BA CD CR PB HG SE AG NAOH NACL $\mathrm{CO} 2 \mathrm{CO} 03 \mathrm{HCL} \&$ FECL3 $\mathrm{H} 2 \mathrm{SO} 4 \mathrm{NA} 2 \mathrm{SO} 4 \mathrm{CH} 4 \mathrm{POLYMER}$ CEMENT CASO4 AGNO3 \& HNO3 HNO2 NO3- $\mathrm{H}+\mathrm{E}-\mathrm{AG}+\mathrm{AG}++\mathrm{H} 2 \mathrm{O} 2$ AGCL CL- FRACS $=\&$ $000000000000000000000 \&$ 00000000000000010.9999900 \& 0111010

FRAC STREAM=NITRIC-2 SUBSTREAM=CISOLID COMPS=C-S SIO2-S \& AL2O3-S CEMENT FRACS $=0000$

FRAC STREAM $=A C D$-OUT SUBSTREAM $=M$ IXED $C O M P S=S$ H2O FE BA \& CD CR HNO3 FRACS $=1011110.00001$

FRAC STREAM $=A C D$-OUT SUBSTREAM=CISOLD COMPS=C-S SIO2-S \& AL2O3-S CEMENT FRACS $=1111$

\section{BLOCK MET-DCON SEP}

DESCRIPTION "Separates water from metal"

IFRAC STREAM=METLRECY SUBSTREAM=MIXED COMPS=C C-S H2 O2 \& N2 S CL2 F2 NO H2O SIO2 SIO2-S AL2O3 AL2O3-S FE AS \& BA CD CR PB HG SE AG NAOH NACL CO2 CO HCL FECL3 \& $\mathrm{H} 2 \mathrm{SO} 4 \mathrm{NA} 2 \mathrm{SO} 4 \mathrm{CH} 4$ POL YMER CEMENT FRACS $=001000$ \& $000100000000000000000 \&$ 0000000

FRAC STREAM=METLRECY SUBSTREAM=CISOLID COMPS=C-S SIO2-S \& AL2O3-S CEMENT FRACS $=1110$

BLOCK NOX-SPLT SEP

FRAC STREAM=NTTRIC-1 SUBSTREAM=MIXED COMPS $=C$ C-S H2 O2 \& N2 S CL2 F2 NO NO2 $\mathrm{H} 2 \mathrm{O}$ SIO2 SIO2-S AL2O3 AL2O3-S \& FE AS BA CD CR PB HG SE AG NAOH NACL CO2 $\mathrm{CO} O 3 \&$ $\mathrm{HCL}$ FECL3 $\mathrm{H} 2 \mathrm{SO} 4 \mathrm{NA} 2 \mathrm{SO} 4 \mathrm{CH} 4 \mathrm{POLYMER}$ CEMENT CASO4 AGNO3 \& HNO3 HNO2 NO3- H+ E-AG + AG ++ H2O2 AGCL CL- CUNH3 \& NANO3 FRACS $=0000000000000000 \&$ $000000000000000000000 \&$ 01000000000000 
FRAC STREAM=NITRIC-1 SUBSTREAM=CISOLID COMPS=C-S SIO2-S \& AL2O3-S CEMENT FRACS $=0000$

BLOCK OILSEP SEP

DESCRIPTION "assumes $99 \%$ separation of organics $(\mathrm{C}, \mathrm{H}, \mathrm{CHx})$ "

FRAC STREAM $=$ PHOTO-IN SUBSTREAM $=$ MIXED COMPS $=C \mathrm{C}-\mathrm{S}$ H2 $\mathrm{O} 2$ \& N2 S CL2 F2 NO SO2 NO2 H2O SIO2 STO2-S AL2O3 \& AL2O3-S FE AS BA BE CD CR PB HG SE AG TA U NAOH \& NACL $\mathrm{CO} 2 \mathrm{CO} O 3$ HCL FECL3 H2SO4 NA2SO4 CH4 POLYMER \& CEMENT CASO4 AGNO3 HNO3 HNO2 NO3- $\mathrm{H}+\mathrm{E}-\mathrm{AG}+\mathrm{AG}++$ \& H2O2 AGCL CL- CU OH-RAD NH3 NANO3 FRACS $=0.010 .01$ \& $0.011111111111111111111 \&$ $1111111111111110.010 .0111 \&$ 111111111111111

FRAC STREAM=PHOTO-IN SUBSTREAM=CISOLID COMPS=C-S SIO2-S \& AL2O3-S CEMENT FRACS $=0.01111$

BLOCK PB-DECON SEP

FRAC STREAM=LEAD-OUT SUBSTREAM $=$ MIXED COMPS=PB FRACS $=1$

FRAC STREAM $=$ LEAD-OUT SUBSTREAM $=$ CISOLID COMPS $=C-S$ SIO2-S \& AL2O3-S CEMENT FRACS $=0000$

BLOCK PB-SPLIT SEP

FRAC STREAM $=$ PBBRICKS SUBSTREAM $=$ MIXED COMPS $=\mathrm{H} 2$ S FRACS $=\&$ 0.810 .81

MASS-FLOW STREAM=PBBRICKS SUBSTREAM=MIXED COMPS=PB FLOWS $=\&$ 21

FRAC STREAM=PBBRICKS SUBSTREAM=CISOLD COMPS=C-S SIO2-S \& AL2O3-S FRACS $=0.810 .810 .81$

For now, assumes $50 \%$ desorption to gas phase of $\mathrm{C}, \mathrm{H2}, \mathrm{Cl}, \mathrm{F} 2$

BLOCK VACSEP-1 SEP

DESCRIPTION "For now, a simple separation (w/o condensers,etc)"

FRAC STREAM=VACGAS-1 SUBSTREAM=MIXED COMPS=C C-S H2 O2 \& N2 S CL2 F2 NO NO2 H2O SIO2 SIO2-S AL2O3 AL2O3-S \& FE AS BA CD CR PB HG SE AG NAOH NACL $\mathrm{CO} 2 \mathrm{CO} O 3$ \& HCL FECL3 H2SO4 NA2SO4 CH4 POL YMER CEMENT CASO4 AGNO3 \& HNO3 HNO2 NO3-H+E-AG+AG++ $\mathrm{H} 2 \mathrm{O} 2$ AGCL CL-CU \& FRACS $=000010001100000000 \&$ $000000001110000000000 \&$ 0000000000

FRAC STREAM=VACGAS-1 SUBSTREAM $=$ CISOLID COMPS=C-S SIO2-S \& AL2O3-S CEMENT FRACS $=0000$

FRAC STREAM=VACSOL-1 SUBSTREAM $=$ MIXED COMPS $=\mathrm{C}$ C-S H2 O2 \& N2 S CL2 F2 NO NO2 SIO2 SIO2-S AL2O3 AL2O3-S FE AS \& BA BE CD CR PB HG SE AG TA U NAOH NACL $\mathrm{CO} 2 \mathrm{CO} \&$ $\mathrm{O} 3 \mathrm{HCL}$ FECL3 $\mathrm{H} 2 \mathrm{SO} 4 \mathrm{NA} 2 \mathrm{SO} 4 \mathrm{CH} 4 \mathrm{POL}$ YMER CEMENT CASO4 \& AGNO3 HNO3 $\mathrm{HNO} 2 \mathrm{NO} 3-\mathrm{H}+\mathrm{E}-\mathrm{AG}+\mathrm{AG}++\mathrm{H} 2 \mathrm{O} 2 \mathrm{AGCL} \mathrm{CL}-$ \& CUNANO3 FRACS $=000.51010 .50 .500111 \&$

$111111110111111000010 \&$

100111000000001011

MASS-FLOW STREAM=VACSOL-1 SUBSTREAM $=\mathrm{MIXED}$ COMPS $=\mathrm{H} 2 \mathrm{O}$ FLOWS $=\&$ 306

FRAC STREAM=VACSOL-1 SUBSTREAM $=$ CISOLD COMPS=C-S SIO2-S \& AL2O3-S CEMENT FRACS $=0.5111$

FRAC STREAM=VACORG-1 SUBSTREAM=MIXED COMPS=C C-S H2 O2 \& N2 S CL2 F2 NO NO2 H2O SIO2 SIO2-S AL2O3 AL2O3-S \& 
FE AS BA CD CR PB HG SE AG NAOH NACL $\mathrm{CO} 2 \mathrm{CO} O 3$ \& HCL FECL3 H2SO4 NA2SO4 CH4 POL YMER CEMENT CASO4 AGNO3 \& HNO3 $\mathrm{HNO} 2 \mathrm{NO} 3-\mathrm{H}+\mathrm{E}-\mathrm{AG}+\mathrm{AG}++\mathrm{H} 2 \mathrm{O} 2 \mathrm{AGCL}$ CL-CU \&

FRACS $=000.50000 .50 .500000000 \&$ $000000000000000001100 \&$ 000000000000

FRAC STREAM=VACORG-1 SUBSTREAM=CISOLD COMPS=C-S SIO2-S \& AL2O3-S CEMENT FRACS $=0.5000$

FRAC STREAM $=$ VAC-HG-1 SUBSTREAM=MIXED COMPS $=\mathrm{C}$ C-S H2 O2 \& N2 S CL2 F2 NO SO2 NO2 H2O SIO2 SIO2-S AL2O3 \& AL2O3-S FE AS BA BE CD CR PB HG SE AG TA U NAOH \& NACL $\mathrm{CO} 2 \mathrm{CO} O 3$ HCL FECL3 H2SO4 NA2SO4 CH4 POLYMER \& CEMENT CASO4 AGNO3 HNO3 HNO2 NO3- H+E-AG+AG++ \& H2O2 AGCL CL- CU OH-RAD NH3 NANO3 FRACS $=00000$ \& $000000000000000000100 \&$ $000000000000000000000 \&$ 000000000

FRAC STREAM=VAC-HG-1 SUBSTREAM=CISOLID COMPS=C-S SIO2-S \& AL2O3-S CEMENT FRACS $=0000$

;For now, assumes $50 \%$ desorption to gas phase of $\mathrm{C}, \mathrm{H} 2, \mathrm{Cl} 2, \mathrm{~F} 2$

\section{BLOCK VACSEP-3 SEP}

DESCRIPTION "For now, a simple separation (w/o condensers, etc)"

FRAC STREAM=VACGAS-3 SUBSTREAM=MTXED COMPS=C C-S H2 O2 \& N2 S CL2 F2 NO SO2 NO2 H2O SIO2 SIO2-S AL2O3 \& AL2O3-S FE AS BA CD CR PB HG SE AG NAOH NACL CO2 \& CO O3 HCL FECL3 H2SO4 NA2SO4 CH4 POLYMER CEMENT \& CASO4 AGNO3 HNO3 HNO2 NO3- $\mathrm{H}+\mathrm{E}-\mathrm{AG}+\mathrm{AG}++\mathrm{H} 2 \mathrm{O} 2 \mathrm{AGCL} \&$ CL- CU OH-RAD NH3 NANO3 FRACS $=0000010001$ \& $000000000000000000111 \&$ $000000000000000000000 \&$ 00

FRAC STREAM=VACGAS-3 SUBSTREAM=CISOLD COMPS=C-S SIO2-S \& AL2O3-S CEMENT FRACS $=0000$

FRAC STREAM=VACSOL-3 SUBSTREAM=MIXED COMPS=C C-S H2 O2 \& N2 S CL2 F2 NO SO2 NO2 SIO2 SIO2-S AL2O3 AL2O3-S \& FE AS BA CD CR PB HG SE AG NAOH NACL CO2 $\mathrm{COO} 3$ \& HCL FECL 3 H2SO4 NA2SO4 CH4 POLYMER CEMENT CASO4 AGNO3 \& HNO3 HNO2 NO3- $\mathrm{H}+\mathrm{E}-\mathrm{AG}+\mathrm{AG}++\mathrm{H} 2 \mathrm{O} 2 \mathrm{AGCL} \mathrm{CL}-\mathrm{CU} \&$ OH-RAD NH3 NANO3 FRACS $=000.51000 .50 .500$ \& $011111111110111100001 \&$ 110111000000000000000

MASS-FLOW STREAM=VACSOL-3 SUBSTREAM $=$ MIXED COMPS=H2O FLOWS $=$ \& 98.55

FRAC STREAM=VACSOL-3 SUBSTREAM=CISOLID COMPS=C-S SIO2-S \& AL2O3-S CEMENT FRACS $=0.5110$

FRAC STREAM=VACORG-3 SUBSTREAM=MIXED COMPS $=\mathrm{C}$ C-S H2 O2 \& N2 S CL2 F2 NO SO2 NO2 H2O SIO2 SIO2-S AL2O3 \& AL2O3-S FE AS BA CD CR PB HG SE AG NAOH NACL CO2 \& CO 03 HCL FECL3 H2SO4 NA2SO4 CH4 POLYMER CEMENT \& CASO4 AGNO3 HNO3 HNO2 NO3- $\mathrm{H}+\mathrm{E}-\mathrm{AG}+\mathrm{AG}+\mathrm{H} 2 \mathrm{O} 2 \mathrm{AGCL} \&$ CL- SU OH-RAD NH3 NANO3 FRACS $=110.50000 .5$ \& $0.50000000000000000000 \&$ $000000010000000000000 \&$ 00000

FRAC STREAM=VACORG-3 SUBSTREAM=CISOLID COMPS=C-S SIO2-S \& AL2O3-S CEMENT FRACS $=0.5000$ 
FRAC STREAM=VAC-HG-3 SUBSTREAM=MIXXD COMPS=C C-S H2 O2 \& N2 S CL2 F2 NO SO2 NO2 $\mathrm{H}_{2} \mathrm{O} \mathrm{SIO}_{2} \mathrm{SIO}_{2}-\mathrm{S} \mathrm{AL} 2 \mathrm{O} 3$ \& AL2O3-S FE AS BA BE CD CR PB HG SE AG TA UNAOH \& NACL CO2 CO O3 HCL FECL3 H2SO4 NA2SO4 CH4 POLYMER \& CEMENT CASO4 AGNO3 HNO3 HNO2 NO3- $\mathrm{H}+\mathrm{E}-\mathrm{AG}+\mathrm{AG}++$ \& H2O2 AGCL CL- CU OH-RAD NH3 NANO3 FRACS $=000000$ \& $000000000000000000100 \&$ $000000000000000000000 \&$ 000000000

FRAC STREAM=VAC-HG-3 SUBSTREAM=CISOLD COMPS=C-S SIO2-S \& AL2O3-S CEMENI FRACS $=0000$

;Perry's Handbook p. 20-33 shows moisture contents of below $1 \%$ from dryers.

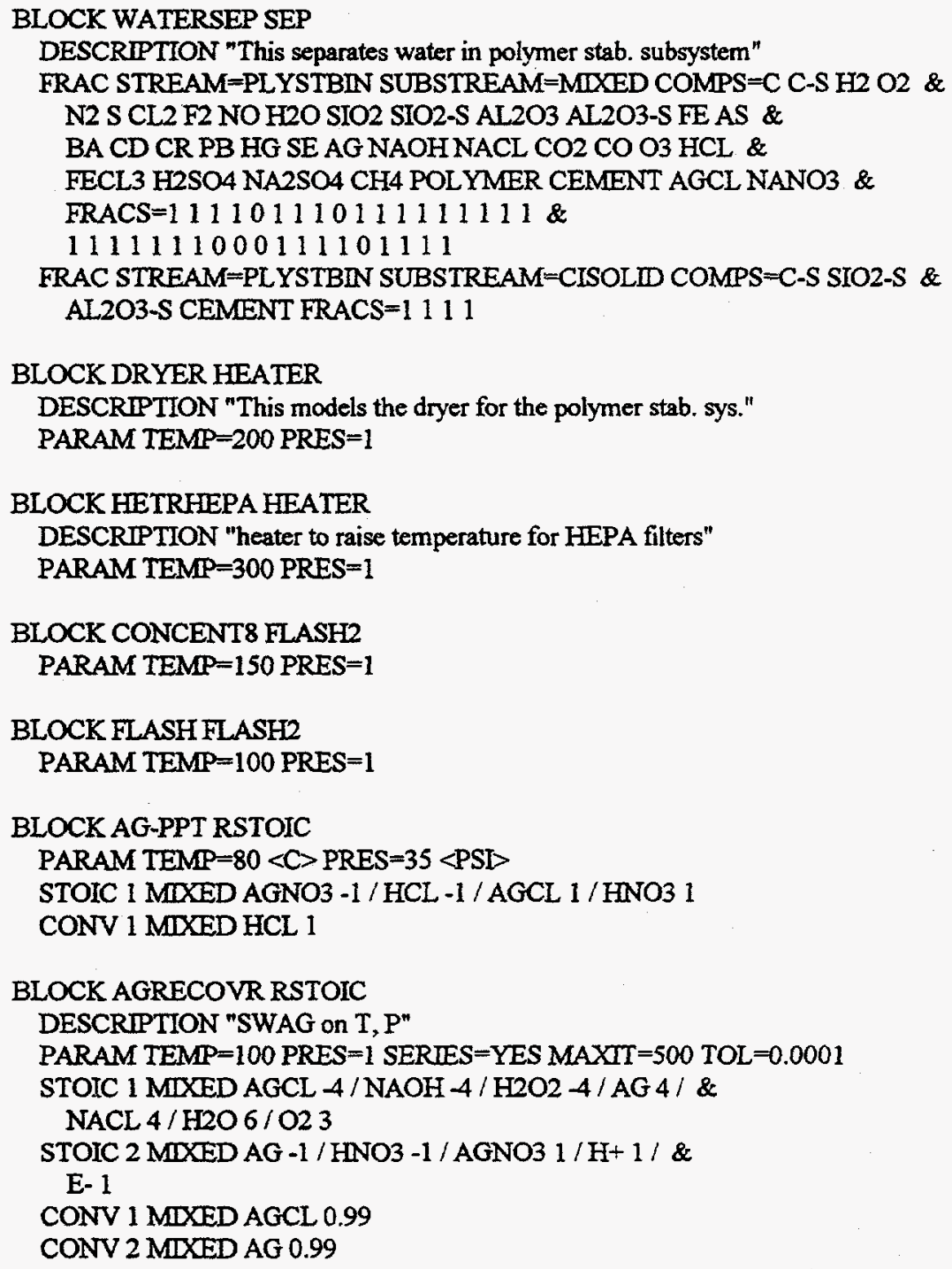

;Data obtained from "Description of Recommended Non-Thermal.."pp. 6-43 to 6-45 ;Although room temperature operation is listed on p. $643,1000 \mathrm{C}$ is listed ;on page $6-45$, and the higher temperature is more believable ;DRE of 0.999 listed for TCE and $0.90-0.95$ for PCE $\rightarrow$ use 0.95 for all waste 


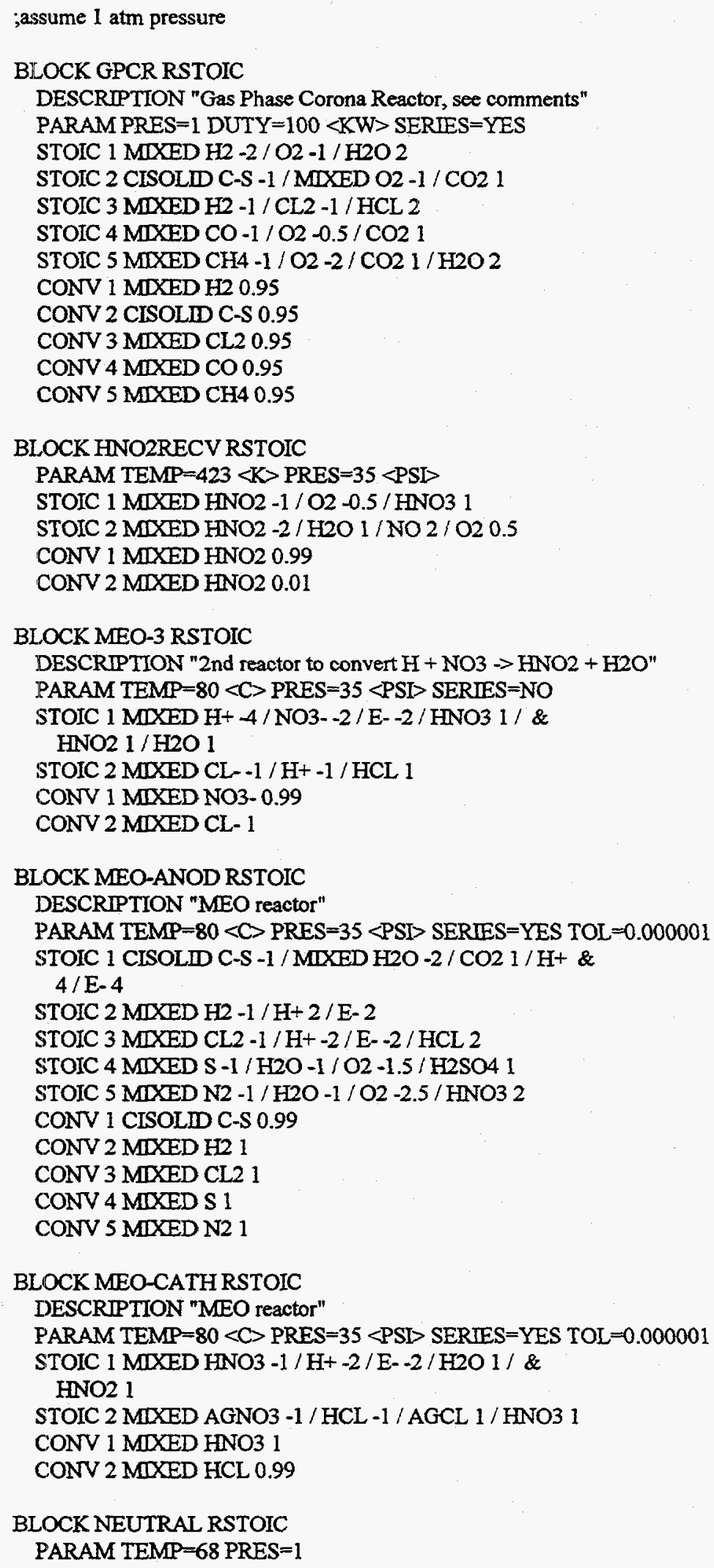


STOIC 1 MIXED NAOH - $1 / \mathrm{HCL}-1 / \mathrm{NACL} 1 / \mathrm{H} 2 \mathrm{O} 1$

STOIC 2 MIXED NAOH - 1 / HNO3 - 1 / NANO3 1 / H2O I

STOIC 3 MIXED CL - - / / NAOH -1 / H + -1/ NACL $1 /$ \& $\mathrm{H} 2 \mathrm{O} 1$

STOIC 4 MIXED NO3- -1/H+ -1/NAOH - $1 /$ NANO3 $1 /$ \& H2O 1

CONV 1 MXXED HCL 0.99

CONV 2 MAXED HNO3 0.99

CONV 3 MIXED CL 1

CONV 4 MAXED NO3- 1

;scrubbing efficiency of 0.99 assumed for now

BLOCK NEUTRAL3 RSTOIC

DESCRIPTION "neutralizes $\mathrm{HCl}$ in offgas stream"

PARAM TEMP=100 PRES $=1$

STOIC 1 MDXED HCL -1 / NAOH - 1 / NACL 1 / H2O 1

STOIC 2 MOXED CL2 - $1 /$ NAOH -2 / NACL 2 / $\mathrm{H}_{2} \mathrm{O} 1 /$ / \& $\mathrm{O} 20.5$

STOIC 3 MIXED H2SO4 - I / NAOH -2 / NA2SO4 1 / H2O 2

STOIC 4 MDXED SO2 - $1 /$ NAOH - 2 / O2 - $0.5 /$ NA2SO4 1 \& $1 \mathrm{H} 2 \mathrm{O} 1$

STOIC 5 MLEED HNO2 -1 / NAOH -1 / O2 - - .5 / H2O 1 / \& NANO3 1

CONV 1 MLXED HCL 0.99

CONV 2 MIXED CL2 0.99

CONV 3 MIXED H2SO4 0.99

CONV 4 MIXED SO2 0.99

CONV 5 MTXED HNO2 1

BLOCK NOX-CONV RSTOIC

PARAM TEMP $=68$ PRES $=1$

STOIC 1 MIXED NO - 6 / NH3 - 4 / N2 5/ H2O 6

CONV 1 MIXED NO 0.99

BLOCK OH-GEN RSTOIC

PARAM TEMP $=100$ PRES $=1$

STOIC 1 MDXED H2O2 - $1 /$ OH-RAD 2

STOIC 2 MIXED O3 -1 / H2O -3 / OH-RAD 6

CONV 1 MDED H2O2 0.99

CONV 2 MIXED O3 0.99

BLOCK OZ-DECOM RSTOIC

DESCRIPTION "ozone decomposer"

PARAM TEMP $=100$ PRES=1

STOIC 1 MIXED $03-2 / 023$

STOIC 2 MIXED HCL - 1 / NAOH - $1 /$ NACL $1 /$ H2O 1

STOIC 3 MIXED SO2 - $1 / \mathrm{NAOH}-2 / \mathrm{O} 2-0.5 / \mathrm{NA} 2 \mathrm{SO} 41$ \& / $\mathrm{H} 2 \mathrm{O} 1$

STOIC 4 MIXED H2SO4 - 1 / NAOH -2/NA2SO4 1 / H2O 2

CONV 1 MIXED 030.999

CONV 2 MIXED HCL 0.99

CONV 3 MXXED SO2 0.99

CONV 4 MIXED H2SO4 0.99

;assumed temperature and pressure

BLOCK OZ-GEN RSTOIC 
DESCRIPTION "generates ozone from air"

PARAM TEMP $=100$ PRES $=1$

STOIC 1 MIXED O2 -3/O3 2

CONV 1 MIXED O2 0.999

;Destruction efficiency of $96 \%$ assumed (p. 2-78 of "Description of Recommended ;Non-Thermal Mixed Waste Treatment Technologies Version 1.0," Emile A. Bernard ;temperature and pressure assumed by BWBrown

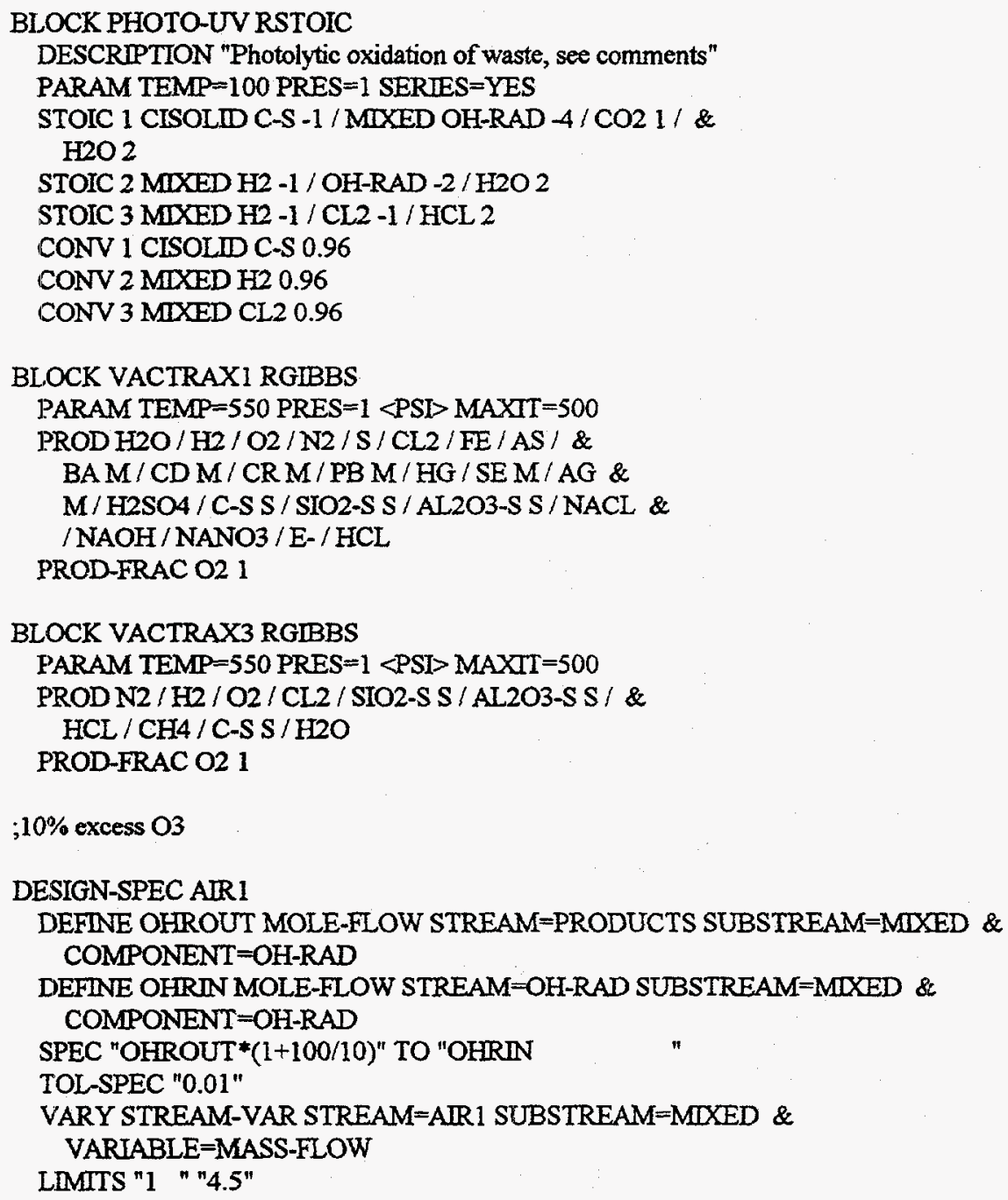

;Ferry's Handbook p. 20.33 has moisture contents about $0.5 \%$ from dryers ;review meeting of $2 / 8 / 96$ : moisture content to $5 \%$

DESIGN-SPEC DRYER

DEFINE H2OMAS MASS-FLOW STREAM=PLYSTBIN SUBSTREAM=MIXED \& COMPONENT $=\mathrm{H} 2 \mathrm{O}$

DEFINE MIXIMAS STREAM-VAR STREAM=PLYSTBIN SUBSTREAM=MTXED \& VARIABLE=MASS-FLOW

DEFINE SOLMAS STREAM-VAR STREAM=PLYSTBIN SUBSTREAM=CISOLID \& VARIABLE=MASS-FLOW

SPEC "H2OMAS" TO "(MIXMAS+SOLMAS)*0.05" 


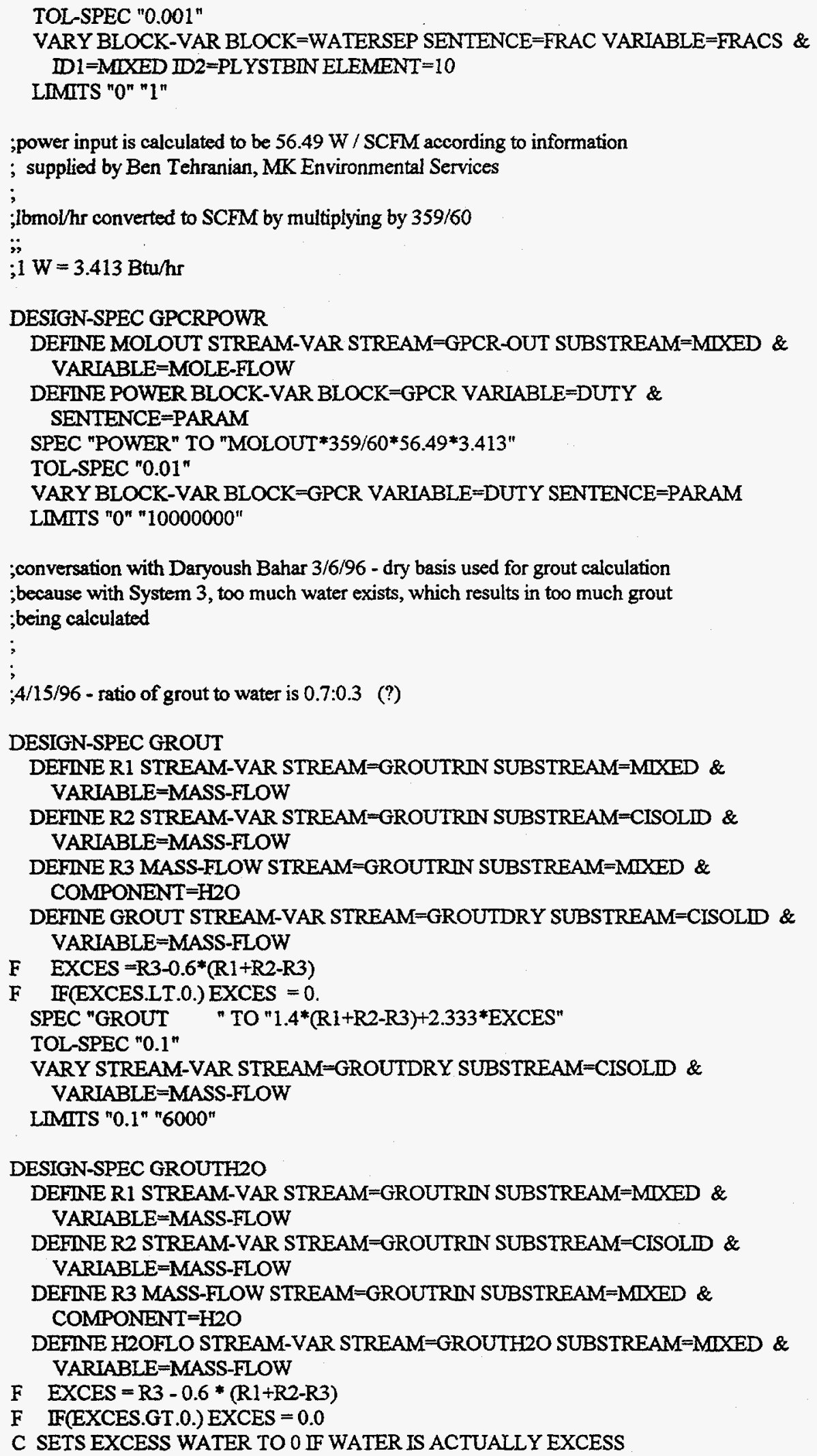


C WATER REQUTRED, IF ANY, IS RETURNED AS A NEGATTVE NUMBER

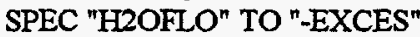

TOL-SPEC "0.01"

VARY STREAM-VAR STREAM=GROUTH2O SUBSTREAM=MIXED \& VARIABLE $=$ MASS-FLOW

LIMITS "0" "2000"

DESIGN-SPEC H2O-IN

DEFINE H2OIN MASS-FLOW STREAM=MEO-N SUBSTREAM=MIXED \& COMPONENT $=\mathrm{H} 2 \mathrm{O}$

DEFINE H2OOUT MASS-FLOW STREAM=MEO1-OUT SUBSTREAM=MDED \& COMPONENT $=\mathrm{H} 2 \mathrm{O}$

SPEC "H2ODN" TO "H2OOUT* $(1+100 / 10)$ "

TOL-SPEC "1.0"

VARY BLOCK-VAR BLOCK=MEO-SPLT SENTENCE=FRAC VARIABLE=FRACS \& $\mathrm{D1} 1=\mathrm{MIXED}$ ID2=MEO-LIQ ELEMENT $=11$

LIMITS "0.330" "0.345"

DESIGN-SPEC HCL-IN

DEFINE HCLOUT MOLE-FLOW STREAM=PPT-OUT SUBSTREAM=MIXED \& COMPONENT $=\mathrm{HCL}$

DEFINE HCLIN MOLE-FLOW STREAM=HCL-IN SUBSTREAM=MIXED \& COMPONENT $=\mathrm{HCL}$

DEFINE CLOUT MOLE-FLOW STREAM=PPT-OUT SUBSTREAM=MIXED \& COMPONENT $=\mathrm{CL}$.

SPEC "HCLIN "TO "HCLOUT* $(1+100 / 1) "$

TOL-SPEC "0.01"

VARY STREAM-VAR STREAM=HCL-IN SUBSTREAM=MIXED \& VARIABLE=MOLE-FLOW

LIMITS "0.00001" "60"

; This design spec is merely to provide enough

; HNO3 to allow the reaction to proceed ( $1 \%$ excess).

DESIGN-SPEC HNO3-IN1

DEFTNE HNO3OT MASS-FLOW STREAM=AGRECOVR SUBSTREAM=MIXED \& COMPONENT $=\mathrm{HNO} 3$

DEFINE NO3IN1 MASS-FLOW STREAM=NITRIC-2 SUBSTREAM=MIXED \& COMPONENT $=$ HNO3

DEFINE NO3IN2 MASS-FLOW STREAM $=$ HNO3-IN1 SUBSTREAM=MTXED \& COMPONENT $=$ HNO3

SPEC "NO3IN1+NO3N2 "TO \& "HNO3OT* $(1+100 / 1)$

TOLSSPEC "0.0001"

VARY STREAM-VAR STREAM=HNO3-IN1 SUBSTREAM=MTXED \& VARIABLE $=$ MASS-FLOW

LIMTTS "0.0001" " $90000 "$

DESIGN-SPEC NAOH-1

DEFINE NAOHIN MASS-FLOW STREAM=NAOH-1 SUBSTREAM=MIXED \& COMPONENT $=\mathrm{NAOH}$

DEFINE NAOHOT MASS-FLOW STREAM=AGRECOVR SUBSTREAM=MIXED \& COMPONENT $=\mathrm{NAOH}$

DEFINE AGCLIN MASS-FLOW STREAM =NITRIC-2 SUBSTREAM=MIXED \& COMPONENT $=$ AGCL

DEFINE AGCLOT MASS-FLOW STREAM=AGRECOVR SUBSTREAM=MIXED \& COMPONENT $=A G C L$

SPEC "NAOHIN" TO "NAOHOT* $(1+100 / 1)$ " 


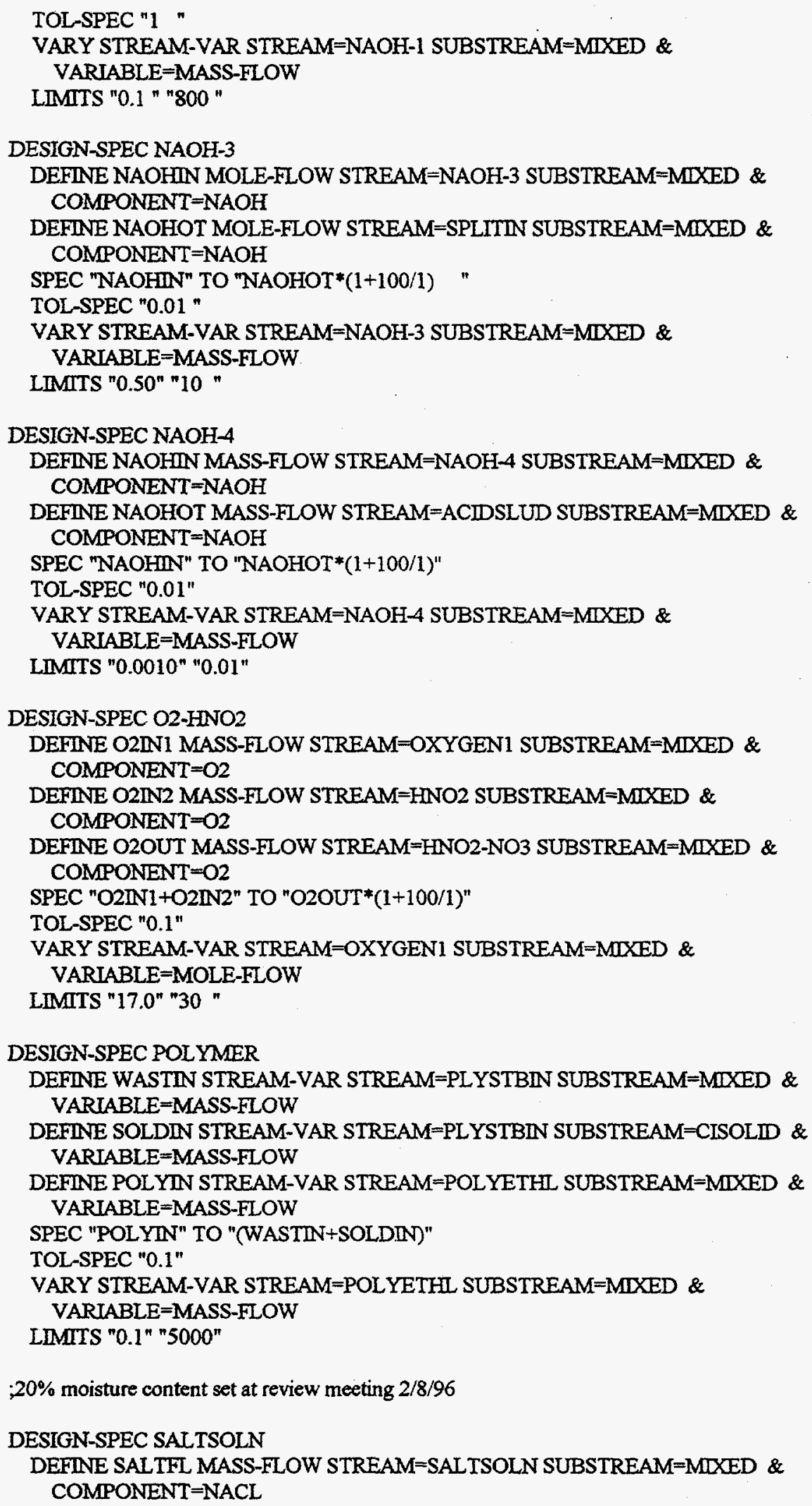




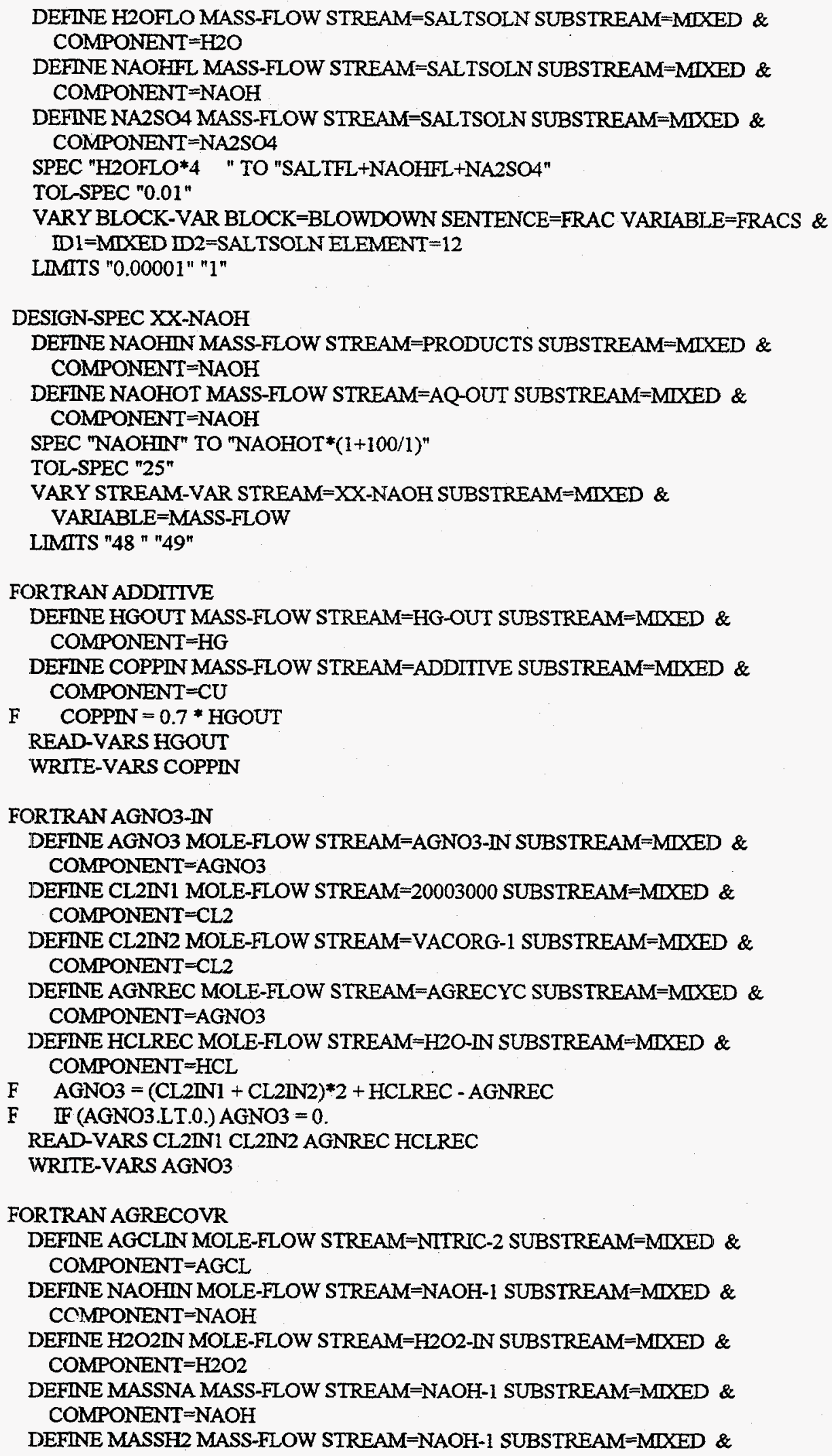




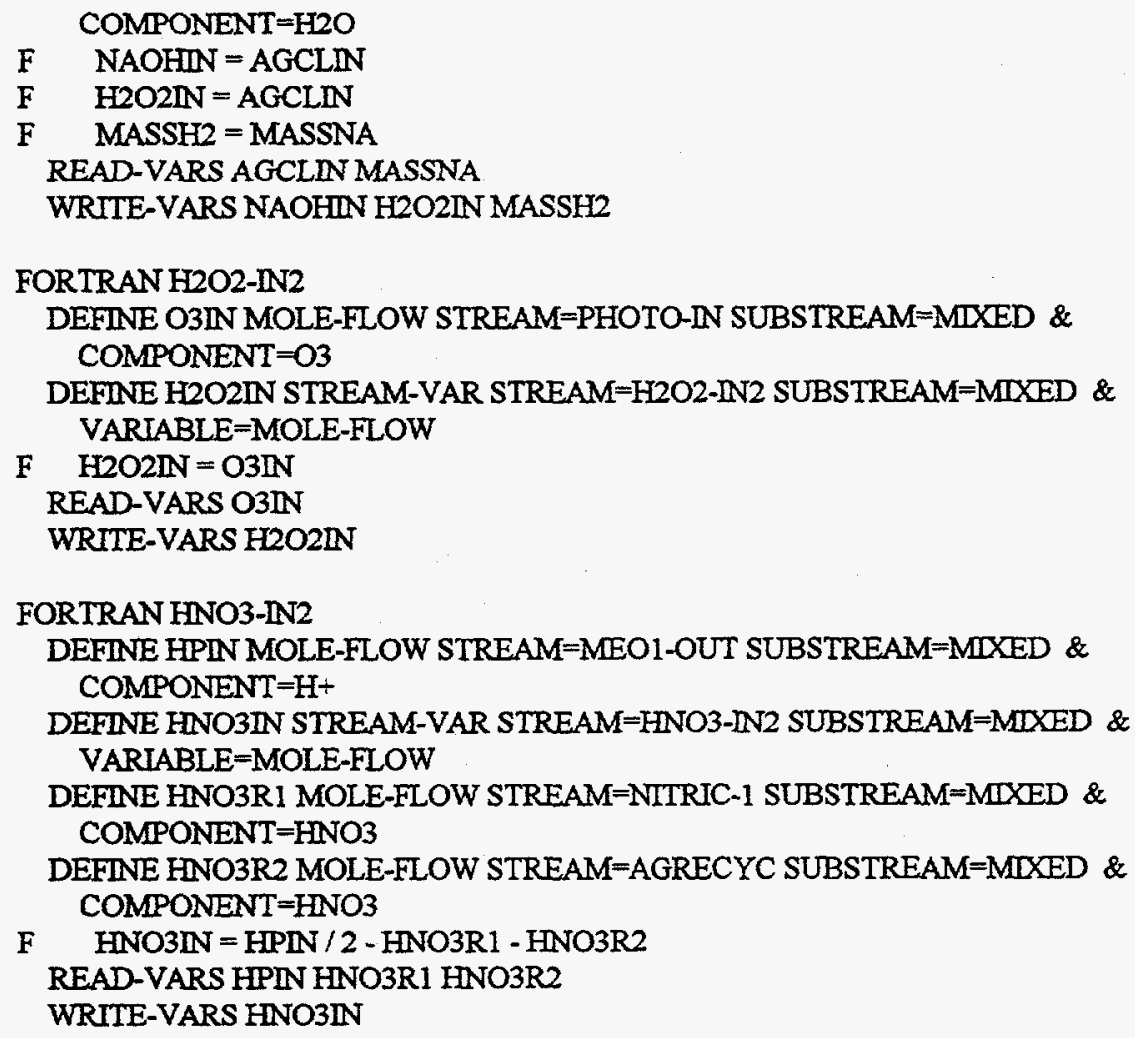


CONV-OPTIONS

PARAM TEAR-NETHOD=BROYDEN TOL $=0.0011$

WEGSTEIN MAXIT $=9999$ ACCELERATE $=0$ NACCELERATE $=1$

STREAM-REPOR MOLEFLOW MASSFLOW

;

;

;

;

B-24 


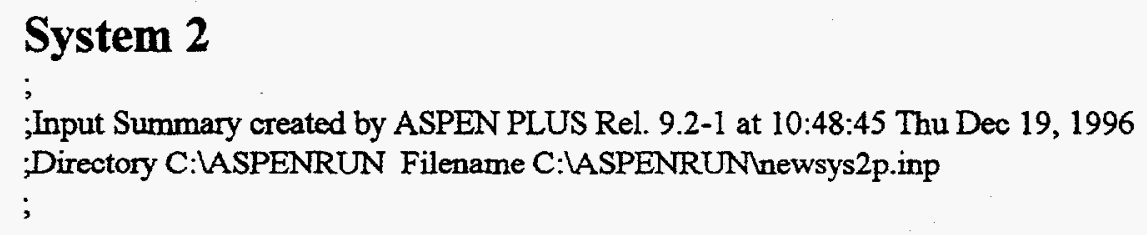

TIILE 'This is System 2 for INTS'

IN-UNITS ENG PRESSURE=ATM

DEF-STREAMS MIXCISLD ALL

DATABANKS COMBUST / INORGANIC / PURECOMP / SOLDS / \& NOASPENPCD

PROP-SOURCES COMBUST / INORGANIC / PURECOMP / SOLIDS

;Cement approximated by $\mathrm{CaA} 12 \mathrm{SiO} 6$ (CaO.A12O3.SiO2), Shreve \& Brink p. 162

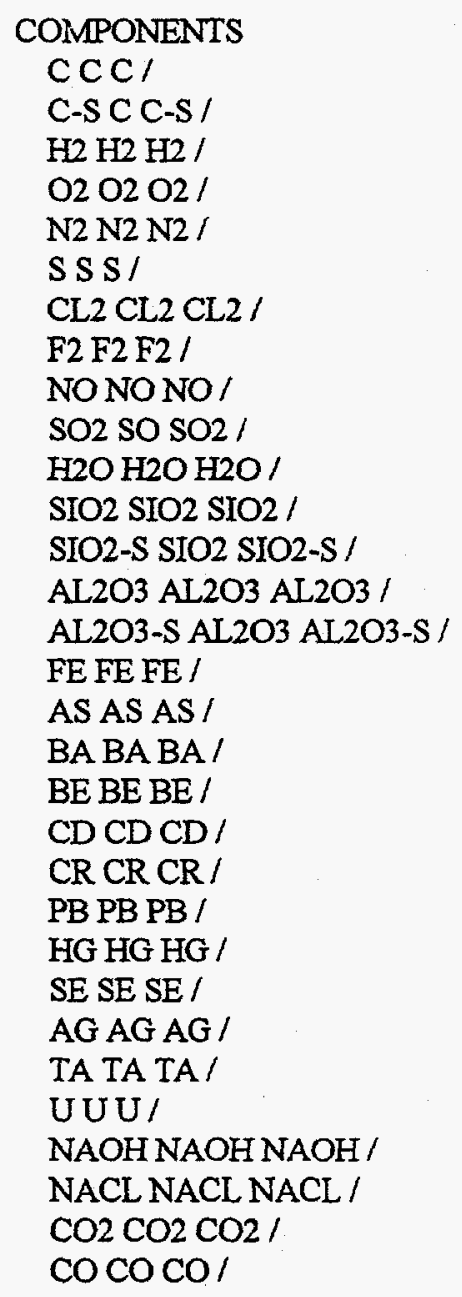


$\mathrm{O} 3 \mathrm{O} 3 \mathrm{O} 3 /$

$\mathrm{H} 2 \mathrm{O} 2 \mathrm{H}_{2} \mathrm{O} 2 \mathrm{H} 2 \mathrm{O} 2 /$

HCL HCL HCL /

FECL3 FECL3 FECL3/

$\mathrm{H}_{2} \mathrm{SO} 4 \mathrm{H} 2 \mathrm{SO} 4 \mathrm{H}_{2} \mathrm{SO}_{4} /$

$\mathrm{NA} 2 \mathrm{SO} 4 \mathrm{NA} 2 \mathrm{SO} 4 \mathrm{NA} 2 \mathrm{SO} 4 /$

$\mathrm{CH} 4 \mathrm{CH} 4 \mathrm{CH} 4$ /

POLYMER C2H4 POLYMER/

CEMENT CAAL2SIO6 CEMENT /

$\mathrm{CASO} 4 \mathrm{CASO} 4 \mathrm{CASO} 4 /$

CUCU CU /

"FE(OH)3" "FE(OH) 3" "FE(OH)3" /

OH-RAD OH OH-RAD

FLOWSHEET APC-SYS

BLOCK GPCR IN=FILTERED AIR4 OUT=GPCR-OUT

BLOCK FILTER IN=AQ-OFGAS EQUIL1 VACGAS-1 VACGAS-2 VACGAS-3 \& OUT=FIL TERED METALS

BLOCK NEUTRAL3 IN=NAOH-3 GPCR-OUT OUT=SPLITIN

BLOCK BLOWDOWN IN=SPLITIN OUT=FLASH-IN SALTSOLN

BLOCK FLASH IN=FLASH-IN OUT=HEATERIN MOISTURE

BLOCK HETRHEPA IN=HEATERIN OUT=OFFGAS

FLOWSHEET AQUEOUS

BLOCK AQ-SPLIT IN=AQ-OUT OUT=AQ-SLUDG AQ-OFGAS AQ-RECYC

BLOCK OZ-GEN IN=AIR 1 OUT $=O Z O N E$

BLOCK CONCENT8 $\mathbb{N}=$ SALTSOLN OUT $=A Q-R E C Y 2$ CONCENT 8

BLOCK PHOTOMIX IN=VACLIQ-2 VACLIQ-1 METLRECY 1000 VACLIQ-3 \& SPLT-H2O H2ORECYC OZONE MOISTURE H2O2-IN2 XX-NAOH \& $A Q-R E C Y 2$ OUT $=X-P H O T N$

BLOCK OH-GEN $\mathbb{N}=$ PHOTO- $\mathbb{N}$ OUT $=O H-R A D$

BLOCK PHOTO-UV IN=OH-RAD OUT=PRODUCTS

BLOCK OZ-DECOM $\mathbb{N}=$ =PRODUCTS OUT=AQ-OUT

BLOCK OILSEP IN=X-PHOTIN OUT=PHOTO-IN X-ORGS

FLOWSHEET BULKSOIL

BLOCK VACSEP-3 $\mathrm{N}=$ =VACOUT-3 XYLEACH 3 OUT=VACLIQ-3 VACGAS-3 \& VACSOL-3 VACORG-3 VAC-HG-3

BLOCK VACTRAX3 $\mathbb{N}=4000$ N2SWEEP 3 OUT=VACOUT -3

FLOWSHEET GROUT

BLOCK GROUTMIX IN=PBGLOVES VACSOL-3 VACSOL-2 OUT=GROUTRIN

BLOCK GROUTER IN=GROUTDRY GROUTRIN GROUTH2O OUT=GROUTOUT

FLOWSHEET HG-AMAL

BLOCK HG-AMALG IN=VAC-HG-2 VAC-HG-3 ADDITIVE 7000-HG \& VAC-HG-1 OUT $=$ HG-OUT

FLOWSHEET METLDCON

BLOCK MET-DCON IN=METLWASH 5000METL OUT=METALOUT METLRECY

FLOWSHEET ORGDEST

BLOCK CWOSPLT IN=CWO-OUT SCRBSALT OUT=CWO-LIQ CWO-SLD \& 
CWO-GAS

BLOCK CWO-I IN=ACD-IN 20003000 OXYGEN1 VACORG-2 VACORG-1 \& VACORG-3 X-ORGS OUT $=$ CWO-OUT

BLOCK NEUTRAL 1 IN=NAOH-1 CWO-LIQ OUT=SCRBOUT 1

BLOCK EQUIL-1 IN=CWO-GAS OUT=EQUIL 1

BLOCK SALTFLTR IN=SCRBOUT 1 OUT $=$ SPLT-H2O SCRBSALT

FLOWSHEET PB-RECVR

BLOCK PB-SPLIT IN=7000-PB OUT=PBBRICKS PBGLOVES

BLOCK PB-DECON IN=PBBRICKS PB-WATER OUT=LEAD-OUT PBSLUDGE

FLOWSHEET POLYMER

BLOCK POLYSTAB IN=PLYSTBIN POLYETHL OUT=POL Y-OUT

BLOCK POL Y-MIX IN=3000POLY CWO-SLD AQ-SLUDG CONCENT \& \& METALS VACSOL-1 OUT $=$ MIXED-IN

BLOCK DRYER IN=MIXED-IN OUT=HOT-MIX

BLOCK WATERSEP IN=HOT-MIX OUT=PLYSTBIN H2ORECYC

FLOWSHEET RES-SLUD

BLOCK DEBR-MIX IN $=5000$ COMP 5000SOFT 5000OPEN OUT $=5000$

BLOCK VACSEP- 2 IN=VACOUT -2 XYLEACH 2 OUT=VACLIQ-2 VACGAS- 2 \& VACSOL-2 VACORG-2 VAC-HG-2

BLOCK VACTRAX2 $\mathbb{N}=5000$ N2SWEEP2 OUT=VACOUT -2

BLOCK VACTRAX1 IN=3000 PBSLUDGE N2SWEEP1 OUT=VACOUT-1

BLOCK VACSEP-1 IN=VACOUT- 1 XYLEACHI OUT=VAC-HG- 1 VACSOL-1 \& VACLIQ-1 VACGAS-1 VACORG-1

FLOWSHEET SPECIAL

BLOCK SPESHULL $\mathbb{N}=6000$ OUT $=$ SPEC-OUT

PROPERTIES SOLIDS

PROPERTIES IDEAL

USER-PROPS DRUSR2 123

PROP-DATA U-2

IN-UNTTS ENG PRESSURE=ATM

PROP-LIST TC

PVAL "FE(OH)3" 100

PROP-DATA U-1

IN-UNITS ENG PRESSURE $=A T M$

PROP-LIST PLXANT

PVAL "FE(OH)3" 7.511-1406221000001000

PROP-DATA U-1

N-UNTTS ENG PRESSURE=ATM

PROP-LIST DHVLWT

PVAL "FE(OH)3" 110.3800

;Tables dated 04/24/96 from Daryoush Bahar, MK, (415) 442-7688

;Glass inerts assumed to be $50 \% \mathrm{SiO} 2$ and $50 \% \mathrm{Al} 2 \mathrm{O} 3$ for all waste streams. 


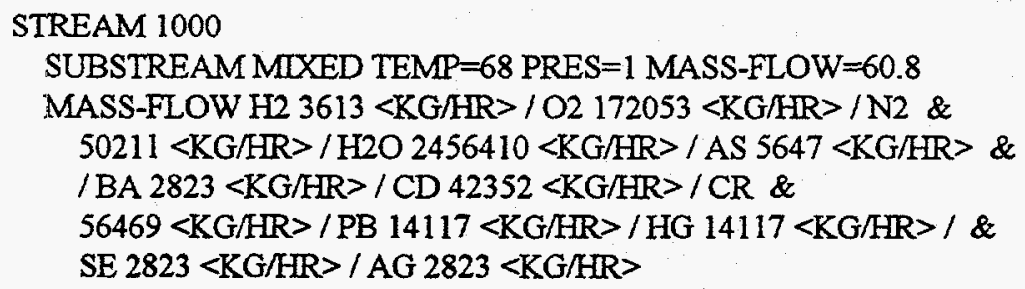

;Tables dated 04/24/96 from MK

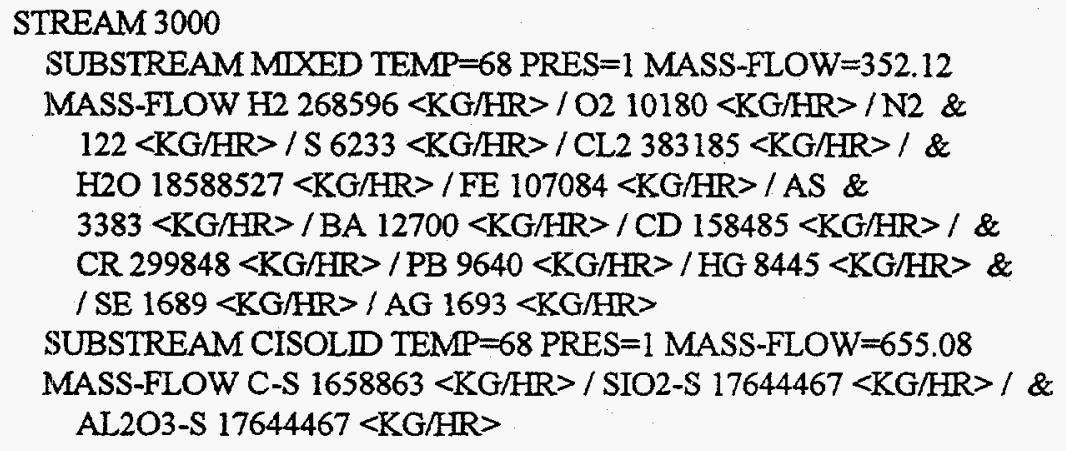

;tables dated 04/24/96 from MK

STREAM 4000

SUBSTREAM MEXED TEMP $=68$ PRES $=1$ MASS-FLOW $=40.74$

MASS-FLOW H2 $21653<\mathrm{KG} / \mathrm{HR}>$ / CL2 $761580<\mathrm{KG} / \mathrm{HR}>/ \mathrm{H} 2 \mathrm{O}$ \& $1514279<\mathrm{KG} / \mathrm{HR}>$

SUBSTREAM CISOLD TEMP $=68$ PRES $=1$ MASS-FLOW $=287.76$

MASS-FLOW C-S $175090<\mathrm{KG} / \mathrm{HR}>$ / SIO2-S $8027287.5<\mathrm{KG} / \mathrm{HR}>$ / \& AL2O3-S 8027287.5 <KG/HR>

;Data table dated 04/24/96. Supplied by Daryoush Bahar, MK (415) 442-7688

\author{
STREAM 5000COMP \\ SUBSTREAM MIXED TEMP $=68$ PRES $=1$ \\ MASS-FLOW H2 3.026/ H2O 0.197/FE 148.890/ HG 1.3 \\ SUBSTREAM CISOLID TEMP $=68$ PRES $=1$ \\ MASS-FLOW C-S 17.992 / SIO2-S 3.003 / AL2O3-S 3.003 \\ ;tables dated 04/24/96 from MK \\ STREAM 5000METL \\ SUBSTREAM MIXED TEMP $=68$ PRES=1 MASS-FLOW $=434.82$
}


MASS-FLOW H2 141789/FE 13383118

SUBSTREAM CISOLD TEMP=68 PRES=1 MASS-FLOW=27.29

MASS-FLOW C-S 844686 / STO2-S 1981 / AL2O3-S 1981

;tables dated 04/24/96 from MK

STREAM 5000OPEN

SUBSTREAM MIXED TEMP $=68$ PRES $=1$ MASS-FLOW $=0.40$

MASS-FLOW H2 $4587<\mathrm{KG} / \mathrm{HR}>/ \mathrm{O} 218201<\mathrm{KG} / \mathrm{HR}>$

SUBSTREAM CISOLID TEMP $=68$ PRES $=1$ MASS-FLOW $=80$

MASS-FLOW C-S $22543<\mathrm{KG} / \mathrm{HR}>$ / SIO2-S $2243854<\mathrm{KG} / \mathrm{HR}>/$ \& AL2O3-S $2243854<\mathrm{KG} / \mathrm{HR}>$

;tables dated 04/30/96, used mass flow $\mathrm{lb} / \mathrm{hr}$ rather than $\mathrm{kg}$ in waste inventory

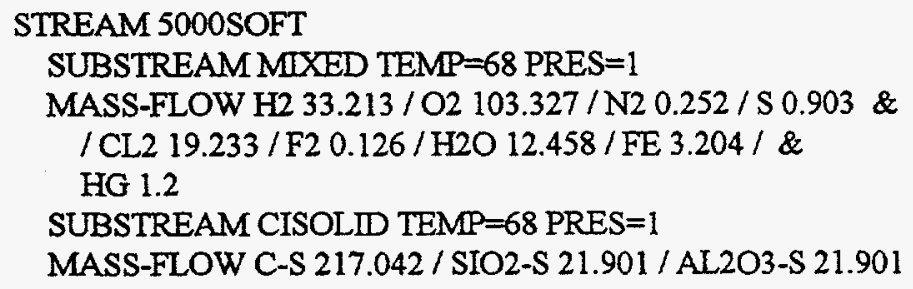

STREAM 5000SOFT

SUBSTREAM MIXED TEMP=68 PRES $=1$

MASS-FLOW H2 33.213/O2 103.327/ N2 0.252/S $0.903 \&$ / CL2 19.233/F2 0.126/H2O 12.458/FE 3.204 / \&

HG 1.2

SUBSTREAM CISOLID TEMP $=68$ PRES $=1$

MASS-FLOW C-S 217.042 / SIO2-S 21.901 / AL2O3-S 21.901

;tables dated 04/24/96 from MK

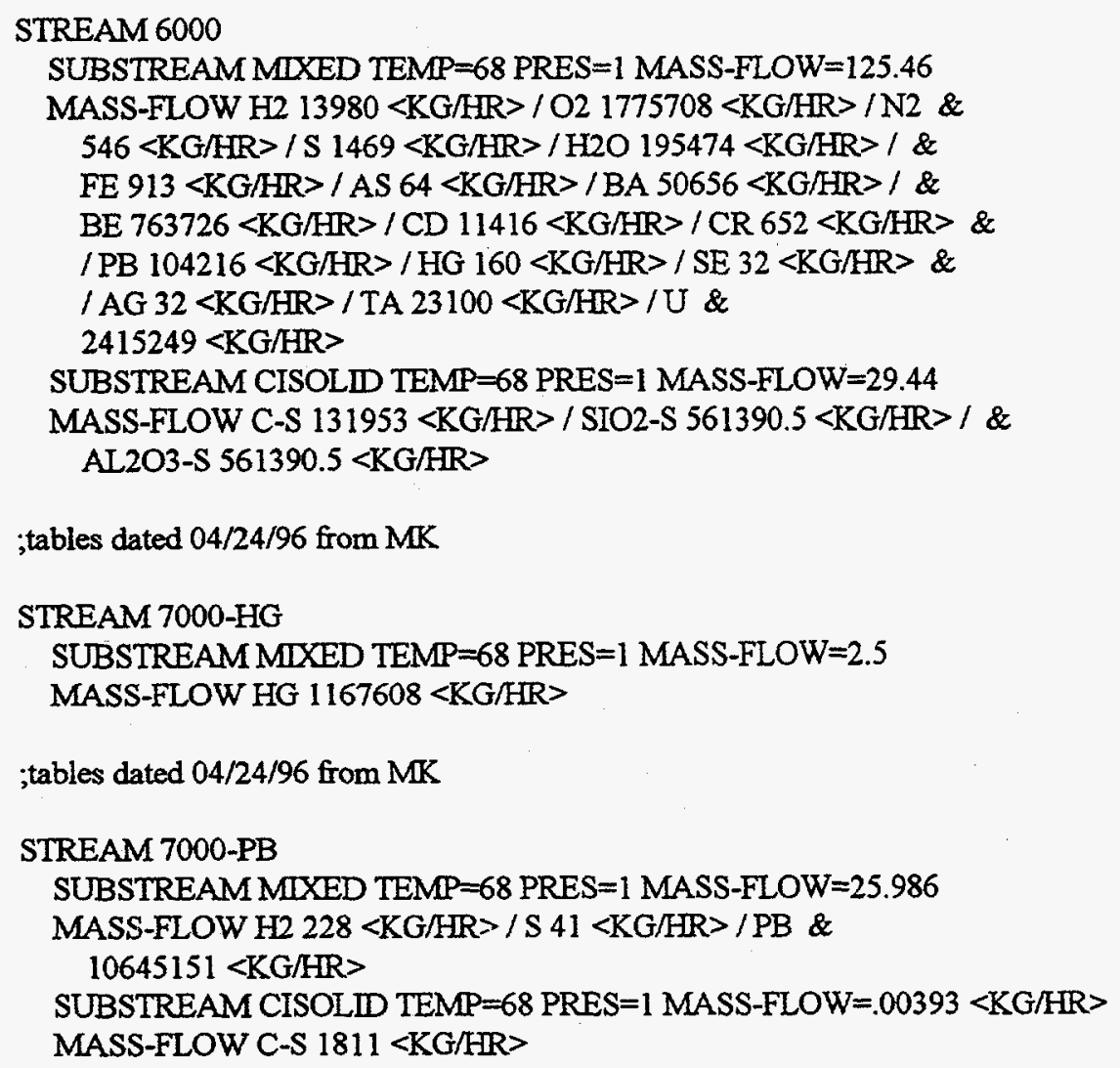


;tables dated 04/24/96 from MK

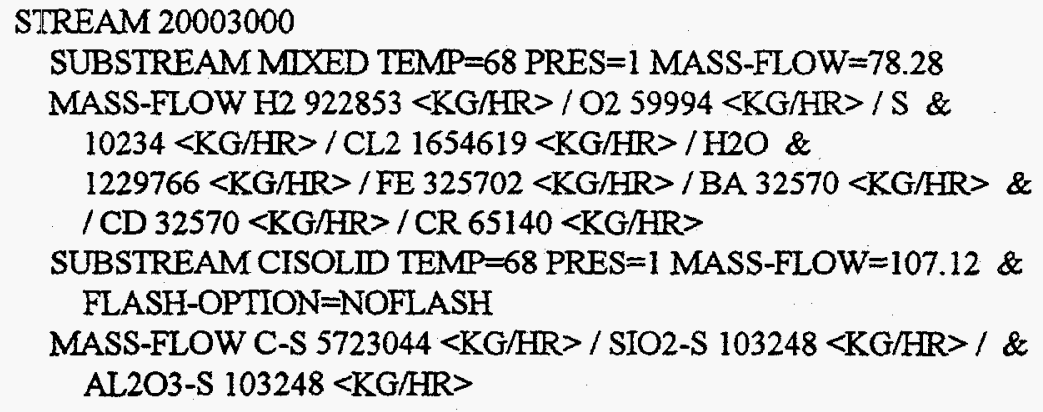

;Water set to 112/468 times the bulk metal flow rate, from Figure 3-22 ; $\quad$ (page 3-46) in ITTS Phase 2 Final Report

STKEAM METLWASH 
SUBSTREAM MIXED TEMP $=68$ PRES $=1$ MASS-FLOW $=94.327$

MASS-FRAC H2O 1

STREAM N2SWEEP1

SUBSTREAM MIXED TEMP $=68$ PRES $=1$ VOLUME-FLOW $=1200$

MOLE-FRAC N2 1

STREAM N2SWEEP2

SUBSTREAM MIXED TEMP $=68$ PRES $=1$ VOLUME-FLOW $=785$

MOLE-FRAC N2 1

STREAM N2SWEEP3

SUBSTREAM MIXED TEMP=68 PRES=1 VOLUME-FLOW=392

MOLE-FRAC N2 1

;According to Delphi flowsheet D-1002A, $50 \% \mathrm{NaOH}$ solution

STREAMNAOH-1

SUBSTREAM MIXED TEMP=68 PRES=1 MASS-FLOW=1

MASS-FRAC $\mathrm{H} 2 \mathrm{O} 0.5 / \mathrm{NAOH} 0.5$

STREAMNAOH-3

SUBSTREAM MIXED TEMP=68 PRES=1 MASS-FLOW=1

MASS-FRAC H2O 0.5 / NAOH 0.5

STREAM OXYGEN1

SUBSTREAM MIXED TEMP=68 PRES=1 MASS-FLOW=1

MOLE-FLOW O2 1

STREAM PB-WATER

SUBSTREAM MIXED TEMP $=68$ PRES $=1$ MASS-FLOW $=13$

MASS-FRAC $\mathrm{H} 2 \mathrm{O} 1$

;Polymer assumed to be polyethylene; $\mathrm{C} 2 \mathrm{H} 4$ used as the monomer.

STREAM POLYETHL

SUBSTREAM MIXED TEMP $=68$ PRES $=1$ MASS $-F L O W=1$

MASS-FRAC POLYMER I

STREAM PRODUCTS

SUBSTREAM MIXED TEMP $=100$ PRES $=1$

MASS-FLOW H2 .688 / O2 4.908 / N2 108.8 / NO 2.738/ \& $\mathrm{H} 2 \mathrm{O} 851.703 / \mathrm{AS} 0.16 / \mathrm{BA} 0.08 / \mathrm{CD} 1.2 / \mathrm{CR} \&$

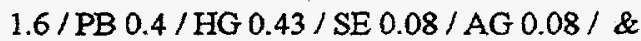

NAOH $0.187 / \mathrm{NACL} 20.7 / \mathrm{O} 32.576 / \mathrm{HCL} 0.13 / \&$

FECL3 0.6

STREAM XX-NAOH

SUBSTREAM MIXED TEMP $=68$ PRES $=1$ MASS-FLOW=1

MASS-FRAC H2O 0.5 / NAOH 0.5

STREAM XYLEACH1

SUBSTREAM MIXED TEMP=68 PRES $=1$ MASS-FLOW=306

B-31 


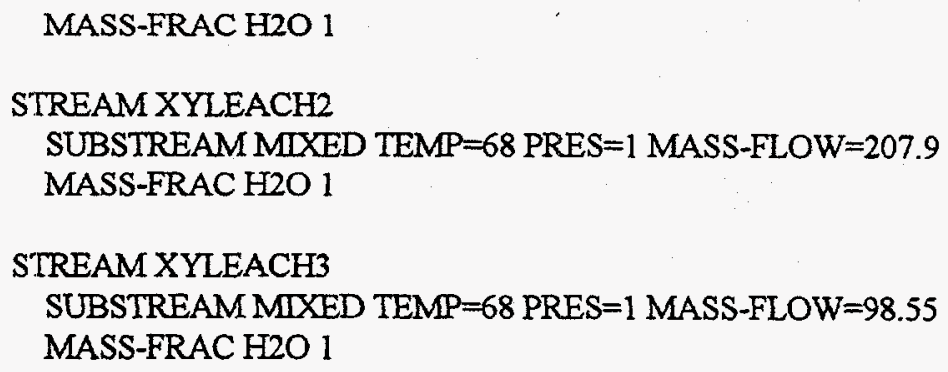

FRAC STREAM=AQ-SLUDG SUBSTREAM=CISOLID COMPS=C-S SIO2-S \& AL2O3-S CEMENT FRACS $=0.990 .990 .990 .99$

FRAC STREAM=AQ-OFGAS SUBSTREAM=MIXED COMPS=C C-S H2 O2 \& N2 S CL2 F2 NO SO2 H2O SIO2 SIO2-S AL2O3 AL2O3-S \& FE AS BA CD CR PB HG SE AG NAOH NACL $\mathrm{CO} 2 \mathrm{CO} O 3 \&$ HCL FECL3 H2SO4 NA2SO4 CH4 POLYMER CEMENT CASO4 \& FRACS $=001110111000000000 \&$ 0000000011100001000

FRAC STREAM=AQ-OFGAS SUBSTREAM=CISOLID COMPS=C-S SIO2-S \& AL2O3-S CEMENT FRACS $=0000$

BLOCK BLOWDOWN SEP

FRAC STREAM=FLASH-IN SUBSTREAM=MIXED COMPS=C C-S H2 O2 \& N2 S CL2 F2 NO SO2 H2O SIO2 SIO2-S AL2O3 AL2O3-S \& 
FE AS BA CD CR PB HG SE AG NAOH NACL CO2 CO O3 \& HCL FECL3 $\mathrm{H} 2 \mathrm{SO} 4$ NA2SO4 CH4 POLYMER CEMENT CASO4 \& FRACS $=11111111110.91111111 \&$ 1111110011111101001

FRAC STREAM=FLASH-IN SUBSTREAM=CISOLID COMPS=C-S SIO2-S \& AL2O3-S CEMENT FRACS $=0000$

BLOCK CWOSPLT SEP

DESCRIPTION "for the moment, assumes complete separation"

FRAC STREAM $=$ CWO-LIQ SUBSTREAM $=$ MIXED COMPS $=\mathrm{C}-\mathrm{S}$ H2 O2 N2 \& $S$ CL2 F2 NO H2O SIO2-S AL2O3-S FE AS BA CD CR PB \& HG SE AG NAOH NACL CO2 HCL FECL3 H2SO4 FRACS $=00$ \& $000000100000000000100 \&$

111

FRAC STREAM $=$ CWO-LIQ SUBSTREAM $=$ CISOLID COMPS=C-S SIO2-S \& AL2O3-S CEMENT FRACS $=0000$

FRAC STREAM $=$ CWO-SLD SUBSTREAM $=$ MIXED COMPS $=\mathrm{C} \mathrm{C}-\mathrm{S} \mathrm{H} 2 \mathrm{O} 2$ \& N2 S CL2 F2 NO H2O SIO2 SIO2-S AL2O3 AL2O3-S FE AS \& BA CD CR PB HG SE AG NAOH NACL CO2 HCL FECL3 \& CEMENT CASO4 CU "FE(OH)3" FRACS $=11000100$ \& 001111111111111010001 \& 111

FRAC STREAM $=$ CWO-SLD SUBSTREAM $=$ CISOLD COMPS=C-S SIO2-S \& AL.2O3-S FRACS $=111$

\section{BLOCK FILTER SEP}

PARAM PRES $=1$

FRAC STREAM $=$ METALS SUBSTREAM $=$ MIXED COMPS $=\mathrm{C}$ C-S H2 O2 N2 \& $\mathrm{S} \mathrm{CL} 2 \mathrm{~F} 2 \mathrm{NO} \mathrm{SO} 2 \mathrm{H} 2 \mathrm{O} \mathrm{SIO} 2 \mathrm{SIO} 2-\mathrm{S} \mathrm{AL} 2 \mathrm{O} 3$ AL2O3-S FE \& AS BA CD CR PB HG SE AG NAOH NACL CO2 CO O3 HCL \& FECL3 H2SO4 NA2SO4 CH4 POLYMER CEMENT CASO4 FRACS $=0$ \& 000000000011111111111 \& 111100001010111

FRAC STREAM=METALS SUBSTREAM=CISOLID COMPS=C-S SIO2-S \& AL2O3-S CEMENT FRACS $=11111$

\section{BLOCK MET-DCON SEP}

DESCRIPTION "Separates water from metal"

FRAC STREAM=METLRECY SUBSTREAM=MIXED COMPS=C C-S H2 O2 \& N2 S CL2 F2 NO H2O SIO2 SIO2-S AL2O3 AL2O3-S FE AS \& BA CD CR PB HG SE AG NAOH NACL CO2 CO HCL FECL3 \& $\mathrm{H} 2 \mathrm{SO} 4 \mathrm{NA} 2 \mathrm{SO} 4 \mathrm{CH} 4$ POLYMER CEMENT FRACS $=0010000$ \& $000100000000000000000 \&$ 0000000

FRAC STREAM=METLRECY SUBSTREAM=CISOLID COMPS=C-S SIO2-S \& AL2O3-S CEMENT FRACS $=1110$

BLOCK OILSEP SEP

DESCRIPTION "assumes $99 \%$ separation of organics (C,H,CHx)"

FRAC STREAM=PHOTO-IN SUBSTREAM=MIXED COMPS=C C-S H2 O2 \& N2 S CL2 F2 NO SO2 H2O SIO2 SIO2-S AL2O3 AL2O3-S \& FE AS BA BE CD CR PB HG SE AG TA UNAOHNACL \& $\mathrm{CO} 2 \mathrm{CO} \mathrm{O} 3 \mathrm{H} 2 \mathrm{O} 2 \mathrm{HCL}$ FECL3 $\mathrm{H} 2 \mathrm{SO} 4 \mathrm{NA} 2 \mathrm{SO} 4 \mathrm{CH} 4 \mathrm{POL}$ YMER \& 
CEMENT CASO4 CU "FE(OH)3" OH-RAD FRACS $=0.010 .010 .01 \&$

111111111111111111111 \&

$11111111111110.010 .011111 \&$

1

FRAC STREAM=PHOTO-IN SUBSTREAM=CISOLID COMPS=C-S SIO2-S \& AL2O3-S CEMENT FRACS $=0.01111$

BLOCK PB-DECON SEP

FRAC STREAM=LEAD-OUT SUBSTREAM=MIXED COMPS=H2O PB FRACS $=$ \& 01

IFAC STREAM=LEAD-OUT SUBSTREAM=CISOLID COMPS=C-S SIO2-S \& AL2O3-S CEMENT FRACS $=0000$

BLOCK PB-SPLIT SEP

FRAC STREAM=PBBRICKS SUBSTREAM $=$ MIXED COMPS=H2 $S$ FRACS $=\&$ 0.810 .81

MASS-FLOW STREAM=PBBRICKS SUBSTREAM=MIXED COMPS=PB FLOWS= \& 21

FRAC STREAM=PBBRICKS SUBSTREAM=CISOLID COMPS=C-S SIO2-S \& AL2O3-S FRACS $=0.810 .810 .81$

BLOCK SALTFLTR SEP

FRAC STREAM $=$ SPLT-H2O SUBSTREAM $=$ MIXED COMPS=C C-S H2 O2 \& N2 S CL2 F2 NO SO2 $\mathrm{H} 2 \mathrm{O}$ SIO2 $\mathrm{SIO} 2-\mathrm{S} \mathrm{AL} 2 \mathrm{O} 3 \mathrm{AL} 2 \mathrm{O} 3-\mathrm{S}$ \& FE AS BA CD CR PB HG SE AG NAOH NACL CO2 CO O3 \& $\mathrm{H} 2 \mathrm{O} 2 \mathrm{HCL}$ FECL3 $\mathrm{H} 2 \mathrm{SO} 4 \mathrm{NA} 2 \mathrm{SO} 4 \mathrm{CH} 4 \mathrm{POLYMER} \mathrm{CEMENT} \mathrm{CASO} 4$ \& CU "FE(OH)3" OH-RAD FRACS=1111111111111 \&

111111111111111111111 \&

111111101

FRAC STREAM=SPLT-H2O SUBSTREAM=CISOLID COMPS=C-S SIO2-S \& AL2O3-S CEMENT FRACS=1 111

;For now, assumes $50 \%$ desorption of $\mathrm{C}, \mathrm{H} 2, \mathrm{Cl} 2, \mathrm{~F} 2$

BLOCK VACSEP-1 SEP

FRAC STREAM=VAC-HG-1 SUBSTREAM=MIXED COMPS=C C-S H2, O2 \& N2 S CL2 F2 NO H2O SIO2 SIO2-S AL2O3 AL2O3-S FE AS \& BA CD CR PB HG SE AG NAOH NACL CO2 CO O3 HCL \& FECL3 H2SO4 NA2SO4 CH4 POLYMER CEMENT CASO4 CU FRACS $=$ \& $000000000000000000001 \&$ 0000000000000000

FRAC STREAM=VAC-HG-1 SUBSTREAM=CISOLID COMPS=C-S SIO2-S \& AL2O3-S CEMENT FRACS $=0000$

FRAC STREAM $=$ VACSOL-1 SUBSTREAM=MIXED COMPS=C C-S H2 O2 \& N2 S CL2 F2 NO SIO2 SIO2-S AL2O3 AL2O3-S FE AS BA \& CD CR PB HG SE AG NAOH NACL CO2 $\mathrm{CO} O 3 \mathrm{HCL}$ FECL3 \& $\mathrm{H} 2 \mathrm{SO} 4 \mathrm{NA} 2 \mathrm{SO} 4 \mathrm{CH} 4 \mathrm{POLYMER}$ CEMENT CASO4 CU FRACS $=11 \&$ $0.51010 .50 .50111111111101 \&$ 111000010100111

MASS-FLOW STREAM=VACSOL-1 SUBSTREAM=MIXED COMPS $=\mathrm{H}-\mathrm{2} 2 \mathrm{O}$ FLOWS $=\&$ 306.

FRAC STREAM=VACSOL-1 SUBSTREAM=CISOLID COMPS=C-S SIO2-S \& AL2O3-S CEMENT FRACS $=0.5111$ 
FRAC STREAM=VACLIQ-1 SUBSTREAM=MIXED COMPS=C C-S H2 O2 \& N2 S CL2 F2 NO SIO2 SIO2-S AL2O3 AL2O3-S FE AS BA \& CD CR PB HG SE AG NAOH NACL CO2 CO O3 HCL FECL 3 \& $\mathrm{H} 2 \mathrm{SO} 4 \mathrm{NA} 2 \mathrm{SO} 4 \mathrm{CH} 4 \mathrm{POLYMER}$ CEMENT CASO 4 CU FRACS $=00$ \& $000000000000000000000 \&$ 0000101000000

MASS-FLOW STREAM=VACLIQ-1 SUBSTREAM=MIXED COMPS $=$ H2O FLOWS $=$ \& 342.5798

FRAC STREAM=VACLIQ-1 SUBSTREAM=CISOLID COMPS=C-S SIO2-S \& AL2O3-S CEMENT FRACS $=0000$

FRAC STREAM=VACGAS-I SUBSTREAM=MIXED COMPS=C C-S H2 O2 \& N2 S CL2 F2 NO H2O SIO2 SIO2-S AL2O3 AL2O3-S FE AS \& BA CD CR PB HG SE AG NAOH NACL CO2 $\mathrm{CO} O 3 \mathrm{HCL} \&$ FECL3 H2SO4 NA2SO4 CH4 POLYMER CEMENT CASO4 CU FRACS $=\&$ 000010001000000000000 \& 0000101000000000

FRAC STREAM=VACGAS-1 SUBSTREAM=CISOLID COMPS=C-S SIO2-S \& AL2O3-S CEMENT FRACS $=0000$

FLASH-SPECS VACLIQ-1 TEMP $=68$ PRES $=1$

FLASH-SPECS VACGAS- 1 TEMP $=68$ PRES $=1$

FLASH-SPECS VAC-HG-1 TEMP $=68$ PRES $=1$

FLASH-SPECS VACSOL- 1 TEMP $=68$ PRES $=1$

FLASH-SPECS VACORG-1 TEMP $=68$ PRES $=1$

;Based on review comments, only $10 \%$ of hydrocarbons will be desorbed from soft

; debris.

BLOCK VACSEP-2 SEP

DESCRIPTION "For now, a simple separation (w/o condensers,etc)"

FRAC STREAM=VACLIQ-2 SUBSTREAM=MIXED COMPS=C C-S H2 O2 \& N2 S CL2 F2 NO SIO2 SIO2-S AL2O3 AL2O3-S FE AS BA \& CD CR PB HG SE AG NAOH NACL CO2 CO O3 HCL FECL3 \& $\mathrm{H} 2 \mathrm{SO} 4 \mathrm{NA} 2 \mathrm{SO} 4 \mathrm{CH} 4$ POLYMER CEMENT CASO4 FRACS $=0000$ \& $000000000000000000000 \&$ 0010000000

MASS-FLOW STREAM=VACLIQ- 2 SUBSTREAM=MIXED COMPS $=\mathrm{H} 2 \mathrm{O}$ FLOWS $=\&$ 12.655

FRAC STREAM=VACLIQ-2 SUBSTREAM=CISOLID COMPS=C-S SIO2-S \& AL 2O3-S CEMENT FRACS $=0000$

FRAC STREAM $=$ VACGAS-2 SUBSTREAM $=$ MIXED $C O M P S=C \mathrm{C}-\mathrm{S}$ H2 $\mathrm{O} 2$ \& N2 S CL2 F2 NO H2O SIO2 SIO2-S AL2O3 AL2O3-S FE AS \& BA CD CR PB HG SE AG NAOH NACL CO2 $\mathrm{CO} O 3 \mathrm{HCL} \&$ FECL3 H2SO4 NA2SO4 CH4 POLYMER CEMENT CASO4 FRACS $=0$ \& $000100010000000000000 \&$ 00011100000000

FRAC STREAM=VACGAS-2 SUBSTREAM=CISOLID COMPS=C-S SIO2-S \& AL2O3-S CEMENT FRACS $=0000$

FRAC STREAM=VACSOL-2 SUBSTREAM=MIXED COMPS=C C-S H2 O2 \& N2 S CL2 F2 NO SIO2 SIO2-S AL2O3 AL2O3-S FE AS BA \& CD CR PB HG SE AG NAOH NACL CO2 CO O3 HCL FECL3 \& H2SO4 NA2SO4 CH4 POLYMER CEMENT CASO4 FRACS $=000.9$ \& $1010.90 .901111111111011 \&$ 1100001110011 
MASS-FLOW STREAM=VACSOL- 2 SUBSTREAM $=$ MIXED COMPS $=$ H2O FLOWS $=\&$ 207.9

FRAC STREAM=VACSOL-2 SUBSTREAM=CISOLD COMPS=C-S SIO2-S \& AL2O3-S CEMENT FRACS $=0.9111$

FRAC STREAM=VACORG-2 SUBSTREAM=MIXED COMPS=C C-S H2 O2 \& N2 S CL2 F2 NO H2O SIO2 SIO2-S AL2O3 AL2O3-S FE AS \&

BA CD CR PB HG SE AG NAOH NACL CO2 $\mathrm{CO} O 3 \mathrm{HCL} \&$ FECL3 H2SO4 NA2SO4 CH4 POLYMER CEMENT CASO4 FRACS $=0$ \& $00.10000 .10 .1000000000000 \&$ 0000000000001100

FRAC STREAM=VACORG-2 SUBSTREAM=CISOLID COMPS=C-S SIO2-S \& AL2O3-S FRACS $=0.100$

FLASH-SPECS VACSOL-2 TEMP=68 PRES $=1$

FLASH-SPECS VACLIQ-2 TEMP $=68$ PRES $=1$

FLASH-SPECS VACGAS-2 TEMP $=68$ PRES $=1$

FLASH-SPECS VACORG- 2 TEMP $=68$ PRES $=1$

FLASH-SPECS VAC-HG-2 TEMP=68 PRES=1

PROPERTIES SOLIDS

BLOCK-OPTION ENERGY-BAL=YES

;For now, assumes $50 \%$ desorption to gas phase of $\mathrm{C}, \mathrm{H} 2, \mathrm{Cl} 2, \mathrm{~F} 2$

BLOCK VACSEP-3 SEP

DESCRIPTION "For now, a simple separation (w/o condensers,etc)"

FRAC STREAM=VACLIQ-3 SUBSTREAM=MIXED COMPS=C C-S H2 O2 \& N2 S CL2 F2 NO SIO2 SIO2-S AL2O3 AL2O3-S FE AS BA \& CD CR PB HG SE AG NAOH NACL CO2 CO O3 HCL FECL3 \& H2SO4 NA2SO4 CH4 POLYMER CEMENT CASO4 FRACS $=0000$ \& $000000000000000000000 \&$ 0010000000

MASS-FLOW STREAM=VACLIQ-3 SUBSTREAM $=$ MIXED $C O M P S=H 2 O$ FLOWS $=$ \& 26.85154

FRAC STREAM=VACLIQ-3 SUBSTREAM=CISOLD COMPS=C-S SIO2-S \& AL2O3-S CEMENT FRACS $=0000$

FRAC STREAM $=$ VACGAS-3 SUBSTREAM=MIXED COMPS $=\mathrm{C}$ C-S H2 O2 \& N2 S CL2 F2 NO H2O SIO2 SIO2-S AL2O3 AL2O3-S FE AS \& BA CD CR PB HG SE AG NAOH NACL CO2 CO O3 HCL \& FECL3 H2SO4 NA2SO4 CH4 POLYMER CEMENT FRACS $=0000$ \& 100010000000000000000 \&

1110000000

FRAC STREAM=VACGAS-3 SUBSTREAM=CISOLID COMPS=C-S SIO2-S \& AL2O3-S CEMENT FRACS $=0000$

FRAC STREAM $=$ VACSOL-3 SUBSTREAM=MIXED COMPS=C C-S H2 O2 \& N2 S CL2 F2 NO SIO2 SIO2-S AL2O3 AL2O3-S FE AS BA \& CD CR PB HG SE AG NAOH NACL CO2 CO O3 HCL FECL 3 \& $\mathrm{H} 2 \mathrm{SO} 4 \mathrm{NA} 2 \mathrm{SO} 4 \mathrm{CH} 4 \mathrm{POLYMER}$ CEMENT CASO4 FRACS $=000.5 \&$ $1000.50 .501111111111011 \&$

1100001110111

MASS-FLOW STREAM=VACSOL-3 SUBSTREAM=MIXED COMPS=H2O FLOWS $=$ \& 98.55

FRAC STREAM=VACSOL-3 SUBSTREAM=CISOLID COMPS=C-S SIO2-S \& AL2O3-S FRACS $=0.511$

FRAC STREAM=VACORG-3 SUBSTREAM=MIXED COMPS=C C-S H2 O2 \& 
N2 S CL2 F2 NO H2O SIO2 SIO2-S AL2O3 AL2O3-S FE AS \& BA CD CR PB HG SE AG NAOH NACL CO2 $\mathrm{CO} O 3 \mathrm{HCL} \&$ FECL3 H2SO4 NA2SO4 CH4 POLYMER CEMENT CASO4 FRACS=1 \& $10.50000 .50 .5000000000000 \&$ 0000000000001000

FRAC STREAM=VACORG-3 SUBSTREAM=CISOLD COMPS=C-S SIO2-S \& AL2O3-S CEMENT FRACS $=0.5000$

;Perry's Handbook p. 20-33 shows moisture contents of below $1 \%$ from dryers.

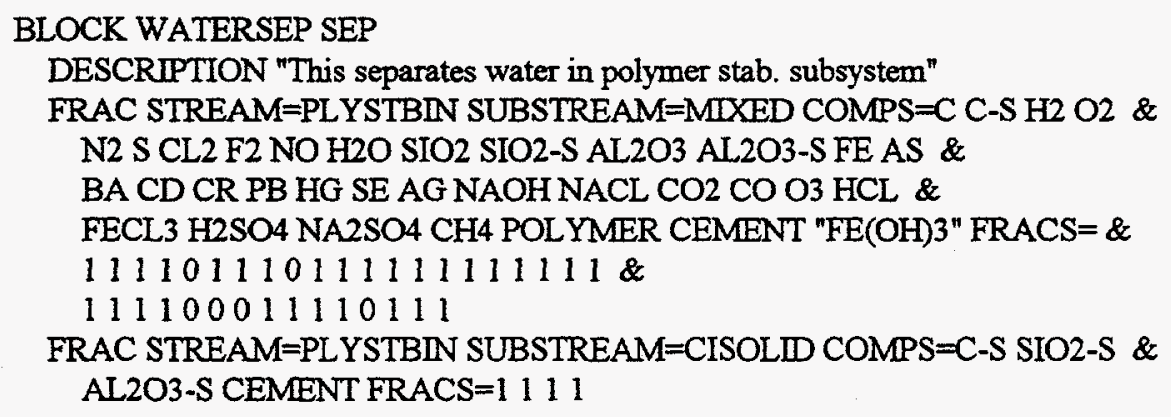

BLOCK DRYER HEATER

DESCRIPTION "This models the dryer for the polymer stab. sys."

PARAM TEMP $=200$ PRES $=1$

BLOCK HETRHEPA HEATER

DESCRIPTION "heater to raise temperature for HEPA filters"

PARAM TEMP $=300$ PRES $=1$

BLOCK CONCENT8 FLASH2

PARAM TEMP $=150$ PRES $=1$

BLOCK FLASH FLASH2

PARAM TEMP $=100$ PRES $=1$

;Data obtained from "Development Studies for a Novel Wet Oxidation Process, ;Topical Report," Patrick M. Dhooge and Lena B. Hakim, Delphi Research, Inc. ;January 1994, DE-AC21-92MC27109

;Operating conditions: page 63 ;Detox solution composition: page 2

;Metal solubility: page xvii, 18

;Reaction stoichiometry: page xiv

;Excess oxygen: page 58

;Extent of reaction: page xvi

$;.$

;Temperature and pressure also reported in "Description of Recommended

;Non-Thermal Mixed Waste Treatment Technologies," p. 2-12

;MK flowsheet shows $423 \mathrm{~K}$ and $20 \mathrm{psi}(+15=35 \mathrm{psia})$

BLOCK CWO-1 RSTOIC

DESCRIPTION "Detox reactor"

PARAM TEMP $=423<\mathrm{K}>$ PRES $=35<$ PSI $>$ SERIES=NO

STOIC 2 CISOLID C-S -1 / MIXED O2 -1/ CO2 1 
STOIC 3 MIXED H2 -1 / O2 $-0.5 / \mathrm{H} 2 \mathrm{O} 1$

STOIC 4 MIXED CL2 - $1 / \mathrm{H} 2-1 / \mathrm{HCL} 2$

STOIC 5 MIXED S -1 / $\mathrm{H} 2$-1/ $/ \mathrm{O} 2$-2 / $\mathrm{H} 2 \mathrm{SO} 41$

STOIC 6 MIXED CH4 -1 / O2 -2 / $\mathrm{CO} 21 / \mathrm{H} 2 \mathrm{O} 2$

STOIC 1 MIXED POL YMER -1 / CISOLID C-S 1 / MIXED CH4 \& 1

CONV 2 CISOLID C-S 0.99

CONV 3 MIXED H2 0.99

CONV 4 MIXED CL2 0.99

CONV 5 MIXED S 0.99

CONV 6 MIXED CH4 0.99

CONV 1 MIXED POLYMER 1

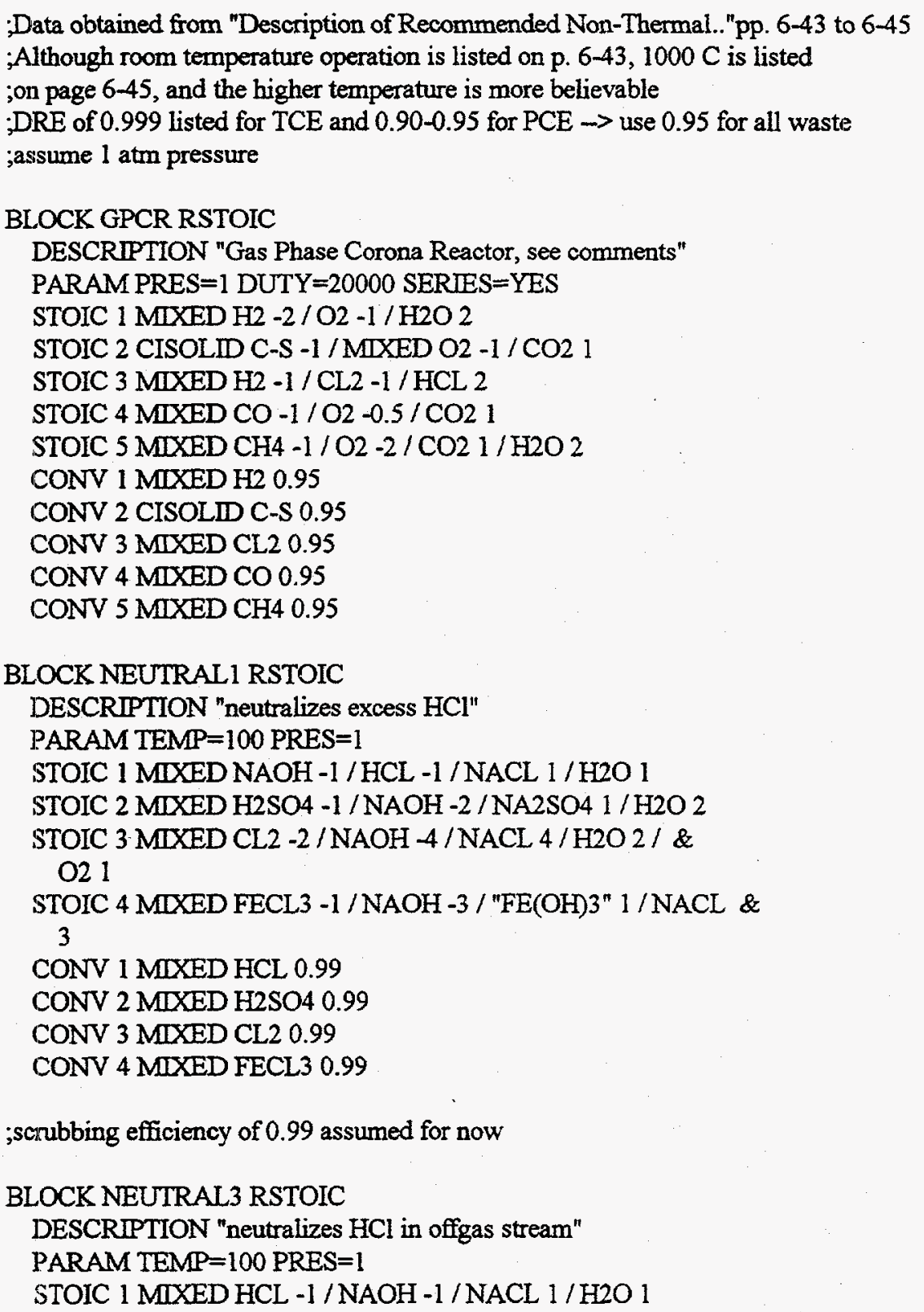


STOIC 2 MIXED CL2 - 1 / NAOH - 2 / NACL $2 / \mathrm{H} 2 \mathrm{O} 1 / \&$ $\mathrm{O} 20.5$

STOIC 3 MIXED H2SO4 - $1 / \mathrm{NAOH}-2 / \mathrm{NA} 2 \mathrm{SO} 41 / \mathrm{H} 2 \mathrm{O} 2$

STOIC 4 MIXED SO2 -1/NAOH - $2 / \mathrm{O} 2-0.5 / \mathrm{NA} 2 \mathrm{SO} 41$ \& $/ \mathrm{H} 2 \mathrm{O} 1$

CONV 1 MIXED HCL 0.99

CONV 2 MIXED CL 20.99

CONV 3 MIXED H2SO4 0.99

CONV 4 MIXED SO2 0.99

BLOCK OH-GEN RSTOIC

PARAM TEMP=100 PRES $=1$

STOIC 1 MIXED H2O2 -1 / OH-RAD 2

STOIC 2 MIXED O3 - 1 / H2O -3 / OH-RAD 6

CONV 1 MIXED H2O2 0.99

CONV 2 MIXED 030.99

BLOCK OZ-DECOM RSTOIC

DESCRIPTION "ozone decomposer"

PARAM TEMP $=100$ PRES $=1$

STOIC 1 MIXED O3 -2/O2 3

STOIC 2 MIXED HCL - $1 /$ NAOH - 1 / NACL $1 /$ H2O 1

STOIC 3 MIXED H2SO4 - $1 / \mathrm{NAOH}$-2/ NA2SO4 $1 /$ H2O 1

STOIC 4 MIXED CL2 - $2 / \mathrm{NAOH}-4 / \mathrm{NACL} 4 / \mathrm{H} 2 \mathrm{O} 2 / \&$

$\mathrm{O} 21$

STOIC 5 MIXED SO2 -2 / NAOH -4 / O2 -1/NA2SO4 2 / \& $\mathrm{H} 2 \mathrm{O} 2$

CONV 1 MIXED O3 0.999

CONV 2 MIXED HCL 0.99

CONV 3 MIXED H2SO4 0.99

CONV 4 MIXED CL2 0.99

CONV 5 MIXED SO2 0.99

;temperature and pressure assumed arbitrarily

BLOCK OZ-GEN RSTOIC

DESCRIPTION "generates ozone from air"

PARAM TEMP $=100$ PRES $=1$

STOIC 1 MIXED O2 -3/O3 2

CONV I MIXED O2 0.999

;Destruction efficiency of $96 \%$ assumed (p. 2-78 of "Description of Recommended ;Non-Thermal Mixed Waste Treatment Technologies Version 1.0," Emile A. Bernard ;temperature and pressure assumed by BWBrown

BLOCK PHOTO-UV RSTOIC

DESCRIPTION "Photolytic oxidation of waste, see comments"

PARAM TEMP=100 PRES=1 SERIES=NO MAXIT $=500$ TOL=0.001 1

STOIC 1 CISOLD C-S -1 / MIXED OH-RAD -4/CO2 $1 / \&$ $\mathrm{H} 2 \mathrm{O} 2$

STOIC 2 MIXED H2 -1 / OH-RAD -2 / $\mathrm{H} 2 \mathrm{O} 2$

STOIC 3 MIXED H2 -1/ CL2 -1/ HCL 2

CONV 1 CISOLID C-S 0.96 
CONV 2 MIXED H2 0.96

CONV 3 MLXED CL2 0.96

BLOCK EQUIL-1 RGIBBS

PARAM TEMP $=423<\mathrm{K}>\mathrm{PRES}=35<\mathrm{PSI}>\mathrm{MAXIT}=500$

;Chuch Biaggi said 250 F and no CO to form 1/3/96

BLOCK VACTRAXI RGIBBS

PARAM TEMP $=550$ PRES $=1<\mathrm{PSI}>$

$\mathrm{PROD} \mathrm{H} 2 \mathrm{O} / \mathrm{H} 2$ / O2 / N2 / S / CL2 / STO2-S S / \& AL2O3-S S / AS / HG / AG / HCL / H2SO4 / CH4 / \& $\mathrm{C}-\mathrm{S}$ S / BA M / CD M/CR M/PBM/SEM/\& FE

PROD-FRAC O2 1

BLOCK VACTRAX2 RGIBBS

PARAM TEMP $=250$ PRES $=1<$ PSI $>$

PROD H2O / H2 / O2 / N2 / S / F2 / SIO2-S S / \&

AL2O3-S S / FE / HG / HCL / C-S S

PROD-FRAC O2 $1 / \mathrm{H} 2 \mathrm{O} 1$

BLOCK VACTRAX3 RGIBBS

PARAM TEMP $=550$ PRES $=1$ <PSI>

PROD H2 / O2 / N2 / CL2 / SIO2-S S / AL2O3-S S / \&

$\mathrm{HCL} / \mathrm{CH} 4 / \mathrm{C}-\mathrm{S} \mathrm{S} / \mathrm{FE} / \mathrm{H} 2 \mathrm{O}$

PROD-FRAC O2 1

DESIGN-SPEC ACID-IN

DEFINE MACD STREAM-VAR STREAM=ACID-IN SUBSTREAM=MIXED \& VARIABLE=MASS-FLOW

DEFINE MASOTM STREAM-VAR STREAM $=$ CWO-OUT SUBSTREAM $=$ MIXED \& VARIABLE=MASS-FLOW

DEFINE MASOTS STREAM-VAR STREAM=CWO-OUT SUBSTREAM=CISOLID \& VARIABLE=MASS-FLOW

DEFINE MASO2 STREAM-VAR STREAM=OXYGEN1 SUBSTREAM=MIXED \& VARIABLE $=$ MASS-FLOW

SPEC "MACID*200 " TO "(MASOTM+MASOTS-MASO2)*7"

TOL-SPEC "0.01"

VARY STREAM-VAR STREAM $=$ ACD-IN SUBSTREAM $=$ MIXED \&

VARIABLE $=$ MASS-FLOW

LIMITS "0.01" "20"

$; 10 \%$ excess $\mathrm{O} 3$

DESIGN-SPEC AIR1

DEFINE OHROUT MOLE-FLOW STREAM=PRODUCTS SUBSTREAM=MIXED \& COMPONENT $=$ OH-RAD

DEFINE OHRIN MOLE-FLOW STREAM=OH-RAD SUBSTREAM=MIXED \& COMPONENT $=O H-R A D$

SPEC "OHROUT* $(1+100 / 10)$ " TO "OHRIN "

TOL-SPEC " 0.05 "

VARY STREAM-VAR STREAM=AIR1 SUBSTREAM $=$ MIXED \& 
VARLABLE=MASS-FLOW

LIMTS "2" "4"

DESIGN-SPEC AIR4

DEFINE OXOUT MASS-FLOW STREAM=GPCR-OUT SUBSTREAM=MIXED \&

COMPONENT $=02$

DEFINE OX1 MASS-FLOW STREAM=AIR4 SUBSTREAM=MIXED \&

COMPONENT $=\mathrm{O} 2$

DEFINE OX2 MASS-FLOW STREAM=FILTERED SUBSTREAM=MIXED \& COMPONENT $=\mathrm{O} 2$

SPEC "OX1+OX2" TO "OXOUT* $(1+100 / 100)$ "

TOL-SPEC " 0.1 "

VARY STREAM-VAR STREAM=AIR4 SUBSTREAM=MIXED \& VARIABLE=MASS-FLOW

LDMTS "0.01" "50"

;Perry's Handbook p. 20-33 has moisture contents about $0.5 \%$ from dryers

;review meeting of $2 / 8 / 96$ : moisture content to $5 \%$

DESIGN-SPEC DRYER

DEFINE H2OMAS MASS-FLOW STREAM=PLYSTBIN SUBSTREAM=MIXED \& COMPONENT $=\mathrm{H} 2 \mathrm{O}$

DEFINE MIXMAS STREAM-VAR STREAM=PLYSTBIN SUBSTREAM=MIXED \& VARIABLE=MASS-FLOW

DEFINE SOLMAS STREAM-VAR STREAM=PLYSTBIN SUBSTREAM=CISOLID \& VARIABLE=MASS-FLOW

SPEC "H2OMAS" TO "(MIXMAS+SOLMAS)*0.05"

TOL-SPEC "0.001"

VARY BLOCK-VAR BLOCK=WATERSEP SENTENCE=FRAC VARIABLE=FRACS \& $\mathrm{ID} 1=\mathrm{MIXED}$ I2 $=$ PLYSTBIN ELEMENT $=10$

LIMITS "0" "1"

;power input is calculated to be $56.49 \mathrm{~W} / \mathrm{SCFM}$ according to information

; supplied by Ben Tehranian, MK Environmental Services

;lbmol/hr converted to SCFM by multiplying by $359 / 60$

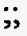

$; 1 \mathrm{~W}=3.413 \mathrm{Btu} / \mathrm{hr}$

DESIGN-SPEC GPCRPOWR

DEFINE MOLOUT STREAM-VAR STREAM=GPCR-OUT SUBSTREAM=MIXED \& VARIABLE=MOLE-FLOW

DEFINE POWER BLOCK-VAR BLOCK=GPCR VARIABLE=DUTY \& SENTENCE $=$ PARAM

SPEC "POWER" TO "MOLOUT*359/60*56.49*3.413"

TOL-SPEC "0.01"

VARY BLOCK-VAR BLOCK=GPCR VARIABLE=DUTY SENTENCE=PARAM

LIMTTS "O" " 1000000 "

; conversation with Daryoush Bahar 3/6/96 - dry basis used for grout calculation ;because with System 3, too much water exists, which results in too much grout ;being calculated 


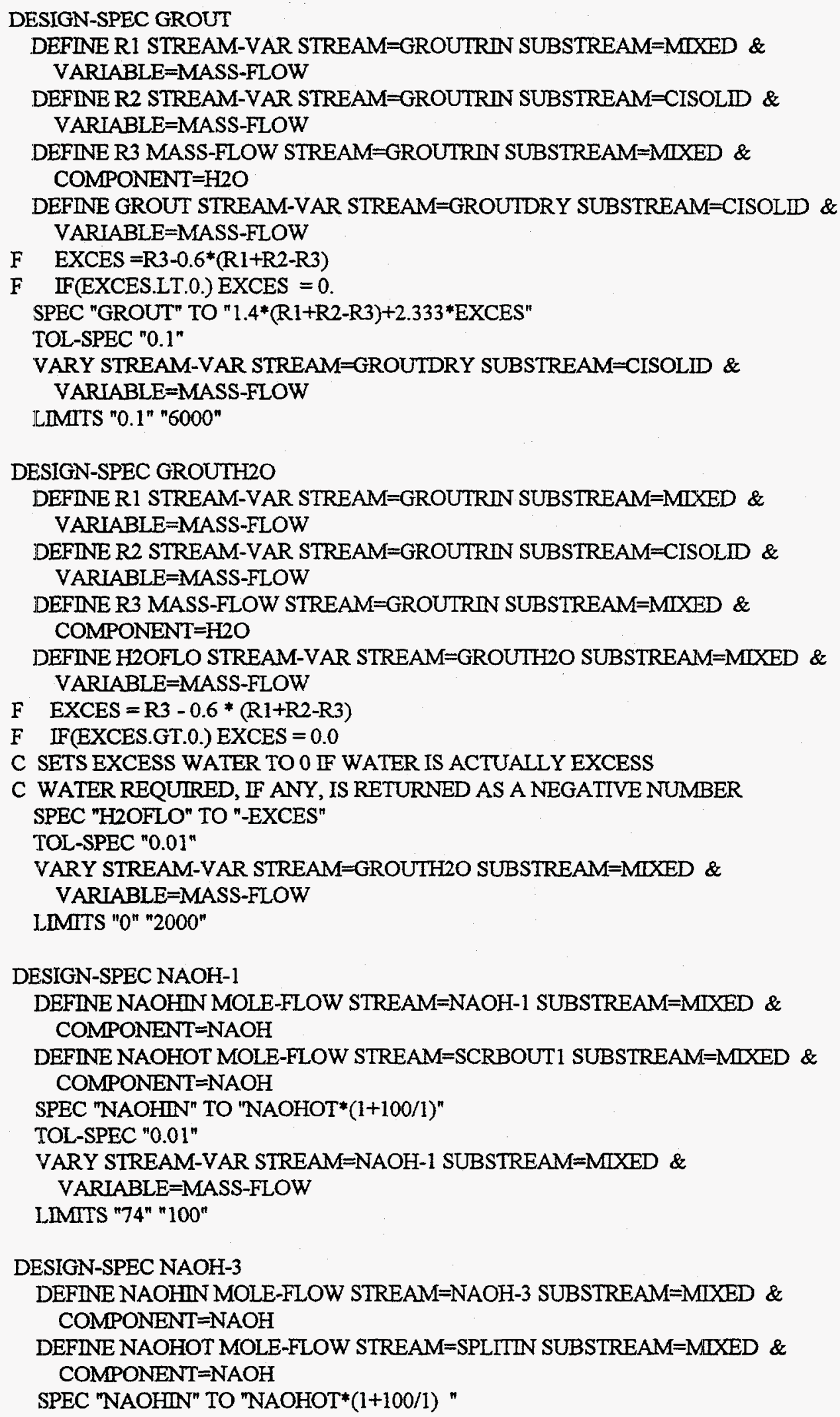




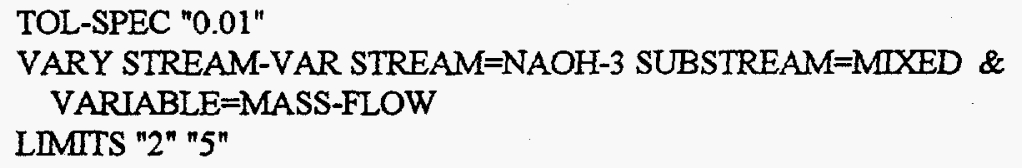




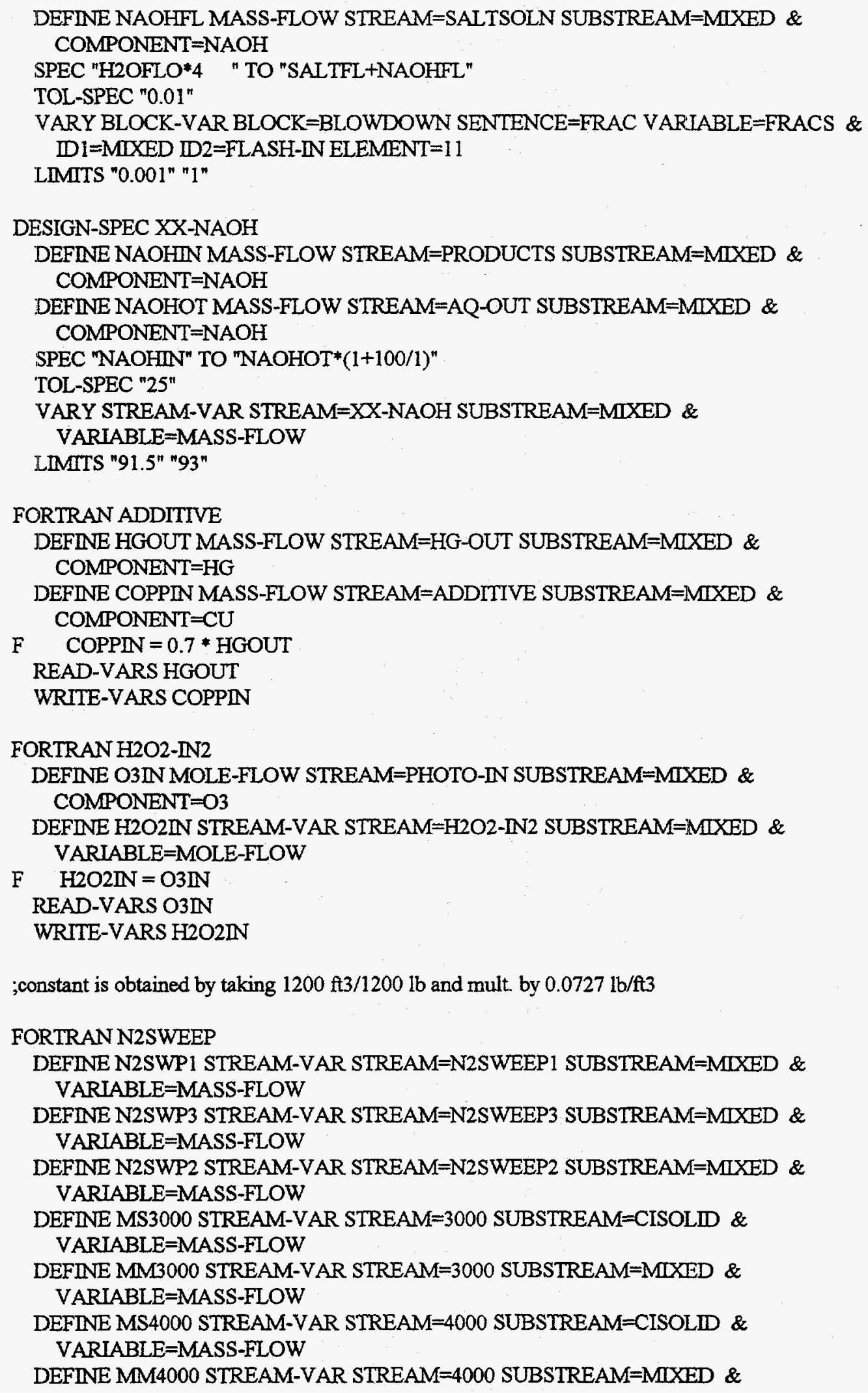


VARIABLE=MASS-FLOW

DEFINE MS5000 STREAM-VAR STREAM $=5000$ SUBSTREAM $=$ CISOLD \& VARIABLE=MASS-FLOW

DEFINE MM5000 STREAM-VAR STREAM=5000 SUBSTREAM=MIXED \&

VARLABLE=MASS-FLOW

F $\quad \mathrm{N} 2 \mathrm{SWP1}=0.0793 *(\mathrm{MS} 3000+\mathrm{MM} 3000)$

F N2SWP2 $=0.0793 *(\mathrm{MS} 5000+\mathrm{MM} 5000)$

F N2SWP3 $=0.0793 *($ MS4000+MM4000)

READ-VARS MS3000 MM3000 MS4000 MM4000 MS5000 MM5000

WRITE-VARS N2SWP1 N2SWP2 N2SWP3

CONV-OPTIONS

PARAM TEAR-METHOD $=$ WEGSTEIN TOL $=0.0021$

STREAM-REPOR MOLEFLOW MASSFLOW

; 


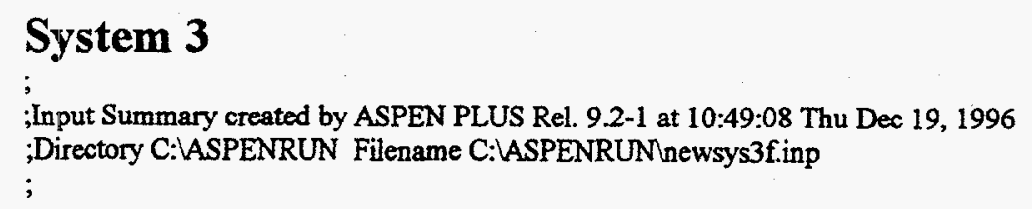


CEMENT CAAL2SIO6 CEMENT /

CASO4 CASO4 CASO4/

AGNO3 AGNO3 AGNO3 /

HNO3 HNO3 HNO3/

HNO2 $\mathrm{HNO} 2 \mathrm{HNO} 2$ /

NO3-NO3-NO3- 1

$\mathrm{H}+\mathrm{H}+\mathrm{H}+1$

E- E-E- 1

$A G+A G+A G+1$

$\mathrm{AG}++\mathrm{AG}+2 \mathrm{AG}++$

$\mathrm{H} 2 \mathrm{O} 2 \mathrm{H}_{2} \mathrm{O} 2 \mathrm{H} 2 \mathrm{O} 2 /$

AGCL AGCL AGCL/

CL- CL- CL- I

NANO3 NANO3 NANO3 /

NH3 H3N NH3/

CU CU CU/

OH-RAD OH OH-RAD

FLOWSHEET APC-SYS

BLOCK GPCR IN=AIR4 FILTERED OUT=GPCR-OUT

BLOCK NEUTRAL 3 IN=NAOH-3 GPCR-OUT OUT=SPLITIN

BLOCK BLOWDOWN IN=SPLITIN OUT=FLASH-IN SALTSOLN

BLOCK FLASH $\mathbb{N}=$ FLASH-DN OUT=HEATERIN MOISTURE

BLOCK FILTER $\mathbb{N}=A Q-O F G A S$ NOX $A G-G A S$ OUT=FLLTERED METALS

BLOCK HETRHEPA $N=$ =HEATERIN OUT=OFFGAS

FLOWSHEET AQUEOUS

BLOCK AQ-SPLIT IN=AQ-OUT OUT=AQ-SLUDG AQ-OFGAS AQ-RECYC

BLOCK OZ-GEN IN=AIRI OUT $=O Z O N E$

BLOCK CONCENT8 IN=SALTSOLN OUT=AQ-RECY2 CONCENT8

BLOCK PHOTOMIX IN=METLRECY 1000 H2ORECYC OZONE MEO-LIQ \& AG-SALTS AQWASTE MOISTURE H2O2-IN2 EXCESH2O ACIDSLUD \& $X X-N A O H$ AQ-RECY2 OUT $=X-P H O T I N$

BLOCK OH-GEN NN=PHOTO-IN OUT $=\mathrm{OH}-\mathrm{RAD}$

BLOCK PHOTO-UV IN=OH-RAD OUT=PRODUCTS

BLOCK OZ-DECOM $\mathbb{N}=$ =PRODUCTS OUT $=A Q-O U T$

BLOCK OILSEP $\mathbb{N}=\mathrm{X}$-PHOTIN OUT=PHOTO-IN X-ORGS

FLOWSHEET BULKSOIL

BLOCK AQ-WASH1 IN=4000 H2OWASH1 OUT=IMM-ORG1 TRETSOL1

BLOCK SOLDRYER IN=TRETSOL1 OUT=WARMSOL1

BLOCK H2OSEP IN=WARMSOL1 OUT=EXCESH2O DRYSOL1

FLOWSHEET GROUT

BLOCK GROUTMIX IN=PBGLOVES WASHSLUD DRYSOLI OUT=GROUTRNN

BLOCK GROUTER IN=GROUTDRY GROUTRIN GROUTH2O OUT=GROUTOUT

FLOWSHEET HG-AMAL

BLOCK HG-AMALG IN=ADDITIVE 7000-HG OUT=HG-OUT

FLOWSHEET METLDCON

BLOCK MET-DCON NN=5000METL METLWASH OUT=METLRECY METALOUT

FLOWSHEET ORGDEST

BLOCK MEO-SPLT IN=SHIFTOUT OUT=MEO-LIQ HNO2 NITRIC-2 \& ACID-OUT H2O-N

BLOCK AGRECOVR IN=NAOH-1 H2O2-IN HNO3-IN1 NITRIC-2 OUT $=$ \& AGRECOVR

BLOCK AG-SPLT $\mathbb{N}=$ =AGRECOVR OUT=AGRECYC AG-SALTS AG-GAS

BLOCK NOX-SPLT IN=HNO2-NO3 OUT=NITRIC-1 X-NOX

BLOCK MEO-MIXR IN=AGNO3-IN 20003000 H2O-IN HNO3-IN2 \&

AGRECYC NITRIC-1 IMM-ORG IMM-ORGI X-ORGS OUT=MEO-IN

BLOCK HNO2RECV IN=HNO2 OXYGEN1 OUT=HNO2-NO3 
BLOCK NEUTRAL IN=ACID-OUT NAOH-4 OUT=ACIDSLUD

BLOCK AG-PPT NN=MEO-OUT HCL-NN OUT=PPT-OUT

BLOCK MEO-3 IN=PPT-OUT OUT=SHIFTOUT

BLOCK MEO-CATH IN=MEO1-OUT OUT=MEO-OUT

BLOCK MEO-ANOD IN=MEO-IN OUT $=$ MEO1-OUT

BLOCK NOX-CONV $\mathrm{N}=\mathrm{X}-\mathrm{NH} 3 \mathrm{X}-\mathrm{NOX}$ OUT $=$ NOX

FLOWSHEET PB-RECVR

BLOCK PB-DECON IN=PBBRICKS PB-WATER OUT=LEAD-OUT PBSLUDGE

BLOCK PB-SPLIT IN=7000-PB OUT=PBBRICKS PBGLOVES

FLOWSHEET POLYMER

BLOCK POLYSTAB IN=PLYSTBIN POLYETHL OUT=POLY-OUT

BLOCK POLY-MIX IN=3000POLY AQ-SLUDG CONCENT8 TRETSOL2 \& METALS OUT=MIXED-IN

BLOCK DRYER IN=MLXED-IN OUT=HOT-MIX

BLOCK WATERSEP NN=HOT-MTX OUT=PLYSTBIN H2ORECYC

FLOWSHEET RES-SLUD

BLOCK AQ-WASH4 IN=H2OWASH4 5000OPEN OUT=IMM-ORG4 TRETSOL4 \& AQWASTEA

BLOCK AQ-WASH2 $\mathrm{N}=\mathrm{H} 2$ OWASH2 3000 PBSLUDGE OUT=IMM-ORG2 \& TRETSOL2 AQWASTE2

BLOCK ORG-MIXR IN=IMM-ORG4 IMM-ORG3 IMM-ORG2 IMM-ORG5 OUT= \& IMM-ORG

BLOCK AQU-MLXR IN=AQWASTE3 AQWASTE4 AQWASTE2 AQWASTE5 OU $\Gamma=\&$ AQWASTE

BLOCK SOL-MIXR IN=TRETSOL3 TRETSOLA TRETSOL5 OUT=WASHSLUD

BLOCK AQ-WASH5 IN=H2OWASH5 5000COMP OUT=IMM-ORG5 TRETSOLS \& AQWASTES

BLOCK AQ-WASH3 $\mathrm{IN}=$ H2OWASH3 5000SOFT OUT=IMM-ORG3 TRETSOL3 \& AQWASTE3

FLOWSHEET SPECLAL

BLOCK SPESHULL $\mathbb{N}=6000$ OUT $=$ SPEC-OUT

PROPERTIES SOLIDS

PROPERTIES IDEAL

USER-PROPS DRUSR2 123

PROP-DATA U-2

IN-UNITS ENG PRESSURE $=$ ATM

PROP-LIST TC

PVAL HNO2 100

PROP-DATA U-1

IN-UNITS ENG PRESSURE=ATM

PROP-LIST PLXANT

PVAL HNO2 7.511-1406 221

PROP-DATA U-1

IN-UNITS ENG PRESSURE=ATM

PROP-LIST DHVLWT

PVAL HNO2 11

;Tables dated 04/24/96 from Daryoush Bahar, MK, (415) 442-7688

;Glass inerts assumed to be $50 \% \mathrm{SiO} 2$ and $50 \% \mathrm{Al} 2 \mathrm{O} 3$ for all waste streams.

STREAM 1000

SUBSTREAM MIXED TEMP $=68$ PRES $=1$ MASS-FLOW $=60.8$

MASS-FLOW H2 $3613<$ KG/HR $>/ 02172053<$ KG/HR $>$ / N2 \& $50211<\mathrm{KG} / \mathrm{HR}>/ \mathrm{H} 202456410<\mathrm{KG} / \mathrm{HR}>/ \mathrm{AS} 5647<\mathrm{KG} / \mathrm{HR}>\&$ 
/ BA $2823<\mathrm{KG} / \mathrm{HR}>$ / CD $42352<\mathrm{KG} / \mathrm{HR}>$ / CR \& $56469<\mathrm{KG} / \mathrm{HR}>/ \mathrm{PB} 14117<\mathrm{KG} / \mathrm{HR}>/ \mathrm{HG} 14117<\mathrm{KG} / \mathrm{HR}>/$ \& SE $2823<\mathrm{KG} / \mathrm{HR}>/$ AG $2823<\mathrm{KG} / \mathrm{HR}>$

;Tables dated 04/24/96 from MK

STREAM 3000 SUBSTREAM MIXED TEMP $=68$ PRES $=1$ MASS-FLOW=352.12 MASS-FLOW H2 $268596<\mathrm{KG} / \mathrm{HR}>/ \mathrm{O} 210180<\mathrm{KG} / \mathrm{HR}>/ \mathrm{N} 2 \&$ $122<\mathrm{KG} / \mathrm{HR}>$ / S $6233<\mathrm{KG} / \mathrm{HR}>$ / CL2 $383185<\mathrm{KG} / \mathrm{HR}>$ / \& H2O $18588527<\mathrm{KG} / \mathrm{HR}>$ / FE $107084<\mathrm{KG} / \mathrm{HR}>$ / AS \& $3383<$ KG/HR> / BA $12700<\mathrm{KG} / \mathrm{HR}>/ \mathrm{CD} 158485<\mathrm{KG} / \mathrm{HR}>$ / \& CR $299848<\mathrm{KG} / \mathrm{HR}>/ \mathrm{PB} 9640<\mathrm{KG} / \mathrm{HR}>/ \mathrm{HG} 8445<\mathrm{KG} / \mathrm{HR}>$ \& / SE $1689<\mathrm{KG} / \mathrm{HR}>$ / AG $1693<\mathrm{KG} / \mathrm{HR}>$ SUBSTREAM CISOLID TEMP=68 PRES=1 MASS-FLOW $=655.08$ MASS-FLOW C-S $1658863<\mathrm{KG} / \mathrm{HR}>$ / SIO2-S $17644467<\mathrm{KG} / \mathrm{HR}>$ / \& Al2O3-S $17644467<$ KG/HR>

;tables dated 04/24/96 from MK

STREAM 3000POLY SUBSTREAM MIXED TEMP=68 PRES=1 MASS-FLOW $=3.28$ MASS-FLOW CL2 $98888<\mathrm{KG} / \mathrm{HR}>$ / NO $37279<\mathrm{KG} / \mathrm{HR}>/ \mathrm{SO} 2$ \& $47075<\mathrm{KG} / \mathrm{HR}>$ SUBSTREAM CISOLID TEMP $=68$ PRES $=1$ MASS-FLOW $=3.32$ MASS-FLOW SIO2-S 92796 / AL2O3-S 92796

;tables dated 04/24/96 from MK

STREAM 4000

SUBSTREAM MIXED TEMP $=68$ PRES $=1$ MASS-FLOW $=40.74$

MASS-FLOW H2 $21653<\mathrm{KG} / \mathrm{HR}>/ \mathrm{CL} 2761580<\mathrm{KG} / \mathrm{HR}>/ \mathrm{H} 2 \mathrm{O}$ \& $1514279<\mathrm{KG} / \mathrm{HR}>$ SUBSTREAM CISOLID TEMP $=68$ PRES $=1$ MASS-FLOW $=287.76$ MASS-FLOW C-S $175090<\mathrm{KG} / \mathrm{HR}>/$ SIO2-S $8027287.5<\mathrm{KG} / \mathrm{HR}>/$ \& AL2O3-S 8027287.5 <KG/HR>

;Data table dated 04/24/96. Supplied by Daryoush Bahar, MK (415) 442-7688

STREAM 5000COMP SUBSTREAM MIXED TEMP $=68$ PRES $=1$ MASS-FLOW H2 3.026 / H2O 0.197 / FE $148.890 / \mathrm{HG} 1.3$ SUBSTREAM CISOLD TEMP $=68$ PRES $=1$ MASS-FLOW C-S 17.992 / SIO2-S 3.003 / AL2O3-S 3.003

;tables dated 04/24/96 from MK

STREAM 5000METL SUBSTREAM MIXED TEMP=68 PRES=1 MASS-FLOW=434.82 MASS-FLOW H2 141789 / FE 13383118 SUBSTREAM CISOLID TEMP $=68$ PRES $=1$ MASS-FLOW $=27.29$ MASS-FLOW C-S 844686 / SIO2-S 1981 / AL2O3-S 1981

;tables dated 04/24/96 from MK

STREAM S000OPEN

SUBSTREAM MIXED TEMP $=68$ PRES $=1$ MASS-FLOW $=0.40$ MASS-FLOW H2 $4587<\mathrm{KG} / \mathrm{HR}>/ \mathrm{O} 218201<\mathrm{KG} / \mathrm{HR}>$ SUBSTREAM CISOLID TEMP=68 PRES $=1$ MASS-FLOW=80 MASS-FLOW C-S $22543<\mathrm{KG} / \mathrm{HR}>$ / SIO2-S $2243854<\mathrm{KG} / \mathrm{HR}>$ / \& AL2O3-S $2243854<\mathrm{KG} / \mathrm{HR}>$

;tables dated 04/30/96, used mass flow $\mathrm{lb} / \mathrm{hr}$ rather than $\mathrm{kg}$ in waste inventory 


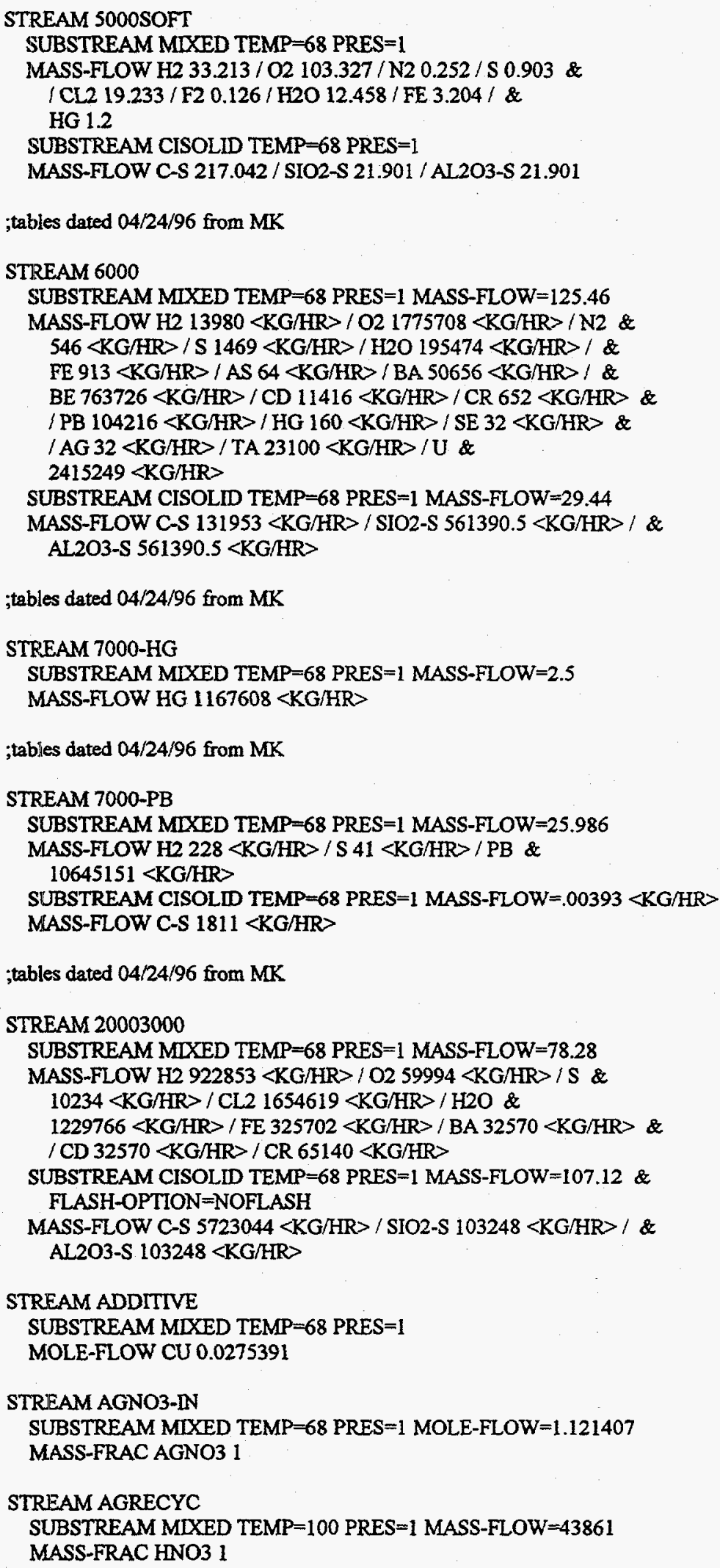


STREAM AIR1

SUBSTREAM MDXED TEMP=68 PRES=1 MASS-FLOW=5200

MOLE-FRAC O2 $0.21 / \mathrm{N} 20.79$

STREAM AIR4

SUBSTREAM MIXED TEMP=68 PRES=1 MASS-FLOW=0.001

MOLE-FRAC O2 0.21/N2 0.79

STREAM GROUTDRY

SUBSTREAM MIXED TEMP $=68$ PRES $=1$

SUBSTREAM CISOLID TEMP $=68$ PRES $=1$ MASS-FLOW $=4127.367$

MASS-FRAC CEMENT 1

STREAM GROUTH2O

SUBSTREAM MIXED TEMP $=68$ PRES $=1$ MASS-FLOW $=0.000001$

MASS-FRAC H2O 1

STREAM H2O-IN

SUBSTREAM MLXED TEMP $=68$ PRES $=1$ MASS-FLOW $=0.000001$

MASS-FRAC $\mathrm{H} 2 \mathrm{O} 1$

STREAM H2O2-DN

SUBSTREAM MIXED TEMP=68 PRES=1 MASS-FLOW=1

MASS-FRAC $\mathrm{H} 2 \mathrm{O} 21$

STREAM H2O2-IN2

SUBSTREAM MIXED TEMP $=68$ PRES $=1$ MOLE-FLOW $=1$ MASS-FRAC $\mathrm{H} 2 \mathrm{O} 21$

STREAM H2OWASHI

SUBSTREAM MIXED TEMP=68 PRES=1 MASS-FLOW=328.5

MASS-FRAC H2O 1

;water flow rate reduced to $15 \%$ of waste input

STREAM H2OWASH2

SUBSTREAM MIXED TEMP=68 PRES=1 MASS-FLOW=151.05

MASS-FRAC H2O

;water flow rate reduced to $15 \%$ of waste input, $10 \%$ to recycle, $5 \%$ with waste

STREAM H2OWASH3

SUBSTREAM MTXED TEMP=68 PRES=1 MASS-FLOW=65.25

MASS-FRAC H2O

;amount of water set to be $15 \%$ of waste input, $10 \%$ to recycle, $5 \%$ with waste

STREAM H2OWASH4

SUBSTREAM MIXED TEMP=68 PRES=1 MASS-FLOW=12

MASS-FRAC $\mathrm{H} 2 \mathrm{O} 1$

;water flow rate reduced to $15 \%$ of waste input, $10 \%$ to recycle, $5 \%$ with waste

STREAM H2OWASHS

SUBSTREAM MIXED TEMP=68 PRES=1 MASS-FLOW=26.55

MASS-FRAC $\mathrm{H} 2 \mathrm{O}_{1}$

STREAM HCL-IN

SUBSTREAM MIXED TEMP=68 PRES=1 MASS-FLOW=1

MASS-FRAC HCL 1

STREAM HG-OUT

SUBSTREAM MIXED TEMP $=68$ PRES $=1$ 
MOLE-FLOW HG 0.0124632, CU 0.0275391

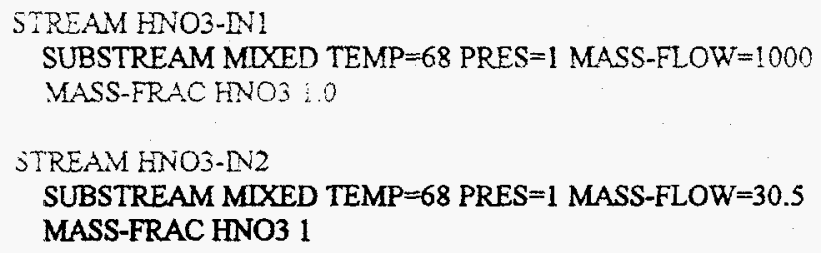

STREAM NITRIC-2 SUBSTREAM MIXED TEMP $=68$ PRES $=1$ MASS-FLOW $=100$ MASS-FRAC HNO3 1

STREAM OXYGEN1 SUBSTREAM MIXED TEMP $=68$ PRES $=1$ MOLE-FLOW=435 MOLE-FLOW O2 1

STREAM PB-WATER SUBSTREAM MIXED TEMP=68 PRES=1 MASS-FLOW=13 MASS-FRAC $\mathrm{H} 2 \mathrm{O} 1$

;Polymer assumed to be polyethylene; $\mathrm{C} 2 \mathrm{H} 4$ used as the monomer.

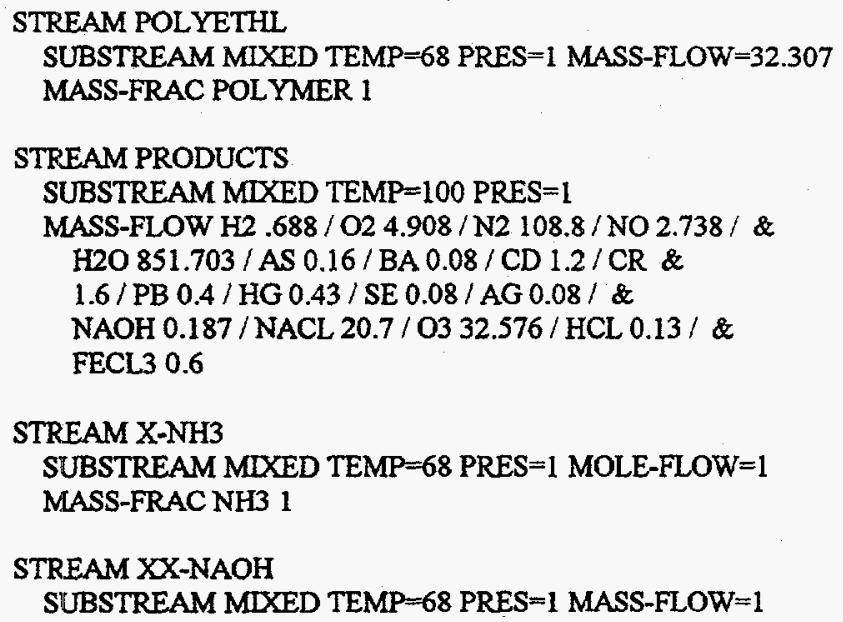


MASS-FRAC H2O 0.5 / NAOH 0.5

BLOCK AQU-MIXR MLXER

PARAM PRES $=1$

BLOCK GROUTER MIXER

PARAM PRES $=1$ NPHASE $=2 \mathrm{MAXIT}=500 \mathrm{TOL}=0.01$

BLOCK GROUTMIX MDXER

PARAM PRES $=1$

BLOCK HG-AMALG MIXER

BLOCK MEO-MIXR MIXER

PARAM PRES $=1$ MAXIT $=99$

BLOCK ORG-MIXR MIXER

PARAM PRES $=1$

BLOCK PHOTOMIX MIXER

BLOCK POLY-MIX MIXER

PARAM MAXIT $=100$

BLOCK POLYSTAB MIXER

BLOCK-OPTION ENERGY-BAL =YES

BLOCK SOL-MIXR MLXER

PARAM PRES $=1$ MAXIT $=500$

BLOCK AG-SPLT SEP

PARAM PRES $=1$

FRAC STREAM=AGRECYC SUBSTREAM=MIXED COMPS=C C-S H2 O2 \& N2 S CL2 F2 NO NO2 H2O SIO2 SIO2-S AL2O3 AL2O3-S \& FE AS BA CD CR PB HG SE AG NAOH NACL CO2 $\mathrm{CO} O 3$ \&

HCL FECL3 H2SO4 NA2SO4 CH4 POLYMER CEMENT CASO4 AGNO3 \& HNO3 $\mathrm{HNO} 2 \mathrm{NO} 3-\mathrm{H}+\mathrm{E}-\mathrm{AG}+\mathrm{AG}++\mathrm{H} 2 \mathrm{O} 2 \mathrm{AGCL} \mathrm{CL}-\mathrm{NANO} 3$ \&

NH3 CU FRACS $=000000000000000$ \&

$000000000000000000000 \&$

011000000000000

FRAC STREAM =AGRECYC SUBSTREAM=CISOLID COMPS=C-S SIO2-S \& AL2O3-S CEMENT FRACS $=0000$

FRAC STREAM $=A G-G A S$ SUBSTREAM=MLXED COMPS $=\mathrm{C} C-\mathrm{S} \mathrm{H} 2 \mathrm{O} 2 \mathrm{~N} 2$ \& S CL2 F2 NO H2O SIO2 SIO2-S AL2O3 AL2O3-S FE AS BA \& CD CR PB HG SE AG NAOH NACL $\mathrm{CO} 2 \mathrm{CO} O 3 \mathrm{HCL}$ FECL3 \& $\mathrm{H} 2 \mathrm{SO} 4 \mathrm{NA} 2 \mathrm{SO} 4 \mathrm{CH} 4 \mathrm{POLYMER}$ CEMENT CASO4 AGNO3 HNO3 $\mathrm{HNO} 2$ \& NO3-H+E- AG+ AG++ H2O2 AGCL FRACS $=001110$ \&

110000000000000000011 \&

1000010000000000000

FRAC STREAM=AG-GAS SUBSTREAM=CISOLID COMPS=C-S SIO2-S \& AL2O3-S CEMENT FRACS $=0000$

FLASH-SPECS AGRECYC TEMP $=100$ PRES $=1$

FLASH-SPECS AG-SALTS TEMP $=100$ PRES $=1$ NPHASE $=1$

FLASH-SPECS AG-GAS TEMP $=100$ PRES $=1$

BLOCK AQ-SPLIT SEP

DESCRIPTION "this separates water, gases, and solids"

FRAC STREAM=AQ-SLUDG SUBSTREAM=MIXED COMPS $=\mathrm{C} C-\mathrm{S}$ H2 $\mathrm{O} 2$ \& N2 S CL2 F2 NO SO2 H2O SIO2 SIO2-S AL2O3 AL2O3-S \& FE AS BA CD CR PB HG SE AG NAOH NACL CO2 $\mathrm{CO} O 3$ \& HCL FECL3 H2SO4 NA2SO4 CH4 POLYMER CEMENT CASO4 AGNO3 \& HNO3 HNO2 NO3- $\mathrm{H}+\mathrm{E}-\mathrm{AG}+\mathrm{AG}++\mathrm{H} 2 \mathrm{O} 2 \mathrm{AGCL} \mathrm{CL}-\mathrm{NANO} 3$ \& FRACS $=110001000000.990 .990 .99 \&$ 
$0.991111111111100000010 \&$

111100000000101

FRAC STREAM=AQ-SLUDG SUBSTREAM=CISOLID COMPS=C-S SIO2-S \& AL2O3-S CEMENT FRACS $=0.990 .990 .990 .99$

FRAC STREAM=AQ-OFGAS SUBSTREAM=MIXED COMPS=C C-S H2 O2 \& N2 S CL2 $\mathrm{F} 2$ NO NO2 SO2 H2O SIO2 SIO2-S AL2O3 \&

AL2O3-S FE AS BA CD CR PB HG SE AG NAOH NACL CO2 \& $\mathrm{CO} \mathrm{O} 3 \mathrm{HCL}$ FECL $3 \mathrm{H} 2 \mathrm{SO} 4 \mathrm{NA} 2 \mathrm{SO} 4 \mathrm{CH} 4$ POLYMER CEMENT \& CASO4 AGNO3 HNO3 HNO2 NO3- $\mathrm{H}+\mathrm{E}-\mathrm{AG}+\mathrm{AG}++\mathrm{H} 2 \mathrm{O} 2 \mathrm{AGCL} \&$ CL-NANO3 NH3 FRACS $=0011101110100$ \&

000000000000001110000 \&

10000000000000000

FRAC STREAM=AQ-OFGAS SUBSTREAM=CISOLID COMPS=C-S SIO2-S \& AL2O3-S CEMENT FRACS $=0000$

\section{BLOCK AQ-WASH1 SEP \\ FRAC STREAM=IMM-ORG1 SUBSTREAM=MIXED COMPS=H2 CL2 $\mathrm{H} 2 \mathrm{O}$ \& FRACS $=0.950 .950$ \\ FRAC STREAM $=$ IMM-ORG1 SUBSTREAM $=$ CISOLID COMPS=C-S SIO2-S \& AL2O3-S CEMENT FRACS $=0.95000$}

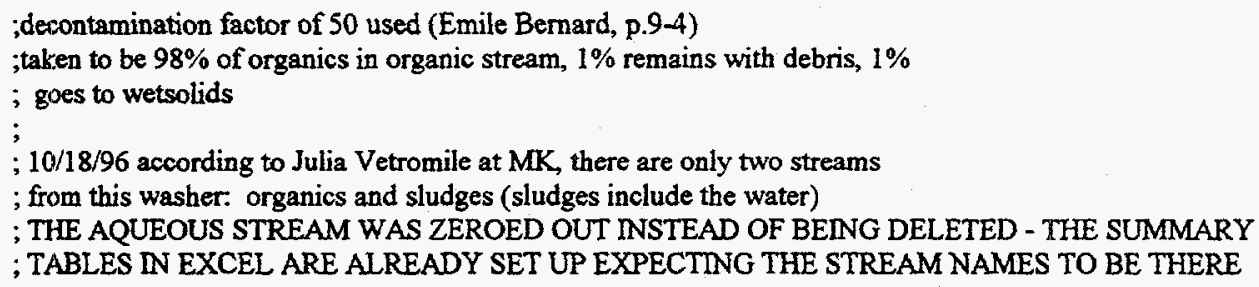

FRAC STREAM=AQWASTE2 SUBSTREAM=CISOLID COMPS=C-S SIO2-S \& AL203-S CEMENT FRACS $=0000$

;decontamination factor of 50 used (Emile Bernard, p.9-4)

;taken to be $98 \%$ of organics in organic stream, $1 \%$ remains with debris, $1 \%$

; goes to wetsolids

; conversation with Bill Schwinkendorf 12/20/95 reveals that for SOFT debris ; (paper, cardboard, rags, etc.) the amount of hydrocarbons removed from the ; debris will be minimal. $10 \%$ assumed.

;

;6/24/96 according to Figure B3-7 from $\mathrm{MK}$, there are only two streams:

; wesshed debris and aqueous organics.

;

;10/18 all organics put in with aqueous stream. ORGANICS STREAM ZEROED OUT ;INSTEAD OF BEING DELETED; POST-PROCESSING BY EXCEL EXPECTS THE STREAM NAME

;wash water routed to aqueous waste $(2 / 3)$ and to solids (1/3) 


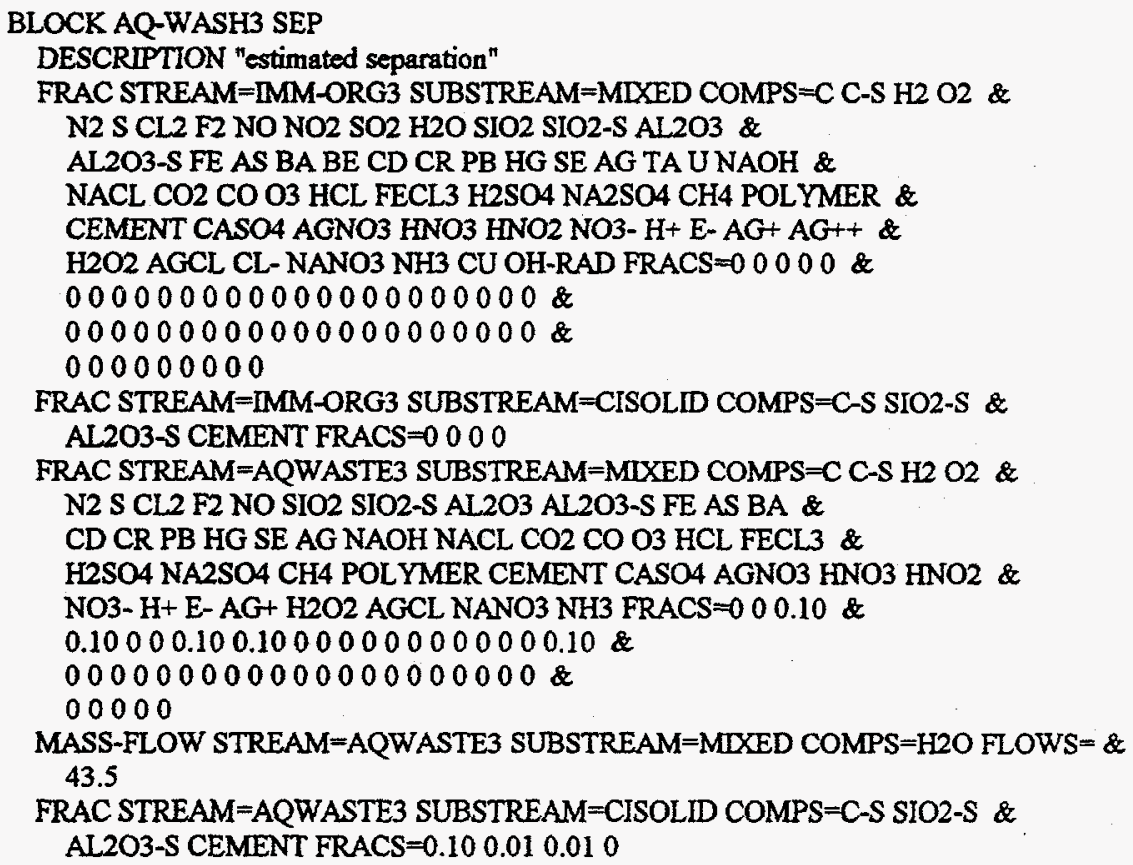

FRAC STREAM=IMM-ORG3 SUBSTREAM=CISOLID COMPS=C-S SIO2-S \& AL2O3-S CEMENT FRACS $=0000$

FRAC STREAM=AQWASTE3 SUBSTREAM=MIXED COMPS $=\mathrm{C}$ C-S $\mathrm{H} 2 \mathrm{O} 2$ \& N2 S CL2 F2 NO SIO2 SIO2-S AL2O3 AL2O3-S FE AS BA \& CD CR PB HG SE AG NAOH NACL CO2 CO O3 HCL FECL3 \& H2SO4 NA2SO4 CH4 POLYMER CEMENT CASO4 AGNO3 HNO3 HNO2 \& NO3- H+E- AG+ H2O2 AGCL NANO3 NH3 FRACS $=000.10$ \& $0.10000 .100 .10000000000000 .10 \&$ $000000000000000000000 \&$ 00000

MASS-FLOW STREAM=AQWASTE3 SUBSTREAM=MIXED COMPS=H2O FLOWS $=$ \& 43.5

FRAC STREAM=AQWASTE3 SUBSTREAM=CISOLID COMPS=C-S SIO2-S \& AL2O3-S CEMENT FRACS $=0.100 .010 .010$

; decontamination factor of 50 used (Emile Bernard, p.9-4)

;taken to be $98 \%$ of organics in organic stream, $1 \%$ remains with debris, $1 \%$

; goes to wetsolids

;according to Julia Vetromile, solids contain 1:1 ratio of water ( $1 \%$ left over)

;3/8/96 Julia said that open and complex debris would have $\sim 5 \%$ water entrain

;3 3/29/96 changed to be the same as HPWash for systems 4 \& 5, immorg stream ; zeroed out

;8/19/96 agreed with Julia to have $15 \%$ of debris mass as make-up water, with

\#2/3 of water going to aqueous waste, $1 / 3$ going with solids

;10/18 all organics put with aqueous stream. ORGANIC STREAM NOT DELETED; ;POST-PROCESSING BY EXCEL EXPECTS STREAM NAME IN EXPORTED FILE.

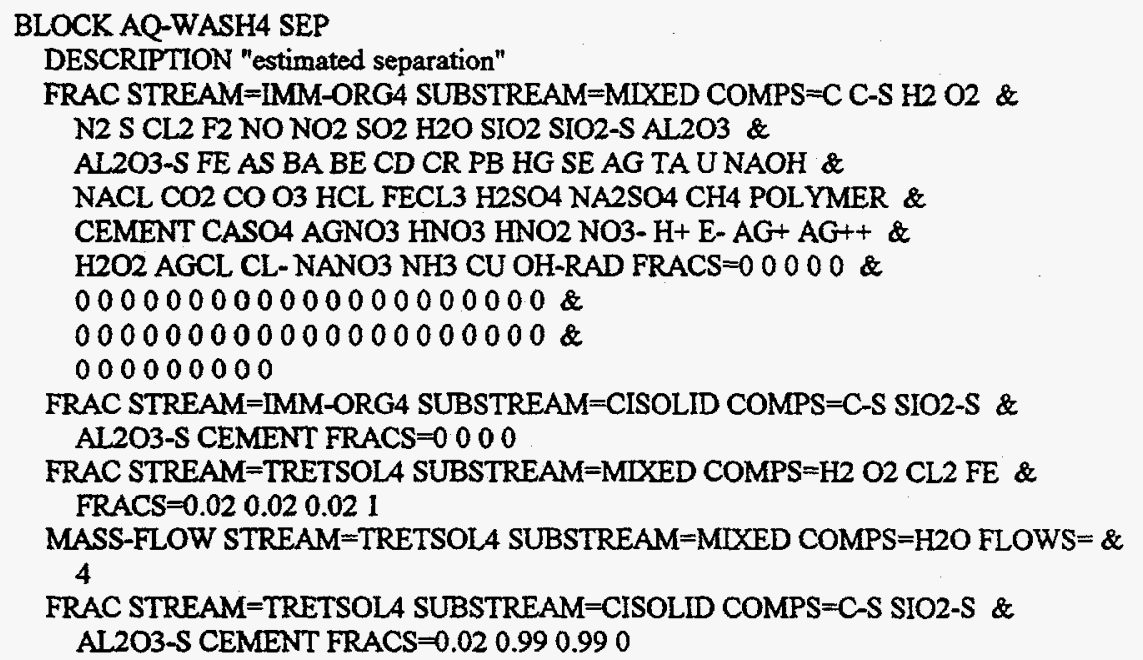

;decontamination factor of 50 used (Emile Bernard, p.9-4)

;taken to be $98 \%$ of organics in organic stream, $1 \%$ remains with debris, $1 \%$

; goes to wetsolids

;3/8/96 Julia Vetromile said that complex, open debris would have $\sim 5 \%$ entrain. 


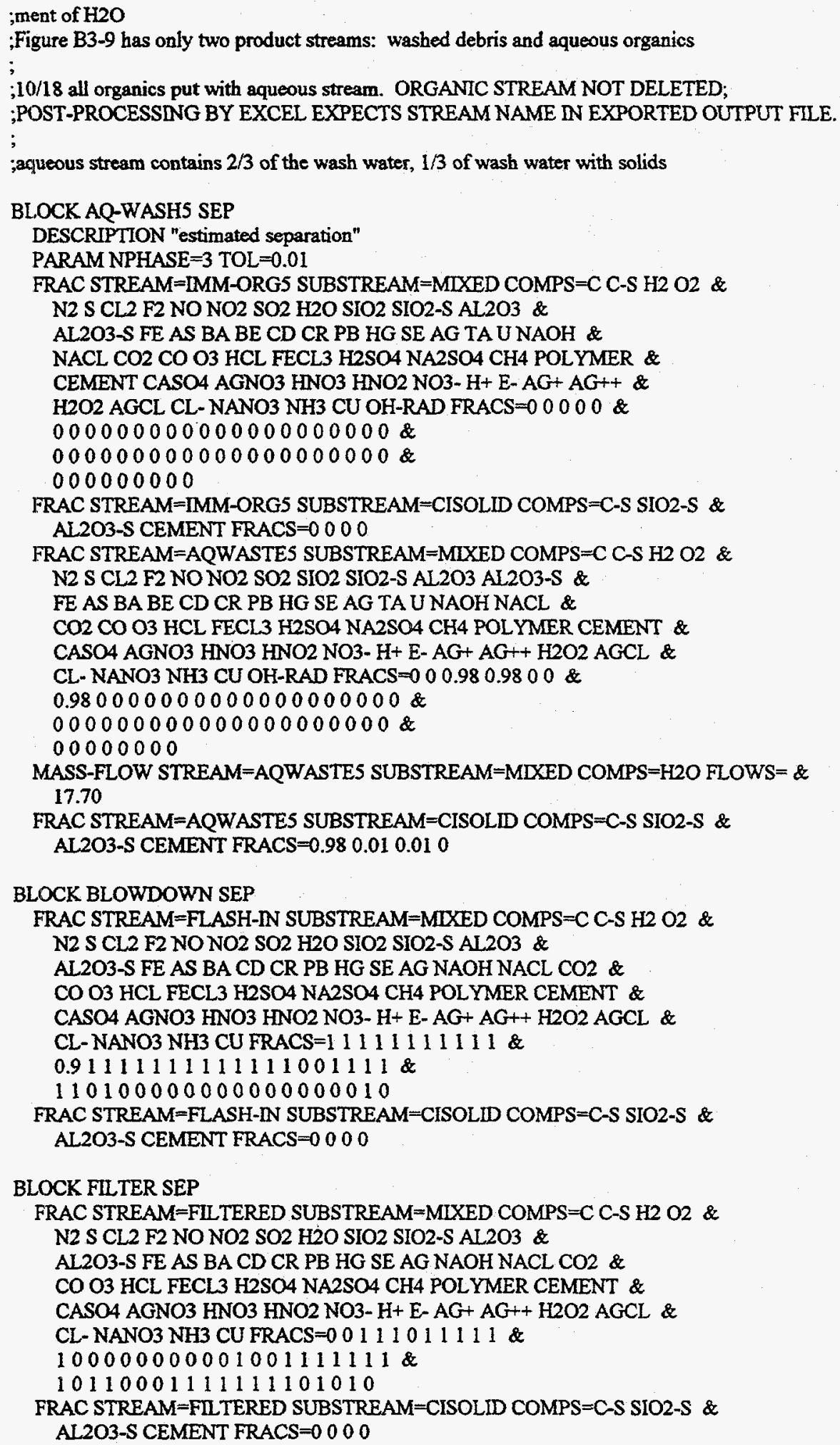

;This is slightly different than the corresponding subsystems in \#4 and \#5, ;but all of the consituents are bound for the same downstream processing. 
;according to note from Julia $6 / 26 / 96$, no aqueous waste stream from soil wash, ; this makes sense with grout especially (why dry soil, only to add water later

BLOCK H2OSEP SEP

DESCRIPTION "separates remaining $\mathrm{H} 2 \mathrm{O}$ from washed soil"

FRAC STREAM=EXCESH2O SUBSTREAM=MIXED COMPS $=\mathrm{C} C \mathrm{C}-\mathrm{S}$ H2 $\mathrm{O}$ \& N2 S CL2 F2 NO NO2 SO2 $\mathrm{H}_{2} \mathrm{O} \mathrm{SIO}_{2} \mathrm{SIO}_{2} \mathrm{~S} \mathrm{AL} 2 \mathrm{O} 3$ \& AL203-S FE AS BA CD CR PB HG SE AG NAOH NACL $\mathrm{CO} 2 \&$ $\mathrm{CO} O 3 \mathrm{HCL}$ FECL 3 H2SO4 NA2SO4 CH4 POLYMER CEMENT \& $\mathrm{CASO} 4 \mathrm{AGNO} 3 \mathrm{HNO} 3 \mathrm{HNO} 2 \mathrm{NO} 3-\mathrm{H}+\mathrm{E}-\mathrm{AG}+\mathrm{AG}++\mathrm{H} 2 \mathrm{O} 2 \mathrm{AGCL} \&$ CL-NANO3 NH3 CU OH-RAD FRACS $=000000000 \&$ $00100000000000.05000000 \&$ $000000000000000000000 \&$ 000

FRAC STREAM=EXCESH2O SUBSTREAM=CISOLID COMPS=C-S SIO2-S \& AL2O3-S CEMENT FRACS $=0000$

;Hg retention in solid set to 0.90 to allow some in gas phase

\section{BLOCK MEO-SPLT SEP}

DESCRIPTION "for the moment, assumes complete separation"

FRAC STREAM=MEO-LIQ SUBSTREAM=MIXED COMPS=C C-S H2 O2 \& N2 S CL2 F2 NO NO2 H2O SIO2 SIO2-S AL2O3 AL2O3-S \& FE AS BA CD CR PB HG SE AG NAOH NACL CO2 $\mathrm{CO} \mathrm{O} 3$ \& $\mathrm{HCL}$ FECL3 H2SO4 NA2SO4 CH4 POLYMER CEMENT CASO4 AGNO3 \& HNO3 HNO2 NO3- $\mathrm{H}+\mathrm{E}-\mathrm{AG}+\mathrm{AG}++\mathrm{H} 2 \mathrm{O} 2$ AGCL FRACS $=00$ \& 100010000.3190000000000 \& $000000001010000000001 \&$ 00000

FRAC STREAM=MEO-LIQ SUBSTREAM=CISOLID COMPS=C-S SIO2-S \& AL2O3-S CEMENT FRACS $=0000$

FRAC STREAM=HNO2 SUBSTREAM=MIXED COMPS=C C-S H2 O2 N2 \& S CL2 F2 NO H2O SIO2 SIO2-S AL2O3 AL2O3-S FE AS BA \& CD CR PB HG SE AG NAOH NACL CO2 $\mathrm{CO} O 3 \mathrm{HCL}$ FECL3 \& H2SO4 NA2SO4 CH4 POLYMER CEMENT CASO4 AGNO3 HNO3 HNO2 \& $\mathrm{NO} 3-\mathrm{H}+\mathrm{E}-\mathrm{AG}+\mathrm{AG}++\mathrm{H} 2 \mathrm{O} 2 \mathrm{AGCL}$ FRACS $=0001100$ \& 000000000000000000010 \& 0000000000010000000

FRAC STREAM $=$ HNO2 SUBSTREAM=CISOLID COMPS=C-S SIO2-S \& AL2O3-S CEMENT FRACS $=0000$

FRAC STREAM $=$ NITRIC-2 SUBSTREAM=MIXED COMPS $=\mathrm{C}$ C-S $\mathrm{H} 2 \mathrm{O} 2 \&$ N2 S CL2 F2 NO H2O SIO2 SIO2-S AL2O3 AL2O3-S FE AS \& BA CD CR PB HG SE AG NAOH NACL CO2 CO O3 HCL \& FECL3 H2SO4 NA2SO4 CH4 POLYMER CEMENT CASO4 AGNO3 \& $\mathrm{HNO} 3 \mathrm{HNO} 2 \mathrm{NO} 3-\mathrm{H}+\mathrm{E}-\mathrm{AG}+\mathrm{AG}++\mathrm{H} 2 \mathrm{O} 2 \mathrm{AGCL} \mathrm{CL}-\mathrm{FRACS}=$ \& 000000000000000000000 \& $00000000000000010.9999900 \&$ 0111010

FRAC STREAM=NITRIC-2 SUBSTREAM=CISOLID COMPS=C-S SIO2-S \& AL2O3-S CEMENT FRACS $=0000$

FRAC STREAM=ACID-OUT SUBSTREAM=MIXED COMPS=F2 FE BA CD \& CR HNO3 FRACS $=111110.00001$

FRAC STREAM=ACID-OUT SUBSTREAM=CISOLID COMPS=C-S SIO2-S \& AL2O3-S CEMENT FRACS=1 111

\section{BLOCK MET-DCON SEP}

DESCRIPTION "Separates water from metal"

FRAC STREAM $=$ METLRECY SUBSTREAM=MIXED COMPS=C C-S H2 O2 \& N2 S CL2 F2 NO H2O SIO2 SIO2-S AL2O3 AL2O3-S FE AS \& BA CD CR PB HG SE AG NAOH NACL CO2 CO HCL FECL3 \& H2SO4 NA2SO4 CH4 POLYMER CEMENT FRACS $=001000$ \& 000100000000000000000 \& 0000000 
FRAC STREAM=METLRECY SUBSTREAM=CISOLID COMPS=C-S SIO2-S \& AL2O3-S CEMENT FRACS $=1110$

BLOCK NOX-SPLT SEP

FRAC STREAM=NITRIC-1 SUBSTREAM=MIXED COMPS=C C-S H2 $\mathrm{O} 2$ \& N2 S CL2 F2 NO NO2 H2O SIO2 SIO2-S AL2O3 AL2O3-S \&

FE AS BA CD CR PB HG SE AG NAOH NACL CO2 $\mathrm{CO} O 3$ \& HCL FECL3 H2SO4 NA2SO4 CH4 POLYMER CEMENT CASO4 AGNO3 \& HNO3 HNO2 NO3- $\mathrm{H}+\mathrm{E}-\mathrm{AG}+\mathrm{AG}+\mathrm{H} 2 \mathrm{O} 2$ AGCL CL-NANO3 \& NH3 CU FRACS $=000000000000000 \&$ 000000000000000000000 \& 001000000000000

FRAC STREAM=NITRIC-1 SUBSTREAM=CISOLID COMPS=C-S SIO2-S \& AL2O3-S CEMENT FRACS $=0 \quad 000$

BLOCK OLSEP SEP

DESCRIPTION "assumes $99 \%$ separation of organics $(\mathrm{C}, \mathrm{H}, \mathrm{CHx})$ "

FRAC STREAM=PHOTO-IN SUBSTREAM=MIXED COMPS $=\mathrm{C} C-\mathrm{S} \mathrm{H} 2 \mathrm{O} 2$ \& N2 S CL2 F2 NO NO2 SO2 $\mathrm{H} 2 \mathrm{O} \mathrm{SIO}_{2} \mathrm{SIO}_{2}-\mathrm{S} \mathrm{AL} 2 \mathrm{O} 3$ \& AL2O3-S FE AS BA BE CD CR PB HG SE AG TA U NAOH \& NACL $\mathrm{CO} 2 \mathrm{CO} O 3 \mathrm{HCL}$ FECL $3 \mathrm{H} 2 \mathrm{SO} 4 \mathrm{NA} 2 \mathrm{SO} 4 \mathrm{CH} 4 \mathrm{POLYMER} \mathrm{\&}$ CEMENT CASO4 AGNO3 HNO3 HNO2 NO3- $\mathrm{H}+\mathrm{E}-\mathrm{AG}+\mathrm{AG}++\&$ $\mathrm{H} 2 \mathrm{O} 2$ AGCL CL- NANO3 NH3 CU OH-RAD FRACS $=0.010 .01$ \& $0.011111111111111111111 \&$ $1111111111111110.010 .0111 \&$ 111111111111111

FRAC STREAM $=$ PHOTO-IN SUBSTREAM $=$ CISOLID COMPS=C-S SIO2-S \& AL,2O3-S CEMENT FRACS $=0.01111$

BLOCK PB-DECON SEP

FRAC STREAM $=$ LEAD-OUT SUBSTREAM=MIXED COMPS $=$ H2O PB FRACS $=\&$ 01

FRAC STREAM=LEAD-OUT SUBSTREAM=CISOLID COMPS=C-S SIO2-S \& AL2O3-S CEMENT FRACS $=0000$

BLOCK PB-SPLIT SEP

FRAC STREAM $=$ PBBRICKS SUBSTREAM $=$ MIXED COMPS $=$ H2 $S$ FRACS $=$ \& 0.810 .81

MASS-FLOW STREAM=PBBRICKS SUBSTREAM=MIXED COMPS=PB FLOWS $=$ \& 21

FRAC STREAM=PBBRICKS SUBSTREAM=CISOLID COMPS=C-S SIO2-S \& AL2O3-S FRACS $=0.810 .810 .81$

;Perry's Handbook p. 20-33 shows moisture contents of below $1 \%$ from dryers.

BLOCK WATERSEP SEP

DESCRIPTION "This separates water in polymer stab. subsystem"

FRAC STREAM $=$ PLYSTBIN SUBSTREAM $=$ MIXED COMPS $=\mathrm{C} \mathrm{C-S} \mathrm{H} 2 \mathrm{O} 2$ \& N2 S CL2 F2 NO H2O SIO2 SIO2-S AL2O3 AL2O3-S FE AS \&

BA CD CR PB HG SE AG NAOH NACL CO2 $\mathrm{CO} O 3 \mathrm{HCL}$ \& FECL3 H2SO4 NA2SO4 CH4 POLYMER CEMENT CASO4 AGNO3 \& HNO3 HNO2 NO3- $\mathrm{H}+\mathrm{E}-\mathrm{AG}+\mathrm{AG}++\mathrm{H} 2 \mathrm{O} 2 \mathrm{AGCL} \mathrm{CL}-\mathrm{NANO} 3 \&$ NH3 ERACS $=11110111011111111 \&$

$111111110001111011110 \&$ 10000001010

FRAC STREAM $=$ PLYSTBN SUBSTREAM $=$ CISOLID COMPS $=$ C-S SIO2-S \& AL2O3-S CEMENT FRACS=1 111

BLOCK DRYER HEATER

DESCRIPTION "This models the dryer for the polymer stab. sys." PARAM TEMP $=200$ PRES $=1$

BLOCK HETRHEPA HEATER 
CONV 1 MOXED NO3- 0.99

CONV 2 MIXED CL- 1

BLOCK MEO-ANOD RSTOIC

DESCRIPTION "MEO reactor"

PARAM TEMP $=80<C>$ PRES $=35<$ PSI $>$ SERIES $=$ NO TOL $=0.000001$

STOIC 1 CISOLID C-S - $1 /$ MIXED H2O $-2 / \mathrm{CO}_{1} 1 / \mathrm{H}+\&$

$4 / \mathrm{E}-4$

STOIC 2 MIXED H2 -1/ H $+2 / \mathrm{E}-2$

STOIC 3 MIXED CL2 -1/H+ -2/E- $-2 / \mathrm{HCL} 2$

STOIC 4 MLXED S -2 / H2O -2 / O2 -3/ H2SO4 2

STOIC 5 MIXED N2 -2/ H2O -2/O2 -5/ HNO3 4

CONV 1 CISOLID C-S 0.99

CONV 2 MIXED H2 1

CONV 3 MIXED CL2 1

CONV 4 MIXED S 1

CONV 5 MIXED N2 1

BLOCK MEO-CATH RSTOIC

DESCRIPTION "MEO reactor"

PARAM TEMP $=80<C>$ PRES $=35<P S \perp>$ SERIES $=\mathrm{YES} \mathrm{TOL}=0.000001$

STOIC 1 MLXED HNO3 -1 / H+ -2 / E- -2 / H2O $1 /$ \&

HNO2 1

STOIC 2 MIXED AGNO3 -1 / HCL -1 / AGCL 1 / HNO3 1

CONV 1 MIXED HNO3 1

CONV 2 MIXED HCL 0.99

BLOCK NEUTRAL RSTOIC

PARAM TEMP $=68$ PRES $=1$

STOIC 1 MIXED NAOH - 1 / HCL $-1 /$ NACL $1 /$ H2O 1

STOIC 2 MDXED NAOH - 1 / $\mathrm{HNO} 3$ - 1 / NANO3 $1 /$ H2O 1

STOIC 3 MIXED CL $-1 /$ NAOH $-1 / \mathrm{H}+-1 /$ NACL $1 /$ \&

$\mathrm{H} 2 \mathrm{O} 1$

STOIC 4 MIXED NO3- -1/ H+ -1/ NAOH -1/NANO3 $1 /$ \& $\mathrm{H} 2 \mathrm{O} 1$

CONV 1 MIXED HCL 0.99

CONV 2 MIXED HNO3 0.99

CONV 3 MIXED CL- 0.99

CONV 4 MIXED NO3- 1

;scrubbing efficiency of 0.99 assumed for now

BLOCK NEUTRAL 3 RSTOIC

DESCRIPTTON "neutralizes $\mathrm{HCl}$ in offgas stream"

PARAM TEMP $=100$ PRES $=1$

STOIC 1 MIXED HCL - 1 / NAOH -1 / NACL 1 / H2O 1

STOIC 2 MLXED CL2 -1/ NAOH -2/ NACL 2/ H2O 1/ \& 020.5

STOIC 3 MLXED H2SO4 -1 / NAOH -2 / NA2SO4 1 / $\mathrm{H}_{2} \mathrm{O} 2$

STOIC 4 MIXED SO2 -1 / NAOH -2 / O2 -0.5/NA2SO4 1 \& $/ \mathrm{H} 2 \mathrm{O} 1$

STOIC 5 MLXED HNO2 -1/ NAOH -1/ $02-0.5 / \mathrm{H} 2 \mathrm{O} 1 /$ \&

NANO3 1

CONV 1 MDXED HCL 0.99

CONV 2 MIXED CL 20.99

CONV 3 MIXED H2SO4 0.99

CONV 4 MIXED SO2 0.99

CONV 5 MIXED HNO2 1

BLOCK NOX-CONV RSTOIC

PARAM TEMP $=68$ PRES $=1$

STOIC 1 MIXED NO $-6 / \mathrm{NH} 3-4 / \mathrm{N} 25 / \mathrm{H} 2 \mathrm{O} 6$

CONV 1 MIXED NO 0.99 


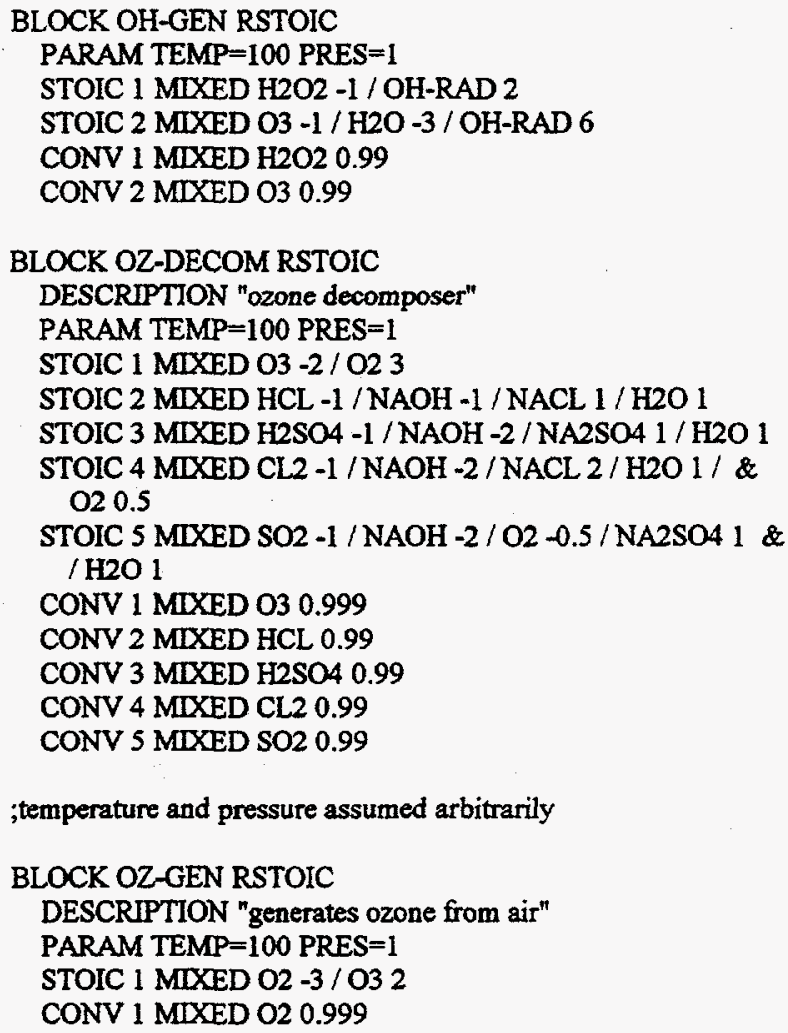

;Destruction efficiency of $96 \%$ assumed (p. 2-78 of "Description of Recommended ;Non-Thermal Mixed Waste Treatment Technologies Version 1.0," Emile A. Bernard ;temperature and pressure assumed by BWBrown

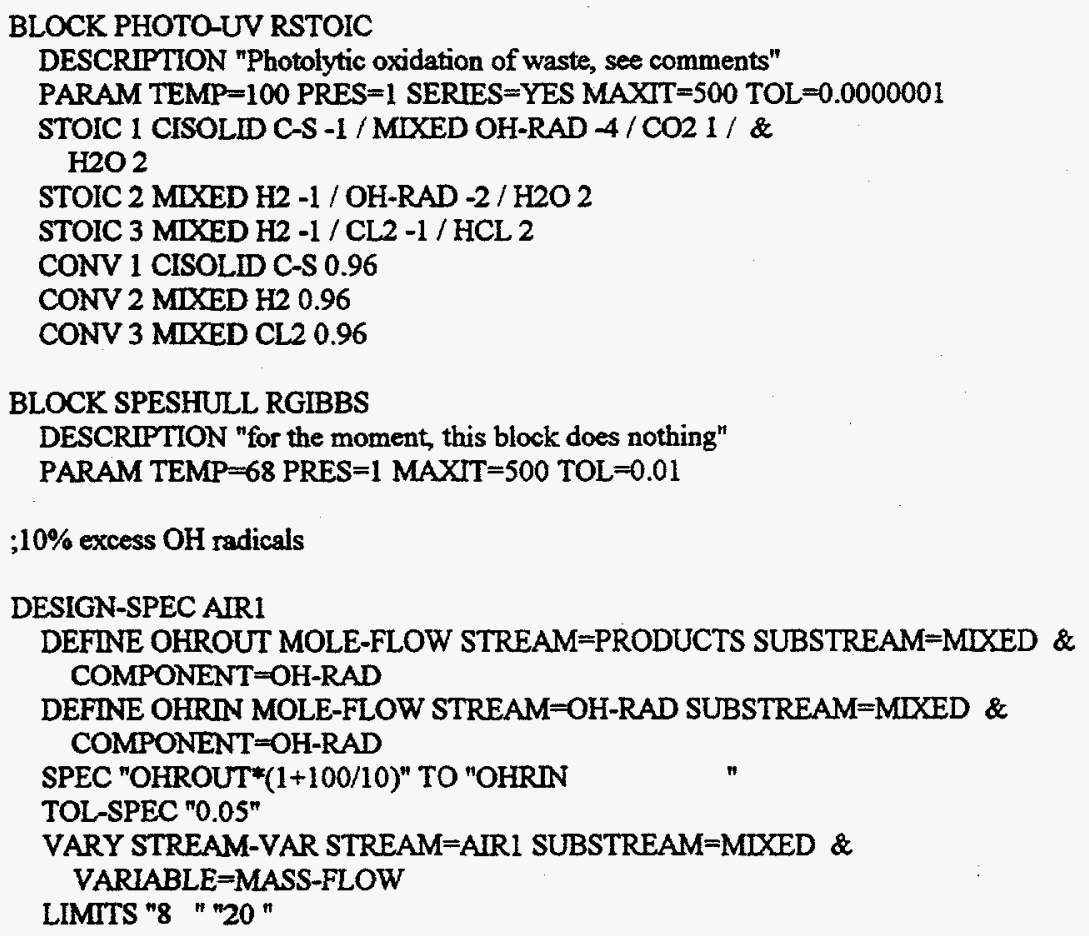




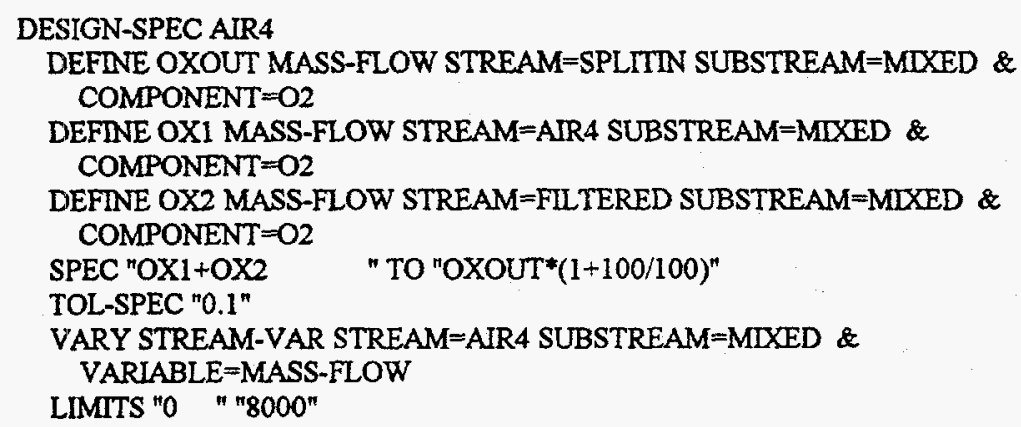

;Perry's Handbook p. 20-33 has moisture contents about $0.5 \%$ from dryers ;review meeting of $2 / 8 / 96$ : moisture content to $5 \%$

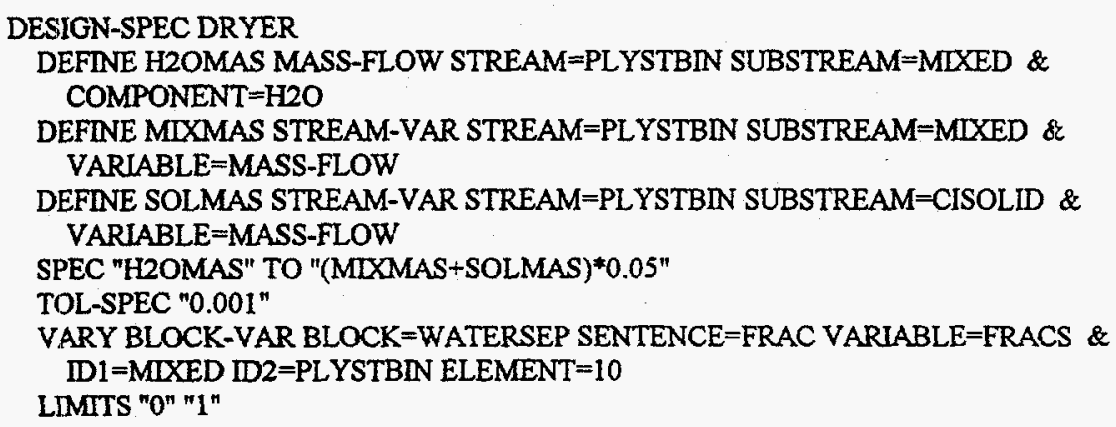

; conversation with Daryoush Bahar 3/6/96 - dry basis used for grout calculation ;because with System 3, too much water exists, which results in too much grout ;being calculated

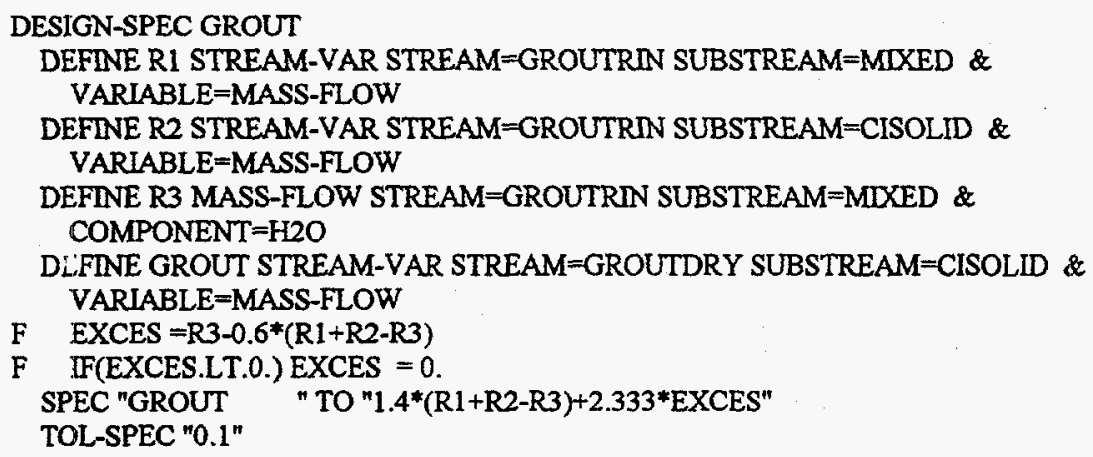


VARY STREAM-VAR STREAM=GROUTDRY SUBSTREAM=CISOLID \& VARIABLE $=$ MASS-FLOW

LIMITS "0.1" "6000"

DESIGN-SPEC GROUTH2O

DEFINE R1 STREAM-VAR STREAM=GROUTRIN SUBSTREAM=MIXED \& VARIABLE $=$ MASS-FLOW

DEFINE R2 STREAM-VAR STREAM=GROUTRIN SUBSTREAM=CISOLID \& VARIABLE $=$ MASS-FLOW

DEFINE R3 MASS-FLOW STREAM=GROUTRIN SUBSTREAM=MIXED \& COMPONENT $=\mathrm{H} 2 \mathrm{O}$

DEFINE H2OFLO STREAM-VAR STREAM=GROUTH2O SUBSTREAM=MIXED \& VARIABLE $=$ MASS-FLOW

F $\quad \mathrm{EXCES}=\mathrm{R3}-0.6 *(\mathrm{R} 1+\mathrm{R} 2-\mathrm{R} 3)$

F IF(EXCES.GT.0.) EXCES $=0.0$

C SETS EXCESS WATER TO O IF WATER IS ACTUALLY EXCESS

C WATER REQUIRED, IF ANY, IS RETURNED AS A NEGATTVE NUMBER

SPEC "H2OFLO" TO "-EXCES"

TOL-SPEC "0.01"

VARY STREAM-VAR STREAM=GROUTH2O SUBSTREAM=MLXED \& VARIABLE=MASS-FLOW

LIMITS "0" "2000"

DESIGN-SPEC HCL-DN

DEFINE HCLOUT MOLE-FLOW STREAM=PPT-OUT SUBSTREAM=MIXED \& COMPONENT $=\mathrm{HCL}$

DEFINE HCLIN MOLE-FLOW STREAM=HCL-IN SUBSTREAM=MIXED \& COMPONENT $=\mathrm{HCL}$

DEFINE CLOUT MOLE-FLOW STREAM=PPT-OUT SUBSTREAM=MIXED \& COMPONENT $=\mathrm{CL}$ -

SPEC "HCLIN "TO "HCLOUT* $(1+100 / 1) "$

TOL-SPEC "0.01"

VARY STREAM-VAR STREAM=HCL-IN SUBSTREAM=MDXED \& VARIABLE $=$ MOLE-FLOW

LIMITS "0.00001" "60"

;This design spec is merely to provide enough excess

; HNO3 to allow the reaction to proceed ( $1 \%$ excess).

DESIGN-SPEC HNO3-IN1

DEFINE HNO3OT MASS-FLOW STREAM=AGRECOVR SUBSTREAM $=$ MIXED \& COMPONENT $=$ HNO3

CEFINE NO3DN1 MASS-FLOW STREAM=NITRIC-2 SUBSTREAM=MIXED \& COMPONENT $=$ HNO3

DEFINE NO3IN2 MASS-FLOW STREAM=HNO3-IN1 SUBSTREAM=MIXED \& COMPONENT $=$ HNO3

SPEC "NO3NI+NO3N2 "TO "HNO3OT* $(1+100 / 1) "$

TOL-SPEC "0.05"

VARY STREAM-VAR STREAM=HNO3-IN1 SUBSTREAM=MIXED \& VARIABLE $=$ MASS-FLOW

LIMITS "0.00001" "90"

DESIGN-SPEC NAOH-3

DEFINE NAOHIN MOLE-FLOW STREAM $=$ NAOH-3 SUBSTREAM=MIXED \& COMPONENT $=\mathrm{NAOH}$

DEFINE NAOHOT MOLE-FLOW STREAM=SPLITN SUBSTREAM=MOXED \& COMPONENT $=\mathrm{NAOH}$

SPEC "NAOHIN" TO "NAOHOT* $(1+100 / 1)$ "

TOL-SPEC "0.001"

VARY STREAM-VAR STREAM=NAOH-3 SUBSTREAM=MEXD \& VARIABLE=MASS-FLOW

LIMITS " 5.0 " "10." 


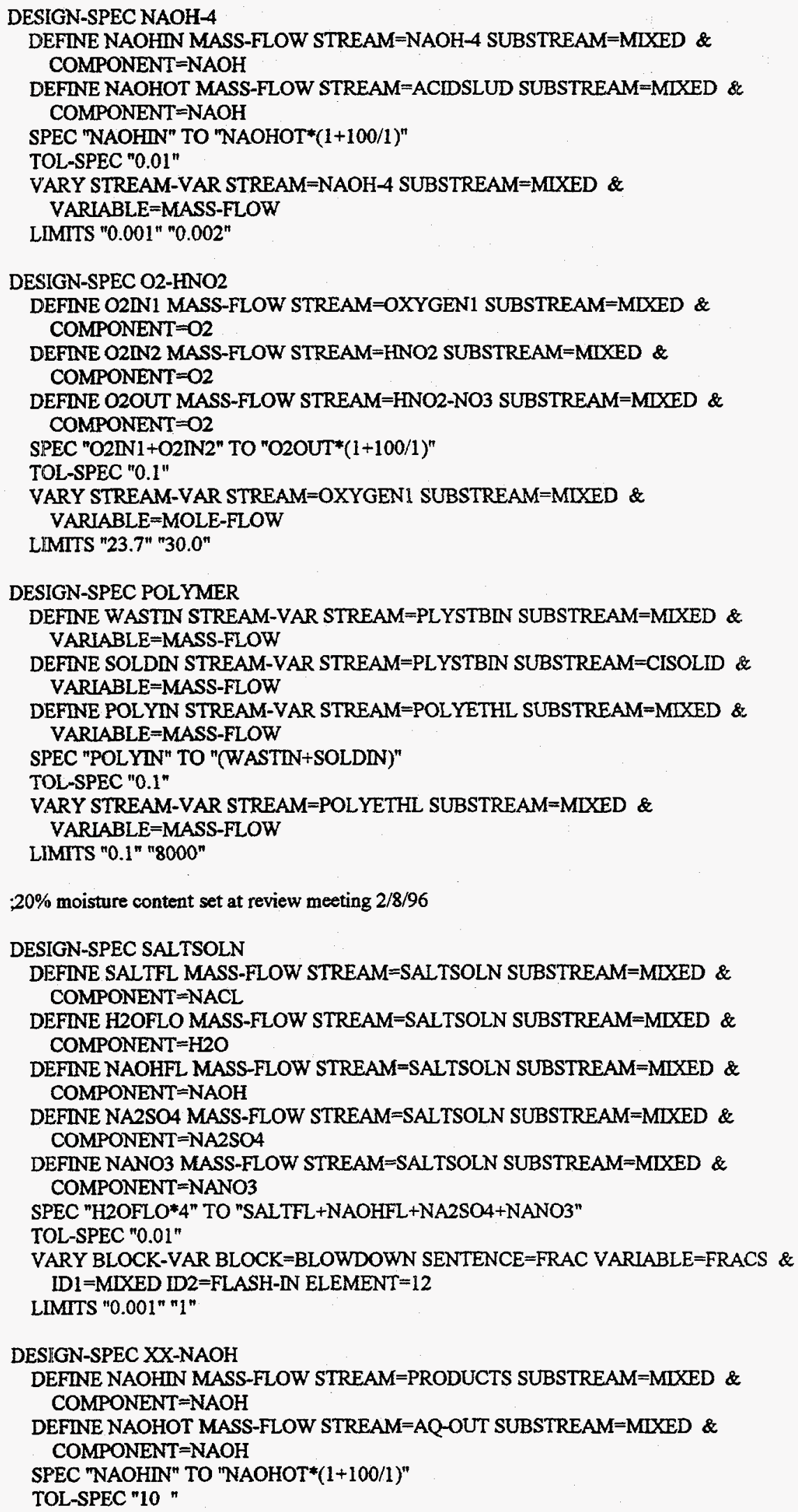




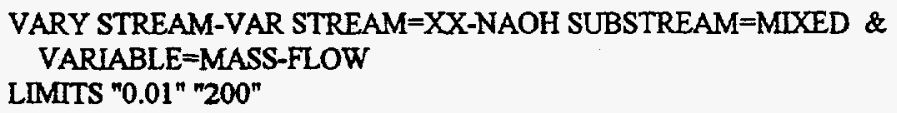

FORTRAN AGRECOVR

DEFINE AGCLIN MOLE-FLOW STREAM=NITRIC-2 SUBSTREAM=MIXED \& COMPONENT $=$ AGCL

DEFINE NAOHIN MOLE-FLOW STREAM=NAOH-1 SUBSTREAM=MLXED \& COMPONENT $=$ NAOH

DEFINE H2O2IN MOLE-FLOW STREAM $=\mathrm{H} 2 \mathrm{O} 2-\mathrm{IN}$ SUBSTREAM $=$ MIXED \& COMPONENT $=\mathrm{H} 2 \mathrm{O} 2$

DEFINE MASSNA MASS-FLOW STREAM=NAOH-1 SUBSTREAM=MTXED \& COMPONENT $=$ NAOH

DEFINE MASSH2 MASS-FLOW STREAM=NAOH-1 SUBSTREAM=MIXED \& COMPONENT $=\mathrm{H} 2 \mathrm{O}$

F $\quad$ NAOHIN $=$ AGCLIN

F H2O2IN = AGCLIN

F $\quad$ MASSH $2=$ MASSNA

READ-VARS AGCLIN MASSNA

WRITE-VARS NAOHN H2O2IN MASSH2

FORTRAN H2O2-[N2

DEFINE O3NN MOLE-FLOW STREAM=PHOTO-IN SUBSTREAM=MLXED \& COMPONENT $=03$

DEFDN H2O2IN STREAM-VAR STREAM=H2O2-IN2 SUBSTREAM=MIXED \& VARIABLE $=$ MOLE-FLOW

F $\mathrm{H} 2 \mathrm{O} 2 \mathrm{~N}=\mathrm{O} 3 \mathrm{~N}$

READ-VARS O3IN

WRITE-VARS H2O2IN

FORTRAN HNO3-IN2

DEFDNE HPIN MOLE-FLOW STREAM=MEO1-OUT SUBSTREAM=MIXED \& COMPONENT $=\mathrm{H}+$

DEFINE HNO3IN STREAM-VAR STREAM=HNO3-IN2 SUBSTREAM=MIXED \& VARIABLE=MOLE-FLOW

DEFINE HNO3R1 MOLE-FLOW STREAM=NITRIC-1 SUBSTREAM=MIXED \& 
COMPONENT $=$ HNO3

DEFINE HNO3R2 MOLE-FLOW STREAM=AGRECYC SUBSTREAM=MIXED \& COMPONENT $=$ HNO3

F $\quad$ HNO3IN $=$ HPIN $/ 2-$ HNO3R1 - HNO3R2

F IF (HNO3N.LT . O.0) HNO3N $=0$.

READ-VARS HPIN HNO3R1 HNO3R2

WRITE-VARS HNO3NN

FORTRAN X-NH3

DEFINE NOXIN MOLE-FLOW STREAM=X-NOX SUBSTREAM=MIXED \& COMPONENT $=$ NO

DEFINE XINH3IN STREAM-VAR STREAM=X-NH3 SUBSTREAM=MIXED \& VARIABLE $=$ MOLE-FLOW

F $\quad \mathrm{XNH} 3 \mathrm{NN}=\mathrm{NOXIN} * 2 / 3$

READ-VARS NOXIN

WRITE-VARS XNH3IN

CONV-OPTIONS

PARAM TEAR-METHOD $=$ BROYDEN TOL $=0.00111$

WEGSTEN $M A X I T=9999$

SECANT STEP-SIZE $=0.01 \mathrm{MAX}-\mathrm{STEP}-\mathrm{SIZ}=1$ BRACKET $=$ NO

BROYDEN MAXIT $=999$

STREAM-REPOR MOLEFLOW MASSFLOW 


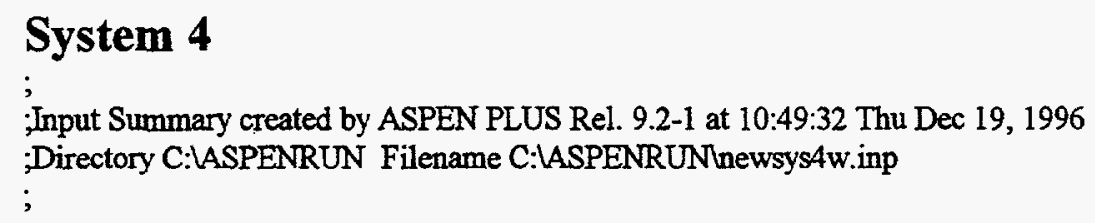


$\mathrm{O} 3 \mathrm{O} 3 \mathrm{O} 3 /$

$\mathrm{H}_{2} \mathrm{O} 2 \mathrm{H}_{2} \mathrm{O} 2 \mathrm{H}_{2} \mathrm{O} 2 /$

HCL HCL HCL /

FECL3 FECL3 FECL3 /

$\mathrm{H}_{2} \mathrm{SO}_{4} \mathrm{H}_{2} \mathrm{SO}_{4} \mathrm{H}_{2} \mathrm{SO} 4 /$

$\mathrm{NA}_{2} \mathrm{SO} 4 \mathrm{NA}_{2} \mathrm{SO} 4 \mathrm{NA}_{2} \mathrm{SO} 4$

$\mathrm{CH} 4 \mathrm{CH} 4 \mathrm{CH} 4$ I

POLYMER C2H4 POLYMER /

CEMENT CAAL2SIO6 CEMENT /

CASO4 $\mathrm{CASO} 4 \mathrm{CASO} 4 /$

HNO3 HNO3 HNO3 /

NANO3 NANO3 NANO3 $/$

NH3 H3N NH3 /

CU CU CU /

OH-RAD OH OH-RAD

FLOWSHEET APC-SYS

BLOCK GPCR IN=FILTERED AIR4 OUT=GPCR-OUT

BLOCK FILTER IN=AQ-OFGAS VACGAS-1 WSHOFGAS DG-GAS-1 \& DG-GAS-2 OUT=FILTERED METALS

BLOCK NEUTRAL3 IN=NAOH-3 GPCR-OUT OUT=SPLITIN

BLOCK BLOWDOWN IN=SPLITIN OUT=FLASH-IN SALTSOLN

BLOCK FLASH2 $\mathbb{N}=$ FLASH-IN OUT=HEATERIN MOISTURE

BLOCK HETRHEPA IN=HEATERIN OUT=OFFGAS

FLOWSHEET AQUEOUS

BLOCK OZ-GEN IN=AIR1 OUT=OZONE

BLOCK CONCENT8 $\mathbb{N}=$ =SALTSOLN OUT =AQ-RECY2 CONCENT8

BLOCK PHOTOMIX IN=VACLIQ-1 METLRECY 1000 WASHLIQ OZONE \& DG-LIQ-1 DG-LIQ-2 MOISTURE H2O2-IN2 HPWATOUT EXCESH2O \& XX-NAOH XY-EXCES AQ-RECY2 OUT $=X-P H O T N N$

BLOCK AQ-SPLIT IN=AQ-OUT OUT=AQ-OFGAS AQ-SLUDG AQ-RECYC

BLOCK OH-GEN IN=PHOTO-IN OUT=OH-RAD

BLOCK PHOTO-UV IN=OH-RAD OUT=PRODUCTS

BLOCK OZ-DECOM IN=PRODUCTS OUT=AQ-OUT

BLOCK OLSEP IN=X-PHOTIN OUT=PHOTO-IN X-ORGS

FLOWSHEET BULKSOI

BLOCK SOLWASH IN=4000 AQ-WASH OUT=WASHLIQ WSHOFGAS \& TRETSOL WASHORG

BLOCK H2OSEP IN=WARMSOL OUT=EXCESH2O DRYSOL

BLOCK SOLDRYER $\mathbb{N}=$ TRETSOL OUT=WARMSOL

FLOWSHEET GROUT

BLOCK GROUTMIX IN=5000COMP WASHDEBR PBGLOVES OUT=GROUTRIN

BLOCK GROUTER IN=GROUTDRY GROUTRIN GROUTH2O OUT=GROUTOUT

FLOWSHEET HG-AMAL

BLOCK HG-AMALG IN=VAC-HG-1 7000-HG ADDITIVE OUT=HG-OUT

FLOWSHEET METLDCON

BLOCK MET-DCON IN=METLWASH 5000MEIL OUT=METLRECY METALOUT

FLOWSHEET ORGDEST

BLOCK DG-SPLT1 $\mathbb{N}=$ =DG-SCRB 1 OUT=DG-LIQ-1 DG-SLD-1 DG-GAS-1

BLOCK ACID-DG1 IN=HNO3-IN1 20003000 WASHORG VACORG-1 \& HNO3RECY X-ORGS OUT=DG-OUT-1 
BLOCK NEUTRAL1 $\mathbb{N}=$ NAOH-1 NH3-IN-1 NOX OUT=DG-SCRB1

BLOCK HNO3-SEP IN=HNO2HNO3 OUT=HNO3RECY NOX

BLOCK HNO2RECV IN=DG-OUT-1 OXYGENI OUT=HNO2HNO3

\author{
FLOWSHEET PB-RECVR \\ BLOCK PB-DECON IN=PBBRICKS PB-WATER OUT=LEAD-OUT PBSLUDGE \\ BLOCK PB-SPLIT IN=7000-PB OUT=PBBRICKS PBGLOVES
}
FLOWSHEET POLYMER
BLOCK PHOSCERM IN=PLYSTBIN POLYETHL OUT=POLY-OUT
BLOCK PHOS-MIX IN=DG-SLD-1 AQ-SLUDG CONCENT8 METALS \&
DG-SLD-2 VACSOL-1 DRYSOL OUT $=X Y-D R Y I N$
BLOCK DRYER IN=XY-DRYIN OUT $=X Y$-DRYOT
BLOCK WATERSEP IN=XY-DRYOT OUT=PLYSTBIN XY-EXCES
BLOCK POLY-MER IN=XYX-PE 3000POLY OUT=XYX-POLY

FLOWSHEET RES-SLUD

BLOCK VACTRAX1 IN=3000 PBSLUDGE N2SWEEP1 OUT=VACOUT-1

BLOCK HP-WASH IN=H2OWASH 5000OPEN OUT=WASHDEBR HPWATOUT

BLOCK NEUTRAL2 IN=NAOH-2 NH3-IN-2 NOX-2 OUT=DG-SCRB2

BLOCK ACID-DG2 $\mathbb{I N}=$ HNO3-IN2 5000SOFT HNO3REC2 OUT=DG-OUT-2

BLOCK DG-SPLT2 IN=DG-SCRB2 OUT=DG-LIQ-2 DG-SLD-2 DG-GAS-2

BLOCK VACSEP-1 IN=VACOUT-1 XYLEACH1 OUT=VACLIQ-1 VACORG-1 \& VACGAS-1 VAC-HG-1 VACSOL-1

BLOCK HNO2REC2 IN=OXYGEN2 DG-OUT -2 OUT =HNO2HN32

BLOCK HNO3SEP2 $\mathbb{N}=\mathrm{HNO} 2 \mathrm{HN} 32$ OUT $=\mathrm{HNO} 3 \mathrm{REC} 2$ NOX-2

\author{
FLOWSHEET SPECIAL \\ BLOCK SPESHULL $\mathbb{N}=6000$ OUT $=$ SPEC-OUT \\ PROPERTIES SOLIDS \\ PROPERTIES DEAL
}

USER-PROPS DRUSR2 123

PROP-DATA U-2

IN-UNITS ENG PRESSURE=ATM

PROP-LIST TC

PVAL CEMENT 1000

PROP-DATA U-1

IN-UNTSS ENG PRESSURE=ATM

PROP-LIST PLXANT

PVAL CEMENT 10-1000 100

PROP-DATA U-1

IN-UNITS ENG PRESSURE=ATM

PROP-LIST DHVLWT

PVAL CEMENT 100000500

;Tables dated 04/24/96 from Daryoush Bahar, MK, (415) 442-7688

;Glass inerts assumed to be $50 \% \mathrm{SiO} 2$ and $50 \% \mathrm{Al} 2 \mathrm{O} 3$ for all waste streams.

\title{
STREAM 1000
}

SUBSTREAM MIXED TEMP $=68$ PRES $=1$ MASS-FLOW $=60.8$

MASS-FLOW H2 $3613<\mathrm{KG} / \mathrm{HR}>/ \mathrm{O} 2172053<\mathrm{KG} / \mathrm{HR}>/ \mathrm{N} 2$ \& $50211<\mathrm{KG} / \mathrm{HR}>/ \mathrm{H} 2 \mathrm{O} 2456410<\mathrm{KG} / \mathrm{HR}>$ / AS $5647<\mathrm{KG} / \mathrm{HR}>\&$ 
/ BA $2823<\mathrm{KG} / \mathrm{HR}>$ / CD $42352<\mathrm{KG} / \mathrm{HR}>$ / CR \& $56469<\mathrm{KG} / \mathrm{HR}>/$ PB $14117<\mathrm{KG} / \mathrm{HR}>/$ HG $14117<\mathrm{KG} / \mathrm{HR}>/ \&$ SE $2823<\mathrm{KG} / \mathrm{HR}>/$ AG $2823<\mathrm{KG} / \mathrm{HR}>$

;Tables dated 04/24/96 from MK

STREAM 3000

SUBSTREAM MIXED TEMP $=68$ PRES $=1$ MASS-FLOW $=352.12$ MASS-FLOW H2 $268596<\mathrm{KG} / \mathrm{HR}>/ \mathrm{O} 210180<\mathrm{KG} / \mathrm{HR}>/ \mathrm{N} 2$ \& $122<\mathrm{KG} / \mathrm{HR}>$ / $6233<\mathrm{KG} / \mathrm{HR}>$ / CL2 $383185<\mathrm{KG} / \mathrm{HR}>$ / \& $\mathrm{H} 2018588527<\mathrm{KG} / \mathrm{HR}>/ \mathrm{FE} 107084<\mathrm{KG} / \mathrm{HR}>/ \mathrm{AS} \&$ $3383<\mathrm{KG} / \mathrm{HR}>/ \mathrm{BA} 12700<\mathrm{KG} / \mathrm{HR}>/ \mathrm{CD} 158485<\mathrm{KG} / \mathrm{HR}>/$ \& CR $299848<\mathrm{KG} / \mathrm{HR}>$ / PB $9640<\mathrm{KG} / \mathrm{HR}>$ / HG $8445<\mathrm{KG} / \mathrm{HR}>\&$ / SE $1689<\mathrm{KG} / \mathrm{HR}>$ / AG $1693<\mathrm{KG} / \mathrm{HR}>$

SUBSTREAM CISOLID TEMP $=68$ PRES $=1$ MASS-FLOW $=655.08$

MASS-FLOW C-S $1658863<$ KG/HR > / SIO2-S $17644467<\mathrm{KG} / \mathrm{HR}>$ / \& AL2O3-S $17644467<\mathrm{KG} / \mathrm{HR}>$

;tables dated 04/24/96 from MK

STREAM 3000POLY

SUBSTREAM MIXED TEMP $=68$ PRES $=1$ MASS-FLOW $=3.28$

MASS-FLOW CL2 $98888<\mathrm{KG} / \mathrm{HR}>$ / NO $37279<\mathrm{KG} / \mathrm{HR}>$ / SO2 \& $47075<\mathrm{KG} / \mathrm{HR}>$

SUBSTREAM CISOLID TEMP=68 PRES=1 MASS-FLOW=3.32

MASS-FLOW SIO2-S 92796 / AL2O3-S 92796

;tables dated 04/24/96 from MK

STREAM 4000

SUBSTREAM MIXED TEMP $=68$ PRES $=1$ MASS-FLOW $=40.74$ MASS-FLOW H2 $21653<\mathrm{KG} / \mathrm{HR}>$ / CL2 $761580<\mathrm{KG} / \mathrm{HR}>/ \mathrm{H} 2 \mathrm{O} \&$ $1514279<\mathrm{KG} / \mathrm{HR}>$

SUBSTREAM CISOLID TEMP $=68$ PRES $=1$ MASS-FLOW $=287.76$

MASS-FLOW C-S $175090<\mathrm{KG} / \mathrm{HR}>$ / SIO2-S $8027287.5<\mathrm{KG} / \mathrm{HR}>$ / \& AL2O3-S 8027287.5 <KG/HR>

;Data table dated 04/24/96. Supplied by Daryoush Bahar, MK (415) 442-7688

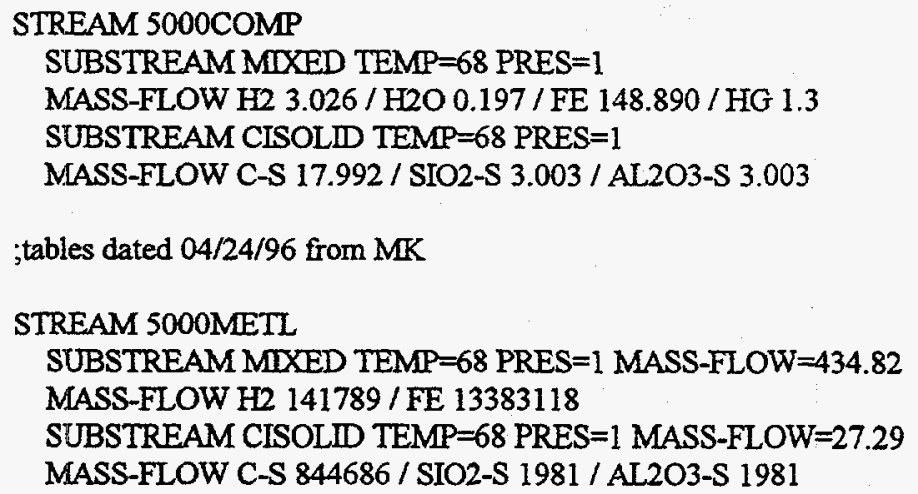

;tables dated 04/24/96 from MK

STREAM 5000METL

SUBSTREAM MIXED TEMP $=68$ PRES $=1$ MASS-FLOW $=434.82$ MASS-FLOW H2 141789 / FE 13383118 SUBSTREAM CISOLID TEMP $=68$ PRES $=1$ MASS-FLOW=27.29 MASS-FLOW C-S 844686 / SIO2-S 1981 / AL2O3-S 1981

;tables dated 04/24/96 from MK

STREAM 5000OPEN

SUBSTREAM MIXED TEMP $=68$ PRES $=1$ MASS-FLOW $=0.40$ 


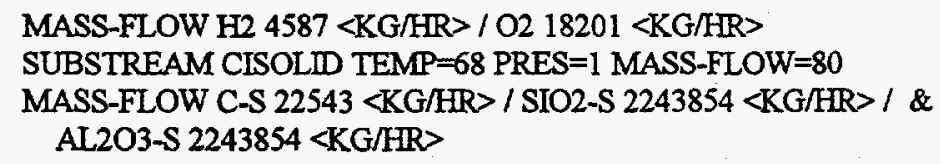

;tables dated 04/24/96 from MK

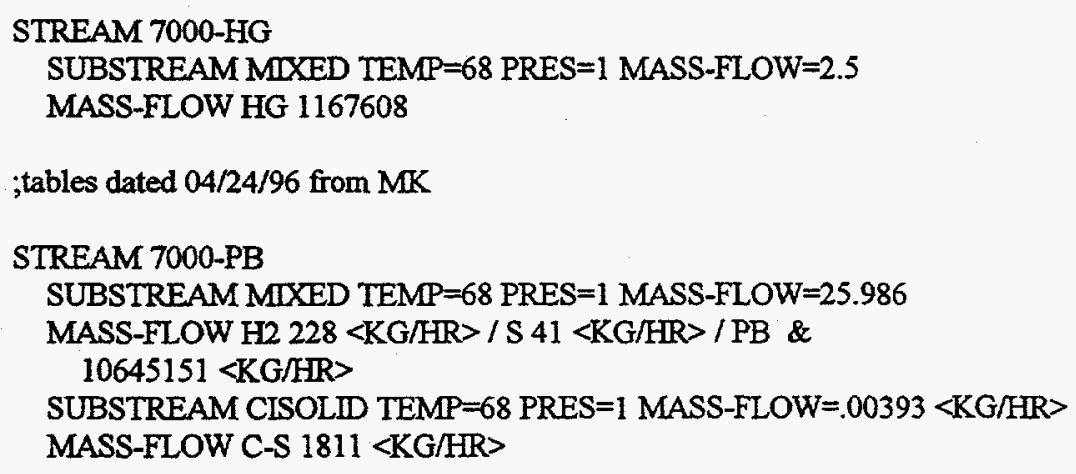




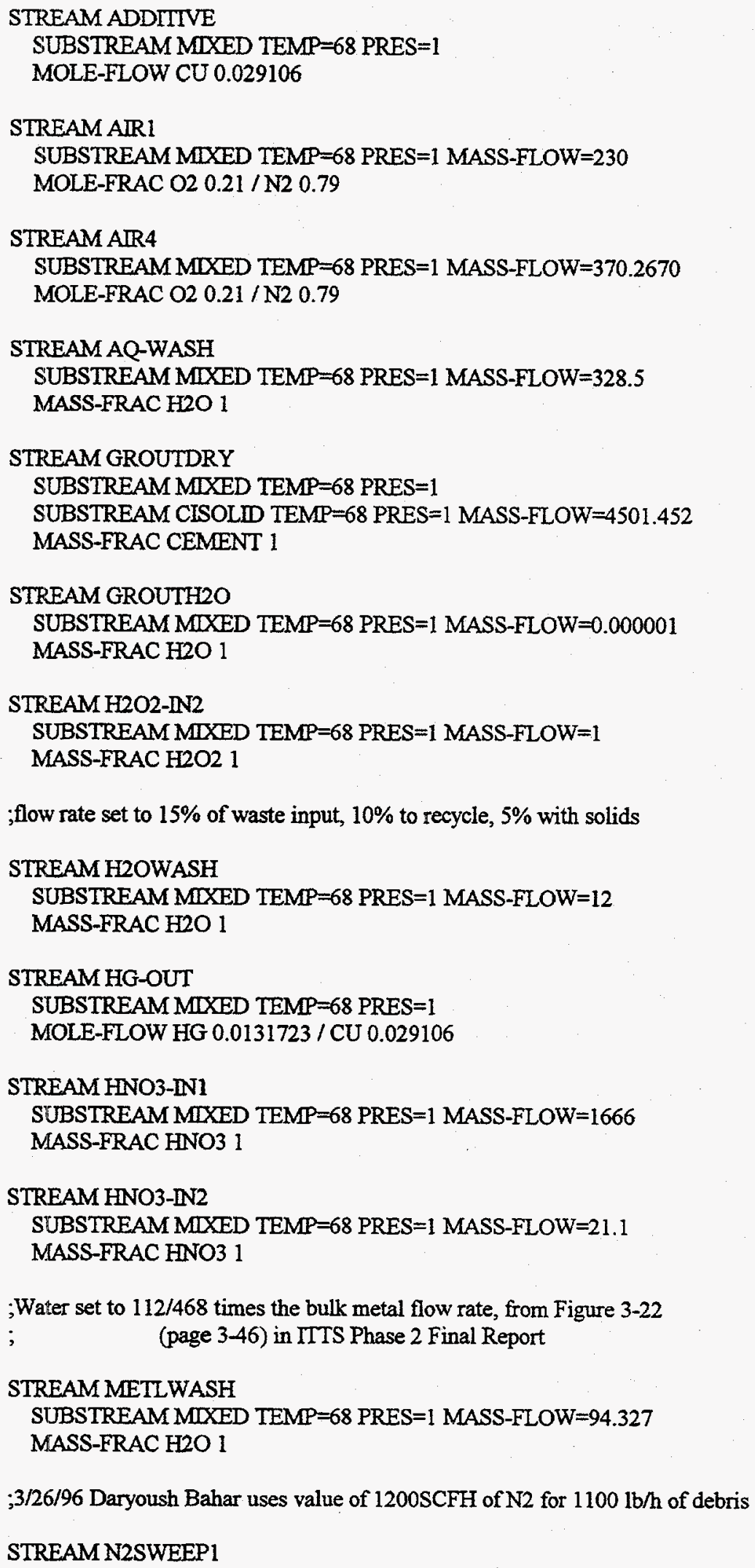


SUBSTREAM MIXED TEMP=68 PRES=1 VOLUME-FLOW=1200 MOLE-FRAC N2 1

;According to Delphi flowsheet D-1002A, 50\% NaOH solution

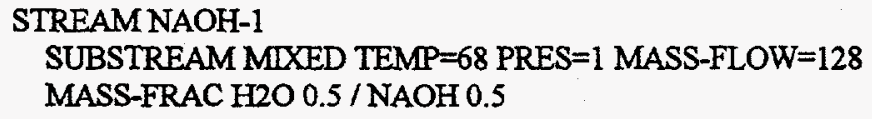

;According to Delphi flowsheet D-1002A, 50\% NaOH solution

STREAM NAOH-2

SUBSTREAM MIXED TEMP=68 PRES=1 MASS-FLOW=73.7

MASS-FRAC H2O 0.5 / NAOH 0.5

STREAM NAOH-3

SUBSTREAM MIXED TEMP=68 PRES=1 MASS-FLOW=34.21764

MASS-FRAC $\mathrm{H} 2 \mathrm{O} 0.5$ / NAOH 0.5

STREAM NH3-IN-1

SUBSTREAM MIXED TEMP=68 PRES=1 MASS-FLOW=290.7029

MASS-FRAC NH3 1

STREAM NH3-IN-2

SUBSTREAM MIXED TEMP $=68$ PRES $=1$ MASS-FLOW $=420$

MASS-FRAC NH3 1

STREAM OXYGEN1

SUBSTREAM MIXED TEMP $=68$ PRES $=1$ MASS-FLOW $=0.0001$

MOLE-FLOW O2 1

STREAM OXYGEN2

SUBSTREAM MIXED TEMP $=68$ PRES $=1$ MASS-FLOW $=0.0001$

MOLE-FLOW O2 1

STREAM PB-WATER

SUBSTREAM MIXED TEMP=68 PRES $=1$ MASS-FLOW $=13$

MASS-FRAC H2O 1

;Polymer assumed to be polyethylene; $\mathrm{C} 2 \mathrm{H} 4$ used as the monomer.

STREAM POLYETHL SUBSTREAM MIXED IEMP=68 PRES=1 MASS-FLOW=113.4696 MASS-FRAC CEMENT I

STREAM PRODUCTS

SUBSTREAM MIXED TEMP=100 PRES=1

MASS-FLOW H2 .688 / O2 4.908 / N2 108.8 / NO 2.738 / \& $\mathrm{H} 2 \mathrm{O} 851.703 / \mathrm{AS} 0.16 / \mathrm{BA} 0.08 / \mathrm{CD} 1.2 / \mathrm{CR} \&$ $1.6 / \mathrm{PB} 0.4 / \mathrm{HG} 0.43 / \mathrm{SE} 0.08 / \mathrm{AG} 0.08 / \&$ NAOH $0.187 /$ NACL $20.7 / 0332.576 /$ HCL $0.13 / \&$ FECL3 0.6

STREAM XX-NAOH

SUBSTREAM MIXED TEMP=68 PRES=1 MASS-FLOW=1

MASS-FRAC $\mathrm{H} 2 \mathrm{O} 0.5 / \mathrm{NAOH} 0.5$ 


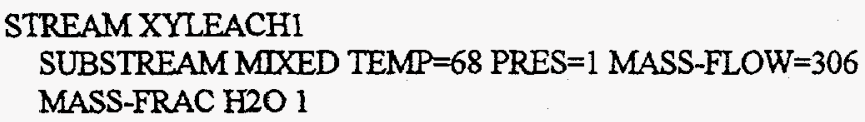

;Polymer assumed to be polyethylene; $\mathrm{C} 2 \mathrm{H} 4$ used as the monomer.

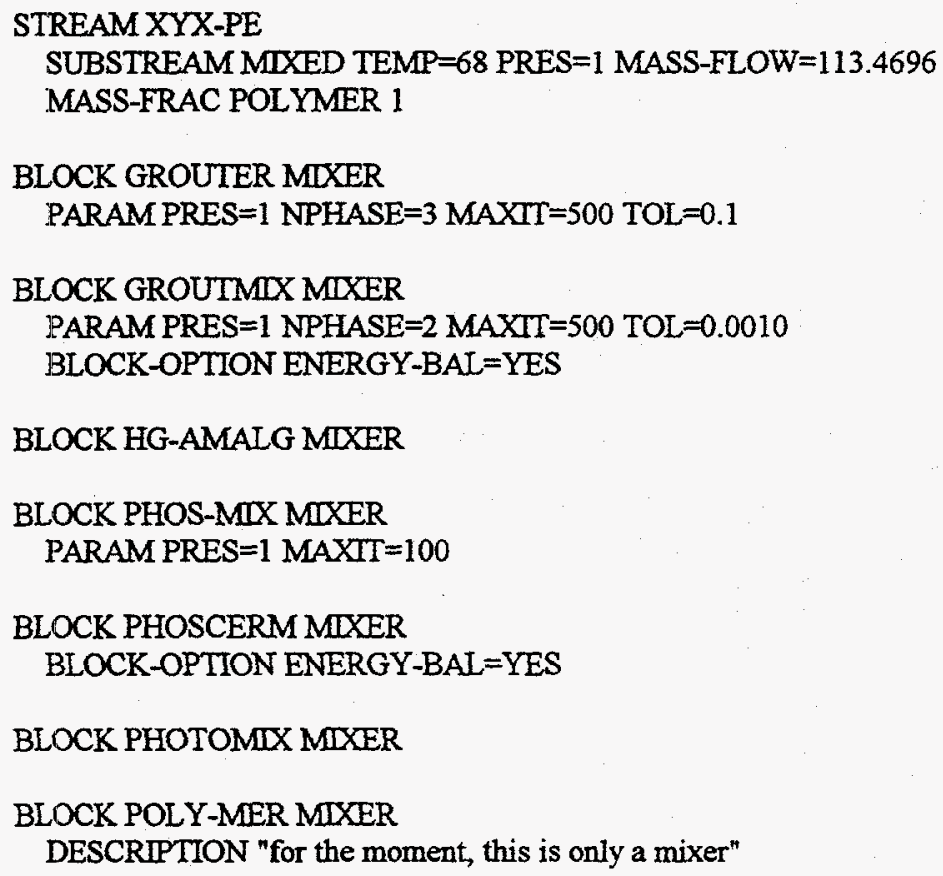

BLDCK SPESHULL MIXER

BLOCK AQ-SPLTT SEP

FRAC STREAM=AQ-OFGAS SUBSTREAM=MIXED COMPS=H2 O2 N2 Cl2 \& F2 NO SO2 $\mathrm{CO} 2 \mathrm{CO} \mathrm{O} 3 \mathrm{CH} 4 \mathrm{NH} 3 \mathrm{FRACS}=11111111 \%$ 011110

FRAC STREAM=AQ-OFGAS SUBSTREAM=CISOLID COMPS=C-S SIO2-S \& AL2O3-S CEMENT FRACS $=0000$

FRAC STREAM=AQ-SLUDG SUBSTREAM=MIXED COMPS=C C-S S SO2 \& SIO2 SIO2-S AL2O3 AL2O3-S FE AS BA CD CR PB HG SE \& AG NAOH NACL FECL3 NA2SO4 POLYMER CEMENT CASO4 NANO3\& $\mathrm{NH} 3 \mathrm{CUFRACS}=11101111111111111 \mathrm{\&}$ 111111111101

FRAC STREAM=AQ-SLUDG SUBSTREAM=CISOLID COMPS=C-S SIO2-S \& AL2O3-S CEMENT FRACS $=0.990 .990 .991$

BLOCK BLOWDOWN SEP

FRAC STREAM=FLASH-IN SUBSTREAM=MIXED COMPS=C C-S H2 O2 \& N2 S CL2 F2 NO SO2 H2O SIO2 STO2-S AL2O3 AL2O3-S \& FE AS BA CD CR PB HG SE AG NAOH NACL $\mathrm{CO} 2 \mathrm{CO} O 3$ \& HCL FECL3 H2SO4 NA2SO4 CH4 POLYMER CEMENT CASO4 HNO3 \& NANO3 NH3 CU FRACS $=11111111110.91 \&$

$111111111111001111110 \&$ 10011111

FRAC STREAM=FLASH-IN SUBSTREAM=CISOLID COMPS=C-S STO2-S \& AL2O3-S CEMENT FRACS $=0000$ 
$; \mathrm{Hg}$ retention in solid set to 0.90 to allow some in gas phase

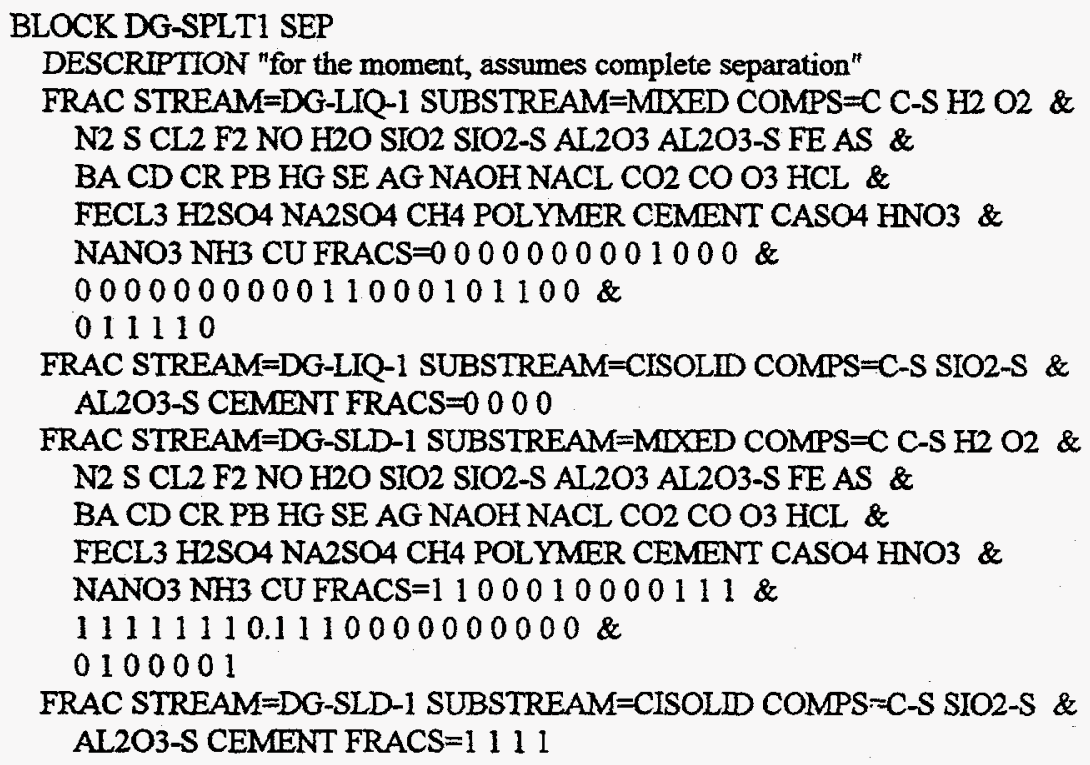

$;$ Hg retention in solid set to 0.95 to allow $5 \%$ in liquid phase

BLOCK DG-SPLT2 SEP

DESCRIPTION "for the moment, assumes complete separation"

FRAC STREAM=DG-LIQ-2 SUBSTREAM=MIXED COMPS=C C-S H2 O2 \& N2 S CL2 F2 NO H2O SIO2 SIO2-S AL2O3 AL2O3-S FE AS \& BA CD CR PB HG SE AG NAOH NACL CO2 CO O3 HCL \& FECL3 $\mathrm{H} 2 \mathrm{SO} 4 \mathrm{NA} 2 \mathrm{SO} 4 \mathrm{CH} 4$ POLYMER CEMENT CASO4 HNO3 \& NANO3 NH3 CU FRACS $=0000000001000 \&$ $00000000.05001100010110 \&$ 0011110

FRAC STREAM=DG-LIQ-2 SUBSTREAM=CISOLD COMPS=C-S SIO2-S \& AL2O3-S CEMENT FRACS $=0000$

FRAC STREAM=DG-SLD-2 SUBSTREAM=MIXED COMPS=C C-S H2 O2 \& N2 S CL2 F2 NO H2O SIO2 SIO2-S AL2O3 AL2O3-S FE AS \& BA CD CR PB HG SE AG NAOH NACL $\mathrm{CO} 2 \mathrm{CO} O 3 \mathrm{HCL}$ \& FECL3 H2SO4 NA2SO4 CH4 POLYMER CEMENT CASO4 HNO3 \& NANO3 NH3 CU FRACS $=1101010000111$ \& 11111110.95110000000000 \& 01000001

FRAC STREAM=DG-SLD-2 SUBSTREAM=CISOLID COMPS=C-S SIO2-S \& AL2O3-S CEMENT FRACS=1 111

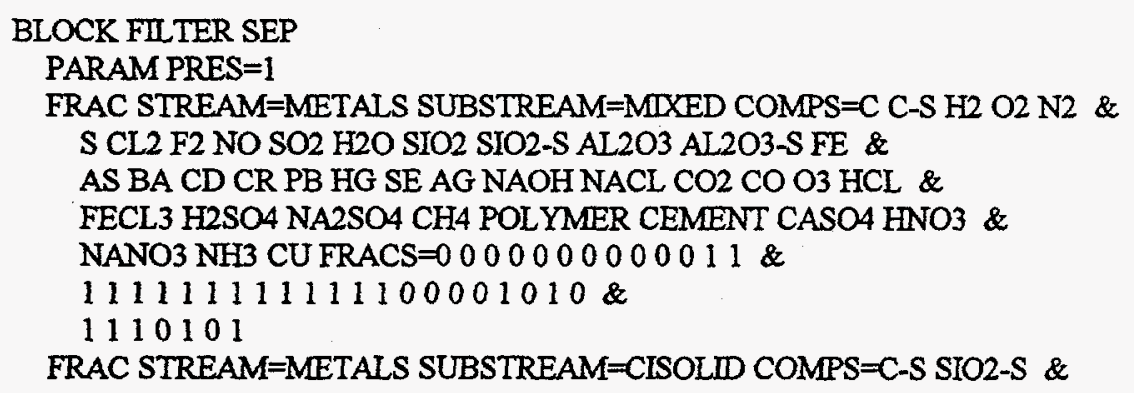




\section{AL2O3-S CEMENT FRACS=1 111}

;This separates the water driven off by the soil dryer after soil washing

BLOCK H2OSEP SEP

DESCRIPTION "separates remaining H2O from washed soil"

FRAC STREAM $=$ EXCESH 20 SUBSTREAM $=$ MIXED COMPS $=C \mathrm{C}-\mathrm{S}$ H2 O2 \& N2 S CL2 F2 NO SO2 H2O SIO2 SIO2-S AL2O3 AL2O3-S \& FE AS BA CD CR PB HG SE AG NAOH NACL $\mathrm{CO} 2 \mathrm{CO} O 3 \&$ $\mathrm{H} 2 \mathrm{O} 2 \mathrm{HCL}$ FECL3 H2SO4 NA2SO4 CH4 POLYMER CEMENT CASO4 \& HNO3 NANO3 NH3 CU OH-RAD FRACS $=000000000 \quad \&$ 001000000000000000000 \& 00000000000000

FRAC STREAM=EXCESH2O SUBSTREAM=CISOLID COMPS=C-S SIO2-S \& AL203-S CEMENT FRACS $=0000$

BLOCK HNO3-SEP SEP

FRAC STREAM $=$ HNO3RECY SUBSTREAM $=$ MIXED COMPS $=\mathrm{C} C-\mathrm{S} \mathrm{H} 2 \mathrm{O} 2$ \& N2 S CL2 F2 NO H2O SIO2 SIO2-S AL2O3 AL2O3-S FE AS \& BA CD CR PB HG SE AG NAOH NACL CO2 CO O3 HCL \& FECL3 H2SO4 NA2SO4 CH4 POLYMER CEMENT CASO4 HNO3 \& NANO 3 NH3 CU FRACS $=0000000000000 \&$ 000000000000000000000 \& 000.99000

FRAC STREAM=HNO3RECY SUBSTREAM $=$ CISOLID COMPS=C-S SIO2-S \& AL2O3-S CEMENT FRACS $=0000$

BLOCK HNO3SEP2 SEP

FRAC STREAM=HNO3REC2 SUBSTREAM=MIXED COMPS $=\mathrm{C} C \mathrm{C}-\mathrm{S} \mathrm{H} 2 \mathrm{O} 2$ \& N2 S CL2 F2 NO H2O SIO2 SIO2-S AL2O3 AL2O3-S FE AS \&

BA CD CR PB HG SE AG NAOH NACL $\mathrm{CO} 2 \mathrm{CO} O 3 \mathrm{HCL} \&$ FECL3 H2SO4 NA2SO4 CH4 POLYMER CEMENT CASO4 HNO3 \& NANO3 NH3 CU FRACS $=0000000000000$ \& $000000000000000000000 \&$ 000.99000

FRAC STREAM=HNO3REC2 SUBSTREAM=CISOLID COMPS=C-S SIO2-S \& AL2O3-S CEMENT FRACS $=0000$

;wash water flow rate set to $15 \%$ of waste input; $10 \%$ to recycle, $5 \%$ to solids

\section{BLOCK HP-WASH SEP}

DESCRIPTION "estimated separation"

FRAC STREAM=WASHDEBR SUBSTREAM=MIXED COMPS=H2 O2 CL2 FE \& FRACS $=0.020 .020 .021$

MASS-FLOW STREAM=WASHDEBR SUBSTREAM=MIXED COMPS=H2O FLOWS $=$ \& 4

FRAC STREAM=WASHDEBR SUBSTREAM=CISOLD COMPS=C-S SIO2-S \& AL2O3-S FRACS $=0.020 .990 .99$

BLOCK MET-DCON SEP

DESCRIPTION "Separates water from metal"

FRAC STREAM=METLRECY SUBSTREAM $=$ MIXED COMPS=C C-S H2 O2 \& N2 S CL2 F2 NO H2O SIO2 SIO2-S AL2O3 AL2O3-S FE AS \& BA CD CR PB HG SE AG NAOH NACL CO2 CO HCL FECL3 \& $\mathrm{H} 2 \mathrm{SO} 4 \mathrm{NA} 2 \mathrm{SO} 4 \mathrm{CH} 4 \mathrm{POLYMER}$ CEMENT FRACS $=001000 \&$ 000100000000000000000 \& 0000000 
FRAC STREAM=METLRECY SUBSTREAM=CISOLID COMPS=C-S SIO2-S \& AL2O3-S CEMENT FRACS $=11110$

BLOCK OLSEP SEP

DESCRIPTION "assumes $99 \%$ separation of organics ( $\mathrm{C}, \mathrm{H}, \mathrm{CHx})^{n}$

FRAC STREAM=PHOTO-IN SUBSTREAM=MIXED COMPS $=\mathrm{C} C \mathrm{C}-\mathrm{S}$ H2 $\mathrm{O} 2$ \& N2 S CL2 F2 NO SO2 H2O SIO2 SIO2-S Al.2O3 AL2O3-S \& FE AS BA BE CD CR PB HG SE AG TA U NAOH NACL \& $\mathrm{CO} 2 \mathrm{CO} \mathrm{O} 3 \mathrm{H} 2 \mathrm{O} 2 \mathrm{HCL}$ FECL 3 H2SO4 NA2SO4 CH4 POLYMER \& CEMENT CASO4 HNO3 NANO 3 NH3 CU OH-RAD FRACS $=0.01 \&$ 0.010 .0111111111111111111 \& $111111111111111110.010 .01 \&$ 1111111

FRAC STREAM=PHOTO-IN SUBSTREAM=CISOLID COMPS=C-S SIO2-S \& AL2O3-S CEMENT FRACS $=0.01111$

BLOCK PB-DECON SEP

FRAC STREAM=LEAD-OUT SUBSTREAM $=$ MIXED COMPS $=$ H2O PB FRACS $=\&$ 01

FRAC STREAM=LEAD-OUT SUBSTREAM=CISOLID COMPS=C-S SIO2-S \& AL2O3-S CEMENT FRACS $=0000$

BLOCK PB-SPLIT SEP

FRAC STREAM $=$ PBBRICKS SUBSTREAM $=\mathrm{MIXED}$ COMPS $=\mathrm{H} 2 \mathrm{~S}$ FRACS $=\&$ 0.810 .81

MASS-FLOW STREAM=PBBRICKS SUBSTREAM=MIXED COMPS=PB FLOWS $=\&$ 21

FRAC STREAM=PBBRICKS SUBSTREAM=CISOLID COMPS=C-S SIO2-S \& AL2O3-S FRACS $=0.810 .810 .81$

;For now, assumes $95 \%$ removal by washing of $\mathrm{C}, \mathrm{H} 2, \mathrm{Cl} 2$

BLOCK SOILWASH SEP

DESCRIPTION "For now, a simple separation (w/o condensers, etc)"

FRAC STREAM=WASHLIQ SUBSTREAM=MIXED COMPS=C C-S H2 O2 \& N2 S CL2 F2 NO SO2 H2O SIO2 SIO2-S AL2O3 AL2O3-S \& FE AS BA BE CD CR PB HG SE AG TA U NAOH NACL \& $\mathrm{CO} 2 \mathrm{CO} \mathrm{O} 3 \mathrm{H} 2 \mathrm{O} 2 \mathrm{HCL}$ FECL3 $\mathrm{H} 2 \mathrm{SO} 4 \mathrm{NA} 2 \mathrm{SO} 4 \mathrm{CH} 4$ POLYMER \& CEMENT CASO4 HNO3 NANO3 NH3 CU OH-RAD FRACS $=000$ \& $000000000000000000000 \&$ 000000000000000000000 \& 0

FRAC STREAM=WASHLIQ SUBSTREAM=CISOLD COMPS=C-S SIO2-S \& AL2O3-S CEMENT FRACS $=0000$

FRAC STREAM=WSHOFGAS SUBSTREAM=MIXED COMPS=C C-S H2 O2 \& N2 S CL2 F2 NO SO2 $\mathrm{H} 20$ SIO2 SIO2-S AL2O3 AL2O3-S \& FE AS BA BE CD CR PB HG SE AG TA U NAOH NACL \& $\mathrm{CO} 2 \mathrm{CO} \mathrm{O} 3 \mathrm{H} 2 \mathrm{O} 2 \mathrm{HCL}$ FECL3 $\mathrm{H} 2 \mathrm{SO} 4 \mathrm{NA} 2 \mathrm{SO} 4 \mathrm{CH} 4 \mathrm{POLYMER} \&$ CEMENT CASO4 HNO3 NANO3 NH3 CU OH-RAD FRACS $=000$ \& $000000000000000000000 \&$ $000000000000000000000 \&$ 0

FRAC STREAM=WSHOFGAS SUBSTREAM=CISOLD COMPS=C-S SIO2-S \& AL2O3-S CEMENT FRACS $=0000$

FRAC STREAM $=$ TRETSOL SUBSTREAM=MIXED COMPS $=\mathrm{C} C \mathrm{C}-\mathrm{S} \mathrm{H} 2 \mathrm{O} 2$ \& N2 S CL2 F2 NO H2O SIO2 SIO2-S AL2O3 AL2O3-S FE AS \& BA CD CR PB HG SE AG NAOH NACL $\mathrm{CO} 2 \mathrm{CO} O 3$ FECL3 \& 
H2SO4 NA2SO4 CH4 POLYMER CEMENT CASO4 HNO3 NANO3 NH3 \& CU FRACS $=0.050 .050 .050 .05010 .0500111 \&$

111111110111100011111 \&

110001

FRAC STREAM=TRETSOL SUBSTREAM=CISOLD COMPS=C-S SIO2-S \& AL2O3-S CEMENT FRACS $=0.05111$

;assumes $50 \%$ desorption of $\mathrm{C}, \mathrm{H} 2 \quad 5 \%$ of $\mathrm{Hg}$ removed by aqueous washing

BLOCK VACSEP-1 SEP

FRAC STREAM $=$ VACLIQ-1 SUBSTREAM=MIXED COMPS $=\mathrm{C}$ C-S H2 O2 \& N2 S CL2 F2 NO SIO2 SIO2-S AL2O3 AL2O3-S FE AS BA \& CD CR PB HG SE AG NAOH NACL CO2 CO O3 HCL FECL 3 \& $\mathrm{H} 2 \mathrm{SO} 4 \mathrm{NA} 2 \mathrm{SO} 4 \mathrm{CH} 4$ POLYMER CEMENT CASO4 HNO3 NANO3 NH3 \& CU FRACS $=00000000000000000 \&$ $000000000010100000000 \&$ 0

MASS-FLOW STREAM=VACLIQ-1 SUBSTREAM $=$ MIXED COMPS=H2O FLOWS $=$ \& 342.5798

FRAC STREAM=VACLIQ-1 SUBSTREAM=CISOLD COMPS=C-S SIO2-S \& AL2O3-S CEMENT FRACS $=0000$

FRAC STREAM=VACORG-1 SUBSTREAM=MIXED COMPS=C C-S H2 O2 \& N2 S CL2 F2 NO H2O SIO2 SIO2-S AL2O3 AL2O3-S FE AS \& BA CD CR PB HG SE AG NAOH NACL CO2 $\mathrm{CO} O 3 \mathrm{HCL} \&$ FECL3 H2SO4 NA2SO4 CH4 POLYMER CEMENT CASO4 HNO3 \& NANO3 NH3 CU FRACS $=000.50000 .50 .5000 \&$ 000000000000000100000 \& 11000000

FRAC STREAM=VACORG-1 SUBSTREAM=CISOLD COMPS=C-S SIO2-S \& AL2O3-S CEMENT FRACS $=0.5000$

FRAC STREAM=VACGAS-1 SUBSTREAM=MIXED COMPS=C C-S H2 O2 \& N2 S CL2 F2 NO H2O SIO2 SIO2-S AL2O3 AL2O3-S FE AS \& BA CD CR PB HG SE AG NAOH NACL $\mathrm{CO} 2 \mathrm{CO} O 3 \mathrm{HCL} \&$ FECL3 $\mathrm{H} 2 \mathrm{SO} 4 \mathrm{NA} 2 \mathrm{SO} 4 \mathrm{CH} 4$ POLYMER CEMENT CASO4 HNO3 \& NANO3 NH3 CU FRACS $=0000100010000$ \& $000000000000101000000 \&$ 000000

FRAC STREAM=VACGAS-1 SUBSTREAM=CISOLID COMPS=C-S SIO2-S \& AL2O3-S CEMENT FRACS $=0000$

FRAC STREAM=VAC-HG-1 SUBSTREAM=MIXED COMPS $=\mathrm{C} C-\mathrm{S} \mathrm{H} 2 \mathrm{O} 2 \&$ N2 S CL2 F2 NO H2O SIO2 SIO2-S AL2O3 AL2O3-S FE AS \& BA CD CR PB HG SE AG NAOH NACL $\mathrm{CO} 2 \mathrm{CO} O 3 \mathrm{HCL} \&$ FECL3 H2SO4 NA2SO4 CH4 POLYMER CEMENT CASO4 HNO3 \& NANO3 NH3 CU FRACS $=0000000000000 \&$ $00000000.95000000000000 \&$ 0000000

FRAC STREAM $=$ VAC-HG-1 SUBSTREAM=CISOLID COMPS=C-S SIO2-S \& AL2O3-S CEMENT FRACS $=0000$

BLOCK WATERSEP SEP

FRAC STREAM $=$ PLYSTBIN SUBSTREAM $=$ MIXED COMPS $=\mathrm{C} C-\mathrm{S}$ H2 $\mathrm{O} 2 \&$ N2 S CL2 F2 NO SO2 $\mathrm{H} 2 \mathrm{O}$ SIO2 SIO2-S AL2O3 AL2O3-S \& FE AS BA BE CD CR PB HG SE AG TA U NAOH NACL \& $\mathrm{CO} 2 \mathrm{CO} O 3 \mathrm{H} 2 \mathrm{O} 2 \mathrm{HCL}$ FECL3 H2SO4 NA2SO4 CH4 POLYMER \& CEMENT CASO4 HNO3 NANO3 NH3 CU OH-RAD FRACS $=111 \&$ $111110011111111111111 \%$ $111111111111111111111 \&$ 
1

FRAC STREAM $=$ PLYSTBIN SUBSTREAM $=$ CISOLD COMPS=C-S SIO2-S \& AL2O3-S CEMENT FRACS=1111

;8/21/96 - dryer added to System 4 after discussing with Julia Vetromile

BLOCK DRYER HEATER

DESCRIPTION "This models the dryer for the polymer stab. sys."

PARAM TEMP=200 PRES=1

BLOCK HETRHEPA HEATER

DESCRIPTION "heater to raise temperature for HEPA filters"

PARAM TEMP=300 PRES=1

BLOCK SOLDRYER HEATER

PARAM TEMP $=200$ PRES $=1$

BLOCK CONCENT8 FLASH2

PARAM TEMP=150 PRES $=1$

BLOCK FLASH2 FLASH2

PARAM TEMP=100 PRES $=1$

;Acid digestion, uses $\mathrm{HNO} 3$ to oxidize $\mathrm{C}$ and $\mathrm{H} 2$

;H3PO4 does not participate in the reaction stoichiometry

BLOCK ACID-DGI RSTOIC

DESCRIPTION "Acid digestion"

PARAM TEMP $=200<C>$ PRES $=1$

STOIC 1 CISOLID C-S -3 / MIXED HNO3 $-4 / \mathrm{CO}_{3} 3$ / NO \& 4 / $\mathrm{H} 2 \mathrm{O} 2$

STOIC 2 MIXED H2 -3/ HNO3 $-2 / \mathrm{H} 2 \mathrm{O} 4 / \mathrm{NO} 2$

STOIC 3 MIXED CL $2-0.5 / \mathrm{H} 2-0.5 / \mathrm{HCL} 1$

STOIC 4 MIXED S -1 / H2 -1/ O2 -2/ H2SO4 1

STOIC 5 MIXED CH4 -3/ HNO3 -8 / NO 8 / $\mathrm{CO} 3 /$ \&

$\mathrm{H} 2 \mathrm{O} 10$

CONV 1 CISOLID C-S 0.95

CONV 2 MIXED H2 0.95

CONV 3 MIXED CL2 0.95

CONV 4 MIXED $\$ 0.95$

CONV 5 MIXED CH4 0.95

;Acid digestion, uses $\mathrm{HNO} 3$ to oxidize $\mathrm{C}$ and $\mathrm{H} 2$

;H3PO4 does not participate in the reaction stoichiometry

BLOCK ACD-DG2 RSTOIC

DESCRIPTION "Acid digestion"

PARAM TEMP $=200<C>$ PRES $=1$

STOIC 1 CISOLD C-S - 3 / MAXED HNO3 4 / $\mathrm{CO}_{3} 3$ / NO \& $4 / \mathrm{H} 2 \mathrm{O} 2$

STOIC 2 MIXED H2 -1.5/ HNO3 -1/ $\mathrm{H}_{2} \mathrm{O} 2 / \mathrm{NO} 1$

STOIC 3 MIXED CL $2-0.5 / \mathrm{H} 2-0.5 / \mathrm{HCL} 1$

STOIC 4 MTXED S - / / H2 - - / O2 -2 / H2SO4 1

CONV 1 CISOLD C-S 0.95

CONV 2 MIXED H2 0.95

CONV 3 MIXED CL2 0.95

CONV 4 MIXED S 0.95 
;Data obtained from "Description of Recommended Non-Thermal.."pp. 6-43 to 6-45 ;Although room temperature operation is listed on p. $6-43,1000 \mathrm{C}$ is listed ;on page $6-45$, and the higher temperature is more believable :DRE of 0.999 listed for TCE and $0.90-0.95$ for PCE $\rightarrow$ use 0.95 for all waste ;assume $1 \mathrm{~atm}$ pressure

BLOCK GPCR RSTOIC

DESCRIPTION "Gas Phase Corona Reactor, see comments"

PARAM PRES $=1$ DUTY $=20000$

STOIC 1 MIXED H2 -2 / O2 -1/ $\mathrm{H} 2 \mathrm{O} 2$

STOIC 2 CISOLID C-S -1/MIXED O2 -1/ CO2 1

STOIC 3 MIXED H2 -1/CL2 -1/ HCL 2

STOIC 4 MIXED CO $-1 / \mathrm{O} 2-0.5 / \mathrm{CO} 21$

STOIC 5 MIXED CH4 -1/O2 -2/ CO2 1/ $\mathrm{H} 2 \mathrm{O} 2$

CONV 1 MIXED H2 0.95

CONV 2 CISOLID C-S 0.95

CONV 3 MIXED CL2 0.95

CONV 4 MIXED CO 0.95

CONV 5 MIXED CH4 0.95

BLOCK HNO2REC2 RSTOIC

PARAM TEMP $=423<\mathrm{K}>\mathrm{PRES}=35<\mathrm{PSI}>$

STOIC 1 MIXED NO $-4 / \mathrm{O} 2-3 / \mathrm{H} 2 \mathrm{O}-2 / \mathrm{HNO} 34$

CONV 1 MIXED NO 0.99

BLOCK HNO2RECV RSTOIC

PARAM TEMP $=423<\mathrm{K}>$ PRES $=35<$ PSI $>$

STOIC 1 MIXED NO - $/$ / O2 -3/ H2O - $/$ / HNO3 4

CONV 1 MIXED NO 0.99

BLOCK NEUTRAL1 RSTOIC

DESCRPTION "neutralizes excess $\mathrm{HCl}$, NO"

PARAM TEMP=100 PRES=1 SERIES=YES

STOIC 1 MIXED NAOH - $1 /$ HCL - $1 /$ NACL $1 /$ H2O 1

STOIC 2 MIXED H2SO4 - $1 / \mathrm{NAOH}-2$ / NA2SO4 $1 /$ H2O 2

STOIC 3 MIXED CL2 -1 / NAOH -2 / NACL 2 / H2O 1 / \&

020.5

STOIC 4 MIXED NO - $6 /$ NH3 $-4 /$ N2 5/ H2O 6

STOIC 5 MIXED HNO3 - / NAOH -1/ NANO3 $1 /$ H2O 1

CONV 1 MIXED HCL 0.99

CONV 2 MIXED H2SO4 0.99

CONV 3 MIXED CL2 0.99

CONV 4 MIXED NO 0.99

CONV 5 MLXED HNO3 0.99

BLOCK NEUTRAI2 RSTOIC

DESCRIPTION "neutralizes excess $\mathrm{HCl}$ "

PARAM TEMP=100 PRES=1 SERIES=YES

STOIC 1 MIXED NAOH $-1 / \mathrm{HCL}-1 / \mathrm{NACL} 1 / \mathrm{H} 2 \mathrm{O} 1$

STOIC 2 MIXED H2SO4 -1/ NAOH -2/ NA2SO4 $1 /$ H2O 2

STOIC 3 MIXED CL2 -1 / NAOH -2 / NACL $2 /$ H2O $1 /$ \&

020.5

STOIC 4 MIXED NO -3 / NH3 - 2 / N2 2.5 / H2O 3

STOIC 5 MIXED HNO3 - $1 /$ NAOH - $1 /$ NANO3 $1 /$ H2O 1

CONV 1 MIXED HCL 0.99

CONV 2 MIXED H2SO4 0.99 
CONV 3 MIXED CL2 0.99

CONV 4 MIXED NO 0.99

CONV 5 MIXED HNO3 0.99

;scrubbing efficiency of 0.99 assumed for now

BLOCK NEUTRAL3 RSTOIC

DESCRIPTION "neutralizes $\mathrm{HCl}$ in offgas stream"

PARAM TEMP $=100$ PRES $=1$

STOIC 1 MIXED HCL $-1 / \mathrm{NAOH}-1 / \mathrm{NACL} 1 / \mathrm{H} 2 \mathrm{O} 1$

STOIC 2 MIXED CL2 - 1 / NAOH -2 / NACL $2 / \mathrm{H} 2 \mathrm{O} 1 /$ \& $\mathrm{O} 20.5$

STOIC 3 MIXED H2SO4 -1/ NAOH - 2 / NA2SO4 1 / $\mathrm{H}_{2} \mathrm{O} 2$

STOIC 4 MIXED SO2 -1/ NAOH $-2 / 02-0.5 / \mathrm{NA2SO} 1$ \& / $\mathrm{H} 2 \mathrm{O} 1$

CONV 1 MIXED HCL 0.99

CONV 2 MIXED CL2 0.99

CONV 3 MIXED H2SO4 0.99

CONV 4 MIXED SO2 0.99

BLOCK OH-GEN RSTOIC

PARAM TEMP $=100$ PRES $=1$

STOIC 1 MIXED H2O2 -1 / OH-RAD 2

STOIC 2 MIXED O3 - / / H2O -3 / OH-RAD 6

CONV 1 MIXED H2O2 0.99

CONV 2 MIXED 030.99

BLOCK OZ-DECOM RSTOIC

DESCRIPTION "ozone decomposer"

PARAM TEMP $=100$ PRES=1

STOIC 1 MIXED O3 -2/ O2 3

STOIC 2 MIXED HCL -1 / NAOH -1 / NACL $1 /$ H2O 1

STOIC 3 MIXED H2SO4 - 1 / NAOH -2/ NA2SO4 1 / H2O 2

STOIC 4 MIXED CL2 -1 / NAOH -2 / NACL $2 / \mathrm{H} 2 \mathrm{O} 1 /$ \&

$\mathrm{O} 20.5$

STOIC 5 MIXED SO2 -1 / NAOH -2 / O2 - 0.5 / NA2SO4 1 \& / $\mathrm{H} 2 \mathrm{O} 1$

STOIC 6 MIXED HNO3 - 1 / NAOH - 1 / NANO3 $1 /$ H2O 1

CONV 1 MIXED 030.999

CONV 2 MIXED HCL 0.99

CONV 3 MIXED H2SO4 0.99

CONV 4 MIXED CL2 0.99

CONV 5 MIXED SO2 0.99

CONV 6 MIXED HNO3 0.99

;assumed temperature and pressure

BLOCK OZ-GEN RSTOIC

DESCRIPTION "generates ozone from air"

PARAM TEMP=100 PRES $=1$

STOIC 1 MIXED O2 -3/O3 2

CONV 1 MIXED O2 0.999

;Destruction efficiency of $96 \%$ assumed (p. 2-78 of "Description of Recommended ;Non-Thermal Mixed Waste Treatment Technologies Version 1.0," Emile A. Bernard ;temperature and pressure assumed by BWBrown 
BLOCK PHOTO-UV RSTOIC

DESCRIPTION "Photolytic oxidation of waste, see comments"

PARAM TEMP=100 PRES=1 SERIES=YES

STOIC 1 CISOLID C-S -1 / MIXED OH-RAD - 4 / CO2 $1 /$ \& $\mathrm{H} 2 \mathrm{O} 2$

STOIC 2 MIXED H2 -1/ OH-RAD - $2 / \mathrm{H} 2 \mathrm{O} 2$

STOIC 3 MIXED H2 -1 / CL2 -1 / HCL 2

CONV 1 CLSOLID C-S 0.96

CONV 2 MIXED H2 0.96

CONV 3 MIXED CL2 0.96

BLOCK VACTRAX1 RGIBBS

PARAM TEMP $=550$ PRES $=1<$ PSI $>$

PROD H2O / H2 / O2 / N2 / S / CL2 / NO / SIO2-S \& $\mathrm{S} / \mathrm{AL}$ 2O3-S S / AS / FE / HG / AG / HCL / \& $\mathrm{H} 2 \mathrm{SO} 4 / \mathrm{CH} 4 / \mathrm{NH} 3 / \mathrm{C}-\mathrm{S}$ S / BA M / CD M/CR \& $\mathrm{M} / \mathrm{PB} \mathrm{M} / \mathrm{SE} \mathrm{M}$

PROD-FRAC O2 1

; $10 \%$ excess $\mathrm{OH}$ radicals

DESIGN-SPEC AIRI

DEFINE OHROUT MOLE-FLOW STREAM=PRODUCTS SUBSTREAM=MIXED \& COMPONENT $=O H-R A D$

DEFINE OHRIN MOLE-FLOW STREAM=OH-RAD SUBSTREAM=MIXED \& COMPONENT $=O H-R A D$

SPEC "OHROUT" $(1+100 / 10)$ " TO "OHRIN

TOL-SPEC "0.101"

VARY STREAM-VAR STREAM=AIRI SUBSTREAM=MIXED \& VARIABLE=MASS-FLOW

IIMITS "0.01" "5"

DESIGN-SPEC AIR4

DEFINE OXOUT MASS-FLOW STREAM=GPCR-OUT SUBSTREAM=MIXED \& COMPONENT $=02$

DEFINE OX1 MASS-FLOW STREAM=AIR4 SUBSTREAM=MIXED \& COMPONENT $=02$

DEFINE OX2 MASS-FLOW STREAM=FILTERED SUBSTREAM=MIXED \& COMPONENT $=\mathrm{O} 2$

SPEC "OX1+OX2" TO "OXOUT* $(1+100 / 100)$ "

TOL-SPEC "0.01"

VARY STREAM-VAR STREAM=AIR4 SUBSTREAM $=$ MIXED \& VARIABLE=MASS-FLOW

LIMTTS "0.00001" "100"

;Perry's Handbook p. 20-33 has moisture contents about 0.5\% from dryers ;review meeting of $2 / 8 / 96$ : moisture content to $5 \%$

DESIGN-SPEC DRYER

DEFINE H2OMAS MASS-FLOW STREAM $=$ PLYSTBIN SUBSTREAM=MIXED \& COMPONENT $=\mathrm{H} 2 \mathrm{O}$

DEFINE MIXMAS STREAM-VAR STREAM=PLYSTBIN SUBSTREAM=MIXED \& VARIABLE=MASS-FLOW

DEFINE SOLMAS STREAM-VAR STREAM $=$ PLYSTBIN SUBSTREAM=CISOLID \& VARIABLE=MASS-FLOW

SPEC "H2OMAS" TO "(MIXMAS+SOLMAS)*0.05"

TOL-SPEC "0.001" 
VARY BLOCK-VAR BLOCK=WATERSEP SENTENCE=FRAC VARIABLE=FRACS \& D1=MIXED ID2=PLYSTBIN ELEMENT $=12$

LIMTS " 0 " "1"

;power input is calculated to be $56.49 \mathrm{~W} / \mathrm{SCFM}$ according to information

; supplied by Ben Tehranian, MK Environmental Services

$; \mathrm{lbmol} / \mathrm{hr}$ converted to SCFM by multiplying by $359 / 60$

$\ddot{*}$

$; 1 \mathrm{~W}=3.413 \mathrm{Btu} / \mathrm{hr}$

DESIGN-SPEC GPCRPOWR

DEFINE MOLOUT STREAM-VAR STREAM=GPCR-OUT SUBSTREAM=MIXED \& VARIABLE=MOLE-FLOW

DEFINE POWER BLOCK-VAR BLOCK=GPCR VARIABLE=DUTY \& SENTENCE $=$ PARAM

SPEC "POWER" TO "MOLOUT*359/60*56.49*3.413"

TOL-SPEC "0.01"

VARY BLOCK-VAR BLOCK $=$ GPCR VARLABLE $=D U T Y ~ S E N T E N C E=P A R A M$

LIMITS "0" "10000000"

;conversation with Daryoush Bahar 3/6/96 - dry basis used for grout calculation ;because with System 3, too much water exists, which results in too much grout ;being calculated

DESIGN-SPEC GROUT

DEFINE R1 STREAM-VAR STREAM=GROUTRN SUBSTREAM=MIXED \& VARIABLE=MASS-FLOW

DEFINE R2 STREAM-VAR STREAM=GROUTRIN SUBSTREAM=CISOLID \& VARIABLE=MASS-FLOW

DEFINE R3 MASS-FLOW STREAM=GROUTRN SUBSTREAM=MIXED \& COMPONENT $=\mathrm{H} 2 \mathrm{O}$

DEFINE GROUT STREAM-VAR STREAM=GROUTDRY SUBSTREAM=CISOLID \& VARIABLE $=$ MASS-FLOW

F $\quad$ EXCES $=\mathrm{R} 3-0.6^{*}(\mathrm{R} 1+\mathrm{R} 2-\mathrm{R} 3)$

F IF(EXCES.LT.0.) EXCES $=0$.

SPEC "GROUT "TO " $1.4^{*}(\mathrm{R} 1+\mathrm{R} 2-\mathrm{R} 3)+2.333^{*} \mathrm{EXCES}^{\mathrm{N}}$

TOL-SPEC "0.1"

VARY STREAM-VAR STREAM=GROUTDRY SUBSTREAM=CISOLD \& VARIABLE=MASS-FLOW

LIMTS "0.1" "6000"

DESIGN-SPEC GROUTH2O

DEFINE R1 STREAM-VAR STREAM=GROUTRIN SUBSTREAM=MIXED \& VARIABLE=MASS-FLOW

DEFINE R2 STREAM-VAR STREAM=GROUTRIN SUBSTREAM=CISOLD \& VARIABLE=MASS-FLOW

DEFINE R3 MASS-FLOW STREAM=GROUTRIN SUBSTREAM=MIXED \& COMPONENT $=$ H2O

DEFINE H2OFLO STREAM-VAR STREAM=GROUTH2O SUBSTREAM=MIXED \& VARIABLE $=$ MASS-FLOW

$F \quad$ EXCES $=R 3-0.6 *(R 1+R 2-R 3)$

F IF(EXCES.GT.0.) EXCES $=0.0$

C SETS EXCESS WATER TO O IF WATER IS ACTUALLY EXCESS

C WATER REQUIRED, IF ANY, IS RETURNED AS A NEGATTVE NUMBER

SPEC "H2OFLO" TO "-EXCES"

TOL-SPEC " 0.01 " 


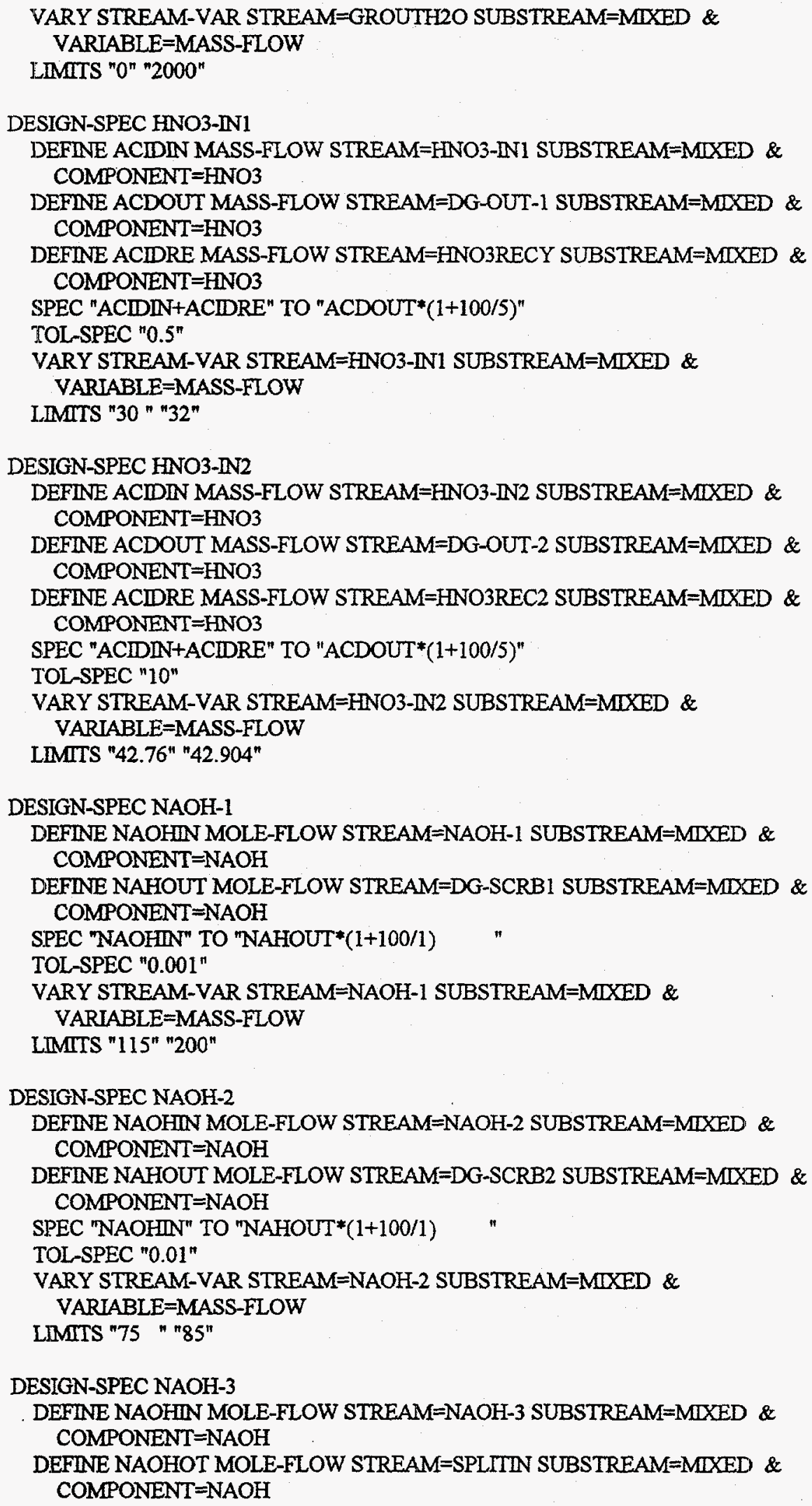




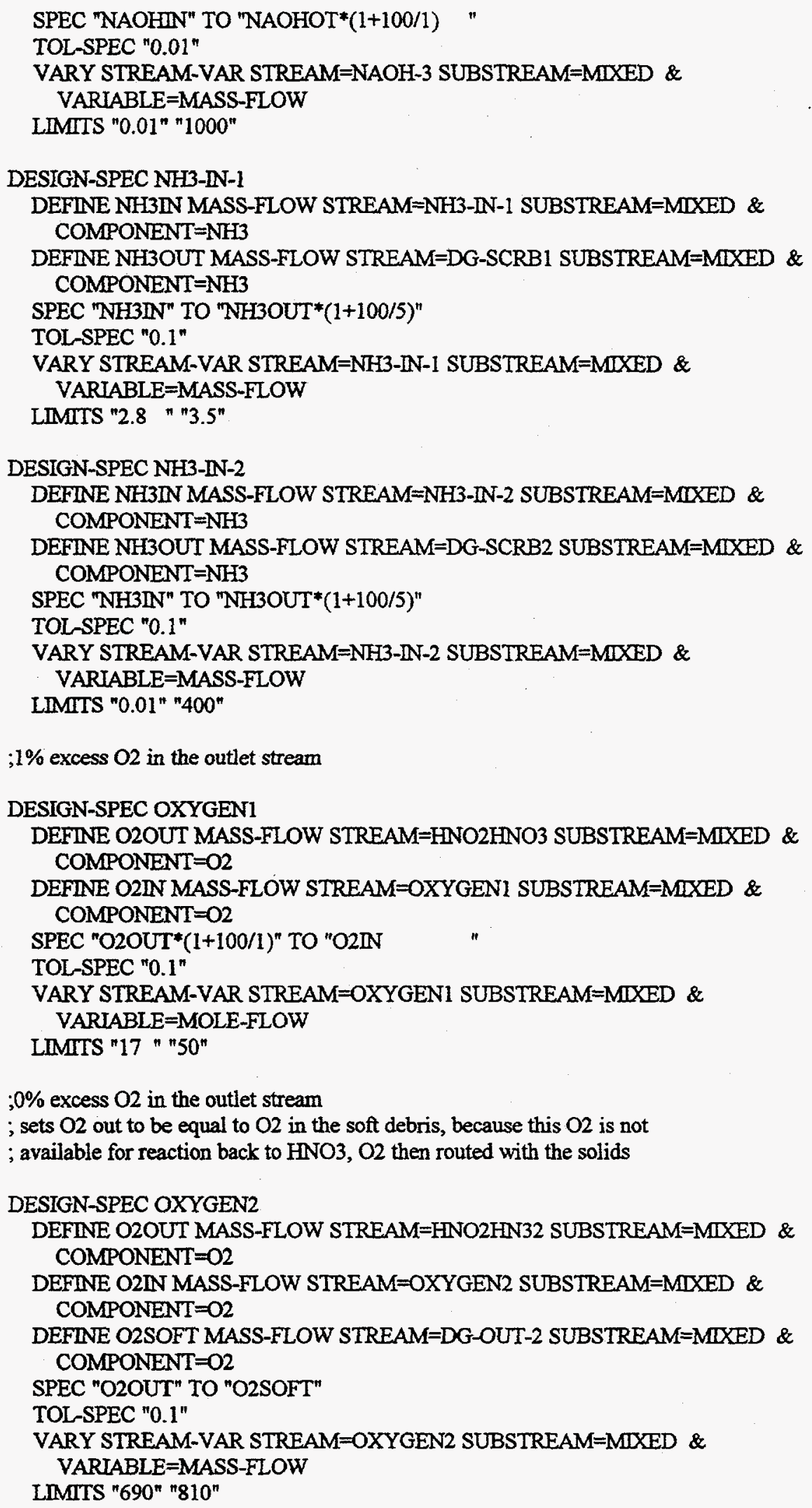









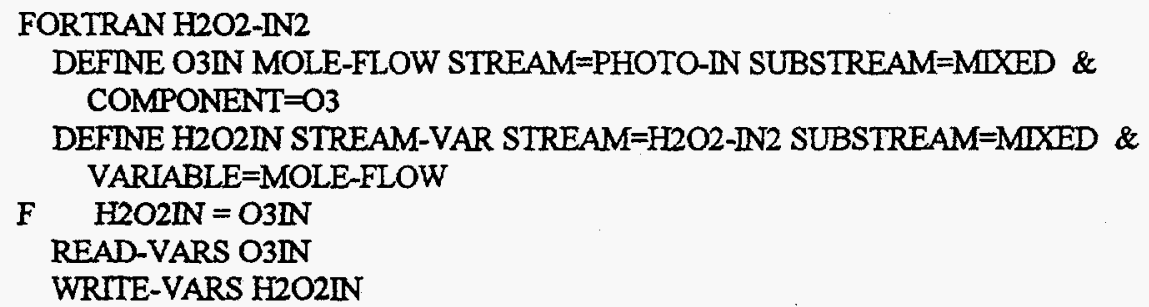

; constant is obtained by taking $1200 \mathrm{ft} 3 / 1200 \mathrm{lb}$ and mult. by $0.0727 \mathrm{lb} / \mathrm{ft} 3$

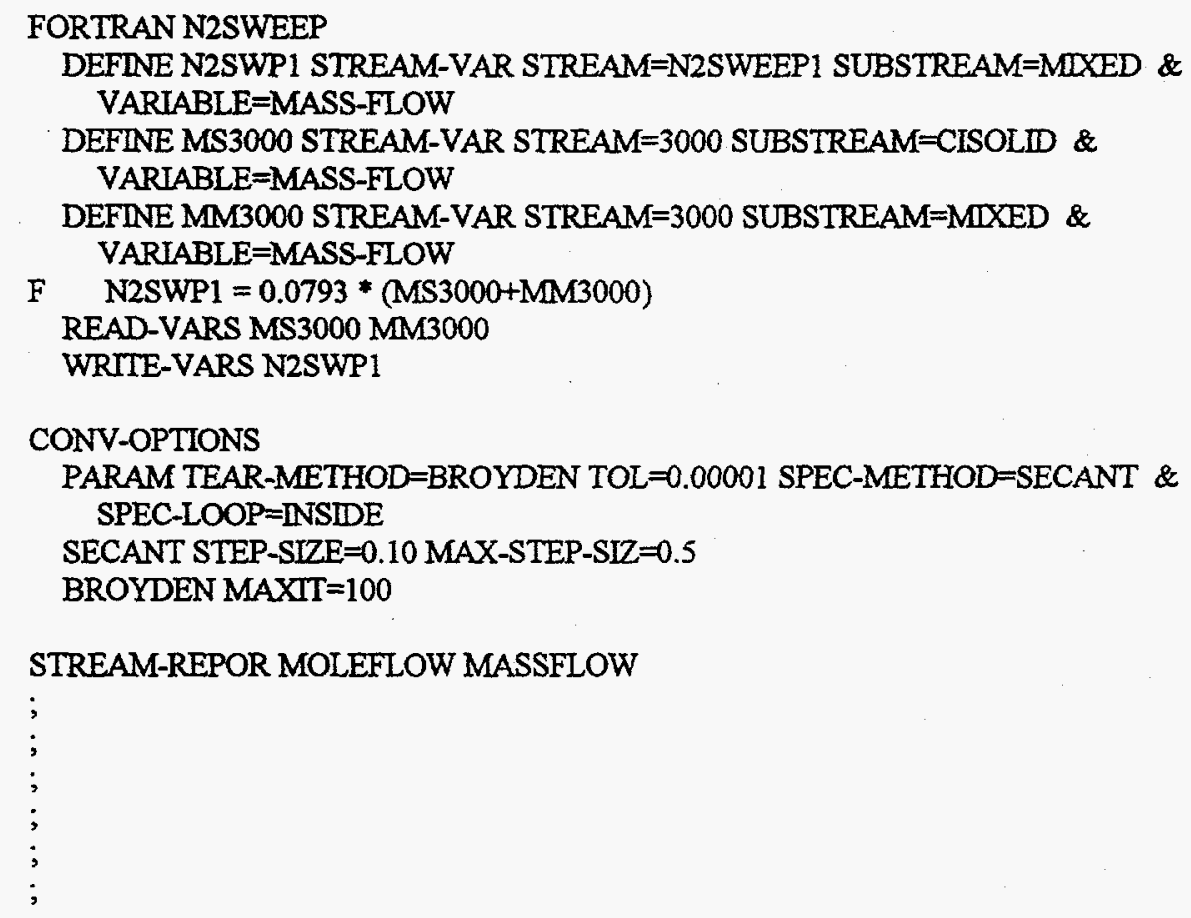




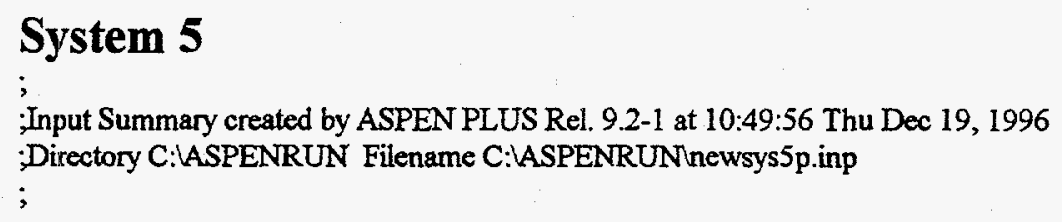


HCL HCL HCL /

FECL3 FECL3 FECL3/

$\mathrm{H} 2 \mathrm{SO}_{4} \mathrm{H}_{2} \mathrm{SO}_{4} \mathrm{H} 2 \mathrm{SO} 4 /$

$\mathrm{NA} 2 \mathrm{SO} 4 \mathrm{NA} 2 \mathrm{SO} 4 \mathrm{NA} 2 \mathrm{SO} 4 /$

$\mathrm{CH} 4 \mathrm{CH}_{4} \mathrm{CH} 4 /$

POLYMER C2H4 POLYMER/

CEMENT CAAL2SIO6 CEMENT /

$\mathrm{CASO} 4 \mathrm{CASO} 4 \mathrm{CASO}_{4} /$

CUCU CUI

"FE(OH)3" "FE(OH)3" "FE(OH)3" /

OH-RAD OH OH-RAD

FLOWSHEET APC-SYS

BLOCK GPCR IN=FILTERED AIR 4 OUT =GPCR-OUT

BLOCK FILTER IN=AQ-OFGAS EQUIL1 VACGAS-1 SOILVAPR EQUIL2 \& OUT=FILTERED METALS

BLOCK NEUTRAL3 $\mathbb{N}=$ =NAOH-3 GPCR-OUT OUT=SPLITIN

BLOCK BLOWDOWN $\mathbb{N}=$ =SPLITIN OUT $=$ FLASH-IN SALTSOLN

BLOCK FLASH2 IN=FLASH-IN OUT =HEATERIN MOISTURE

BLOCK HETRHEPA IN=HEATERN OUT=OFFGAS

FLOWSHEET AQUEOUS

BLOCK AQ-SPLIT IN=AQ-OUT OUT=AQ-SLUDG AQ-OFGAS AQ-RECYC

BLOCK OZ-GEN IN=AIR1 OUT=OZONE

BLOCK CONCENT8 IN=SALTSOLN OUT=AQ-RECY2 CONCENT8

BLOCK PHOTOMIX IN=VACLIQ-1 METLRECY 1000 SPENTH2O H2ORECYC \& OZONE MOISTURE H2O2-IN2 SPLT-H2O SPLTH2O2 HPWATOUT \& EXCESH2O XX-NAOH AQ-RECY2 OUT=X-PHOTIN

BLOCK OH-GEN IN=PHOTO-IN OUT $=O H-R A D$

BLOCK PHOTO-UV IN=OH-RAD OUT=PRODUCTS

BLOCK OZ-DECOM IN=PRODUCTS OUT=AQ-OUT

BLOCK OILSEP IN=X-PHOTIN OUT=PHOTO-IN X-ORGS

FLOWSHEET BULKSOII

BLOCK SOILWASH IN=4000 WASH-IN OUT=SPENTH2O SOIL VAPR \& SOIL-ORG TRETSOIL

BLOCK H2OSEP IN=WARMSOIL OUT=EXCESH2O DRYSOIL

BLOCK SOLDRYER $\mathbb{N}=$ TRETSOIL OUT=WARMSOIL

FLOWSHEET GROUT

BLOCK GROUTER IN=GROUTDRY GROUTRIN GROUTH2O OUT=GROUTOUT

BLOCK GROUTMIX IN=5000COMP WASHDEBR PBGLOVES CWOSLD2 \& DRYSOIL OUT $=$ GROUTRIN

FLOWSHEET HG-AMAL

BLOCK HG-AMALG IN=7000-HG ADDITIVE VAC-HG-1 OUT=HG-OUT

FLOWSHEET METLDCON

BLOCK MET-DCON IN=METLWASH 5000METL OUT=METALOUT METLRECY

FLOWSHEET ORGDEST

BLOCK EQUIL-1 IN=CWO-GAS OUT $=$ EQUIL 1

BLOCK CWO-1 IN=ACD-IN 20003000 OXYGEN1 VACORG-1 SOIL-ORG \& X-ORGS OUT $=$ CWO-OUT

BLOCK CWOSPLT IN=CWO-OUT SCRBSALT OUT=CWO-LIQ CWO-SLD \& CWO-GAS

BLOCK NEUTRAL1 IN=NAOH-1 CWO-LIQ OUT=SCRBOUT 1

BLOCK SALTFLTR IN=SCRBOUT1 OUT $=$ SPLT-H2O SCRBSALT 
FLOWSHEET PB-RECVR

BLOCK PB-DECON IN=PBBRICKS PB-WATER OUT=LEAD-OUT PBSLUDGE

BLOCK PB-SPLIT IN=7000-PB OUT=PBBRICKS PBGLOVES

FLOWSHEET POLYMER

BLOCK POLYSTAB IN=PLYSTBNN POLYETHL OUT=POLY-OUT

BLOCK POLY-MIX IN=3000POLY CWO-SLD AQ-SLUDG CONCENT8 \& METALS VACSOL-1 OUT $=$ MIXED-N

BLOCK DRYER IN=MIXED-IN OUT=HOT-MIX

BLOCK WATERSEP IN=HOT-MIX OUT=H2ORECYC PLYSTBDN

FLOWSHEET RES-SLUD

BLOCK VACTRAX1 IN=3000 PBSLUDGE N2SWEEP1 OUT=VACOUT 1

BLOCK EQUIL-2 IN=CWOGAS2 OUT $=$ EQUIL2.2

BLOCK NEUTRAL2 IN=NAOH-2 CWOLIQ2 OUT=SCRBOUT2

BLOCK CWO-2 IN=ACID-IN2 OXYGEN2 5000SOFT OUT $=$ CWO-OUT2

BLOCK CWOSPL $2 \mathrm{~N}=$ CWO-OUT2 SCRBSLT2 OUT $=$ CWOLIQ2 CWOSLD2 \& CWOGAS2

BLOCK HP-WASH IN $=$ H2OWASH 50000 OPEN OUT $=$ WASHDEBR HPWATOUT

BLOCK VACSEP-1 $\mathrm{N}$ =VACOUT-1 XYLEACH 1 OUT=VACGAS-1 VACORG-1 \& VACSOL-1 VAC-HG-1 VACLIQ-1

BLOCK SALTFLT2 IN=SCRBOUT2 OUT=SPLTH2O2 SCRBSLT2

FLOWSHEET SPECIAL

BLOCK SPESHULL $\mathbb{N}=6000$ OUT $=$ SPEC-OUT

PROPERTIES SOLIDS

PROPERTIES IDEAL

USER-PROPS DRUSR2 123

PROP-DATA U-1

N-UNITS ENG PRESSURE=ATM

PROP-LIST TC

PVAL "FE(OH)3" 100

PROP-DATA U-2

IN-UNITS ENG PRESSURE $=$ ATM

PROP-LIST PLXANT

PVAL "FE(OH)3" 7.511 -14062210000001000

PROP-DATA U-2

IN-UNITS ENG PRESSURE $=A T M$

PROP-LIST DHVLWT

PVAL "FE(OH)3" 110.3800

;Tables dated 04/24/96 from Daryoush Bahar, MK, (415) 442-7688

; Glass inerts assumed to be $50 \% \mathrm{SiO} 2$ and $50 \% \mathrm{Al} 2 \mathrm{O} 3$ for all waste streams.

STREAM 1000

SUBSTREAM MIXED TEMP $=68$ PRES $=1$ MASS-FLOW $=60.8$

MASS-FLOW H2 $3613<\mathrm{KG} / \mathrm{HR}>/ \mathrm{O} 2172053<\mathrm{KG} / \mathrm{HR}>/ \mathrm{N} 2$ \& $50211<\mathrm{KG} / \mathrm{HR}>/ \mathrm{H} 2 \mathrm{O} 2456410<\mathrm{KG} / \mathrm{HR}>/ \mathrm{AS} 5647<\mathrm{KG} / \mathrm{HR}>$ \& / BA $2823<\mathrm{KG} / \mathrm{HR}>$ / CD $42352<\mathrm{KG} / \mathrm{HR}>$ / CR \& $56469<\mathrm{KG} / \mathrm{HR}>/ \mathrm{PB} 14117<\mathrm{KG} / \mathrm{HR}>/ \mathrm{HG} 14117<\mathrm{KG} / \mathrm{HR}>$ / \& SE $2823<\mathrm{KG} / \mathrm{HR}>/$ AG $2823<\mathrm{KG} / \mathrm{HR}>$

; Tables dated 04/24/96 from MK 


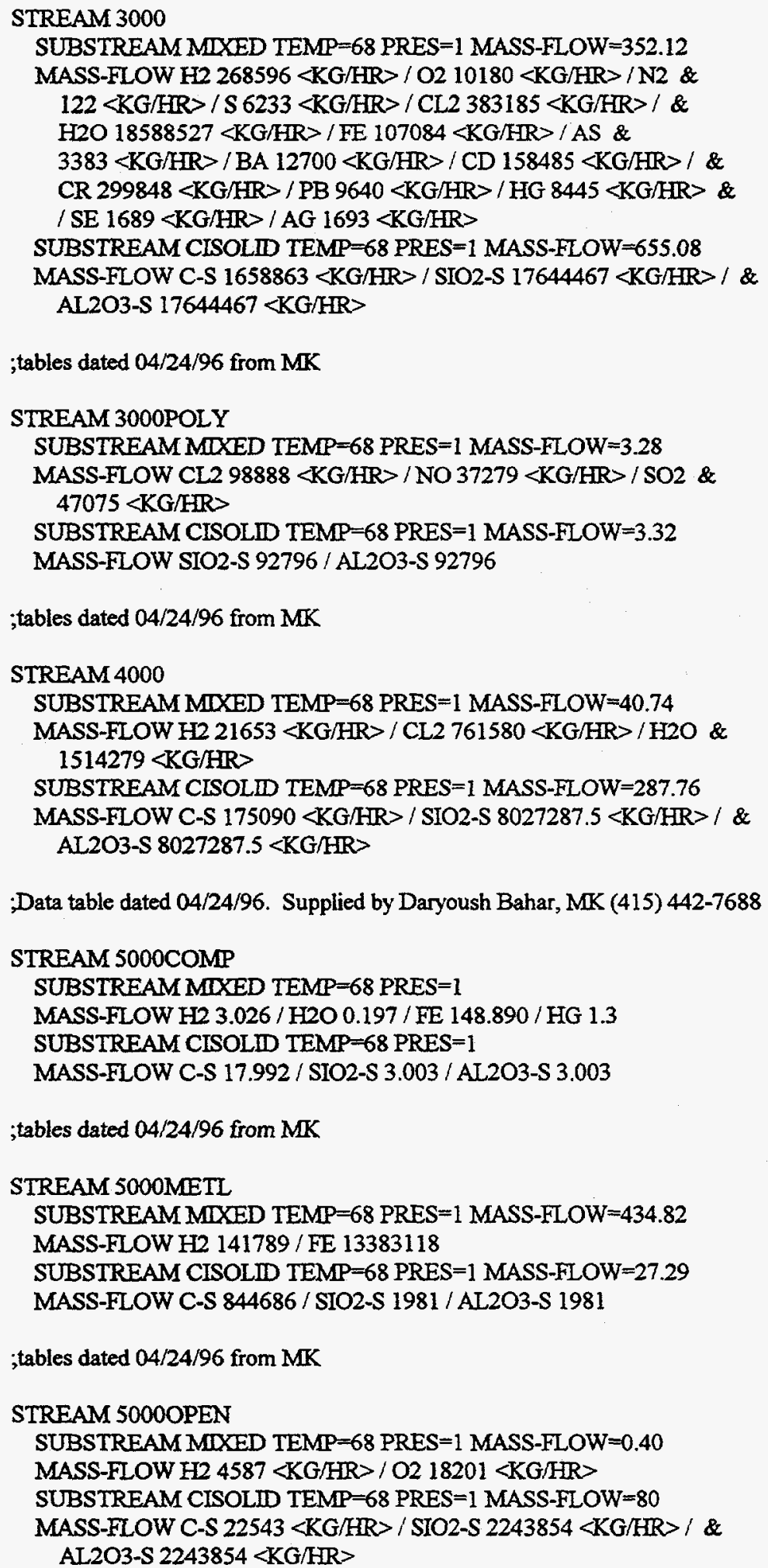

;tables dated 04/30/96, used mass flow $\mathrm{lb} / \mathrm{hr}$ rather than $\mathrm{kg}$ in waste inventory 


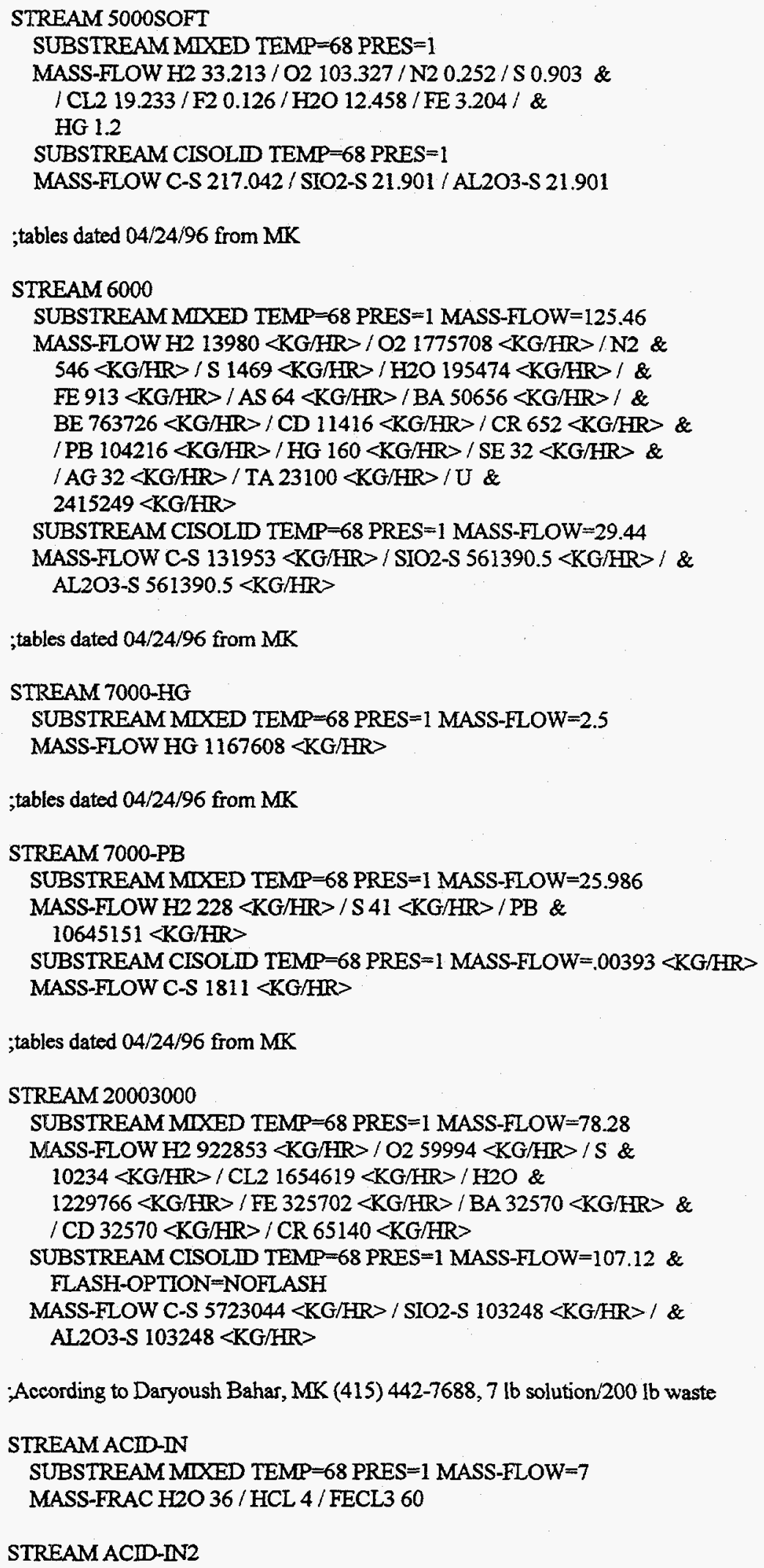

STREAM ACD-IN2 
SUBSTREAM MIXED TEMP=68 PRES=1 MASS-FLOW=15.21

MASS-FRAC H2O 36 / HCL 4 / FECL3 60

STREAM ADDITIVE

SUBSTREAM MIXED TEMP $=68$ PRES $=1$

MOLE-FLOW CU 0.0291885

STREAM AIR1

SUBSTREAM MIXED TEMP $=68$ PRES $=1$ MASS-FLOW $=90.19$

MOLE-FRAC O2 $0.21 / \mathrm{N} 20.79$

STREAM AR4

SUBSTREAM MIXED TEMP $=68$ PRES $=1$ MASS-FLOW $=0.0001$

MOLE-FRAC O2 $0.21 / \mathrm{N} 20.79$

STREAM GROUTDRY

SUBSTREAM MIXED TEMP $=68$ PRES $=1$

SUBSTREAM CISOLID TEMP $=68$ PRES $=1$ MASS-FLOW $=1$ MASS-FRAC CEMENT 1

STREAM GROUTH2O

SUBSTREAM MIXED TEMP $=68$ PRES $=1$ MASS-FLOW $=0.000001$

MASS-FRAC $\mathrm{H} 2 \mathrm{O} 1$

STREAM H2O2-IN2

SUBSTREAM MIXED TEMP=68 PRES=1 MOLE-FLOW=1

MASS-FRAC $\mathrm{H}_{2} \mathrm{O} 21$

; flow rate set to $5 \%$ of open debris, all $\mathrm{H} 2 \mathrm{O}$ goes to grout

STREAM H2OWASH

SUBSTREAM MIXED TEMP=68 PRES=1 MASS-FLOW $=12$

MASS-FRAC H2O 1

STREAM HG-OUT

SUBSTREAM MIXED TEMP $=68$ PRES $=1$

MOLE-FLOW CU 0.0291885

;Water set to $112 / 468$ times the bulk metal flow rate, from Figure 3-22

; $\quad$ (page 3-46) in ITTS Phase 2 Final Report

STREAM METLWASH

SUBSTREAM MIXED TEMP $=68$ PRES $=1$ MASS-FLOW=94.327

MASS-FRAC $\mathrm{H} 2 \mathrm{O} 1$

;3/26/96 Daryoush Bahar uses 1200 SCFH N2 for $1100 \mathrm{lb} / \mathrm{h}$ debris

STREAM N2SWEEP1

SUBSTREAM MIXED TEMP $=68$ PRES $=1$ VOLUME-FLOW $=1200$

MOLE-FRAC N2 1

;According to Delphi flowsheet D-1002A, $50 \% \mathrm{NaOH}$ solution

STREAMNAOH-1

SUBSTREAM MIXED TEMP=68 PRES=1 MASS-FLOW=109

MASS-FRAC H2O 0.5 / NAOH 0.5

;According to Delphi flowsheet D-1002A, $50 \% \mathrm{NaOH}$ solution 


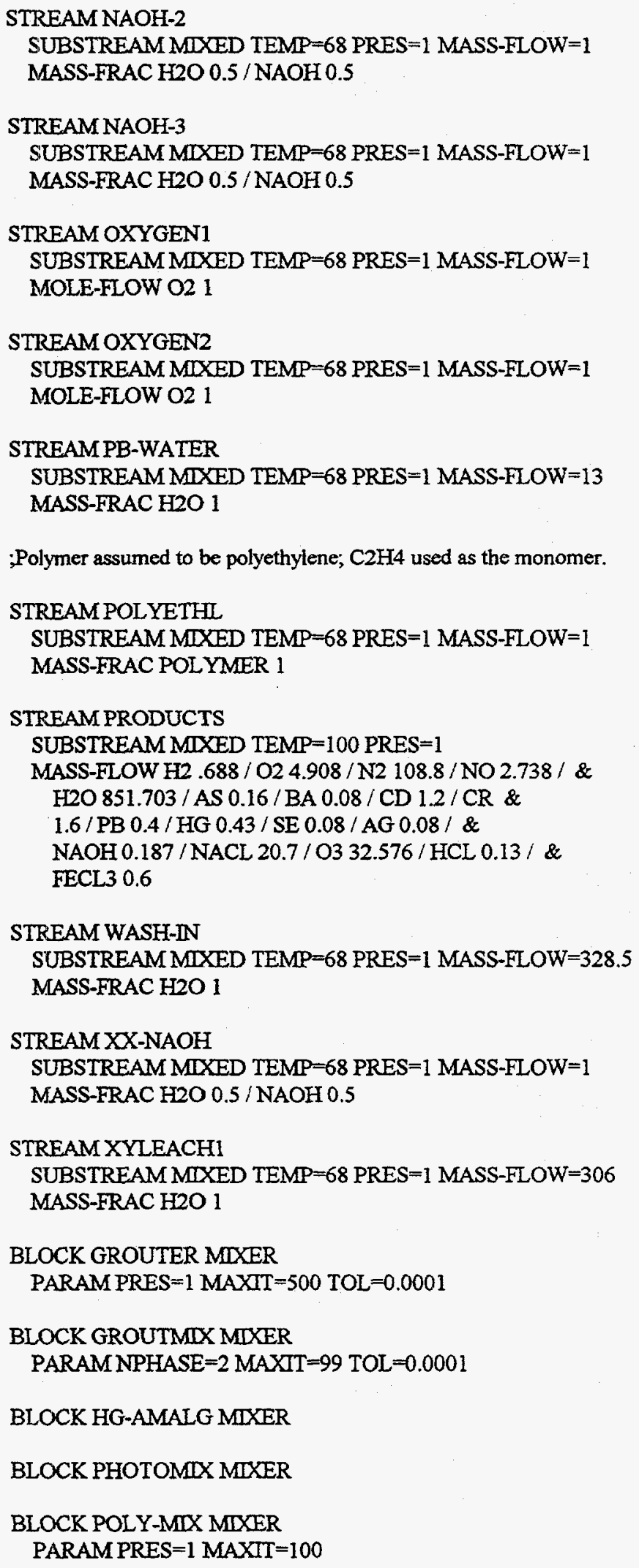


BLOCK POLYSTAB MIXER

DESCRIPTION "for the moment, this is only a mixer"

BLOCK-OPTION ENERGY-BAL=YES

\section{BLOCK SPESHULL MIXER}

BLOCK AQ-SPLTT SEP

DESCRIPTION "this separates water, gases, and solids"

FRAC STREAM $=$ AQ-SLUDG SUBSTREAM $=$ MIXED COMPS $=\mathrm{C} C-\mathrm{S}$ H2 $\mathrm{O} 2$ \& N2 S CL2 F2 NO SO2 H2O SIO2 SIO2-S AL2O3 AL2O3-S \&

FE AS BA CD CR PB HG SE AG NAOH NACL $\mathrm{CO} 2 \mathrm{CO} O 3$ \& HCL FECL3 H2SO4 NA2SO4 CH4 POLYMER CEMENT CASO4 \& "FE(OH)3" FRACS $=110001110000.990 .99 \&$ $0.990 .9911111111111000010 \&$ 101111

FRAC STREAM=AQ-SLUDG SUBSTREAM=CISOLD COMPS=C-S SIO2-S \& AL2O3-S CEMENT FRACS $=0.990 .990 .991$

FRAC STREAM=AQ-OFGAS SUBSTREAM $=$ MIXED COMPS $=\mathrm{C} C-\mathrm{S}$ H2 $\mathrm{O} 2$ \& N2 S CL2 2 2 NO SO2 H2O SIO2 SIO2-S AL2O3 AL2O3-S \& FE AS BA CD CR PB HG SE AG NAOH NACL $\mathrm{CO} 2 \mathrm{CO} O 3 \&$ HCL FECL3 H2SO4 NA2SO4 CH4 POLYMER CEMENT CASO4 \& FRACS $=001110001000000000 \&$ 0000000011100001000

FRAC STREAM=AQ-OFGAS SUBSTREAM=CISOLID COMPS=C-S SIO2-S \& AL2O3-S CEMENT FRACS $=0000$

BLOCK BLOWDOWN SEP

FRAC STREAM=FLASH-IN SUBSTREAM=MIXED COMPS=C C-S H2 O2 \& N2 S CL2 F2 NO SO2 H2O SIO2 SIO2-S AL2O3 AL2O3-S \&

FE AS BA CD CR PB HG SE AG NAOH NACL CO2 $\mathrm{CO} O 3$ \& HCL FECL3 $\mathrm{H} 2 \mathrm{SO} 4 \mathrm{NA} 2 \mathrm{SO} 4 \mathrm{CH} 4 \mathrm{POLYMER} \mathrm{CEMENT} \mathrm{CASO4} \mathrm{\&}$ FRACS $=11111111110.91111111 \&$

1111110011111101001

FRAC STREAM $=$ FLASH-IN SUBSTREAM $=$ CISOLID COMPS $=$ C-S SIO2-S \& AL2O3-S CEMENT FRACS $=0000$

; Hg retention in solid set to 0.95 to allow $5 \%$ removal to aqueous phase

\section{BLOCK CWOSPL2 SEP}

DESCRIPTION "for the moment, assumes complete separation"

FRAC STREAM=CWOLIQ2 SUBSTREAM=MIXED COMPS=C C-S H2 O2 \& N2 S CL2 F2 NO H2O SIO2 SIO2-S AL2O3 AL2O3-S FE AS \& BA CD CR PB HG SE AG NAOH NACL $\mathrm{CO} 2 \mathrm{CO} 03 \mathrm{HCL} \&$ FECL3 $\mathrm{H} 2 \mathrm{SO} 4 \mathrm{NA} 2 \mathrm{SO} 4 \mathrm{CH} 4$ POL YMER CEMENT CASO4 CU FRACS $=$ \& $00000000010000000000 \&$ 0.050011000111100010

FRAC STREAM $=$ CWOLIQ2 SUBSTREAM $=$ CISOLDD $C O M P S=C-S$ STO2-S \& AL2O3-S CEMENT FRACS $=0000$

FRAC STREAM $=$ CWOSLD2 SUBSTREAM $=$ MIXED COMPS $=\mathrm{C}$ C-S H2 O2 \& N2 S CL2 F2 NO H2O SIO2 SIO2-S AL2O3 AL2O3-S FE AS \& BA CD CR PB HG SE AG NAOH NACL CO2 CO O3 HCL \& FECL3 $\mathrm{H} 2 \mathrm{SO} 4 \mathrm{NA} 2 \mathrm{SO} 4 \mathrm{CH} 4$ POL YMER CEMENT CASO4 CU \& "FE(OH)3" FRACS=110101000011111\& $111110.9511000000000000 \&$ 001

FRAC STREAM=CWOSLD2 SUBSTREAM=CISOLID COMPS=C-S SIO2-S \& AL2O3-S CEMENT FRACS $=1111$ 


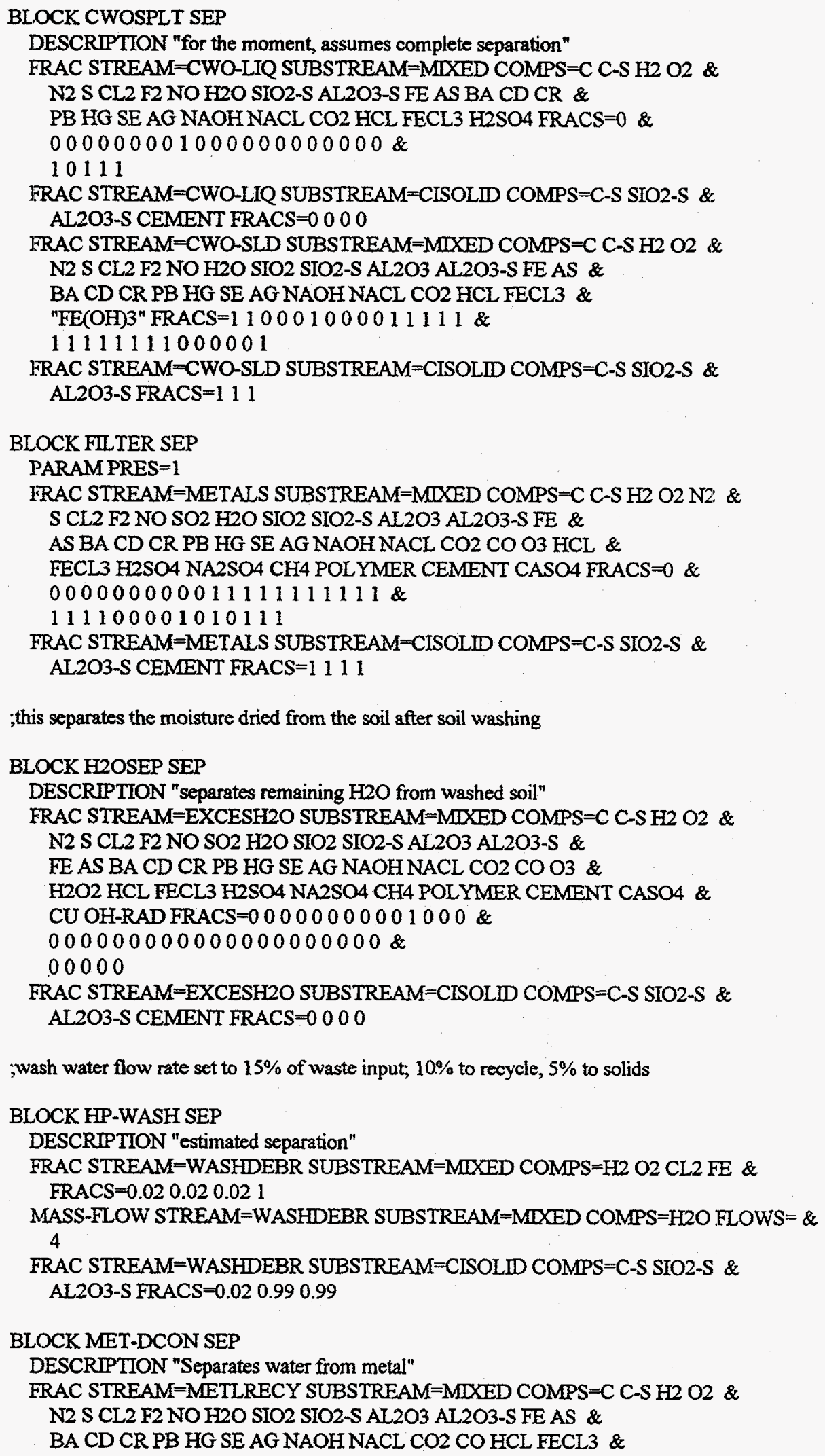


$\mathrm{H} 2 \mathrm{SO} 4 \mathrm{NA} 2 \mathrm{SO} 4 \mathrm{CH} 4$ POLYMER CEMENT FRACS $=001000$ \& $000100000000000000000 \&$ 0000000

FRAC STREAM=METLRECY SUBSTREAM=CISOLID COMPS=C-S SIO2-S \& AL2O3-S CEMENT FRACS $=11110$

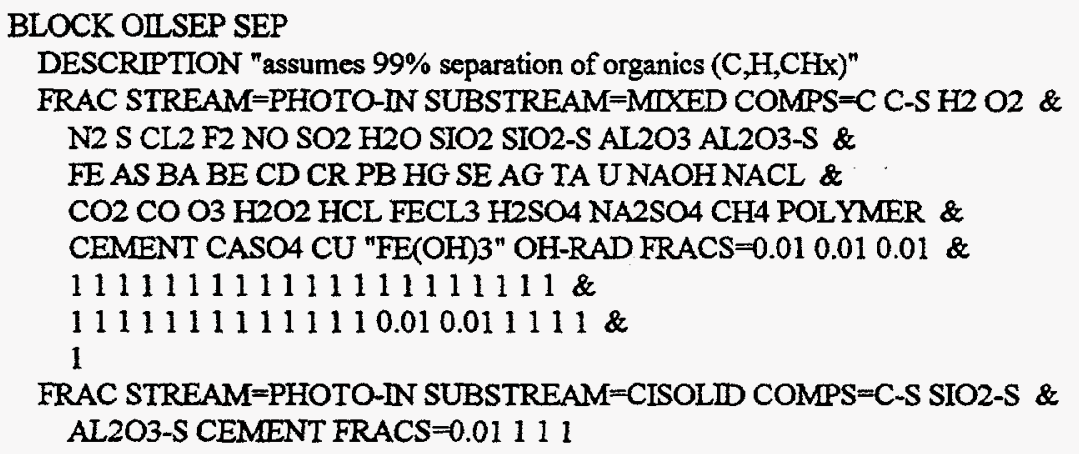

BLOCK PB-DECON SEP

FRAC STREAM $=$ LEAD-OUT SUBSTREAM $=$ MIXED COMPS $=$ PB FRACS $=1$ FRAC STREAM=LEAD-OUT SUBSTREAM=CISOLD COMPS=C-S SIO2-S \& AL2O3-S CEMENT FRACS $=0000$

BLOCK PB-SPLIT SEP

FRAC STREAM $=$ PBBRICKS SUBSTREAM $=$ MIXED COMPS=H2 $S$ FRACS $=\&$ 0.810 .81

MASS-FLOW STREAM $=$ PBBRICKS SUBSTREAM $=$ MIXED COMPS $=$ PB FLOWS $=\&$ 21

FRAC STREAM=PBBRICKS SUBSTREAM=CISOLID COMPS=C-S SIO2-S \& AL2O3-S FRACS $=0.810 .810 .81$

BLOCK SALTFLT2 SEP

FRAC STREAM=SPLTH2O2 SUBSTREAM=MIXED COMPS=C C-S H2 O2 \& N2 S CL2 F2 NO SO2 H2O SIO2 SIO2-S AL2O3 AL2O3-S \& FE AS BA CD CR PB HG SE AG NAOH NACL CO2 $\mathrm{CO} O 3$ \& $\mathrm{H} 2 \mathrm{O} 2 \mathrm{HCL}$ FECL3 $\mathrm{H} 2 \mathrm{SO} 4 \mathrm{NA} 2 \mathrm{SO} 4 \mathrm{CH} 4$ POLYMER CEMENT CASO4 \& $\mathrm{CU}$ "FE(OH)3" OH-RAD FRACS=11111111111\&

$111111111111111111111 \&$ 111111101

FRAC STREAM=SPLTH2O2 SUBSTREAM=CISOLDD COMPS=C-S SIO2-S \& AL2O3-S CEMENT FRACS $=1111$

BLOCK SALTFLTR SEP

FRAC STREAM=SPLT-H2O SUBSTREAM=MIXED COMPS $=\mathrm{C}$ C-S H2 O2 \& N2 S CL2 F2 NO SO2 H2O SIO2 $\mathrm{SIO}_{2} \mathrm{~S}$ AL2O3 AL2O3-S \& FE AS BA CD CR PB HG SE AG NAOH NACL $\mathrm{CO} 2 \mathrm{CO} O 3$ \& H2O2 HCL FECL 3 H2SO4 NA2SO4 CH4 POL YMER CEMENT CASO4 \& CU "FE(OH)3" OH-RAD FRACS=111111111111 \& $111111111111111111111 \&$ 111111101

FRAC STREAM=SPLT-H2O SUBSTREAM=CISOLID COMPS=C-S SIO2-S \& AI2O3-S CEMENT FRACS=1111

BLOCK SOLW WASH SEP

FRAC STREAM=SPENTH2O SUBSTREAM=MIXED COMPS=C C-S H2 O2 \& N2 S CL2 F2 NO SO2 $\mathrm{H} 2 \mathrm{O}$ SIO2 $\mathrm{SIO} 2-\mathrm{S} \mathrm{AL} 2 \mathrm{O} 3 \mathrm{AL} 2 \mathrm{O} 3-\mathrm{S}$ \& FE AS BA BE CD CR PB HG SE AG TA U NAOH NACL \& 
$\mathrm{CO} 2 \mathrm{CO} O 3 \mathrm{H} 2 \mathrm{O} 2 \mathrm{HCL}$ FECL3 H2SO4 NA2SO4 CH4 POLYMER \& CEMENT CASO4 CU FRACS $=000000000000 \&$ 000000000000000000000 \& 000000000

FRAC STREAM=SPENTH2O SUBSTREAM=CISOLID COMPS=C-S SIO2-S \& AL2O3-S CEMENT FRACS $=0000$

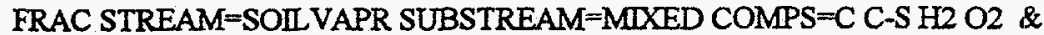
N2 S CL2 F2 NO SO2 H2O SIO2 SIO2-S AL2O3 AL2O3-S \& FE AS BA BE CD CR PB HG SE AG TA U NAOH NACL \& $\mathrm{CO} 2 \mathrm{CO} O 3 \mathrm{H} 2 \mathrm{O} 2 \mathrm{HCL}$ FECL $3 \mathrm{H} 2 \mathrm{SO} 4 \mathrm{NA} 2 \mathrm{SO} 4 \mathrm{CH} 4$ POLYMER \& CEMENT CASO4 CU "FE(OH)3" OH-RAD FRACS $=000000$ \& 000000000000000000000 \& 00000000000000000

FRAC STREAM=SOILVAPR SUBSTREAM=CISOLID COMPS=C-S SIO2-S $\&$ AL2O3-S CEMENT FRACS $=0000$

FRAC STREAM=SOIL-ORG SUBSTREAM $=\mathrm{MIXED}$ COMPS $=\mathrm{C}$ C-S H2 O2 \& N2 S CL2 F2 NO H2O SIO2 SIO2-S AL2O3 AL2O3-S FE AS \& BA CD CR PB HG SE AG NAOHNACL CO2 CO O3 HCL \& FECL3 H2SO4 NA2SO4 CH4 POLYMER CEMENT CASO4 CU FRACS $=\&$ 000.950 .95000 .9510000000000 \& 0000000000100011000

FRAC STREAM=SOIL-ORG SUBSTREAM=CISOLID COMPS=C-S SIO2-S \& AL2O3-S CEMENT FRACS $=0.95000$

;assumes $50 \%$ desorption of $\mathrm{C}, \mathrm{H} 2$

BLOCK VACSEP-1 SEP

FRAC STREAM=VACGAS-1 SUBSTREAM=MIXED COMPS=C C-S H2 O2 \& N2 S CL2 F2 NO H2O SIO2 SIO2-S AL2O3 AL2O3-S FE AS \& BA CD CR PB HG SE AG NAOH NACL CO2 CO O3 HCL \& FECL3 H2SO4 NA2SO4 CH4 POLYMER CEMENT CASO4 CU FRACS $=$ \& 000010001000000000000 \& 0000101000000000

FRAC STREAM=VACGAS- 1 SUBSTREAM=CISOLID COMPS=C-S SIO2-S \& AL2O3-S CEMENT FRACS $=0000$

FRAC STREAM=VACORG-1 SUBSTREAM=MIXED COMPS=C C-S H2 O2 \& N2 S CL2 F2 NO H2O SIO2 SIO2-S AL2O3 AI2O3-S FE AS \& BA CD CR PB HG SE AG NAOH NACL CO2 CO O3 HCL \& FECL3 H2SO4 NA2SO4 CH4 POLYMER CEMENT CASO4 CU FRACS= \& 000.50000 .50 .500000000000 \& 000000010000011000

FRAC STREAM=VACORG-1 SUBSTREAM=CISOLID COMPS=C-S SIO2-S \&z AL2O3-S CEMENT FRACS $=0.5000$

FRAC STREAM=VACSOL -1 SUBSTREAM $=$ MIXED COMPS $=\mathrm{C} \mathrm{C-S} \mathrm{H2} \mathrm{O2} \mathrm{\&}$ N2 S CL2 F2 NO SIO2 SIO2-S AL2O3 AL2O3-S FE AS BA \& CD CR PB SE AG NAOH NACL CO2 $\mathrm{CO} O 3 \mathrm{HCL}$ FECL3 \& $\mathrm{H} 2 \mathrm{SO} 4 \mathrm{NA} 2 \mathrm{SO} 4 \mathrm{CH} 4$ POLYMER CEMENT CASO4 CU FRACS $=00$ \& 0.51010001111111111111 \& 1000010100111

MASS-FLOW STREAM=VACSOL- 1 SUBSTREAM=MIXED COMPS $=$ H2O FLOWS $=$ \& 306

FRAC STREAM=VACSOL-1 SUBSTREAM=CISOLID COMPS=C-S SIO2-S \& AL2O3-S CEMENT FRACS=0.5 111

FRAC STREAM=VAC-HG-1 SUBSTREAM $=$ MIXED COMPS=C C-S H2 O2 \& N2 S CL2 F2 NO H2O SIO2 SIO2-S AL2O3 AL2O3-S FE AS \& BA CD CR PB HG SE AG NAOH NACL CO2 CO O3 HCL \& FECL 3 H2SO4 NA2SO4 CH4 POLYMER CEMENT CASO4 CU FRACS $=\&$ 0000000.50 .500000000000 \& 
010000000000000000

FRAC STREAM $=$ VAC-HG-1 SUBSTREAM $=$ CISOLID COMPS $=$ C-S SIO2-S \& AL2O3-S CEMENT FRACS $=0000$

;Perry's Handbook p. 20-33 shows moisture contents of below $1 \%$ from dryers.

BLOCK WATERSEP SEP

DESCRIPTION "This separates water in polymer stab. subsystem"

FRAC STREAM=PLYSTBIN SUBSTREAM=MIXED COMPS=C C-S H2 O2 \& N2 S CL2 F2 NO H2O SIO2 SIO2-S AL2O3 AL2O3-S FE AS \& BA CD CR PB HG SE AG NAOH NACL CO2 CO O3 HCL \& FECL3 H2SO4 NA2SO4 CH4 POLYMER CEMENT CASO4 CU \& "FE(OH)3" FRACS=111101110111111 \& $111111111100011110111 \%$ 11

FRAC STREAM $=$ PLYSTBIN SUBSTREAM $=$ CISOLID COMPS=C-S SIO2-S \& AL2O3-S CEMENT FRACS=1 1111

\section{BLOCK DRYER HEATER \\ PARAM TEMP=200 PRES $=1$ \\ BLOCK HETRHEPA HEATER PARAM TEMP=300 $\mathrm{PRES}=1$ \\ BLOCK SOLDRYER HEATER PARAM TEMP $=200$ PRES $=1$ \\ BLOCK CONCENT8 FLASH2 PARAM TEMP=150 PRES $=1$ \\ BLOCK FLASH2 FLASH2 PARAM TEMP $=100$ PRES $=1$}

DESCRIPTION "This models the dryer for the polymer stab. sys."

DESCRIPTION "heater to raise temperature for HEPA filters"

;Data obtained from "Development Studies for a Novel Wet Oxidation Process, ;Topical Report, " Patrick M. Dhooge and Lena B. Hakim, Delphi Research, Inc. ; January 1994, DE-AC21-92MC27109

;Operating conditions: page 63 Detox solution composition: page 2

;Metal solubility: page xvii, 18

;Reaction stoichiometry: page xiv

Excess oxygen: page 58

;Extent of reaction: page xvi ;.

;Temperature and pressure also reported in "Descriptions of Recom ..", p. 2-12

;MK flowsheet shows $423 \mathrm{~K}$ and $20 \mathrm{psi}(+15=35$ psia $)$

BLOCK CWO-1 RSTOIC

DESCRIPTION "wet oxidation reactor"

PARAM TEMP $=423<\mathrm{K}>$ PRES $=35<\mathrm{PSI}>$

STOIC 1 CISOLID C-S -1 / MIXED O2 -1 / CO2 1

STOIC 2 MIXED H2 -2/O2 -1/ $\mathrm{H} 2 \mathrm{O} 2$

STOIC 3 MIXED CL2 -1/ H2 -1/ HCL 2

STOIC 4 MIXED S - $1 / \mathrm{H} 2$ - $1 / \mathrm{O} 2$-2 / H2SO4 1

STOIC 5 MIXED CH4 -1/O2 -2 / CO2 1/ $\mathrm{H}_{2} \mathrm{O} 2$

CONV 1 CISOLID C-S 0.99

CONV 2 MIXED H2 0.99 
CONV 3 MIXED CL2 0.99

CONV 4 MIXED S 0.99

CONV 5 MIXED CH4 0.99

;Data obtained from "Development Studies for a Novel Wet Oxidation Process, ;Topical Report," Patrick M. Dhooge and Lena B. Hakim, Delphi Research, Inc. ; January 1994, DE-AC21-92MC27109

;Operating conditions: page 63

;Detox solution composition: page 2

;Metal solubility: page xvii, 18

;Reaction stoichiometry: page xiv

;Excess oxygen: page 58

;Extent of reaction: page xvi

$;.$

;Temperature and pressure also reported in "Description of Recom.." , p. 2-12

;MK flowsheet shows $423 \mathrm{~K}$ and $20 \mathrm{psi} \mathrm{(+15=35} \mathrm{psia)}$

BLOCK CWO-2 RSTOIC

DESCRIPTION "Wet oxidation reactor"

PARAM TEMP $=423$. $<\mathrm{K}>$ PRES $=35<$ PSI $>$

STOIC 1 CISOLID C-S - $1 /$ MIXED O2 -1/CO2 1

STOIC 2 MIXED H2 -1 / O2 - $0.5 / \mathrm{H} 2 \mathrm{O} 1$

STOIC 3 MIXXED CL2 $-0.5 / \mathrm{H} 2-0.5 / \mathrm{HCL} 1$

STOIC 4 MIXED S - 1 / H2 -1 / O2 -2 / H2SO4 I

CONV 1 CISOLID C-S 0.99

CONV 2 MIXED H2 0.99

CONV 3 MTXED CL2 0.99

CONV 4 MIXED S 0.99

;Data obtained from "Description of Recommended Non-Thermal.." pp. 6-43 to 6-45

;Although room temperature operation is listed on p. $643,1000 \mathrm{C}$ is listed

;on page $6-45$, and the higher temperature is more believable

$; D R E$ of 0.999 listed for TCE and $0.90-0.95$ for PCE $\rightarrow$ use 0.95 for all waste

;assume $1 \mathrm{~atm}$ pressure

BLOCK GPCR RSTOIC

DESCRIPTION "Gas Phase Corona Reactor, see comments"

PARAM PRES $=1$ DUTY $=20000$ SERIES=YES

STOIC 1 MIXED H2 -2/O2-1/ $\mathrm{H} 2 \mathrm{O} 2$

STOIC 2 CISOLD C-S -1 / MIXED O2 -1 / CO2 1

STOIC 3 MIXED H2 - $1 /$ CL2 -1 / HCL 2

STOIC 4 MIXED CO $-1 / \mathrm{O} 2-0.5 / \mathrm{CO} 21$

STOIC 5 MTXED CH4 -1 / O2 -2/ CO2 1/ $\mathrm{H} 2 \mathrm{O} 2$

CONV 1 MIXED H2 0.95

CONV 2 CISOLID C-S 0.95 .

CONV 3 MIXED CL2 0.95

CONV 4 MIXED CO 0.95

CONV 5 MIXED CH4 0.95

BLOCK NEUTRAI 1 RSTOIC

DESCRIPTION "neutralizes excess $\mathrm{HCl}$ "

PARAM TEMP=100 PRES $=1$

STOIC 1 MIXED NAOH - 1 / HCL - 1 / NACL $1 /$ H2O 1

STOIC 2 MDXED H2SO4 - 1 / NAOH - 2 / NA2SO4 1 / $\mathrm{H}_{2} \mathrm{O} 2$

STOIC 3 MIXED CL2 - 1 / NAOH - 2 / NACL 2 / H2O 1 / \&

020.5

STOIC 4 MIXED FECL3 -1 / NAOH -3 / "FE(OH)3" 1 / NACL \& 3 
CONV 1 MIXED HCL 0.99

CONV 2 MIXED $\mathrm{H} 2 \mathrm{SO} 40.99$

CONV 3 MIXED CL2 0.99

CONV 4 MIXED FECL 30.99

BLOCK NEUTRAL2 RSTOIC

DESCRIPTION "neutralizes excess HCl"

PARAM TEMP=100 PRES $=1$

STOIC 1 MIXED NAOH - 1 / HCL - $1 /$ NACL $1 /$ H2O 1

STOIC 2 MIXED H2SO4 -1/ NAOH - $/$ NA2SO4 $1 /$ H2O 2

STOIC 3 MIXED CL2 - $1 /$ NAOH -2 / NACL $2 / \mathrm{H} 2 \mathrm{O} 1 /$ \&

$\mathrm{O} 20.5$

STOIC 4 MIXED FECL3 - 1 / NAOH -3 / "FE(OH)3" 1 / NACL \& 3

CONV 1 MIXED HCL 0.99

CONV 2 MIXED H2SO4 0.99

CONV 3 MIXED CL2 0.99

CONV 4 MIXED FECL 30.99

;scrubbing efficiency of 0.99 assumed for now

BLOCK NEUTRAL3 RSTOIC

DESCRIPTION "neutralizes $\mathrm{HCl}$ in offgas stream"

PARAM TEMP $=100$ PRES $=1$

STOIC 1 MIXED HCL - 1 / NAOH - 1 / NACL $1 /$ H2O 1

STOIC 2 MIXED CL2 - 1 / NAOH -2 / NACL 2 / H2O 1 / \& 020.5

STOIC 3 MLXED H2SO4 - 1 / NAOH -2 / NA2SO4 $1 /$ H2O 2

STOIC 4 MIXED SO2 - $1 / \mathrm{NAOH}-2 / \mathrm{O} 2-0.5$ / NA2SO4 1 \&

/ $\mathrm{H} 20 \mathrm{O}$

CONV 1 MIXED HCL 0.99

CONV 2 MIXED CL2 0.99

CONV 3 MIXED H2SO4 0.99

CONV 4 MIXED SO2 0.99

BLOCK OH-GEN RSTOIC

PARAM IEMP $=100$ PRES $=1$

STOIC 1 MIXED H2O2 -1/ OH-RAD 2

STOIC 2 MIXED O3 -1 / H2O -3 / OH-RAD 6

CONV 1 MIXED H2O2 0.99

CONV 2 MIXED 030.99

BLOCK OZ-DECOM RSTOIC

DESCRIPTION "ozone decomposer"

PARAM TEMP $=100$ PRES $=1$

STOIC 1 MIXED O3 -2 / O2 3

STOIC 2 MIXED HCL - $1 /$ NAOH - $1 /$ NACL $1 /$ H2O 1

STOIC 3 MIXED H2SO4 - $/$ NAOH -2/ NA2SO4 $1 /$ H2O 2

STOIC 4 MIXED CL2 - 1 / NAOH - $/$ NACL 2 / H2O 1 / \&

$\mathrm{O} 20.5$

STOIC 5 MIXED SO2 -1 / NAOH -2 / O2 -0.5 / NA2SO4 1 \& / $\mathrm{H} 2 \mathrm{O} 1$

CONV 1 MIXED 030.999

CONV 2 MIXED HCL 0.99

CONV 3 MIXED H2SO4 0.99

CONV 4 MIXED CL2 0.99

CONV 5 MIXED SO2 0.99 


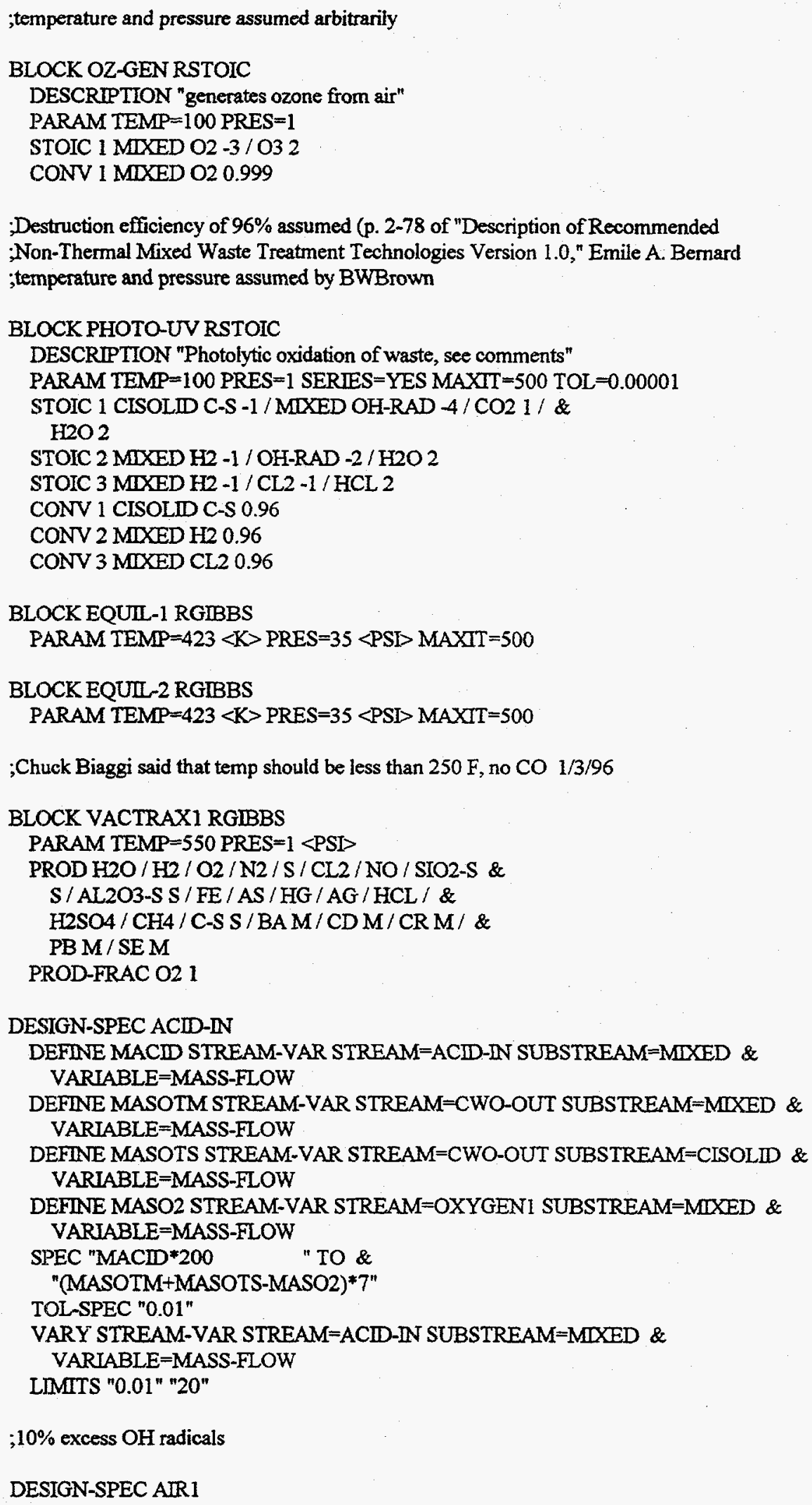

;Destruction efficiency of $96 \%$ assumed (p. 2-78 of "Description of Recommended ;Non-Thermal Mixed Waste Treatment Technologies Version 1.0, " Emile A. Bernard ;temperature and pressure assumed by BWBrown 


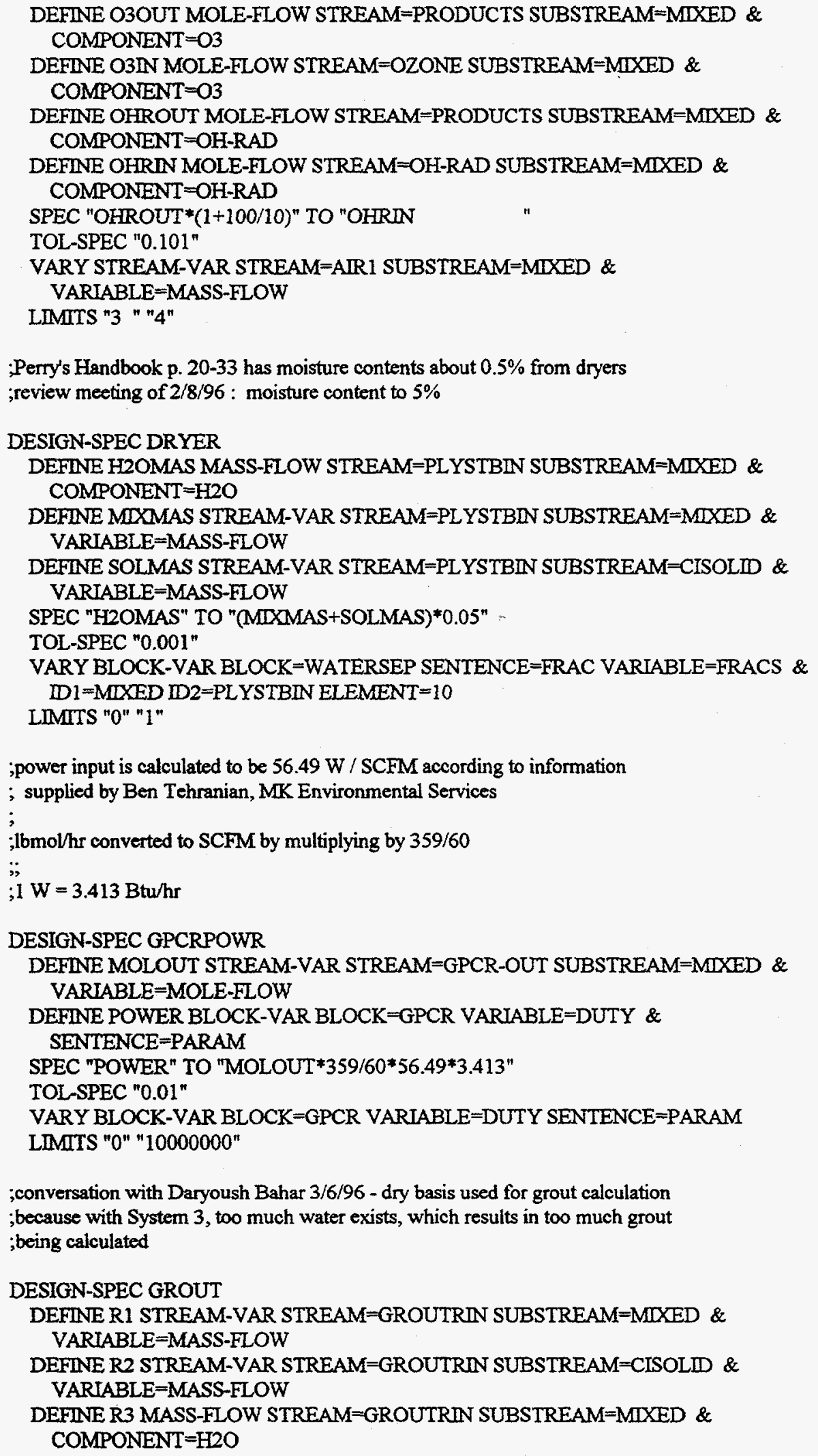




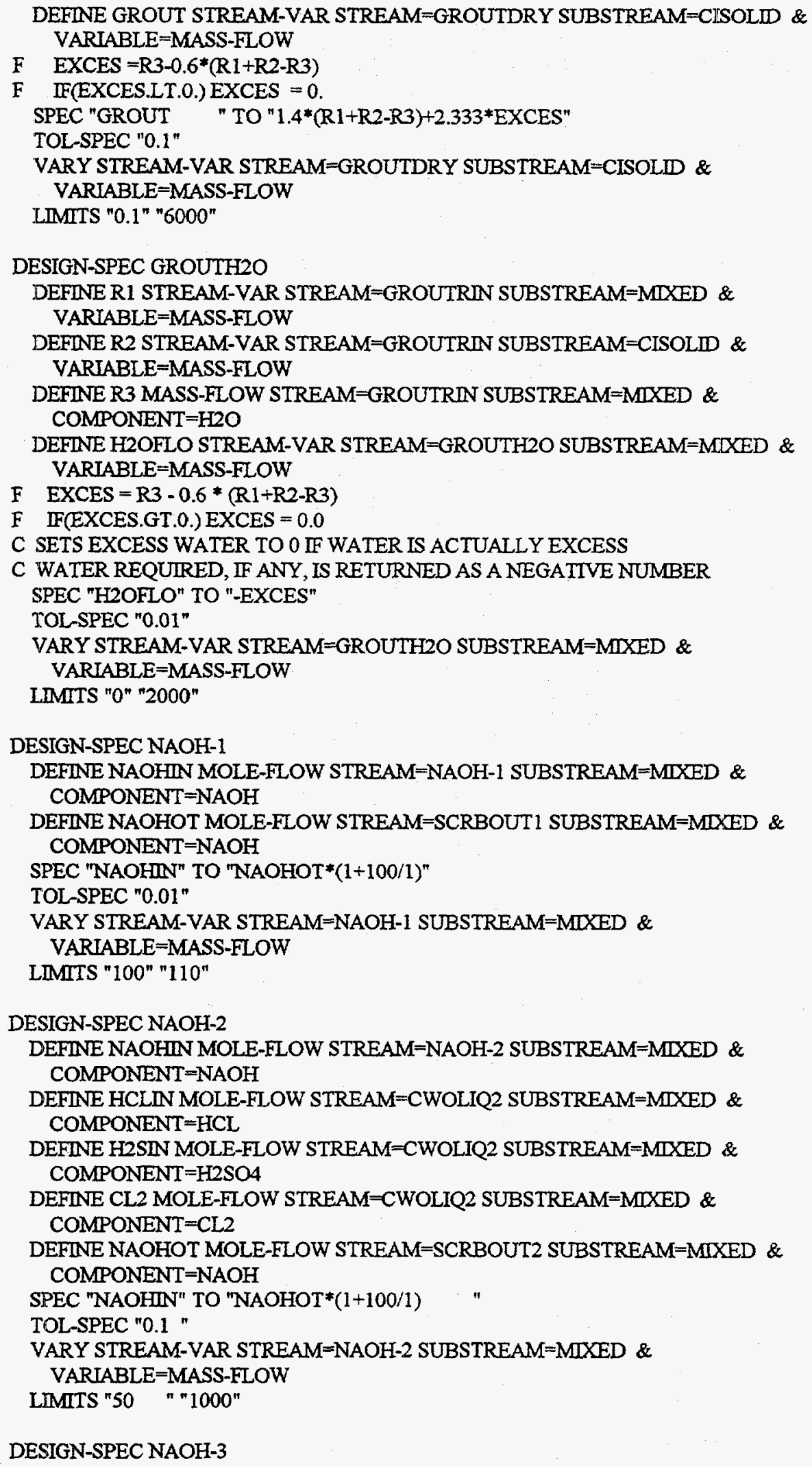

DESIGN-SPEC NAOH-3 


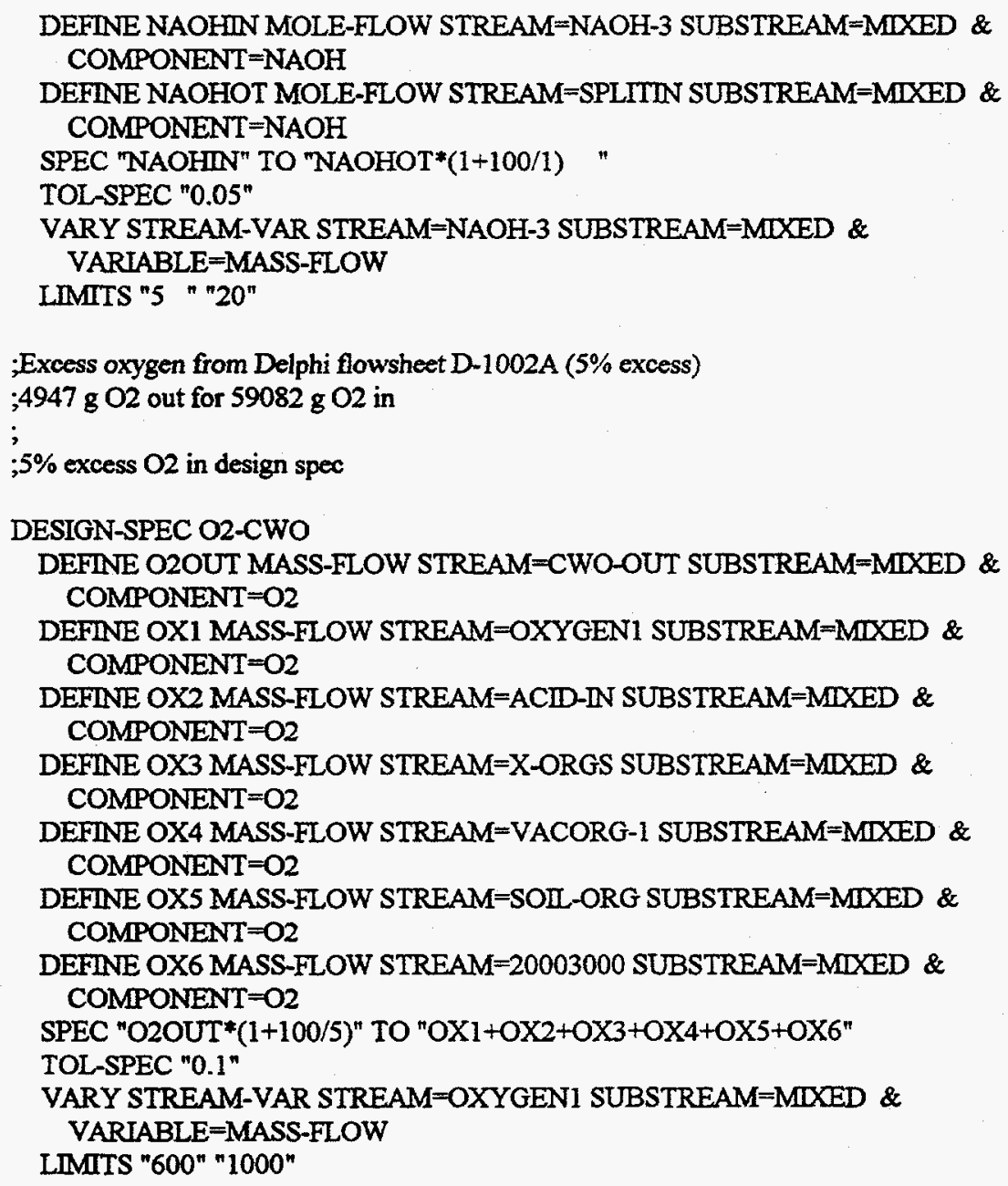


VARIABLE = MASS-FLOW

DEFINE SOLDIN STREAM-VAR STREAM=PLYSTBIN SUBSTREAM=CISOLID \& VARIABLE=MASS-FLOW

DEFINE POLYIN STREAM-VAR STREAM=POLYETHL SUBSTREAM=MTXED \& VARIABLE=MASS-FLOW

SPEC "POLYIN" TO "(WASTIN+SOLDIN)"

TOL-SPEC "0.1"

VARY STREAM-VAR STREAM=POLYETHL SUBSTREAM=MIXED \& VARIABLE=MASS-FLOW

LMITS "0.1" "5000"

$; 20 \%$ moisture content set at review meeting $2 / 8 / 96$

DESIGN-SPEC SALTSOLN

DEFINE SALTFL MASS-FLOW STREAM=SALTSOLN SUBSTREAM=MIXED \& COMPONENT $=$ NACL

DEFINE H2OFLO MASS-FLOW STREAM=SALTSOLN SUBSTREAM=MIXED \& COMPONENT $=\mathrm{H} 2 \mathrm{O}$

DEFINE NAOHFL MASS-FLOW STREAM=SALTSOLN SUBSTREAM=MIXED \& COMPONENT $=\mathrm{NAOH}$

SPEC "H2OFLO*4" TO "SALTFL+NAOHFL"

TOL-SPEC "0.01"

VARY BLOCK-VAR BLOCK=BLOWDOWN SENTENCE $=F R A C$ VARIABLE $=F R A C S \&$ $\mathrm{ID} 1=\mathrm{MIXED}$ ID2 $=$ FLASH-N ELEMENT $=11$

LIMITS "0.001" "1"

DESIGN-SPEC XX-NAOH

DEFINE NAOHIN MASS-FLOW STREAM=PRODUCTS SUBSTREAM=MIXIED \& COMPONENT $=$ NAOH

DEFINE NAOHOT MASS-FLOW STREAM=AQ-OUT SUBSTREAM=MIXED \& COMPONENT $=\mathrm{NAOH}$

SPEC "NAOHIN" TO "NAOHOT* $(1+100 / 1)$ "

TOL-SPEC "0.10"

VARY STREAM-VAR STREAM $=\mathrm{XX}-\mathrm{NAOH}$ SUBSTREAM=MOXED \& VARIABLE=MASS-FLOW

LIMITS " $17 "$ "18.5"

FORTRAN ADDITIVE

DEFINE HGOUT MASS-FLOW STREAM=HG-OUT SUBSTREAM=MTXED \& COMPONENT $=\mathrm{HG}$

DEFINE COPPIN MASS-FLOW STREAM=ADDITTVE SUBSTREAM=MIXED \& COMPONENT $=\mathrm{CU}$

F $\quad$ COPPIN $=0.7 *$ HGOUT

READ-VARS HGOUT

WRITE-VARS COPPIN

FORTRAN $\mathrm{H} 2 \mathrm{O} 2-\mathrm{NN} 2$

DEFINE O3IN MOLE-FLOW STREAM=PHOTO-IN SUBSTREAM=MIXED \& COMPONENT $=\mathrm{O} 3$

DEFINE H2O2IN STREAM-VAR STREAM=H2O2-IN2 SUBSTREAM=MIXED \& VARIABLE=MOLE-FLOW

F $\mathrm{H} 2 \mathrm{O} 2 \mathrm{NN}=\mathrm{O} 3 \mathrm{~N}$

READ-VARS O3N

WRITE-VARS H2O2N

; constant is obtained by taking $1200 \mathrm{ft} 3 / 1200 \mathrm{lb}$ and mult. by $0.0727 \mathrm{lb} / \mathrm{ft} 3$

FORTRAN N2SWEEP

B-106 
DEFINE N2SWP1 STREAM-VAR STREAM=N2SWEEP1 SUBSTREAM=MIXED \& VARIABLE=MASS-FLOW

DEFINE MS3000 STREAM-VAR STREAM $=3000$ SUBSTREAM $=$ CISOLID \& VARIABLE=MASS-FLOW

DEFINE MM3000 STREAM-VAR STREAM $=3000$ SUBSTREAM=MIXED \& VARIABLE $=$ MASS-FLOW

F $\quad \mathrm{N} 2 \mathrm{SWP1}=0.0793 *(\mathrm{MS} 3000+\mathrm{MM} 3000)$

READ-VARS MS $3000 \mathrm{MM} 3000$

WRTTE-VARS N2SWP1

\section{CONV-OPTIONS}

PARAM TEAR-METHOD $=$ BROYDEN TOL $=0.00011$

BROYDEN MAXIT $=200$

TEAR

TEAR PRODUCTS 0.1

STREAM-REPOR MOLEFLOW MASSFLOW

;

;

;

;

; 